\title{
Electroacoustical simulation of listening room acoustics for project ARCHIMEDES
}

\author{
Bech, Søren
}

Published in:

Acoustical Society of America. Journal

Link to article, DOI:

$10.1121 / 1.2027447$

Publication date:

1989

Document Version

Publisher's PDF, also known as Version of record

Link back to DTU Orbit

Citation (APA):

Bech, S. (1989). Electroacoustical simulation of listening room acoustics for project ARCHIMEDES. Acoustical Society of America. Journal, 86(S1), S2-S2. https://doi.org/10.1121/1.2027447

\section{General rights}

Copyright and moral rights for the publications made accessible in the public portal are retained by the authors and/or other copyright owners and it is a condition of accessing publications that users recognise and abide by the legal requirements associated with these rights.

- Users may download and print one copy of any publication from the public portal for the purpose of private study or research.

- You may not further distribute the material or use it for any profit-making activity or commercial gain

- You may freely distribute the URL identifying the publication in the public portal

If you believe that this document breaches copyright please contact us providing details, and we will remove access to the work immediately and investigate your claim. 


\section{PROGRAM OF}

The 118th Meeting of the Acoustical Society of America

Adam's Mark Hotel • St. Louis, Missouri • 27 November-1 December 1989

MONDAY EVENING, 27 NOVEMBER 1989

ST. LOUIS BALLROOM D, 7:00 TO 9:00 P.M.

\section{Tutorial on Architectural Acoustics}

Mauro Pierucci, Chairman

Department of Aerospace and Engineering Mechanics, San Diego State University. San Diego,

California 92182

TU1. Architectural acoustics: The forgotten dimension. Ewart A. Wetherill (Wilson, Ihrig, and Associates, Inc., 5776 Broadway, Oakland, CA 94618)

The basic considerations of architeclural acoustics-isolation from unwanted noise and vibration, control of mechanical system noise, and room acoustics design-are all clearly exemplified in Sabine's design for Boston Symphony Hall. Opened in 1900, this hall is one of the outstanding successes in musical acoustics. Yet, as we approach the hundredth anniversary of Sabine's first experiments, acoustical characteristics remain one of the least considered aspects of building design. This is due, in part, to the difficulty of visualizing the acoustical outcome of design decisions, complicated by individual judgment as to what constitutes good acoustics. However, the lack of a comprehensive teaching program remains the dominant problem. Significant advances over the past 2 or 3 decades in measurement and evaluation have refined the ability to design predictability and to demonstrate acoustical concerns to others. New techniques such as sound intensity measurements, new descriptors for room acoustics phenomena, and the refinement of recording, analysis, and amplification techniques provide fresh insights into the behavior of sound in air and other media. These topics are reviewed with particular emphasis on the need for a comparable advance in translation of acoustic principles into building technologies. 


\title{
Session A. Architectural Acoustics I: Electronic Room Simulation for Production and Reproduction
}

\author{
Elizabeth A. Cohen, Cochairman \\ Charles M. Salter Associates, Inc., 130 Sutter Street, San Francisco, California 94101 \\ J. Christopher Jaffe, Cochairman \\ Jaffe Acoustics, Inc., 114 A Washington Street, Norwalk, Connecticut 06854 \\ Chairman's Introduction-8:00 \\ Theodore John Schultz-A Tribute \\ Special Tribute-8:05 \\ Presented by: Ewart A. Wetherill (Wilson, Ihrig, and Associates, 5776 Broadway, Oakland, California 94618)
}

\section{Invited Papers}

\section{8:15}

A1. Electroacoustical simulation of listening room acoustics for project ARCHIMEDES. Søren Bech (The Acoustics Laboratory, Technical University of Denmark, Building 352, DK-2800 Lyngby, Denmark)

ARCHIMEDES is a psychoacoustics research project, funded under the European EUREKA scheme. Three partners share the work involved: The Acoustics Laboratory of The Technical University of Denmark; Bang and Olufsen of Denmark; and KEF Electronics of England. Its primary object is to quantify the influence of listening room acoustics on the timbre of reproduced sound. For simulation of the acoustics of a standard listening room, an electroacoustic setup has been built in an anechoic chamber. The setup is based on a computer model of the listening room, and it consists of a number of loudspeakers positioned on an imaginary sphere surrounding the position of the test subject. The setup has been designed for the highest degree of flexibility. This includes the possibility of simulation of directivity characteristics of normal domestic loudspeakers and absorption coefficients of the surfaces of the listening room. This paper is a presentation of the system, with special emphasis on the psychoacoustical background of the design. This will include a discussion of choice of experimental procedure, test stimuli, and test subjects as well as purpose built loudspeakers and the DSP system.

A2. The psychoacoustics of loudspeaker sound reproduction-Past achievernents and present problems. Floyd E. Toole (Division of Physics, National Research Council, Ottawa, Ontario K1A 0R6, Canada)

The design of loudspeakers is gradually being put on a scientific basis. Art and intuition have given way to engineering guidelines as the relationships between perceptions and technical measurements have been elucidated. Indeed, within limited circumstances, loudspeakers can be designed to meet specific engineering design objectives, with considerable confidence in how they will be subjectively evaluated. In practice, however, all of the relevant conditions are not controlled, and several factors conspire to preclude universal satisfaction among listeners. Most of the uncertainty appears to be in the interactions between loudspeakers, rooms, listeners, and program material. This paper reviews the present state of knowledge, and outlines the areas most in need of further work. It is clear that additional psychoacoustical data and suitable technical innovations can alleviate some of the remaining problems. Others, though, may be better treated by standardization.

A3. RODS-An advanced approach to electronic reverberation enhancement. Peter W. Barnett (AMS Acoustics, Ltd., 52 Chase Side Southgate, London N14 5PA, United Kingdom)

This paper will briefly review the recent history of reverberation enhancement and then examine the processes involved in the development of the RODS (Reverberation-on-Demand System) concept. The theory and implementation of the RODS concept will be explained, followed by examples and results of recent installations. 
A4. Equalization using voice and music as the source. John Meyer (Meyer Sound Laboratories, 2832 San Pablo Avenue, Berkeley, CA 94702)

A source-independent technique to measure accurately the amplitude and phase response of sound systems in concert halls is discussed. Measurements may be made during live performances or events, using music or voice as the test signals. Correlation is shown between the impulse response and the results obtained using music signals. An equalizer that corrects for many room resonances in both amplitude and phase simultaneously has been developed. The effect of this equalizer on concert systems in an existing venue is shown.

\title{
10:15
}

A5. A spatial sound processor for headphone and loudspeaker reproduction. William L. Martens, Gary S. Kendall, and Martin D. Wilde (SoundSphere, Inc., 1030 Davis Street, Evanston, IL 60201)

A spatial sound processor for stereo headphone and loudspeaker reproduction is described that can position sound elements within a three-dimensional reverberant space surrounding the listener. Spatial motion of sound sources in three dimensions is created by dynamic filtering based on head-related transfer functions. Additional filters and delay lines capture air absorption and Doppler shifting as the propagation time is manipulated for both direct and indirect sound. The spatiotemporal distribution of early reflections is captured for a given source/listener orientation: The gain, delay, and directional filtering of simulated reflections are responsive to changes in the specified position and orientation of the sound source and the listener's head in the simulated environment. The spatial processor can be used for headphone reproduction using a head-tracking device, and can also be used in more typical reproduction settings such as living rooms with stereo loudspeakers. In the latter case, additional processing is employed to stabilize the stereo image and produce a spatially diffuse reverberant surround effect over a wide range of listening positions.

\author{
10:45-11:00
}

Break

\section{Contributed Papers}

11:00

A6. Binaural simulation technique for scale modeling. N. Xiang and J. Blauert (Lehrstuhl für Allgemeine Elektrotechnik und Akustik, Ruhr Universität, D-4630 Bochum 1, Federal Republic of Germany)

Room simulation for the purpose of predicting acoustic behavior and quality has recently become a popular topic in room acoustics. At the Ruhr University, the experience of the binaural human listener in a room simulation using both physical and computer modeling has been authentically recreated. This paper reports on the latest stage of the present work in binaural room simulation using a scaled down physical model, where a sensitive miniature dummy head with accurately scaled pinnae (scale factor 1:10) serves as a receiver to pick up the model sound field. A versatile PC-based system measures the binaural impulse responses in model space according to the $m$-sequence transform and also carries out the convolution of the room-impulse responses with anechoic speech or music signals. An especially wide broadband ultrasonic transmitter system is necessary to provide the desired complex stimulation of the sound field. After the necessary signal processing, the resulting signals can be listened to binaurally via headphones. [Work supported in part by Deutsche Forschungsgemeinschaft.]
11:15

A7. On the model order for the identification of acoustical systems. Nobuo Koizumi (NTT Human Interface Laboratories, Tokyo 180, Japan) and Richard H. Lyon (Department of Mechanical Engineering, MIT, Cambridge, MA 02139)

The pole/zero model and the finite impulse response (FIR) model are used as system models for the identification of unknown acoustical systems. The size of the model is particularly important in discrete-time implementation as it determines the convergence rate of adaptation and capacity of real-time processing. The order of the pole/zero model is related to modal distribution of systems while the order of the FIR model depends on its damping factor. Effective orders of both models are estimated from the statistical properties of acoustical systems. In a threedimensional enclosure, its volume and reverberation time are used for estimation. It is shown that, when modal density of the system is low, such as in a small enclosure, and frequency range is narrow, pole/zero modeling can greatly reduce the model order. [Work supported by NTT Human Interface Laboratories, Tokyo 180, Japan.] 


\title{
Session B. Physical Acoustics I: Scattering, Propagation, Diffraction, and Reflection
}

\author{
Julian D. Maynard, Chairman \\ Department of Physics, Pennsylvania State University, University Park, Pennsylvania 16802
}

\author{
Contributed Papers
}

\begin{abstract}
8:00
B1. Consequences of the acoustic bipolar couplings to large aspect ratio scatterers. Roger H. Hackman (Physical Acoustics Branch, Naval Coastal Systems Center, Panama City, FL 32407)

It has been suggested that a plane wave axially incident on a large aspect ratio scatterer couples to both the insonified end of the scatterer and to the end in the geometric shadow, at low $k D / 2$ ( $D$ is either the minor axis of a spheroid or the diameter of a cylinder) [Williams et al., J. Acoust. Soc. Am. 85, 2372-2377 (1989) ]. This effect is concretely established, and its experimental consequences are discussed in some detail. A novel closed-form expression is derived for the axisymmetric, elastic response of a large aspect ratio target that directly incorporates the bipolar coupling of the acoustic and elastic fields. The form of the final expression is, in some respects, similar to that obtained from the generalized geometric theory of diffraction in the high-frequency limit for elastic spheres and cylinders, although the physical assumptions are quite different. Simple, approximate estimates of the parameters involved in this expression are obtained and compared with a $T$-matrix calculation.
\end{abstract}

\section{8:15}

B2. The acoustic scattering by a submerged, elastic spherical shell: The transition from thin to thick shells. Gary S. Sammelmann and Roger H. Hackman (Physical Acoustics Branch, Naval Coastal Systems Center, Panama City, FL 32407)

A fundamentally oriented analysis of the pole structure of the acoustic scattering matrix in the low-to high-frequency region $(0 \leqslant k a \leqslant 1000)$ as a function of mode number, density, and sound speed for several shell thicknesses has been previously presented [J. Acoust. Soc. Am. Suppl. 183, S94 (1988); Suppl. 1 84, S185 (1988); Suppl. 185 , S95 (1989)]. The three most interesting results of this analysis were: (1) Due to fluid loading, the vacuum antisymmetric Lamb mode $a_{0}$ and the rigid Franz modes "switch tails" to produce a subsonic $a_{0-}$ mode that resembles the vacuum antisymmetric Lamb mode at low $k a$ and an $a_{0+}$ mode that resembles $a_{0}$ at high $k a$; (2) the mid-frequency enhancement of a thin shell is associated with this bifurcation of the antisymmetric Lamb wave [J. Acoust. Soc. Am. 85, 114-124 (1989) ]; and (3) the existence of strong thickness resonances associated with the existence of regions of negative group velocity for the third antisymmetric Lamb wave on a thin spherical shell. In this presentation, the trajectories of the poles of the acoustic $S$ matrix as a function of the thickness of the shell are followed. A unified picture of the resonance structure of a spherical shell as a function of shell thickness is presented, and the importance of tail switching of the elastic and diffractive degrees of freedom in the transition from a thick to a thin shell is illustrated.

\section{8:30}

B3. Acoustic interactions with buried objects. Raymond Lim and Roger H. Hackman (Physical Acoustics Branch, Naval Coastal Systems Center, Panama City, FL 32407)

The theoretical description of the scattering from objects near medium boundaries has been the subject of a number of recent articles [e.g., G.
Kristensson and S. Ström, J. Acoust. Soc. Am. 64, 917-936 (1978); G. S. Sammelmann and R. H. Hackman, J. Acoust. Soc. Am. 82, 324-336 (1987) ]. These articles consider scatterers in nonattenuating, liquid media. When objects buried in sediment are considered, however, sediment attenuation introduced fundamentally new features into the description of the scattering process. The interaction of an incident plane wave with the sediment-seawater interface distorts the phase-amplitude relation of the wave incident on a scatterer in the sediment. The description of the scattering of these inhomogeneous waves requires the introduction of new, "suitably modified" basis states for the solution of the scattering problem. The modification of the $T$-matrix and waveguide theories developed at the Naval Coastal Systems Center is discussed and a numerical study of the scattering by a thin spherical shell buried in an attenuating liquid sediment is presented.

\section{$8: 45$}

B4. High-frequency acoustic scattering from a doubly periodic ellipsoidal surface: Neumann boundary conditions. Garner $C$. Bishop and Judy Smith (Naval Underwater Systems Center, Newport, RI 02840)

An exact solution is obtained for the scatter of an acoustic plane wave from an infinite surface constructed from a doubly periodic array of infinite and parallel elliptical semicylinders. Neumann boundary conditions are imposed and the Helmholtz-Kirchhoff integral used to calculate the scattered pressure field. Fredholm integral equations of the first and second kind are used to calculate the surface field. Numerical calculations are performed to determine the effects of geometric parameters on the scattered pressure field and the dependence of the surface field on the type of Fredholm integral equation used. Results are also compared with those for scattering from a sinusoidal surface.

\section{9:00}

B5. Contributions to the form function for elastic spheres based on a product expansion of the $S$ function: Numerical tests. Philip $L$. Marston (Department of Physics, Washington State University, Pullman, WA 99164)

Associated with the scattering phase shift $\delta_{n}$ of the $n$th partial wave for a sphere of radius $a$ is the function $S_{n}(x)=\exp \left[2 i \delta_{n}\right]$, where $x=k a$. A theorem from classical scattering theory leads to a product expansion of $S_{n}$, which allows for multiple resonances [P. L. Marston, J. Acoust. Soc. Am. Suppl. 184, S185 (1988) ]. This $S_{n}$ remains manifestly unitary even for multiple resonances / and clarifies implicit assumptions of RST. The elastic contributions $f_{n}^{e}$ to the form function depend on complex $k a$ pole locations $x_{n l} \equiv X_{n t}-i\left(\Gamma_{n l} / 2\right)$ and $-x_{n l}^{*}$, where $X_{n l}>0, \Gamma_{n l}>0$, and * denotes complex conjugation. In the case of only two resonances $(l=1,2)$, it may be shown that $f_{n}^{c} \approx f_{n 1}+f_{n 2}+f_{n}^{(\text {inl })}+f_{n}^{L}$. The $f_{n \prime}$ have a Breit-Wigner form $f_{n t}=\exp \left(2 i \xi_{n}\right)[(2 n+1) / x](-1)^{n} \Gamma_{n \prime} /\left[X_{n t}\right.$ $\left.-x-(i / 2) \Gamma_{n l}\right]$ and $\xi_{n}$ is the phase shift associated with the background factor of $S_{n}$. The term $f_{n}^{L}$ is a term associated with the pole at $-x_{n \prime}^{*}$ in the left half of the complex $k a$ plane while $f_{n}^{(\text {int) }}$ is an interaction term. The effect of $f_{n}^{L}$ is small if $\Gamma_{n l} \ll X_{n t}$. This approximation of $f_{n}^{e}$ is confirmed by numerical comparison with the $f_{n}^{c}$ based directly on the 
product expansion. The comparison also shows that omission of the term $f_{n}^{(\mathrm{ini})}$ can introduce substantial errors for $x$ between $X_{n 1}$ and $X_{n 2}$. [ Work supported by ONR.]

\section{9:15}

B6. Lips caustics in light backscattered from an acoustically levitated spheroidal water drop. Harry J. Simpson and Philip L. Marston (Department of Physics, Washington State University, Pullman, WA 99164-2814)

Cusp diffraction catastrophes that open up roughly transverse to the propagation direction are known to exist for both light and sound [P. L. Marston, J. Acoust. Soc. Am. 81, 226-232 (1987) ]. They may be produced by reflecting high-frequency sound from curved surfaces. In the present research, a closely related caustic in which the cusp curves join to form a pair of lips is studied. This caustic was predicted to exist [ J. F. Nye, Nature 312, 531-532 (1984)] in light backscattered from horizontally illuminated oblate drops of water, provided the axis ratio $q=D / H$ was within certain ranges. The associated rays are diffracted at the drop's surface and have only one internal reflection. As $q$ is increased above the critical value $q_{4} \approx 1.31$ associated with a hyperbolic umbilic focal section, the cusp points at the ends of the lips caustic were predicted to merge in the backward direction at a "lips event" when $q=q_{L 1} \approx 1.42$. For $q_{L_{1}}<q<q_{L_{2}} \approx 1.58$, no caustics for this class of ray are expected while a second lips event occurs at $q_{L_{2}}$. Observations of farfield scattering from levitated drops support Nye's analysis and illustrate a mechanism for producing lips caustics. The observed backscattering is weak for $q$ between $q_{L 1}$ and $q_{L 2}$. [Work supported by ONR.]

\section{9:30}

B7. Quasiperiod of variations in the backscattering and total cross sections of spherical shells. Steven G. Kargl and Philip L. Marston (Department of Physics, Washington State University, Pullman, WA 99164-2814)

Quantitative ray methods for leaky surface elastic waves are applied [P. L. Marston, J. Acoust. Soc. Am. 83, 25-37 (1988)] to approximate the quasiperiod of structures in backscattering and total scattering cross sections. The relevant amplitudes in this model contain a Fabry-Perot resonance denominator, which for spheres is $[1+\exp (-2 \pi \beta$, $\left.\left.+i 2 \pi x c / c_{l}\right)\right]$ for the $l$ th class of SEW; $x=k a=\omega a / c$ and $c_{l}(x)$ and $\beta_{l}(x)$ are the SEW phase velocity and radiation damping coefficient. The increment in $x$ between resonances for a given $l$ follows from the condition $\Delta\left(x c / c_{1}\right)=1$. From the Taylor expansion $\left[c_{l}(x+\Delta x)\right]^{-1}$ $\approx c_{1}(x)^{-1}+\Delta x d\left(c_{1}^{-1}\right) / d x$, the approximate resonance spacing $\Delta x \approx c_{g l} / c$ for weakly dispersive SEW having a group velocity $c_{g}(x)$ is obtained. (This approximation has been noted by others.) The quasiperiod of a broad structure in the total scattering cross section is also approximated as $\Delta x_{l} \approx 2 \pi\left[2 \cos \theta_{l}-\left(c / c_{l}\right)\left(\pi-2 \theta_{l}\right)\right]^{-1}, \sin \theta_{l}=c / c_{l}$, when dispersion is neglected. This follows from a condition on the phase increment of the forward amplitude. Both types of variations due to Lamb wave contributions can be seen in exact and ray-synthesis calculations for a spherical shell. [Work supported by ONR.]

\section{9:45}

B8. New rigorous and ray-acoustic traveling wave formulations for source-excited thin elastic spherical shells immersed in fluids. L. B. Felsen and J. M. Ho (Department of Electrical Engineering and Computer Science, Polytechnic University, Farmingdale, NY 11735)

A continuous Legendré transform spanning the polar angle $(\theta)$ domain $-\infty<\theta<\infty$ is employed to derive a rigorous new integral repre- sentation for source-excited-time-harmonic pressure fields in the presence of a thin elastic spherical shell immersed in different interior and exterior fluids. The new formulation identifies directly the traveling waves with their multiple encirclements of the shell by extending the $\theta$ domain from its conventional $0 \leqslant \theta \leqslant \pi$ range with periodicity constraints into the unbounded (multisheeted) domain without these constraints. The new formulation also systematizes the treatment of spherical caps and other truncated sections. Periodicity for the closed shell is recovered by summing contributions from an infinite array of image sources located in the "nonphysical" portion $\theta<0$ and $\theta>\pi$ of the angular space. The rigorous solution is obtained by synthesis over a complex spectral continuum, and various alternative representations are derived from it. Special attention is given to rigorous high-frequency asymptotic forms that describe the wave phenomena in terms of incident and geometrically reflected ray fields, and also in terms of surface guided ray fields. The latter are excited by phase matching of the incident ray field to the traveling wave modes in the shell, and they reach the observer by phase matched detachment [see, also, P. L. Marston, J. Acoust. Soc. Am. 83, 25-37 (1988)]. The phase matching applies to directly excited leaky waves as well as to waves that decay initially into the fluid; the latter are excited from an exterior source by evanescent tunneling. [Work supported by the Office of Naval Research and David Taylor Research Center.]

\section{0:00}

B9. The optical illusion of sound. Bradford L. Swartz (Communication Disorders, Central Michigan University, Mt. Pleasant, MI 48859)

The graphic depiction of sound is not a straightforward matter. Stationary representations using lines, waves, or circles leave the notion of movement (either particle or energy) and pressure fluctuations within the wave to the reader's imagination. The common sinuous wave shape evident in most all sources of study originates from Lissajous, and resulted from his transfer of tuning fork motions into a point of reflected light that traversed a screen. Lissajous performed his experiments with finely focused electric lamp light, tuning forks, and mirrors. Similar experiments can be replicated today using laser light and audio speakers. Thus one can more readily gain an appreciation for the familiar wave pattern representing sound, and see by the simple instruments used to create it, how this waveform is not a true depiction of the shape of sound waves in a medium. It is in reality a displacement versus time graph. By using multiple forks or speakers in opposing planes and tuned to various frequencies, the creation of the single- and multiple-ellipse Lissajous patterns that are often produced on an oscilloscope screen can also be accomplished.

\section{0:15}

B10. Acoustic surface shape resonances. Alexei A. Maradudin (Department of Physics, University of California, Irvine, CA 92717)

Acoustic surface shape resonances are vibrational excitations that are localized in the vicinity of an isolated protuberance or indentation on the otherwise planar, stress-free surface of a semi-infinite elastic medium. The protuberance may be fabricated from the same material as the substrate, or from a different material. In general, there is an infinite number of such resonances associated with a given surface perturbation. Their frequencies are discrete because of the loss of translational symmetry caused by the surface perturbation; they are complex because they overlap the range of frequencies allowed the vibrations of the substrate, into which they can decay; and they depend on the shape of the protuberance or indentation, and on the relation of the material properties of the protuberance (mass density, elastic moduli) to those of the substrate. Methods for calculating the frequencies of the acoustic surface shape resonances associated with protuberances or indentations of simple forms are described. Results are presented that suggest that acoustic surface shape resonances can be studied experimentally by the scattering of acoustic waves from the structure supporting them. 
B11. Diffraction of a point source about a disk. J. J. Dlubac, C. W. Jiang, and B. Yim (David Taylor Research Center, Bethesda, MD 200845000 )

Computations of diffraction of an acoustic point source about a hard oidal wavefunctions, is based on the classical analysis [J. J. Bowman, T. B. A. Senior, and P. L. E. Uslenghi, Electromagnetic and Acoustic Scattering by Simple Shapes (North-Holland, Amsterdam, The Netherlands, 1969), Chap. 14]. In the limit as the source goes to infinity, the plane-wave solution is recovered. Computations with the field point on the disk verify the boundary conditions. Parameter studies are presented, and convergence criteria are discussed. and soft disk are presented. The solution, given as a series of oblate spher-

B14. Prediction of bending resonances on elastic spheroids due to oblique incident plane waves from exact beam theory and comparison to $T$-matrix calculations. M. F. Werby (NORDA, Numerical Modeling, Stennis Space Center, MS 39529) and G. C. Gaunaurd (Naval Surface Warfare Center, White Oak Laboratory, Silver Spring, MD 20903)

Exact beam theory predicts the presence of "bending" or flexural resonances from oblique incident plane waves. In an earlier paper, Timoshenko theory, which assumes a constant moment of inertia along the beam, was employed to derive an approximate theory to predict flexural resonances. Good agreement was found (for the lower modes) with the exact $T$-matrix calculations based on exact 3 -D elastodynamics. It is possible to solve the exact beam equation using phase matching techniques to predict "bending" resonance locations. Such calculations are performed for spheroids for aspect ratios from 1.5 to 10 , with extremely good agreement between beam theory and the exact $T$-matrix calculations for all modes.

\section{0:45}

B12. Reflectivity of monolayers of bubblelike scatterers at water/solid interfaces. Ivan Tolstoy (Knockvennie, Castle Douglas DG7 3PA, SW Scotland)

By redistributing the energy flux of an incident plane wave, a monolayer of identical bubblelike scatterers at an interface may, at frequencies close to the monopole resonance $\omega_{0}$, drastically alter the reflectivity of the surface. Reflectivity calculations are given for a wave incident normally upon infinite square lattices (of basis $l$ ) for three models: (1) air bubbles in a liquid fullspace, (2) air bubbles at a water/hard surface or water/ elastic plate interface, and (3) air-filled cavities in a rubber layer at such an interface. These illustrate the essential role of multiple scatter and, most interestingly, the possibility of reflectivity nulls for incident frequencies $\omega \simeq \omega_{0}$. For case ( 3 ), use of rubber constants given by earlier writers [Gaunaurd et al., J. Acoust. Soc. Am. 65, 573-594 (1979)] shows that it is theoretically possible to select values of $k l$ such that a hard wall (or an elastic plate) becomes fully anechoic for given $\omega \simeq \omega_{0}$. For the rubber types considered, the effective bandwidth of this effect ( $99 \%$ anechoicity) $\Delta \omega / \omega_{0}$ varies between 0.01 and 0.05 . [Work supported by ONR.]

\section{1:00}

B13. Acoustical images of a submerged aluminum sphere. Charles F. Gaumond, Brian H. Houston (Codes 5132 and 5136, Naval Research Laboratory, Washington, DC 20375-5000), and Sheila Woo (Sachs Freeman Associates, Landover, MD 20785)

Acoustical images using reflection tomography [P. B. Abraham and C. F. Gaumond, J. Acoust. Soc. Am. 82, 1303-1314 (1987) ] were made from 3-D acoustical scattering data. The data were taken from a 7.6-cm (3.0-in.) solid aluminum sphere using a semicircular hoop array with radius $0.85 \mathrm{~m}$, which rotated around the sphere. The element spacing in the hoop was $2^{\circ}$. The hoop was rotated in $2^{\circ}$ increments from the backscattered direction to the forward direction. The frequency range of the data was $2.7-25 \mathrm{kHz}$. These acoustical images were compared with analytically produced acoustical images.
11:30

B15. Sound scattering from a submerged finite cylinder with ribs. Philip J. Moser, Jian-Ren Yuan, and Herbert Überall (Department of Physics, Catholic University of America, Washington, DC 20064)

The effects of an almost-periodically spaced set of ribs on the scattering of sound from a submerged cylinder of finite length are investigated. The cylinder is either assumed soft (corresponding to a very thin air-filled shell) or rigid (corresponding to a heavy thick shell), and its scattering amplitude is obtained using the geometrical theory of diffraction. The rib scattering amplitude is found from curvature corrected results of the theory for a flat plate carrying a rib. Numerical results are shown for the case of soft cylinders; they exhibit the onset and emergence of rib effects over the background of scattering from the body of the cylinder.

\section{1:45}

B16. Diffraction tomographic interferometry, a new imaging technique. Woon S. Gan (Acoustical Services Pte Ltd., 29 Telok Ayer Street, Singapore 0104, Republic of Singapore)

A new imaging technique, diffraction tomographic interferometry (DTI), is proposed. Double-exposure DTI and time-averaged DTI are considered. The Born approximation is used to obtain the scattered wave and the filtered-backpropagation algorithm in the reconstruction. For double-exposure DTI, summation of the two tomograms gives the diffraction tomographic interferogram. Reconstruction gives a cross-sectional image covered with interference fringes. For time-averaged DTI, the intensity of the reconstructed image is expressed in terms of the characteristic function. This gives a cross-sectional contoured image of the object modulated by a system of interference fringes. For the proposed experimental setup for the double-exposure DTI, the interferogram of the object, a turbine blade in its unstressed state and stressed state, is recorded with the object illuminated from various directions. For the proposed experimental setup for the time-averaged DTI, a diffraction tomogram is exposed for a period of time by rotating the illuminating source around the object several times, during which the object executes a motion. 


\title{
Session C. Underwater Acoustics I: Acoustic Fields
}

\author{
John Perkins, Chairman \\ Code 5160, Naval Research Laboratory, Washington, DC 20375
}

Chairman's Introduction-8:00

Contributed Papers

\section{8:05}

C1. Three-dimensional matched-field processing with an eigenvalue method. Michael D. Collins, W. A. Kuperman, John S. Perkins, Laurie E. Tinker (Naval Research Laboratory, Washington, DC 20375), and John Glattetre (Norwegian Defense Research Establishment, P.O. Box 115, N-3191 Horten, Norway)

An eigenvalue method for three-dimensional matched-field processing with a vertical array has been developed for localization of multiple sources in range and depth (bearing can also be determined with the method if the environment is asymmetric). The method involves applying the Bartlett beamformer to the eigenvectors of the cross-spectral density matrix. This approach appears to be more effective than the standard eigenvalue approach involving beamforming on a linear combination of several eigenvectors. Eigenvalue beamforming methods are based on the orthogonal targets approximation [R. F. Gragg, NRL Rep. 9143, Naval Research Laboratory, Washington, DC (1989) ]. The spatial correlation over a horizontal array of two sources is small provided the sources are sufficiently separated in bearing. On the other hand, the spatial correlation over a vertical array of two point sources may be relatively large for some source locations (i.e., the Bartlett matched-field processor for a vertical array has relatively large sidelobes). However, simulations suggest that this effect does not seriously degrade the eigenvalue processing method for vertical arrays. Source motion will further enhance this processing method.

\section{8:20}

C2. A perturbation method for normal mode theory with variable velocity profile. M. F. Werby (School of Physics and Astronomy, University of Minnesota, Minneapolis, MN 55455 and NORDA, Numerical Modeling Stennis Space Center, MS 39529), and Paul Ellis (School of Physics and Astronomy, University of Minnesota, Minneapolis, MN 55455)

Normal mode theory is a very well-developed technique for predicting the propagation loss in the ocean for most stratified environments and velocity profiles, as well as some range-dependent environments. So why another method? The reason is that sometimes it is desirable to retain the simple trigonometric functions obtained from constant velocity profiles. This is usually done by employing the approximate scherne of using multiple layers in each of which the velocity profile is assumed constant. In this work, it is shown that this can be carried out nicely by representing the velocity profile in terms of a mini-max fit using Tschebyscheff polynomials and a first-order perturbation technique derived from Sturm-Liouville theory. The derivation leads to a compact closed expression that is easily prograrnmable. The results from the formulation are compared with other methods.

C3. Low-frequency acoustic tomography using matched field processing. I. A. Tolstoy (Code 5120, Naval Research Laboratory, Washington, DC 20375)
Can matched field processing be used to determine the sound-speed environment given a known source? Recent computations are very promising in spite of expected difficulties relating to the nonuniqueness of solutions. In the range-independent case, the essence of the approach is to find "the" sound-speed profile that will maximize the power seen at the array. This has resulted in a profile that is "close to" the true profile and found by means of an exhaustive search of the parameter space describing the family of potential profiles. The parameter space consists of the coefficients of the major empirical orthogonal functions (EOFs) computed from the covariance matrix of sound-speed profile samples. Subsurfaces of the parameter space are easily plotted and show well-defined, informative trends. Unfortunately, iterative techniques investigated so far have shown convergence difficulties in that they find local maxima which correspond to profiles which are not close to the true profile. Results will show the procedure sensitivity as a function of array length, source range, depth, and frequency. The key to success for the technique is the computation of "good" EOFs.

\section{8:50}

C4. Low-frequency acoustic tomography using matched field processing. II. A. Tolstoy (Code 5120, Naval Research Laboratory Washington, DC 20375)

Part I discussed the difficulties and successes of the MFP technique in a range-independent environment. Part II discusses progress to data (simulations only) concerning a range-dependent eddy environment. First, a sound-speed structure is computed as the sum of two Gaussian eddys. Next sample sound-speed profiles are computed along a selected radial and used to calculate the basic EOFs for the entire environment. The EOFs themselves are fairly insensitive to how the sampling is done although their coefficients can change significantly. Given a set of EOFs, plots of their coefficients as a function of range and cross range show welldefined, easily interpolated behavior. Moreover, only two EOFs are needed to describe a sound-speed profile to a high degree of accuracy. Results will be presented examining the sensitivity of MFP to changes in the coefficients. Regions of high sensitivity require frequent profile estimations and increased environmental sampling. Progress to date suggests that the large family of parameter values (at least two parameters, i.e., EOF coefficients, per profile) plus iterative difficulties as seen in Part I may require the application of computationally intensive techniques such as simulated annealing.

\section{9:05}

C5. Environmental mismatching effects on source localization processing in mode space. E. C. Shang and Y. Y. Wang (CIRES, University of Colorado/NOAA, Wave Propagation Laboratory, Boulder, CO 80303)

The performance degradation of the matched field processing (MFP) caused by environmental mismatching has been an intriguing subject. A great deal of study has been done by numerical simulations. In this paper, this problem is studied analytically. Analytic investigation is important because it reveals some of the physical insights and provides guidelines for 
a "self-coherent" scheme [H. Bucker, J. Acoust. Soc. Am. Suppl. 184, S19 (1988) ]. It has been illustrated that the source range information is associated with the modal phase difference and the source depth information is associated with the modal amplitude ratio when MFP is performed in mode space. Therefore, the modal wavenumber plays a key role in source localization. Mismatching effects can be investigated analytically by taking advantage of calculating the modal wavenumber error by modal perturbation theory. [Work supported by NOAA and NOSC.]

C6. Nonlinear bias of travel time in modal ocean acoustic tomography. $E$. C. Shang (CIRES, University of Colorado/NOAA, Wave Propagation Laboratory, Boulder, CO 80303)

In a previous paper [E. C. Shang, J. Acoust. Soc. Am. 85, 1531-1537 (1989) ], ocean acoustic tomography based on the adiabatic normal mode theory has been developed. Mesoscale structure can be inverted by using a linear relationship connecting the modal travel time perturbation and the sound-speed perturbation. This linear relationship is only an approximation due to the following two facts: (1) neglecting the difference between perturbed modal eigenfunction and the background model eigenfunction; (2) taking the first-order expansion of the "potential function" $\Delta K^{2}(2, r)$ to calculate the modal wavenumber perturbation. Comparing with ray tomography, item (1) is the term corresponding to the ray path perturbation. It has been found that item ( 1 ) is not significant and the problem caused by item (2) can be recovered by a "post-correction" procedure proposed in this paper. [Work supported by NOAA and ONR.]

\section{9:35}

C7. The normal mode theory with bottom elasticity and range dependence. Juan I. Arvelo (Code U25, Naval Surface Warfare Center, 10901 New Hampshire Avenue, Silver Spring, MD 20903-5000) and Herbert Überall (Department of Physics, Catholic University of America, Washington, DC 20064)

The theory of normal modes has been extended to accommodate the effects of shear waves in the elastic ocean floor. The effect of absorption has also been included as the imaginary component of the shear and compressional wavenumbers. A semi-infinite elastic bottom basement layer has been included and it was found that the absorption causes the wavenumber spectrum of the radiating modes to be inherently discrete, hence, the number of radiating modes is drastically reduced relative to the ones obtained with the deep false boundary. The range dependence of the acoustic properties and of the boundaries of the ocean has been included by a modified version of the adiabatic normal mode theory. Comparisons are made with experimental measurements of the transmission loss of underwater explosives and with results from Collins' HEPE mode. [Work supported by the Independent Research Board of the Naval Surface Warfare Center and by NORDA.]
C8. Effect of internal solitons on shallow-water sound propagation. Jixun Zhou, Xue-zhen Zhang, and Peter Rogers (School of Mechanical Engineering, Georgia Institute of Technology, Atlanta, GA 30332)

There have been many reports on observations of naturally occurring internal solitary wavetrains (solitons) in the coast zone, especially in the summer. The mechanism for the generation of these nonlinear internal waves has been investigated in the geophysics and fluid mechanics community. Little attention, however, has been focused on their effect on sound propagation with the exception of the work of Baxter and Orr, which was based on ray theory [L. Baxter and M. H. Orr, J. Acoust. Soc. Am. 71, 61-66 (1982) ]. In this paper, the parabolic equation (PE) model (IFD or PAREQ code) is used to numerically simulate the effect of internal solitons on low-frequency sound propagation in the coast zone. The results show that sound transmission loss is sensitive to soliton parameters (such as wavelength, amplitude, etc.) and wave packet parameters (position, number, and propagation direction). The results can be used to explain, at least partly, an interesting experiment phenomena: The frequency response of shallow-water sound propagation under the condition of a thermocline during the summer is often a strong function of space and time, and sound propagation over certain frequency ranges has an abnormal attenuation that cannot be explained by using conventional models of sound propagation. [Work supported by ONR.]

\section{0:05}

C9. Measurement of shallow-water sound transmission on the New Jersey continental shelf. Lynne Maiocco, William Carey (Naval Underwater Systems Center, New London, CT 06320), Edwin Parssinen (Kildare Corporation, 95 Trumbull Street, Suite D, New London, CT 06320), and James Doutt (Woods Hole Oceanographic Institute, Woods Hole, MA 02543)

Calibrated acoustic transmission measurements were made on the New Jersey continental shelf in the vicinity of the AMCOR 6010 borehole, an area with known geophysical properties. The water depth was 70 $m$ and the experiment was conducted under known oceanographic conditions as shown by measurements of salinity, temperature, and sound speed versus depth. The acoustic measurements were performed with a vertical array of 24 equally spaced hydrophones, one of which was on the bottom. A calibrated source was used to obtain transmission loss data to ranges of $26 \mathrm{~km}$ at discrete frequencies from 50 to $600 \mathrm{~Hz}$. The data from three hydrophones were processed for a 4-km range using techniques developed by Frisk and Lynch [J. Acoust. Soc. Am. 76, 205-216 (1984)] to yield wavenumber spectra. The transmission results of three hydrophones at 50 $\mathrm{Hz}$ were found to have an interference pattern clearly resulting from two modes. Low-frequency data $(20$ to $50 \mathrm{~Hz}$ ) were obtained at discrete locations from the buoy to yield values of normal boundary impedance. Transmission loss data were obtained at eight frequencies, 50 to $600 \mathrm{~Hz}$, along a constant depth and gradually changing depth radial. The constant depth radial results were compared to calculations performed with IFD-PE and SAFARI using a geoacoustic model based on measured geophysical properties. Predicted and measured levels generally agreed, however, differences in the computed and the measured modal interference patterns were observed.

\section{0:20-10:30}

Break

10:30

C10. Experimental results of sound propagation in a penetrable wedge with a fluid bottom. Stewart A. L. Glegg and Ian House (Center for Acoustics and Vibration, Department of Ocean Engineering, Florida Atlantic University, Boca Raton, FL 33431)
This paper will describe the results of a model scale experiment on sound propagation in a penetrable three-dimensional wedge with a fluid bottom. The wedge model is $1.2 \times 2.4 \mathrm{~m}$ and has a Mylar-kevlar membrane that separates two fluids (water and ethylene gylcol) with sound speeds of $1500 \mathrm{~m} / \mathrm{s}$ and $1700 \mathrm{~m} / \mathrm{s}$. The model is suspended in a large water tank so that reflections from the edges of the model are not significant. 
The experiment has concentrated on across slope propagation at several different downslope ranges, using a high-frequency pulse. The pulse spectra have been compared with Buckingham's theoretical model for sound propagation in a penetrable wedge. [Work supported by ONR.]

\section{0:45}

C11. The resolution of modal Doppler shifts in an acoustic waveguide. Hee Chun Song and Arthur B. Baggeroer (Department of Ocean Engineering, Massachusetts Institute of Technology, Cambridge, MA 02139)

A method for estimating the source velocity from the acoustic signal field propagating in a dispersive oceanic waveguide is presented. The signal is observed at an omnidirectional receiver in the presence of additive white noise. The source transmits a continuous-wave (cw) signal, and the source motion is assumed to be uniform (unaccelerated). The ocean is modeled as a waveguide that is horizontally stratified with an arbitrary sound-speed profile in the vertical. The acoustic ficld generated by a moving point source in terms of normal modes predicts that each mode has a different Doppler shift, which contrasts with a single Doppler shift in a homogeneous unbounded medium. The modes are considered to remain phase locked with each other, i.e., coherent. The source velocity, including the number of modes involved, can be estimated through resolving Doppler shifts. The method is a time-domain interpretation of a recently developed eigenstructure technique for multitarget direction finding with passive antenna arrays, in conjunction with smoothing preprocessing scheme to deal with coherent modes [Shan et al.. IEEE Trans. Acoust. Speech Signal Process. ASSP-33 (4) (1985) ]. Simulation results that illustrate the performance of the method are presented.

\section{1:00}

C12. Impulse response for propagation in the ocean sound channel. Stanley M. Flatté, John Colosi, Timothy F. Duda, Galina Rovner, and Jan Martin (Physics Department, University of California, Santa Cruz, CA 95064)

Using the SDSC/CRAY and the parabolic equation, pulse propagation was simulated in the ocean sound channel. Pulses with 200 frequencies between 10 and $50 \mathrm{~Hz}$ were synthesized, giving a pulse width of approximately $25 \mathrm{~ms}$, and a pulse repetition time of $5 \mathrm{~s}$. The wave fronts created by this technique have a simple and intuitive character, unlike the fields for single frequencies. Pictures of wave fronts out to $1000 \mathrm{~km}$, and possibly a movie, will be shown. Wave front reconstruction by means of a much faster calculation in terms of ray theory will be demonstrated. The effects of internal waves on the wave fronts will be described in terms of wave front corrugations, and the use of impulse measurements over vertical arrays to measure the properties of the internal-wave field will be discussed. [Work supported by ONR, Code 1125OA.]

\section{1:15}

C13. Modal matched-field processing with small aperture vertical arrays. G. B. Smith, G. M. Frichter, IV (Naval Ocean Research and Development Activity, Code 244, Stennis Space Center, MS 39529. 5004), C. Feuillade (Syntek Engineering and Computer Systems, Inc., 2101 East Jefferson Street, Rockville, MD 20852), and C. L. Byrne (Department of Mathematics, University of Lowell, Lowell, MA 01854)

In the past years, much attention has been given to mode space matched-field processing in shallow-water, low-frequency environments. In this type of situation, mode space matched-field processing has several advantages over conventional matched-field processing such as: lower processing dimension, betrer sidelobe rejection, and the ability to discriminate signal against modal noise. However, extraction of modal amplitudes from data by the method pioneered by Shang [J. Acoust. Soc. Am. 77, 1413-1418 (1985) ] is not very reliable for small aperture arrays. Attempts have been made to overcome this problem in order to make mode space matched-field techniques more applicable to actual physical environments [T. C. Yang, J. Acoust. Soc. Am. 82, 1736-1745 (1987)]. A fresh approach is brought to this problem here using computer simulations of matched-field processing in a Pekeris waveguide to demonstrate a new method for extracting modal amplitudes from data. These simulations demonstrate that this new method of modal amplitude extraction allows successful mode space matched-field detection and localization at array apertures significantly smaller than those allowed by previously used algorithms. Finally, the algorithm will be discussed in the general context of dimensional reduction techniques.

\section{1:30}

C14. Combined oceanographic and acoustic modeling. Michael Porter, Steve Piacsek, Laurel Henderson, and Finn Jensen (SACLANT Undersea, Research Centre, I-19026 La Spezia, Italy)

An important class of oceanographic models is the mixed layer models that, simply stated, provided predictions of mixed layer depth and temperature. These predictions take into account the surface winds that drive the mixing and the solar radiation that heats the mixed layer. As interest has increased in using such models as a step in the process for making acoustic forecasts or nowcasts, a recurring question has been that of what information is needed for accurate acoustic predictions. For instance, how precisely do mixed layer depths need to be calculated? Is mixed layer temperature important? These and other questions are addressed through a parameter study using synthetic sound-speed profiles as input to an acoustic model. The implications are demonstrated in a somewhat more concrete form in a two-step process: The SSP is predicted using particular mixed layer models (Niiler, Mellor-Yamada, Garwood, etc.) and passed to an acoustic model to provide transmission loss calculations. The results are also compared to predictions based on both measured and historical SSP measurements.

\section{$11: 45$}

C15. The transport coherence of acoustic fields as a criterion for range dependence in an ocean channel. B. G. Hurdle (Naval Research Laboratory, Code 5103, Washington, DC 20375)

A paper was presented [B. Hurdle, J. Acoust. Soc. Am. 55, 452 (1974) ] on the nature of acoustic interference fields in a geometric dispersive ocean channel. The structure and multiple components of the fields as a function of the velocity of the source, the geometry of the boundaries, and the sound-speed structure of the channel were discussed. This paper reviews the nature of the fields and, based on the field characteristics, provides a criterion for determining the degree of range dependence of an ocean channel. This criterion is based on the transport coherence of the field and is measured in terms of the correlation coefficient of the transported field as a function of the separation distance between two receivers as the field is transported the distance between them. The difference in the transport coherence and the coherence in the field for beam forming will be discussed. Some other applications of this concept will also be discussed.

\section{2:00}

C16. The use of neural networks in acoustic coherence measurements. Mark J. Beran (Department of Electrical Engineering. Catholic University of America, Washington, DC 20064)

The intensity distnbution of an incoherent target may be determined from coherence measurements. It is shown here how a neural network may be developed to solve this inverse problem. The effect of random phase variations resulting from ocean temperature fluctuations is considered in the formulation. 
Session D. Psychological Acoustics I: Temporal Effects and Binaural Phenomena

\author{
D. Wesley Grantham, Chairman \\ Bill Wilkerson Hearing and Speech Center, 1114 19th Avenue, South, Nashville, Tennessee 37212
}

Contributed Papers

D1. Comodulation masking release (CMR) using coherent complex envelopes. Mark P. Haggard and Deborah A. Fantini (MRC Institute of Hearing Research, University Park, Nottingham NG7 2RD, England)

In comodulation masking release (CMR), thresholds for a signal masked by a narrow band of noise are improved when a second, flanking, noise band is added which has a similar amplitude envelope to that of the masking band. The parameters of "similarity" are many; this study addresses sharing of frequency components in complex modulations. The following was determined: (a) whether the auditory system may extract components of complex modulation in order to achieve a CMR; (b) whether a "missing fundamental" of a complex modulation matches up with a present modulation component to provide a CMR; and (c) whether the difference or beat frequency of a complex modulation may provide a CMR, for both harmonic and inharmonic complexes. The results indicate a small but significant CMR with complex modulation; the reduction in CMR by comparison with sinusoidal modulation may reflect modulation-modulation masking. Insignificant CMRs were obtained where components would need to be separated to register envelope similarity between differing complex modulations.

\section{8:45}

D2. The role of envelope information in CMR tasks. Deborah A. Fantini (MRC Institute of Hearing Research, University Park, Nottingham NG7 2RD, England)

In comodulation masking release (CMR), thresholds for a signal masked by a narrow-band noise are reduced when additional noise is present, provided that the additional noise has the same amplitude envelope fluctuations over time as the original masking band. Recently, some speculation has appeared in the literature as to the specific source of information which provides the reduction in masked threshold. In the present work, the importance of changes in the envelope of the masker band, due to the addition of the signal at threshold, in providing the CMR, has been investigated. First, CMR thresholds were collected in sinusoidally amplitude-modulated noises which varied either in the phase relationship of their envelopes or in their modulation depth. Then, envelope discrimination (ED) thresholds were collected for changes in envelope phase disparity and for changes in modulation depth per se. The data indicate that the patterns of CMR thresholds with envelope phase disparity and with modulation depth vary differently from that of the ED thresholds. A simulation was then conducted in which the stimulus waveforms were processed through a model that includes an auditory filter [equivalent rectangular bandwidth (ERB) filtering], a square-law nonlinearity, and a sliding temporal window [an equivalent rectangular duration (ERD) filter]. The envelopes were then extracted from the processed waveforms to determine what actual changes in the envelopes were occurring in the signal intervals in both CMR and ED. The results of this waveform analysis indicate that discriminability of envelope changes due to the addition of the signal at threshold in the CMR task are insufficient to explain CMR.
D3. A comparison of comodulation masking release and profile analysis. Deborah A. Fantini (MRC Institute of Hearing Research, University Park, Nottingham NG7 2RD, England), Gregory P. Schooneveldt, and Brian C. J. Moore (Department of Experimental Psychology, University of Cambridge, Cambridge, England)

Large reductions in signal thresholds may be observed under stimulus conditions in which there is common information in a number of auditory channels or critical bands. In comodulation masking release (CMR) tasks, a reduction in the threshold of a signal in a narrow-band noise occurs when a flanking or cue band of noise is also present, provided that the flanking band has an amplitude envelope which is correlated with that of the masker band. This reduction in threshold occurs in dichotic conditions, where the flanking band is presented in the opposite ear to the signal-plus-masker. In profile analysis (PA), the overall level is randomized from one stimulus to the next. The threshold for detecting an increment in level of a single component is reduced when flanking components equal in amplitude to the pedestal are added. In general, this effect has not been found when the flanking components are presented dichotically. The present work examines the extent of the similarity, and divergence, between CMR and PA. For the PA conditions, a five-component sinusoidal complex was used; its level was varied randomly over a $40-\mathrm{dB}$ range. For the CMR conditions, each of the five components was $100 \%$ sinusoidally amplitude modulated at a $10-\mathrm{Hz}$ rate. Two intermediate conditions of PA without the level variation, and CMR with the level variation, were also run. All four conditions were run monaurally and dichotically. A reference condition in which only one masking component was present was also used. For PA conditions when the stimulus level was randomized, thresholds in the monaural and dichotic conditions were 16 and $11 \mathrm{~dB}$ lower than in the reference condition, respectively. By contrast, for the CMR conditions, whether the stimulus level was randomized or not, thresholds in the reference, monaural and dichotic conditions differed by little more than $3 \mathrm{~dB}$; i.e., no CMR was observed. Further experiments are now in progress to determine why a CMR was not observed in these conditions.

\section{9:15}

D4. Spectral fusion based on coherence of amplitude modulation. Stanley Sheft and William A. Yost (Parmly Hearing Institute, Loyola University, 6525 North Sheridan Road, Chicago, IL 60626)

The ability of listeners to attend to a subset of components ( the target) of a tonal complex was investigated using a forced-choice procedure. The stimulus was an eight-component tonal complex. The components of the target subset ( $n=1,2$, or 3 components) were distinguished from the complex by coherent amplitude modulation (AM). Each trial of the cued 2IFC task was preceded by a presentation of the coherently modulated target components in isolation. For each interval of a trial, either the target or an equal number of nontarget components shared the coherent AM. Subjects were required to detect the interval in which the target 
components were coherently modulated. The remaining components of the complex were all either modulated at different rates, modulated with a random shift of the target-modulator phase angle, or not modulated. Performance was measured as a function of the number of target components, the harmonic relationship among the target components, and the harmonic relationship between the target and nontarget spectral groups. With coherent AM of harmonic target components, the increment in $d^{\prime}$ with increasing $n$ exceeded predictions based on the combination of independent sources of information, suggesting that these stimuli may be processed as an entity by the auditory system. [Work supported by NINCDS.]

\section{9:30}

D5. Sensitivity to envelope coherence. Virginia M. Richards, David M. Green, and Zekiye A. Onsan (Psychoacoustics Laboratory, Department of Psychology, University of Florida, Gainesville, FL 32611)

In a 2IFC paradigm, listeners indicated whether two, simultaneously presented, sinusoidally amplitude-modulated tones had envelopes that were in-phase rather than out-of-phase. The depth of modulation, $m$, necessary for $70 \%$ correct decisions was determined. Thresholds were obtained for carrier frequency separations of $2 / 3$ and $4 / 3$ of an octave, for geometric means of the two carriers ranging from $500-8000 \mathrm{~Hz}$, and for modulation rates varying from 4 to $320 \mathrm{~Hz}$. Increasing the frequency separation of the two carriers from $2 / 3$ to $4 / 3$ of an octave had little effect, reducing sensitivity by at most $5 \mathrm{~dB}$. Similarly, thresholds were nearly independent of the geometric mean of the tonal carriers. Plotting detection threshold as a function of modulation rate yielded curves that were roughly low pass in character: For modulation rates less than $100 \mathrm{~Hz}$, performance levels were approximately constant, and, for modulation rates less than $100 \mathrm{~Hz}$, sensitivity to envelope phase deteriorated with increased modulation rate. [Work supported by the Air Force Office of Scientific Research and the National Institutes of Health.]

\section{9:45}

D6. Phase effects in masking of one modulation frequency by another. Elizabeth A. Strickland and Neal F. Viemeister (Department of Psychology, University of Minnesota, Minneapolis, MN 55455)
Masking of one modulation frequency by another was measured in a 3IFC task. The carrier was broadband noise at a spectrum level of $30 \mathrm{~dB}$ SPL. This was amplitude modulated by a sinusoidal masker or a sinusoidal signal plus a sinusoidal masker. Masker modulation depth was fixed at $25 \%$ or $50 \%$, and masker frequencies ranged from $2-800 \mathrm{~Hz}$. Threshold signal modulation depth was measured for signals of $4,16,100$, and 400 $\mathrm{Hz}$. The masking functions showed broad tuning for modulation frequency consistent with that seen by Bacon and Grantham [ J. Acoust. Soc. Am. 85, 2575-2580 (1989) ]. For example, for a signal modulation frequency of $100 \mathrm{~Hz}$, the amount of masking only varied over $5 \mathrm{~dB}$ for masker modulation frequencies from 40 to $140 \mathrm{~Hz}$. Thresholds were phase dependent for masker frequencies of half, equal to, or twice the signal frequency. For equal signal and masker frequency, thresholds were consistent with detection of differences in envelope power, independent of signal/masker phase. Results are discussed in terms of models of modulation detection. [Work supported by grants from NINCDS (NS12125) and NICHHD (HD-07151).]

\section{$10 ; 00$}

D7. Transition from simultaneous to forward masking III: Phase effects. Daniel L. Weber (Department of Psychology, Wright State University, Dayton, OH 45435)

The relative phase of a sinusoidal masker and signal necessarily has a large effect upon the signal power required for a simultaneous-masked threshold but has no effect on the forward-masked threshold. Threshold for a 20 -ms (20-ms raised cosine ramps with no steady state produced a signal $20 \mathrm{~ms}$ long measured at the half-voltage points on the envelope), 1$\mathrm{kHz}$ sinusoid, masked by a $300-\mathrm{ms}$ (no ramps), $1-\mathrm{kHz}$ sinusoid, was measured as a function of the signal's temporal position to examine how the phase effect disappeared in the transition from simultaneous to forwardmasking conditions. Although listeners differ, the phase effect remains unchanged at least until $10 \%$ of the signal energy is subject to forward masking and is substantially reduced, if not eliminated, when $50 \%$ of the signal is subject to forward masking. These results are interpreted to indicate that forward masking tends to be the more sensitive detection process when a signal is subject to both simultaneous and forward masking.

\section{0:15-10:30}

Break

10:30

D8. The effect of onset asynchronies between components on the ability to detect a delayed component embedded in a diatic complex. $M$. A. Stellmack and R. H. Dye (Parmly Hearing Institute, Loyola University, 6525 North Sheridan Road, Chicago, IL 60626)

The purpose of this study was to extend the author's previous research on the processes that produce interference when observers attempt to lateralize a target component in the presence of other components. The present study examines the effect of an onset asynchrony between an interaurally delayed target (IDT) and diotic "distractor" components. Threshold $\Delta$ IDTs were measured for a $753-\mathrm{Hz}$ tone presented against a diotic background of six additional components with $\Delta f$ 's of 10,50 , or $100 \mathrm{~Hz}$. Onset asynchronies between the distractors and target were 0,50 , 100 , or $200 \mathrm{~ms}$, with the distractors always gated on first. The duration of the target was always $200 \mathrm{~ms}$. The duration of the distractors was such that they remained on for the duration of the target (all components were gated off simultaneously). The rise/decay time of all components was 10 $\mathrm{ms}$ and each component was presented at $55 \mathrm{~dB}$ SPL. The largest thresholds were obtained when $\Delta f$ was $50 \mathrm{~Hz}$ at each onset asynchrony. One subject showed no effect of onset asynchrony and the second subject showed no effect for $\Delta f=100 \mathrm{~Hz}$, with thresholds increasing with increasing onset asynchrony when $\Delta f$ was 10 or $50 \mathrm{~Hz}$. These results show that onset asynchrony between components does not diminish the effects of spectrally synthetic binaural processing. [Work supported by NIH.]

\section{$10: 45$}

D9. Onset coding of spatial information in trains of identical transients. Kourosh Saberi and David R. Perrott (Psychoacoustics Laboratory, California State University, Los Angeles, CA 90032)

In a study of the effects of onsets in lateralizing bursts of noise, Tobias and Schubert [J. Acoust. Soc. Am. 31, 1595-1605 (1959) ] demonstrated that, depending on the duration of the ongoing noise burst, it takes anywhere between 4 to 35 times as much onset interaural time disparity to offset ongoing disparities. They, therefore, demonstrated that onsets do not necessarily provide the major cue to auditory localization. In the present study, onset and ongoing information in a lateralization paradigm is set in spatial conflict to determine the amount of onset interaural difference of time (IDT) necessary to offset ongoing IDTs of transient trains of various durations. The major difference between this study and the Tobias 
and Schubert study was that this study used identical transients [e.g., interclick interval (ICI) $=1.5$ to $2 \mathrm{~ms}$ corresponding to a $500-$ to $750-\mathrm{Hz}$ tone] and not noise bursts. Unlike the Tobias and Schubert study, it was found in this study that a single dichotic click could easily lateralize a contralateral transient train of up to 200 clicks; producing the sensation of a tone lateralized to the position of the initial event. Various ICIs and ongoing durations were tested. In such a context, the precedence effect, the Franssen illusion, and the formation of auditory objects will be discussed. [Work supported by NSE and NIH.]

\section{1:00}

D10. Minimum audible movement angles for sources varying in both elevation and azimuth. David R. Perrott and Kourosh Saberi (Psychoacoustics Laboratory, California State University, Los Angeles, CA 90032)

A minimum audible movement angle (MAMA) experiment was performed in a "simulated motion" paradigm which allowed travel along vertical, horizontal, or oblique paths. Velocities of travel ranged from 1.8$230 \%$ s. With motion restricted to the horizontal plane, MAMA thresholds increased from about $1.7^{\circ}$ at the lowest velocity $(1.8 \%$ s) to roughly $10^{\circ}$ at a simulated velocity of $230^{\circ} / \mathrm{s}$. With the sound traveling on an oblique plane $\left(45^{\circ}\right.$ rotation above the horizontal ) MAMA thresholds generally matched those of the horizontal condition. When the motion was restricted to the vertical plane (the array was rotated $90^{\circ}$ from the horizontal) MAMA thresholds were substantially higher at all velocities than thresholds observed in the other array orientations, often exceeding $8^{\circ}$ even at low velocities. Post hoc tests conducted at oblique planes $80^{\circ}$ and $87^{\circ}$ indicated that the slightest deviation from the vertical, had a significant impact on MAMA thresholds. A deviation of $10^{\circ}$ from the vertical orientation was sufficient to reduce thresholds to those obtained when the sound moves on the horizontal plane $\left(0^{\circ}\right.$ rotation above horizontal). These results suggest: (1) the ability to detect motion is essentially independent of the path traveled with one noted exception; sources traveling on an absolute vertical dimension, and (2) detection of the direction of travel is possible with azimuth changes of only a few tenths of a degree if concurrent changes in elevation are available. Some implications of these results are discussed. [Work supported by NSF and NIH.]

\section{1:15}

D11. Masking in simulated free-field. Pierre $L$. Divenyi (Speech and Hearing Research, V.A. Medical Center, Martinez, CA 94553)

R. W. Gatehouse [Proc. 12th I.C.A. 2, B2-7 (1986); J. Acoust. Soc. Am. Suppl. 182 , S108 (1987) ] reported a free-field masking effect that appears anomalous on the surface: $A$ pure-tone probe positioned at a nonzero azimuth is maximally masked by a broadband noise when the azimuthal position of the masker does not coincide with that of the probe. In the present experiments, similar results were obtained with stimuli presented in a simulated free-field using head-related transfer functions measured in an artificial head [J. Blauert and P. Laws, Acustica 29, 273$277(1973)$ ], with a $3-\mathrm{kHz}$ probe located at a $45^{\circ}$ left azimuth and a whitenoise masker positioned at either $45^{\circ}, 30^{\circ}$, or $20^{\circ}$ left azimuths. Maximum masking was observed at the $30^{\circ}$ noise location. This nonmonotonic masking effect may not be due to anything but the head-induced diffraction patterns characteristic to the $3-\mathrm{kHz}$ range: Measurement of the noise power (at the output of the transfer functions) inside the 1/3-oct band surrounding the probe showed that, as the noise was displaced from the $45^{\circ}$ left to the $30^{\circ}$ left position, the decrease of the noise level in the left ear was less important than the increase in the right ear. Adding this variablelevel noise to the fixed signal should thus result in monaural masked thresholds that are not appreciably different in the left (i.e., the near) ear but that monotonically increase with decreasing left azimuths in the right (i.e., the distant) ear. These predictions were, indeed, confirmed by results of masking experiments using the same stimuli presented monaurally in the two ears. [Work supported by the Department of Veterans Affairs.]

\section{$11: 30$}

D12. Effects of temporal position on the detectability of interaurally uncorrelated noise. Martin E. Rickert and Donald E. Robinson (Department of Psychology, Indiana University, Bloomington, IN 47405)

Listeners' ability to detect an interaurally uncorrelated segment of noise within binaural broadband noise bursts was measured as a function of the temporal position of the uncorrelated noise. Psychometric functions were obtained by varying the duration of uncorrelated noise. The results indicate that detectability improves as the duration of uncorrelated noise is increased. Temporal position affected detectability only at the shortest durations of uncorrelated noise tested; a segment positioned either at the end or at the beginning of the burst is more detectable than one in the middle. This result is not easily accounted for by models of binaural processing. For example, the EC model [N. I. Durlach, J. Acoust. Soc. Am. 35, 1206-1218 (1963)] assumes that performance is determined by the power of the difference in the waveforms in the two auditory channels. Since power is computed over the total stimulus duration, it is independent of the temporal position of the uncorrelated noise. The relationship of the current findings to those for the discriminability of diotic noise stimuli will be discussed. [Work supported by AFOSR.]

\section{1:45}

D13. Binaural detection with reproducible short-duration narrow-band maskers. E. Colburn and H. Steven Colburn (Boston University, 48 Cummington Street, Boston, MA 02215)

Hit and false alarm rates were measured for detection of a $500-\mathrm{Hz}$ tone target in each of ten samples of 1/3-oct noise centered at $500 \mathrm{~Hz}$ with a duration of $10 \mathrm{~ms}$, for both NoS $\pi$ and NoSo conditions. Two target waveforms were used, differing only in their phase relative to the masker. Three subjects are being tested, but extensive data is available from only one subject so far. Also, the NoSo experiment has been done for only one target phase. The NoSo hit rates correlate very well with the energies in the waveforms. For NoS $\pi$, the effect of target phase on hit rate is substantial, with almost no correlation between the rates for the two target phases. This is very different from earlier results with long duration waveforms [e.g., Gilkey et al., J. Acoust. Soc. Am. 78, 1207-1219 (1985)]. Neither models based on lateral position nor models based on the sum of the squares of the interaural differences correlate well with the data. Since subjects report multiple images and the interaural differences sometimes change substantially over the 10-ms duration, models that process the first and second halves of the waveforms are analyzed separately. [Work suported by NINCDS.] 


\title{
Session E. Structural Acoustics and Vibration I: Structureborne Noise in Nonhomogeneous Solids
}

\author{
Miguel C. Junger, Chairman \\ Cambridge Acoustical Associates, Inc., 80 Sherman Street, Cambridge, Massachusetts 02140
}

Chairman's Introduction-8:30

\author{
Invited Papers
}

\section{8:35}

E1. Steady-state and transient waves in random composites. Abraham I. Beltzer (Holon Institute for Technological Education affiliated with Tel-Aviv University, Tel-Aviv, Israel and Laboratorie de Modélisation en Mécanique, Université Pierre et Marie Curie, France)

A causal approach is applied to investigate harmonic waves in random media, which exhibit both scattering and viscoelastic losses. Since this approach allows for evaluation of the effective wavenumber for the entire frequency interval, the transients may then be investigated by the classical Fourier method. A particular example deals with the scattering model by $\mathrm{Wu}$, and the viscoelastic model by Azimi, which find applications in seismology. Numerical results for a transient response of this $\mathrm{Wu}$-Azimi model are presented. The pulse reinforcing and delaying effects of multiple scattering are shown to be interrelated through a causality constaint.

E2. The construction of dynamic effective medium theories (EMT) for inhomogeneous fluid or elastic continua. G. C. Gaunaurd (Naval Surface Warfare Center, Research Department (R43), White Oak, Silver Spring, MD 20903-5000)

A deterministic technique is described that predicts the dynamic (i.e., frequency-dependent) effective properties of inhomogeneous media. Situations are considered in which a host medium sustaining a distribution of spherical inhomogeneities is either fluid or solid. In the Rayleigh region (viz., $a / \lambda<1$ ) in which most effective medium theories (EMTs) hold, inhomogeneities of any shape can be assumed spherical without much error. However, assuming them to be penetrable implies that they realistically admit interior fields, which are coupled to the exterior ones by stress and displacement boundary conditions. The outcome of dynamic EMTs is a set of expressions to predict the frequency dependence of the effective (sound) wave speed $\bar{c}_{\mathrm{eff}}$, and the effective (sound) wave attenuation $\tilde{\alpha}_{e r f}$ in the mixture. These quantities emerge from the real and imaginary parts of the effective wavenumber $\tilde{k}_{\text {eff }}$, which are connected by Kramers-Kronig type relations, as has been discussed by Dr. Beltzer. An important consideration for the practical usefulness of an EMT is its simplicity. Often, the resulting predictive expressions are so cumbersome that they can only be evaluated by means of quite formidable computer codes. The principal EMT to the discussed here, yields closed-form simple predictive expressions for $\overline{\mathcal{C}}_{\text {eff }}$ and $\tilde{\alpha}_{\text {eff }}$, which, in many instances, are valid over significantly broad frequency bands. These expressions reduce to (and contain) almost all existing earlier results for static $(f \rightarrow 0)$ cases. The (present form of this) EMT is constructed by exploiting the presence of the monopole resonance of the inhomogeneities, and thus it works best in the cases in which this resonance is dominant. This EMT has to be further refined to account for cases where the dipole resonance is dominant. In this regard, Dr. Martinez, in a later lecture, will discuss an approach to more accurately deal with heavy/rigid inclusions in a rubberlike matrix. [Work supported by NSWC and ONR.]

E3. Some acoustic properties of layered-composite plates. Y. F. Hwang and P. J. Zoccola (David Taylor Research Center, Bethesda, MD 20084)

This paper discusses a method of calculating the modes of wave propagation in a composite plate. A composite plate consists of layers of different elastic and/or viscoelastic materials. A novel three-layer sandwich plate, which utilizes a light weight soft core sandwiched between stiffer septums ( to increase flexural rigidity without severe weight or cost penalty), will be closely examined in terms of its antisymmetrical (flexural or bending) and symmetrical (longitudinal or bulge) modes of wave propagation. The dispersion relationship and the coincidence frequency of the flexural wave will be discussed. Effects of fluid loading and some other acoustic properties of the composite plate, such as the transmissibility and reflectivity of sound, will also be discussed. 
E4. Pulse propagation in irregularly heterogeneous waveguides-Some model calculations. John J. McCoy and John Tanzosh (School of Engineering and Architecture, Catholic University of America, Washington, DC 20064)

Numerical simulations are presented of the propagation of pulses through a series of idealized one-dimensional waveguides, both isolated and coupled. The results are discussed in the context of determining what aspects lead to predictive modeling in terms of statistical moments; what ergodicities can be argued to allow predictions for an ensemble to apply to a single manifestation of the ensemble; what physics need to be accommodated in mean field formulations; what are the necessary structures of these formulations; etc. The emphasis of the simulations is to illustrate phenomena, as distinct from quantitatively reproducing expected results of physical experiments for specific application. [Work supported by ONR.]

\section{0:15}

E5. The damping of beam vibrations by viscoelastic layers bearing pellets: Theory and experiment. Rudolph Martinez (Cambridge Acoustical Associates, Inc., Cambridge, MA 02140)

A number of powerful solutions for the scattering action of spherical inclusions in extended elastic media are available in the literature. These analyses are relatively general and thus are usually too complex to provide direct insight into the relations between their many design variables. Rather, they often require parametric studies based on numerical results. The present development takes instead a simpler view that focuses on the two only possible types of motion of a single underformable pellet in an incident field containing dilatational and shear waves: a translational response for the dilatational case and both pellet translation and rotation for the shear wave field. The analysis emphasizes the fact that each type of motion has an associated broad resonance and antiresonance pair about which energy dissipation is maximized. These frequencies depend on pellet mass and moment of inertia, and may therefore be controlled to increase damping within given bands of interest. Our study of flexural vibrations applies the single-pellet theory to predict the effective loss factor of beams having constrained and unconstrained layers bearing undeformable inclusions, and compares the model's results to measurements.

\section{Contributed Papers}

\section{$10: 40$}

E6. Transient propagation of a one-dimensional wave in a harmonically inhomogeneous medium. Hyun Sil Kim and Jerry H. Ginsberg (School of Mechanical Engineering, Georgia Institute of Technology, Atlanta, GA 30332)

This paper considers the transient propagation of a one-dimensional dilatational wave in a spatially harmonic inhomogeneous medium of semi-infinite extent. A regular perturbation analysis of the response, suitable when the amplitude of the inhomogeneous deviation is small, reveals that inhomogeneity causes backscattering in the second-order term. A temporally harmonic excitation generating a signal whose wavelength is twice the periodicity of the inhomogeneity is shown to lead to a secondorder standing wave behind the wave front whose amplitude growth ultimately invalidates the solution. The frequency at which this situation occurs matches the frequency at which Floquet theory predicts instability in the steady-state response. A finite-difference analysis based on the method of characteristics confirms the early-time validity of the perturbation solution. It also reveals that the late-time signal is essentially a standing wave in the region extending outward from the transducer to within several wavelengths of the wave front, whereas it most closely resembles a nondispersive propagating wave in the region of the wave front. [Work supported by NSF.]

\section{0:55}

E7. Airborne sound transmission across resilient mounts. Michael F. Shaw (Naval Sea Systems Command, Code 55N, Washington, DC 20375) and Courtney B. Burroughs (Applied Research Laboratory, Pennsylvania State University, State College, PA 16801)
The airborne transmission loss across resilient mounting systems is investigated by developing an analytic model of the transmission of vibration from a point-driven, simply supported plate to a parallel infinite plate through the air separating the plates. Predictions of airborne transmission losses are compared with transmission losses through resilient mounts to quantify the role of the airborne path in transmission losses through welldesigned, single-stage resilient mounting systems. The sensitivity of the airborne transmission losses to separation distance, thickness of the receiving plate, size of the driven plate, and damping in the plates are presented.

\section{1:10}

E8. Embedded fiber-optic acoustic transducer and sensor arrays for acousto-ultrasonic evaluation of materials. Chris Thompson and Richard O. Claus (Fiber and Electro-Optics Research Center, Virginia Polytechnic Institute and State University, Blacksburg, VA 24061)

A novel technique for generating acoustic pulses inside a material and then sensing the signal again within the material is proposed. A hollow core fiber-optic waveguide is constructed with periodic solid "plugs" fused in place. The optical fiber is embedded in a solid material, and laser light is sent down the hollow core fiber. The laser energy is absorbed and attenuated at the plug locations, causing localized heating that will then act as a source of ultrasonic waves due to the thermoelastic mechanism. Fiber-optic sensors are also embedded to receive the acoustic wave fronts. Any material disturbances in the path of the acoustic waves will effect the velocity and attenuation of the wave, which can be quantified using the stress wave factor (Vary $e t a l$.). The fiber-optic transducer/receiving pairs can be located anywhere within or upon a material or structure and can be used to detect damage in situ. This technique is particularly valuable in assessing damage in composite materials. 

radiation patterns showing the influence of stiffener size and location.

E9. Analysis by variational principles of the vibration and acoustic radiation of a vibrating elastic plate with circumferential stiffeners. Jerry H. Ginsberg and James McDaniel (School of Mechanical Engineering. Georgia Institute of Technology, Atlanta, GA 30332)

This study concerns the effects of circumferential ring stiffeners on the vibration and acoustic radiation of a submerged circular elastic plate. The disk, which is excited by an arbitrary axisymmetric harmonic force, may be supported by either a rigid annulus or by an infinite baffe. The analysis employs variational principles [J. H. Ginsberg and P. T. Chen, J. Acoust. Soc. Am. Suppl. 1 82, S1 (1987) ], in which surface pressure and velocity are represented by assumed model functions. The presence of stiffeners alters the kinetic and potential energies of the plate, but it does not affect the formulation of the fluid-structure interaction. Two structural formulations are addressed, differing by whether the connection between the plate and the stiffeners is implicitly or explicitly enforced. The results for surface response are used in conjunction with the Kirchhoff-Helmholtz [Work supported by ONR.]

\section{1:40}

E10. Noncompressible liquid layer elasticity phenomenon. Vladimir A. Babeshko (Department of Mathematical Modelling. Kuban State University, Krasnodar, 350640, USSR )

A vibration process localization phenomenon, arising in semi-infinite regions, is known to exist in media other than solid deformable media. An infinite layer of an ideal incompressible liquid $r+h$ deep is in contact with an elastic plate, vibrating at the frequency $\omega$. Analysis of this system shows that for some vibrating plate size and mass, energy propagates to infinity unattenuated. The conditions favoring such vibration processes when unrestricted resonances can arise are presented [V. A. Babeshko, Lett. J. Technol. Phys. 14 (8) (1988)].

\title{
Session F. Musical Acoustics I: Strings, Winds, and Harmony
}

\author{
Thomas D. Rossing, Cochairman \\ Department of Physics, Northern Illinois University, DeKalb, Illinois 60115 \\ Uwe Hansen, Cochairman \\ Department of Physics, Indiana State University, Terre Haute, Indiana 47809 \\ Chairman's Introduction-9:00
}

Contributed Papers

9:05

F1. Determination of a string's impulse and step responses by Laplace transformation; relationships to exciting mechanisms. Ernst Terhardt (Institute for Electroacoustics, Technical University, D-8000 Munich, West Germany)

The excitation of a musical string is intimately dependent on the string's dynamic behavior-i.e., of the string alone-at the point of excitation. With resonable approximation that behavior can essentially be regarded as that of a linear system. An elegant way to describe it is by Laplace transformation. On the basis of the familiar equivalence of string and homogeneous electrical transmission line, the string impedance at the excitation point is expressed in Laplace-transform notation. This serves as a basis for getting the frequency characteristics of the impedance (magnitude and phase), and, by inverse Laplace transformation, the impulse and step responses of force and velocity, respectively. These characteristics of the string alone prove to be very helpful in discussing the interaction between a string and any exciting device such as a bow or piano hammer.

9:20

F2. Precision measurement of violin string modes. Bernard Ricca, Gabriel Weinreich, and Nicholas R. Michael (Randall Laboratory of Physics, University of Michigan, Ann Arbor, MI 48109)
Following a suggestion of Benade (private communication) that small inharmonicities in the mode frequencies of a string may have important effects on its bowing response, a method has been developed for precisely measuring the (complex) normal frequencies and polarizations of a string as mounted on a violin, with the aim of determining, among other things, whether the violin maker's adjustments of bridge and soundpost are systematically reflected in the string's inharmonicity. The procedure, which uses acoustic excitation and a two-dimensional photoelectric detector of string motion, is based on earlier work [E. B. Arnold and G. Weinreich, J. Acoust. Soc. Am. 72, 1739 (1982) ] but uses a higher frequency resolution $(0.125 \mathrm{~Hz})$ and a least-squares fitting of the resonant string response. Some resulis on the shift of string frequencies due to controlled loading of the bridge will be presented. [Work supported by NSF.]

\section{9:35}

F3. Low pitched notes associated with large bowing forces. R. J. Hanson, A. J. Schneider (Physics Department, University of Northern Iowa, Cedar Falls, IA 50614-0150), and F. W. Halgedahl (School of Music, University of Northern lowa, Cedar Falls, IA 50614)

For bowing forces greater than the Schelling maximum [J. Shelling, J. Acoust. Soc. Am. 53, 26-41 (1973)] there are various periodic motions possible with periods much longer than any natural period of the string [M. E. McIntyre, R. T. Schumacher, and J. Woodhouse, J. Acoust. Soc. 
Am. 74, 1325-1345 (1983)]. Aperiodic motion with a corresponding raucous sound are, however, much more readily produced with a large bowing force. If care is exercised by the player, a repetitive waveform with a nonraucous sound for very large bowing forces can be maintained for as long as several seconds. The resulting pitch that is heard is in agreement with the fundamental frequency of the harmonic series obtained with a frequency analyzer. This is not the small flattening effect with moderately high bowing force analyzed by McIntyre $e t$ al. but is a large effect producing pitches typically ranging from a musical 6th to a musical 12th below the normal pitch. Systematic patterns of the measured frequencies as a function of normal string frequencies have been observed and possible interpretations will be presented.

\section{9:50}

F4. Use of one-dimensional photodiode arrays to detect string vibration. Takashi Nakamura, Lawrence L. Dirkes, and Roger J. Hanson (Department of Physics, University of Northern Iowa, Cedar Falls, IA 50614-0150)

A device has been developed to measure the $x-y$ amplitudes of vibration of a string and to facilitate waveform and frequency analysis of vibrations below about $500 \mathrm{~Hz}$. This device can be used to detect amplitudes approximately ten times as great as is possible with photon-coupled interrupter modules [C. E. Gough, J. Acoust. Soc. Am. 75, 1770-1776 (1984); R. J. Hanson, Phys. Teacher 25, 165-166 (1987)]. The device uses a pair of synchronized self-scanning diode arrays mounted perpendicularly to each other such that each array responds to a shadow of the vibrating string. The output of each scan is two series of pulses. The pulses are converted to zeros and ones that are used to produce a pair of numbers which correspond to the absolute location in each coordinate component. The numbers are stored in the memory of the Apple II microcomputer in which the device is resident and are also routed to a pair of DAC's to regenerate the waveforms.

\section{0:05}

F5. Triggering algorithm for the Boie radio drum. Max Mathews (Music Department, Stanford University, Stanford, CA 94305-4060) and R. A. Boie (AT\&T Bell Laboratories, Murray Hill, NJ 07974)

The Boie radio drum is used to play electronic music synthesizers. The drum is a sensitive surface that tracks the position and velocity of two or more drum sticks in a shallow volume above the surface in three dimensions. Positions and velocities are determined by measuring the strength and distribution of electrical capacitance between the tips of the drum sticks and the surface. The drum is made force sensitive in the surface normal direction by coating either the sticks or surface with an elastic foam layer. Position information of a stick in contact then corresponds to two-dimensional position on the surface and force into the surface. One of the drums functions is to generate triggers that start notes. The trigger algorithm must be very reliable in that one and only one trigger is produced no matter how gently or how hard the drum is struck. An algorithm, patterned after the action of a piano key has been implemented. A trigger plane and a reset plane are defined above the drum surface. Motion through the trigger plane produces a trigger. Motion back through the higher reset plane enables the next trigger. Loudness is controlled by the stick velocity as it crosses the trigger plane. The algorithm gives very reliable triggers over a bigger dynamic range than existing synthesizer keyboards.

\section{$10: 20$}

F6. Chaotic dynamics of wood wind multiphonics. Douglas $H$. Keefe and Bernice Laden (Systematic Musicology Program, School of Music, DN10, University of Washington, Seattle, WA 98195)

Experimental evidence for chaos in multiphonic tones derives from measured power spectra, and more directly from the measured correlation dimension of the reconstructed phase space of the dynamical system. The frequencies $f_{l, m}$ of the line spectral components of a reed-driven woodwind multiphonic fit a biperiodic spectrum of low- to mid-playing levels; i.e., there exist base frequencies $f_{1}<f_{2}$ such that $f_{l, m}=l f_{1}+m f_{2}$, for non-negative integers $l$ and $m$. For an alto saxophone multiphonic, these base frequencies are phase locked, namely, their ratio is equal to a ratio of small integers. Using the same nominal saxophone fingering, this measured ratio equals $8: 5$ or $7: 4$ (within $0.1 \%$ ) depending upon player's adjustment of embouchure and playing level. A broadband spectrum is present in the saxophone multiphonic spectra at all but the lowest levels, which exceeds instrumentation noise and window leakage associated with signal processing. A phase-locked biperiodic spectrum superposed on a broadband background spectrum is characteristic of low-dimensional chaotic attractors in other fluid mechanical systems. At the highest playing levels, period doubling of the $f_{1}$ component occurs, the broadband level significantly increases, and phase locking is not observed. The dimension of the attractor is measured by embedding the time series in a higher space. The correlation dimension $D$ (Grassberger and Procaccia, 1983 ) is measured by embedding a single measured time series in a higherdimensional space, so to reconstruct the phase space of the dynamical system. For the saxophone multiphonic at the lowest and highest playing levels, $D=2.85 \pm 0.06$ and $2.84 \pm 0.12$, respectively. These fractal dimensions suggest that multiphonics are strange attractors. Data for other woodwinds will be presented.

\section{$10: 35$}

F7. Effect of pressure rise time on starting transients of flue pipes. A. W. Nolle (Department of Physics, University of Texas, Austin, TX 78712) and T. L. Finch (Department of Physics, St. Lawrence University, Canton, NY 13617)

A special wind supply provides adjustable rise time $T_{r}$. Adjustable closed and open pipes having frequencies 198 to $256 \mathrm{~Hz}$ are studied. The steady-state supply pressure is $530 \mathrm{~Pa}$. The precursor, at several times the fundamental frequency, and a subsequent burst dominated by the next mode above the fundamental are strongest for $T$, between 1 and 10 fundamental periods, but may vanish for larger or smaller $T_{r}$. For nearly harmonic pipe resonances, the fundamental and higher components can grow simultaneously, and the burst may exceed the steady-state amplitude. For more inharmonic modes (e.g., a wider pipe) the fundamental quenches the burst when the overall amplitude is well below steady state. External sound at the fundamental frequency suppresses both the precursor and the subsequent burst. Important features of the experimental results occur in a computer model that involves time-delayed nonlinear feedback and uses simple oscillators to represent resonator modes [N. H. Fletcher, Acustica 34, 224-233 (1976) ]. With numerical rather than analytical solutions it is possible to treat the realistic case of the fully switched jet. 
The development of culturally specific perceptual knowledge for mu-

F8. Perception of a musical construct by professional musicians. Philip Baczewski and Rosemary N. Killam (Computing Center, University of North Texas, P.O. Box 13495, Denton, TX 76203)

This study examined 15 highly trained listeners' response to a stimulus consisting of a 16-measure piece of music. Subjects were music performance graduate teaching fellows. They were presented with a taped performance (prepared by professional musicians) of three repetitions of a Mozart duet, together with the experiment's instructions. Experimental design allowed measurement of response to each stimulus repetition. Subject responses were recorded in one of two differing formats: eight subjects recorded the musical notation of each of the three stimulus repetitions in a different color of pen; seven subjects recorded the musical notation of each of the three stimulus repetitions on a different translucent paper overlay. Subjects were allowed $3 \mathrm{~min}$ to record each response. Data were encoded and analyzed to provide measurement of cumulative response arising from all three repetitions. Effects of pitch boundary and constituent groupings were obtained. A model emerges of complex perceptual strategies employed by musically sophisticated subjects. The strategies incorporate pitch relationships, contour, and grouping. [Work supported, in part, by an Apple Education Foundation Grant.]

\section{1:20}

F9. Vocal range for speech determines perception of a musical pattern. Diana Deutsch, Tom North, and Lee Ray (Department of Psychology, University of California, San Diego, La Jolla, CA 92093)

Previously, a two-tone musical pattern was described that possesses some highly unexpected properties [D. Deutsch, J. Acoust. Soc. Am. Suppl. $180, \mathrm{~S} 93$ (1986) ]. For any given listener, it is heard as ascending when played in one key, and yet as descending when played in a different key. As a further paradox, when the pattern is played in any key it is heard as ascending by some listeners but as descending by others. Since then, the behavior of this musical paradox has been explored under parametric variation [e.g., D. Deutsch, Percept. Psychophys. 42, 563-575 (1987)]; however, the basis for the individual differences remained elusive. Here, it is demonstrated that a striking correlate exists between the way this pattern is perceived and position of the listener's vocal range for speech. To the authors' knowledge, this is the first demonstration of a close connection between the perception of a musical pattern on the one hand and the listener's speech characteristics on the other. Implications of these findings for both music and speech are discussed. [Work supported by the Digital Equipment Corporation, and by the UCSD Biomedical Research Fund.]

\section{1:35}

F10. Native and non-native music perception by children. Michael P. Lynch (Departments of Pediatrics and Psychology, Mailman Center for Child Development, University of Miami, P.O. Box 016820, Miami, FL 33101) sic was investigated. Using a background/contrast paradigm originally developed for speech perception [Eilers et al., J. Speech Hear. Res. 20, 766-780 (1977)], Western 10-13 year olds and adults were tested in detection of mistunings in melodies based on native Western major and Western minor scales and a non-native Javanese pelog scale. Each age group was divided equally into musically inexperienced and musical subjects. The musically inexperienced adults had a perceptual bias for the melodies based on their native scales, but the adult musicians did not appear to share this bias, since they were able to generalize their knowl. edge of Western scales to the perception of a melody based on the Javanese scale. In contrast to the adults, the musician 10-13 year olds had a perceptual bias for the melodies based on Western scales, and the nonmusician 10-13 year olds did not share this bias. The musically inexperienced adults' performance was significantly better than that of the musically inexperienced children on both the Western and Javanese scales, and the same was true for the musician adults and children, indicating development of perceputal ability that is independent of musical training. Perception of Western mistunings was significantly related to the childrens' total years of musical experience and the amount of time devoted to practicing a musical instrument or voice. [Work supported by the American Psychological Association.]

\section{$11: 50$}

F11. The development of musical tuning perception from infancy to adulthood. Michael P. Lynch, Rebecca E. Eilers, D. Kimbrough Oller (Departments of Psychology and Pediatrics, Mailman Center for Child Development, University of Miami, P.O. Box 016820, Miami, FL 33101), and Paul Wilson (Department of Music Theory/Composition, School of Music, University of Miami, Coral Gables, FL 33143)

Three prominent hypotheses were evaluated by testing Western infants' and adults' abilities to detect mistunings in melodies based on native Western and non-native Javanese scales. The "innateness hypothesis" of speech perception applied to music perception suggests that infants are born with an equipotential to perceive the scales of any culture. The "naturalness hypothesis" of auditory perception suggests that the ear is best suited for processing the small-integer frequency ratios that comprise Western scales. Finally, the "perceptual loss hypothesis" of speech perception allied to music perception proposes that the ability to perceive non-native scales is reduced and the ability to perceive native scales is maintained from infancy to adulthood. In support of the innateness hypothesis and in contradiction of the naturalness hypothesis, 5- to 7 month-old infants did not possess a perceptual bias for either Western or Javanese scales. The perceptual loss hypothesis was weakened because, although adults possessed a perceptual bias for the Western over the Javanese scales, adults' perception of both these scale types was better than that of infants. [Work supported by the American Psychological Association.] 


\title{
Session G. Speech Communication I: Linguistics and Phonetics
}

\author{
Patti J. Price, Chairman \\ SRI International, EK178, Menlo Park, California 94025
}

\section{Contributed Papers}

\section{9:00}

G1. Prosody as a cue to syntactic structure: The role of prosodic information in disambiguating phonetically ambiguous sentences. Patti J. Price (SRI International, EK 178, Menlo Park, CA 94025), Mari Ostendorf (Boston University, Boston, MA 02215), Stefanie Shattuck-Hufnagel (MIT, Cambridge, MA 02139), and Cynthia Fong (Boston University, Boston, MA 02215)

To go beyond dictation tasks, speech recognition needs to be integrated with an understanding component. Natural language understanding research, however, has typically considered text input, and has not had to face the massive phonetic ambiguity arising from speech input: Even with no phonemic errors, word boundary ambiguities can yield thousands of parses for a substantial portion of moderate length sentences. This study assesses the potential role of prosody in aiding speech understanding. Using phonetically similar sentence pairs with and without a major structural break, seven types of structural ambiguity were investigated. Professional speakers read ten sentences of each type after reading diambiguating contexts. Naive listeners selected the appropriate context for each sentence. For recording and listening, only one member of each pair was used in each session; sentences were otherwise randomized. The results indicate substantial differences across structural types: e.g., appositions and tags were nearly always correctly identified, whereas main-main versus main-subordinate pairs were at the chance level. This suggests that prosody can disambiguate some, but not all, structural ambiguities. [Work supported by NSF.]

\section{9:12}

G2. Intonation and syntax: Preferred co-occurrences between pitch contour and sentence structure. Aart de Zitter and René Collier (Institute for Perception Research, P. O. Box 513, 5600 MB Eindhoven, The Netherlands)

Melodic models, also called "intonation grammars," specify the perceptually relevant properties of pitch movements and indicate which sequences of them constitute well-formed pitch contours [N. Willems, R. Collier, and Y. 't Hart, J. Acoust. Soc. Am. 84, 1250-1261 (1988)]. It appears that for each basic intonation pattern several contour variants can be generated that sound equally acceptable from a purely melodic point of view. However, not every contour is compatible with just any sentence. Indeed, in many cases the choices that speakers make appear to be far from random. Rather, they are governed by syntactic, semantic, and pragmatic considerations. The present paper concentrates on syntactic variables such as the degree of structural cohesion between pitch-accented words or the depth of the syntactic boundary between phrasal constituents. Experiments will be discussed in which syntactic variables appear to influence the preference of the speaker or the listener for particular pitch contours. It will be examined to what extent these results are relevant for improving synthetic intonation in text-to-speech systems.

\section{9:24}

G3. Phonetic evidence for a three-vowel system in Kabardian. JohnDongwook Choi (Department of Linguistics, UCLA, Los Angeles, CA 90024-1543)
The Kabardian vowel system has been the focus of much debate. Central to this debate is whether or not the low vowel a is quantitatively distinct from the mid vowel $\mathrm{a}$. This study examines phonetic evidence that bears on this issue. Spectrographic and durational analyses were conducted for 118 vowels taken from a Kabardian text read twice, once slowly and once at a normal rate, by three adult male speakers. Each vowel was measured for its first three formant frequencies and duration. The data reveal that the mean duration of $ə$ is $62.5 \%$ that of $a$, compared to the mean duration of $i$ which is $67.5 \%$ that of $a$. These figures represent ratios characteristic of intrinsic durational differences rather than distinctive length and support a three-vowel analysis. Qualitatively, the formant frequency measurements support the claims in the literature that Kabardian vowels contrast uniquely along the height parameter with some frontback allophony for the two higher vowels. Moreover, a has a mean $F 1$ of $690 \mathrm{~Hz}$ as compared to 2 which exhibits a mean $F 1$ of $510 \mathrm{~Hz}$, further supporting a three-vowel analysis. [Work supported by NSF.]

\section{9:36}

G4. Phonotactic constraints as a filter of the articulatory-acoustic signal. Maria-Josep Solé (Laboratori de Fonètica, Facultat de Lletres, Universitat Autònoma de Barcelona, 08193 Bellaterra, Barcelona, Spain)

The present study investigates the working and status of phonotactic constraints in the modeling of speech production and speech perception. Phonotactic constraints act as a filter of the acoustic signal and the subsequent articulatory gestures. The adaptation of foreign borrowings and foreign language learning provide many examples of the transfer of the phonotactic constraints of $L 1$ to the perception and production of $L 2$, e.g., English speakers' pronunciation ["nou"sei] for the Spanish sequence ["no"se]"I don't know," and [ $\varepsilon$ l $e i$ "trel" bel] for the French sequence [ $\varepsilon l \varepsilon$ "tre" bel] "she is very beautiful," shows how the English syllable structure constraint that prevents short vowels from occurring in stressed open syllables is imposed on these languages. When this syllable structure constraint is not present, though, as in the words [ $[$ l] "elle," [bel] "belle," English speakers pronounce a short vowel. To check whether higher-order phonotactic constraints act as a filter of the acoustic signal, the same physical signals were placed in different syllabic structures by digital manipulation with an ILS program. The stimuli were presented to speakers of different languages to check whether they perceived the same stimuli differently depending on the phonotactic constraints of their language. The results show that phonetic and phonological identification is overridden by phonotactic constraints, which advocates for a top-down processing of the signal.

\section{9:48}

G5. Finding and using stress accent in spoken language understanding. Shirley A. Steele and Suzanne A. Liebowitz (Unisys Corporation, P. O. Box 517, Paoli, PA 19301)

Stress accent is a feature of the prosodic system in English that functions syntagmatically to organize an utterance into shorter sections. Knowledge about the location of stress accents in the segmental stream can help a speaker-independent spoken language understanding system prune the search space, e.g., for the correct lexical item or the correct 
pragmatic interpretation. Unlike automatic systems, human listeners are able to locate accents because they know what accented versus unaccented syllables sound like and because they can normalize for such things as speaker and speaking rate. Here, the results of a study designed to locate accents in continuous speech are reported. An attempt is made to use both speech knowledge and normalization by identifying salient characteristics of accent in training data along with accent characteristics of the test utterance. Accented and unaccented syllables are separated using selected clustering algorithms on a feature space of energy and duration measures. In addition, some specific ways in which the output of the accent finder can be used by other components of the system (e.g., lexical access, pragmatics) are suggested. [Work partially supported by DARPA/ISTO.]

\section{0:00}

G6. More on domain-final lengthening and foot-level shortening in spoken English. Brad Rakerd, William Sennett, and Paul Cooke (Department of Audiology and Speech Sciences, Michigan State University, East Lansing, MI 48824)

The stressed syllables of spoken English tend to be lengthened when they occur before major syntactic boundaries (domain-final lengthening) and to be shortened when they are followed by unstressed syllables within the same metrical foot (foot-level shortening). The nature of the interaction between these two factors has been investigated. A first study [B. Rakerd, W. Sennett, and C. A. Fowler, Phonetica 44, 147-155 (1987)] suggested that their effects are independent. In that study, the syllabic structure of metrical feet spanning word boundaries were manipulated, where those boundaries either coincided with noun-phrase/verb-phrase boundaries or did not, and we compared stressed syllable durations. A limitation on the comparison was that the phonetic details of the metrical feet of interest were matched but not held constant across syntactic conditions. In the present study, the procedure to control phonetic details throughout has been refined. With this modification, a significant interaction was observed between domain-final lengthening and foot-level shortening; specifically, it was found that the intervals between lengthenings delimited the domains over which substantial shortening could occur. The whole pattern of results will be discussed in light of current theories of foot-level shortening.

\section{0:12}

G7. Updating UPSID. Ian Maddieson and Kristin Precoda (Phonetics Laboratory, Department of Linguistics, University of California, Los Angeles, CA 90024)

UPSID-the UCLA phonological segment inventory database - is a database containing the phoneme inventories of a large genetically based sample of languages [I. Maddieson, Patterns of Sounds (1984)]. Each phoneme is specified in terms of a comprehensive set of phonetic features. The first version of the database has proven useful to scholars interested in phonological universals and theories concerning the structure of phonological systems [e.g., B. Lindblom and I. Maddieson, in Language, Speech \& Mind, edited by L. M. Hyman and C. N. Li (1988); K. Stevens and S. J. Keyser, Language 65, 81-106 (1989) ]. An expanded and corrected secand version is currently in preparation. This version improves the sample, increasing coverage of previously undersampled language families and correcting a few oversampling errors, and correcting errors in individual language inventories. A new custom-written software package for MS-DOS systems provides economical and flexible means of storing and modifying this enhanced database and outputting subsets of the data for further analysis. The database is stored as several separate but interrelated modules. One contains a listing of character codes for each distinct segment type occurring in the database paired with a standard phonetic description and with the list of features assigned to that segment. Another contains the phoneme inventories as a set of character codes for each language. The database is principally used by mating information from these two modules, for example, by creating a file containing the fully specified feature descriptions of the segments in a group of languages, or all segments defined by a selected set of feature values. Such files can be exported to a standard statistics package for sophisticated processing, but simpler counting operations can be performed within the UPSID program.

\section{0:24}

G8. Accentedness and givenness: Change in word, syllable, or segment duration? Wieke Eefting and Sieb G. Nooteboom (Institute of Phonetics, University of Utrecht, Trans $10,3512 \mathrm{JK}$ Utrecht, The Netherlands )

In an earlier paper [W. Eefting, J. Acoust. Soc. Am. Suppl. 185, S97 (1989) ] the results of a study concerning the relation between the features "givenness" and "accentedness" and the duration of Dutch words were presented. Production data from a highly trained, professional speaker indicated that the duration of one syllable and three syllable words is lengthened when accented. Information value had only an effect on one syllable words: One syllable words containing new information were lengthened as compared to the same words containing old information. It remained unclear whether the differences in word duration affected the temporal structure of the whole word; a local change within the word might be responsible for the differences. In the present study, the durations of the syllables and the segments within the target words are measured. Data will be presented showing that all segments in the one syllable words and all syllables in the three syllable words contribute to the differences in word duration as described above. These findings support our claim that in speech the control of word duration is an active skill and that words are the most important units of speech communication. [Work supported by the SPIN-ASSP Program.]

\section{0:36}

G9. The syllabic attachment of post-vocalic liquids and nasals: Error elicitation evidence. Ann Stuart Laubstein (Department of Linguistics, Carleton University, Ottawa K IS 5B6, Canada)

Inferences based on spontaneous speech error corpora regarding the syllabic attachment of post-vocalic liquids and nasals are experimentally tested using the error elicitation technique described in Motley, Baars, and Camden (1983). In the first experiment, 44 subjects were tested on "Vr" and "Vl" sequences. As predicted $\mathrm{Vr}$ sequences were not split up, whereas V1 sequences were. Subjects moved the vowel significantly more often in Vl sequences than in Vr sequences (Wilcoxon, $p<0.001$ ); in addition "l" was moved significantly more often than " $\mathrm{r}$ " (Wilcoxon, $p$ $<0.0001)$. Here, $\mathrm{Vl}$ did, however, move as a unit, lending support to the Halle and Vergnaud ( 1980) sonorant constituent. The second experiment used 45 subjects to test the viability of such a constituent by comparing $\mathrm{Vl}$ to vowel nasal ("Vn") sequences, and nasal and liquid obstruent (SO) sequences to obstruent obstruent $(\mathrm{OO})$ sequences. As predicted $\mathrm{Vl}$ and $\mathrm{Vn}$ sequences were indistinguishable from each other and SO and OO were significantly different. This convergence of evidence from separate domains supports the generative assumption that constructs of competence underlie the capacity for use.

\section{0:48}

G10. Classification of Japanese syllables including the speech sound found in loan words. Shizuo Hiki (School of Human Sciences, Waseda University, Mikajima 2, Tokorozawa, 359 Japan)

Methods of classifying Japanese syllables are discussed with regard to their use in evaluating performance of speech processing techniques for both synthesis/recognition systems and user's speaking/listening charac- 
teristics. First, by taking into account the initial/intervocalic contrast of utterance, 16 intervocalic syllables are introduced to the traditional 50 Japanese syllables chart or the list of 100 Japanese syllables that has been used in articulation test of speech transmission channels. Then, by rearranging those syllables based on place/manner of articulation and by sup- plementing missing kind of combinations of the preceding consonant and following vowel, more than 60 new syllables which can be pronounced in the loan words are derived. Also discussed in this paper is rationale for various levels of deriving new syllables that correspond to the special Kana letters used in writing loan words in Japanese.

\author{
Session H. Engineering Acoustics I: Transducers, Materials, and Diffraction
}

\author{
Sung H. Ko, Cochairman \\ Naval Underwater Systems Center, New London, Connecticut 06320 \\ Stephen C. Thompson, Cochairman \\ Westinghouse Oceanic Division, Department 721, Cleveland, Ohio 44117
}

\title{
Contributed Papers
}

1:00

H1. Tapered-thickness piezoelectric ceramics: Models and characteristics. Peter G. Barthé and Paul J. Benkeser (School of Electrical Engineering, Georgia Institute of Technology, Atlanta, GA 30332)

Tapered-thickness piezoelectrics have been studied for use in novel devices and imaging techniques, such as wideband transducers and frequency-controlled beam translation. However, no adequate theoretical model exists. In this work, a staircase model is developed in which the thickness gradient is represented as discrete steps. Each step is modeled as a constant-thickness section, which contributes to the net response. This approach allows the results and techniques developed for conventional devices to be readily applied to those with tapered thickness; it emphasizes important similarities and differences, and provides physical insight into the dynamics of tapered piezoelectrics. The model shows that the distributed half-wavelength resonances dominate its response. In addition, however, a taper-dependent, transverse interaction exists among steps, which adds to the primary response. A coupled oscillator approach is used to model this transverse cross talk with an acoustic transmission line. The theoretical electric input impedance, spectral content, pulse, and continuous-wave pressure profile responses obtained with the model are shown to be in close agreement with the measured responses from several tapered piezoelectric transducers.

\section{1:15}

H2. Sound field calculation for a circular array transducer. Chankil Lee, Intaek Kim, and Paul J. Benkeser (School of Electrical Engineering, Georgia Institute of Technology, Atlanta, GA 30332)

Ultrasonic circular phased arrays have long been employed in sonar, NDT, and AGV applications. More recently, these arrays have been used in medical imaging applications. The ability of these arrays to image a $360^{\circ}$ field makes them ideal for intravascular imaging applications. However, intravascular applications often require the diameter of the array to be on the order of $1 \mathrm{~mm}$. The small size of the array dictates that trade-offs in its design, such as the number of elements versus resolution, be made. Field patterns produced by a circular phased array, required to evaluate such design trade-offs, have not been extensively examined. In this paper, a new field calculation method, which requires less computational time than existing methods for a given accuracy, is presented. The curved surface of the array, radiating either continuous-wave or pulsed signals, is subdivided into incremental areas which are small enough that the Fraunhofer approximation can be applied. The field pressure is computed by summing the contributions from all the incremental areas using the RayleighSommerfeld diffraction integral. Theoretical results obtained with this method are compared to those obtained using other methods as well as experimental data. The effects of some design parameters such as the geometry of the array, pulse bandwidth, and amplitude weighting of the excitation signal will also be presented.

\section{1:30}

H3. Computation of the performance of an electret microphone diaphragm. Peter L. Madaffari (Industrial Research Products, 321 Bond Street, Elk Grove, IL 60007)

An attempt was made to simulate the performance of a tensioneddiaphragm, electret-based, microphone. Use of commercial finite-element analysis programs were unsuccessful because of the computational system used. An alternate method was developed using a relaxation approach. A third-order equation for the balance of forces appeared to give approximate solutions for both tensioned and multiply supported diaphragms. Contours of physical deformation and acoustic compliance were generated and mapped. From these computations, an improved electret backplate and diaphragm system were designed.

\section{$1: 45$}

H4. Edge effects in standard shapes piezoelectric transducers-A new method for calculating elastic coefficients. Dov Hazony and Birol Ozkaya (Department of Electrical Engineering and Applied Physics, Case Western Reserve University, Cleveland, OH 44106) 
An electrical network similar to the Mason model is constructed to conform with the electroelastic properties of thin isotropic piezoclectric ceramics of standard shapes under the condition of short stress pulses in this thickness mode. It will be seen that these pulses travel close to the speed of sound in an unbounded medium and that it is possible to calculate the elastic coefficients by setting the two active faces free. Specifically, both $\lambda$ and $\mu$, the Lamé constants, may be calculated from one measurement.

\section{2:00}

H5. A high-resolution beamformer for acoustic imaging. Donald C. Knudsen (Knudsen Engineering Limited, Perth, Ontario, Canada)

This paper describes the development of a high-resolution imaging sonar system based on innovative digital beamforming technology. The new beamformer provides rapid access to a high-resolution, three-dimensional image field, scanning simultaneously in both azimuth and elevation, to produce a "staring" image as well as traditional range/bearing presentation. The new digital beamformer operates on sampled data, and uses time delays rather than phase shifts for broadband signal capability. It also features dynamic, pulse-tracking focusing from within one aperture of the array. An experimental imaging sonar with a 64-channel digital beamformer has been constructed. A highly parallel, pipelined architecture is employed to obtain a processing rate of four million beam output samples per second, delayed and summed across 64 hydrophone channels. This paper describes the technology and presents results obtained with the prototype system, including samples of imagery.

\section{2:15}

H6. A high-frequency omnidirectional source for a robot end effector. Jack Leifer and Ilene J. Busch-Vishniac (Department of Mechanical Engineering, The University of Texas at Austin, Austin, TX 78712)

A high-frequency omnidirection source has been designed that enables three-dimensional position monitoring in air to be performed. The source consists of a piezoelectric disk sandwiched between the large ends of two solid exponential Webster horns. When appropriately housed and driven to longitudinal resonance, the narrow end of one of the horns can be modeled as a baffled piston. Because of the high driving frequency ( 301 $\mathrm{kHz}$ ) and the small radius of the horn, the directivity pattern of the source contains no nodes. Preliminary data regarding the sound field produced by the source were presented previously [J. Leifer and I. J. Busch-Vishniac, J. Acoust. Soc. Am. Suppl. 1 85, S115 (1988) ]. Since that time, a more quantitative study of the source field has been performed, the results of which are presented here. It was found that the horns did not resonate exactly at the theoretically calculated frequency so a mechanical tuning process had to be employed. The predicted and achieved resonant frequency of the source differs by about 7\%. [Work supported in part by General Motors Research Laboratories.]

\section{2:30}

H7. Self- and mutual radiation resistance measurement by use of the sound intensity technique. Hideo Suzuki, Masazou Anzai, Takahiko Ono (Acoustics Laboratory, Ono Sokki Company Limited, 2-4-1 Nishishinjuku, Shinjuku-ku, Tokyo, 163 Japan), and Frank Fossaert (Institut Superieur D'electronique de Paris, Paris, France)

Radiated sound power from sources can be measured by use of the sound intensity technique. The radiated power is obtained by integrating the normal intensity over the surface surrounding a sound source. Since the total radiated power from sound sources is determined by self- and mutual radiation resistances, it is possible to determine the radiation resistances from the measurement of radiated power. For a sound reinforcement systern, it is useful to know the radiation resistances of loud speakers in a listening room. A knowledge of self- and mutual radiation resistances is also important for an active sound power or noise control. As an example, self- and mutual radiation resistances of two closely located loudspeakers measured in a lecture room are presented. Due to reflections, the resistance curves measured in a room are much more irregular compared to the resistance curve of a piston in an infinite baffle. The effect of the loudspeaker placement on the self and mutual resistances is very significant.

\section{$\mathbf{2 : 4 5}$}

H8. Bandwidth and absorption effects on intensification in a structuralacoustic enclosure. Linda F. Peretti and Earl H. Dowell (Department of Mechanical Engineering. Duke University, Durham, NC 27706)

Asymptotic modal analysis (AMA) provides a formal method to analyze high modal density sound fields in enclosures. Typically, uniform response levels occur throughout the interior. Exceptions occur at physical boundaries of the enclosure, and at theoretical boundaries that are created due to the symmetry of certain loading conditions. The transition from elevated response at a boundary to uniform interior response is studied as a function of the ratio of bandwidth to center frequency $\left(f_{b} / f_{c}\right)$, for combinations of 1-, 2-, and 3-D modes emanating from corners, edges, and walls (in the case of a rectangular enclosure). The size of the transition region scales with the center frequency and is affected by the bandwidth to center frequency ratio in varying degrees depending upon mode type and geometry. In the AMA limit, the intensification zone can be described by a local solution that is independent of the enclosure dimensions. Wall absorption affects the character of the intensification zone, and this effect is studied for various $f_{b} / f_{c}$ ratios. Local solutions formed from the superposition of oblique waves provide the formulation for intensification behavior. By expanding the result in a Taylor series about the center frequency, the intensification zone can be described by a set of universal curves that contain the center frequency effect and a bandwidth correction. [Work supported by NASA Langley Research Center.]

$$
\text { 3:00 }
$$

H9. Some calibrations and applications of the aerodynamic fan reference sound source. Angelo J. Campanella (Campanella Associates, 3201 Ridgewood Drive, Columbus, OH 43026)

Introduced by H. C. Hardy [Noise Control (May 1959)], the mechanical reference sound source (RSS) provides a broadband sound field by turbulent air shear at the perimeter of a rotating open centrifugal fan wheel. For a given wheel rpm, geometry, and air density, the emitted sound level is constant within less than $1 \mathrm{~dB}$ when averaged over a few seconds. The sound power is relatively uniform in octave or third-octave bands from 125 to $8000 \mathrm{~Hz}$. Usable levels of sound power are emitted above and below these frequencies, with field measurements in the $63-\mathrm{Hz}$ octave band feasible in large rooms. Small motor-driven units have endured for decades. Hemi-anechoic, reverberation room and intensity methods [R. Peppin et al., NOISE-CON 88, and W. D. Gallagher (private communication) ] for RSS calibration will be described. Low-frequency discrepancies between free-field and reverberation room calibration methods [P. Bruel, B \& K Review (1978, No. 3)] have diminished as the reverberation room method is improved (better sound diffusion, space averaging, and decay analysis) (P. K. Baade, 1989 ASHRAE Trans. (January 1989) ]. The portability and reliability of this device suit it especially to worldwide use in hostile environments such as factory assembly rooms and construction sites. Some recent experiences in application of the Acculab RSS to small and large device sound power measurement and room sound absorption will be described.

\section{3:15}

H10. Characterization of the properties of underwater acoustical materials. Robert Y. Ting (Underwater Sound Reference Detachment, U.S. Naval Research Laboratory, Orlando, FL 32856-8337) 
Increased emphasis on the development of new materials for underwater acoustical applications requires that material properties be carefully characterized during the research phase of the investigation. Once measured in the laboratory, the chemical, physical, and acoustical properties of the materials are correlated in order to enable one to design new acoustical materials. This approach has been successfully employed at NRLUSRD for the past years in the study of both new transduction materials and acoustical elastomers. Piezoelectric and dielectric properties of new ceramics and piezocomposites are characterized. Dynamic moduli in shear, tensile, and bulk modes are also determined for elastomers, while their chemical properties are analyzed by using a number of instrumental techniques, including gel permeation and liquid chromatography, Fourier transform infrared spectroscopy, and thermal gravimetric analysis. These measurement techniques will be described, and their frequency, temperature, and pressure capabilities will be discussed.

\section{$3: 30$}

H11. Diffraction about acoustically soft panels. Ronald P. Radlinski (Naval Underwater Systems Center, New London, CT 06320)

The use of two-dimensional shaded and unshaded arrays to discriminate against diffraction about acoustically soft panels is investigated numerically by the use of a two-dimensional boundary element integral method. The calculated predictions for an infinitely long strip are used to estimate diffraction at a hydrophone array behind a square baffle. The diffraction floor for insertion loss of hydrophone arrays is determined by weighting the hydrophone responses with various shading coefficients.
Comparisons of diffraction discrimination by a hydrophone array versus a single hydrophone at various positions are presented.

\section{$3: 45$}

H12. A generalized approach to random noise synthesis: Hardware implementation and performance characteristics. K. D. Hsueh and R. P. Hamernik (Auditory Research Laboratory, 107 Beaumont Hall, State University of New York, Plattsburgh, NY 12901)

A theoretical model and a computer simulation of a generalized approach to the synthesis of random noises were presented in an earlier paper [K. D. Hsueh and R. P. Hamernik, J. Acoust. Soc. Am. Suppl. 185, S40 (1989)]. In this paper, the hardware implementation for this new synthesis technique is described. The digitally generated analog signals transduced through a high-intensity acoustic driver show that manipulations in the phase domain can be advantageous in creating acoustic signals of widely varying descriptors (e.g., skewness, kurtosis, etc.) while maintaining identical amplitude spectra. In essence, entire families of random waveform having identical amplitude spectra but variable statistical properties can be created. The influence of the acoustic driver on the spectra and the statistical properties of the synthesized waveforms will be shown. Some examples of the flexibility of phase manipulations will be presented, e.g., (1) amplitude-modulated random phase and (2) convolutions of the synthesized waveforms with any arbitrary function. These results illustrate that a variety of phase manipulations can be performed to produce interesting families of complex random waveforms. The application of this methodology to acoustic or vibration testing, environmental stress screening, psychoacoustic studies, etc., will be discussed. [Work supported by NIOSH.] 


\title{
Session I. Psychological Acoustics II: Memorial Session for James P. Egan
}

\author{
Neal F. Viemeister, Cochairman \\ Department of Psychology, University of Minnesota, 75 East River Road, Minneapolis, Minnesota 55455 \\ Dennis McFadden, Cochairman \\ Department of Psychology, Mezes Hall, The University of Texas, Austin, Texas 78712
}

Chairman's Introduction-1:00

Contributed Papers

1:05

I1. Respect for the variables-James P. Egan remembered. James D. Miller (Central Institute for the Deaf, 818 S. Euclid, St. Louis, MO 63110)

In Egan's laboratory a variely of scientific eccentricities were forgivable or even desirable, but there was an absolute requirement that one "respect the variables." Respect for the variables included their careful measurement, accurate recording, their unconfounded control, and beyond that a certain reverence. Egan's precept of "respect for the variables" has had a special impact on hearing-aid research at CID. Since 1966 with the first attempts to understand why a simple microphoneamplifier-earphone combination performed better than a hearing aid [Miller and Neimoeller, J. Sound Hear. Res. (1967)] to the current work on fitting digital hearing aids, this work has been guided by the "respectthe-variables" precept. Methods developed at CID and elsewhere have led to increasing accuracy in specifying the acoustic performances of hearing aids in relation to the listener's auditory area. Progress and current issues will be reviewed.

\section{1:20}

12. Proportional target-tone duration as a limit on pattern discriminability: Multicomponent targets. Charles S. Watson and Gary R. Kidd (Department of Speech and Hearing Sciences, Indiana University, Bloomington, IN 47405)

Under high levels of stimulus uncertainty, pattern discrimination performance is often degraded so severely that it would be reasonable to question whether the same processing mechanisms used under minimaluncertainty conditions are still available to the listeners. It has previously been reported that the degrading effects of high stimulus uncertainty depend strongly on the proportional duration of a pattern component that is subject to change, relative to the total duration of the pattern [ $\mathrm{J}$. Acoust. Soc. Am. Suppl. 1 83, S40 (1987)], but only weakly on the absolute durations of the stimuli. Again using tonal sequences and a frequency discrimination task, this finding was extended to the case of multiplecomponent target sounds. Results suggest that detectability of pattern changes is a function of both the proportional duration of each changed tone and the number of changed tones, and not solely a function of the total proportion of the pattern that has been changed. [Work supported by AFOSR and NIH.]

1:35

13. Multiple looks and temporal integration. Neal F. Viemeister (Department of Psychology, University of Minnesota, Minneapolis, MN 55455) and Gregory H. Wakefield (Department of Electrical Engineering and Computer Science, University of Michigan, Ann Arbor, MI 48109)
Recent research on temporal resolution indicates that a person can listen using a temporal window with a time constant of less than $10 \mathrm{~ms}$. A basic question is how information from these brief "looks" is combined and processed. The initial focus is on the old problem of temporal integration and data are reported from an experiment similar to Zwislocki's twoclick experiment [J. Zwislocki, J. Acoust. Soc. Am. 32, 1046-1059 (1960) ]. The data are well described by assuming true integration for brief click separations and by assuming two independent looks for separations longer than approximately $10 \mathrm{~ms}$. Thus the data are more consistent with multiple looks than with true, long-duration temporal integration. To account for more general "integration" phenomena, a simple multiplelook strategy is compared with neural summation using spike data recorded from the auditory nerve of chinchilla for $C F$ tones. The decision statistic is either an optimally weighted combination of the spike count from contiguous short bins (looks) or the total spike count summed over the duration of the tone. The time-intensity trading functions (threshold for $d^{\prime}=I$ versus duration) for these schemes are very similar. This demonstrates, consistent with intuition, that a multiple-look scheme can show integration-like behavior and that such behavior does not imply true integration. [ Work supported by NS12125.]

\section{$1: 50$}

I4. Recognition memory for arbitrary, complex waveforms. William A. Yost (Parmly Hearing Institute, Loyola University, 6525 N. Sheridan Road, Chicago, IL 60626)

Recognition memory for arbitrary complex stimuli was measured using the operating characteristic technique developed by Egan [J. P. Egan, AFCRC TN 58-51, AD 152650 (1958) ]. Ten complex stimuli ( $500 \mathrm{~ms}$ in duration), each consisting of six, randomly chosen, equal amplitude sinusoidal components spanning frequencies from 300 to $3000 \mathrm{~Hz}$, were used as a training set. After 15 min of listening to the training set, listeners were presented a 20 -stimulus test set consisting of the ten training stimuli along with ten additional complex stimuli (generated in the same way as the ten training stimuli). In a recognition memory paradigm, lisleners used a five-point rating scale to rate their confidence that a stimulus presented from the test set was from the training set. Different constraints were placed on the selection of the complex waveforms to determine how those constraints affected recognition memory. For one condition there were no constraints except as described above; for a second condition the six-tonal components were harmonics (randomly chosen) of a common fundamental; and for a third condition the six-tone complex was sinusoidally amplicude modulated at rates of 4,16 , or $32 \mathrm{~Hz}$. The results will be discussed in terms of auditory object perception and the use of receiver operating techniques to measure recognition memory. [Work supported by a Program Project Grant from the NINCDS and a grant from AFOSR.] 
I5. The detection of in-phase and out-of-phase tones presented either randomly intermixed in the same blocks of trials or in separate blocks of trials. David S. Emmerich, William S. Brown, and James B. Thoms (Department of Psychology, State University of New York at Stony Brook, Stony Brook, NY 11794-2500)

When signals that are binaurally in phase are presented in a background of in-phase noise, they are generally considered to be detected by means of monaural processing. However, when out-of-phase signals are presented in the same background of in-phase noise, binaural processing is involved. One question addressed in the present research is whether subjects are able to process such monaural and binaural signals as efficiently when they are randomly intermixed within the same blocks of trials of a single-interval yes-no procedure, as when they are presented in separate blocks of trials. As in other experiments involving stimulus uncertainty, evidence was found of some impairment associated with listening for monaural and binaural signals simultaneously. Response latencies were also examined in order to gain additional information about stimulus processing. Latency-based operating characteristics were derived using the same procedure employed by Emmerich et al. [Percept. Psychophys. 11, 65-72 (1972) ], among others, and compared for monaural and binaural listening conditions.
I6. Spectral interference and the masking-level difference. Leslie R. Bernstein (Center for Neurological Sciences/Surgical Research Center University of Connecticut Health Center, Farmington, CT 06032)

Several investigations suggest that sensitivity to changes in interaural disparities within select spectral regions may be degraded by the presence of energy at other, even remote, spectral regions. It seemed interesting to determine whether similar degradations would be observed in an MLD paradigm. Detection thresholds were measured for NoSo and NoS $\pi$. The signal, and $800-\mathrm{Hz}$ tone $(100 \mathrm{~ms})$, was presented in continuous, broadband noise. Thresholds were also measured in the presence of an antiphasic, $400-\mathrm{Hz}$ tone (the interferer) gated simultaneously with the signal or presented continuously. NoS $\pi$ thresholds increased by about $7 \mathrm{~dB}$ with the gated interferer at $80 \mathrm{~dB}$ SPL. Smaller increases were observed with lower levels of the interferer. Slightly increasing or decreasing the frequency of the interferer away from the octave reduced substantially its effect, as did presenting it continuously. NoSo thresholds were affected only slightly by the interferer. Reversing the roles of the signal and interferer ( $400-\mathrm{Hz}$ signal, $800-\mathrm{Hz}$ interferer) led to smaller, but reliable degradations in performance. Homophasic interferers had, in general, smaller effects on performance. [Work supported by NIH and AFOSR.]
2:45

17. How onset, offset, and ongoing interaural delays affect lateralization. Thomas N. Buell and Constantine Trahiotis (Center for Neurological Sciences, Surgical Research Center and Department of Surgery (Otolaryngology), University of Connecticut Health Center, Farmington, CT 06032)

Several types of interaural delay can affect the lateral position of binaural signals. Delays can occur within the transient (onset and/off offset) or steady-state (ongoing cycle-by-cycle and/or envelope-based) portions of the signal, or both. The extent of laterality produced by these delays for simple and sinusoidally amplitude-modulated tones with an acoustic pointing task were measured. Relative potency of these delays was assessed by presenting them singly or in combinations (where the delays were consistent or in opposition). Also varied were rise/decay time, duration, rate of modulation, and center frequency. The major finding was that ongoing delays are much more potent than shown in previous tasks that required discriminations rather than judgments of location. [Research supported by NIH and AFOSR.]

18. Optimal stimulus placement in adaptive psychophysical procedures. David M. Green (Psychoacoustic Laboratory, Department of Psychology, University of Florida, Gainesville, FL 32611)

A common misconception is that the stimulus should be presented near the threshold value in an adaptive psychophysical procedure to minimize the variance of threshold estimates. For maximum likelihood procedures, recent work by Laming, Watson, and Pelli shows that the stimulus should be presented at a relatively high level. For some psychometric functions, they found the optimal stimulus placement level produces $84 \%$ to $94 \%$ correct in a two-alternative forced-choice task. This result is particularly disquieting, because the popular two-down one-up rule tracks a relatively low percentage of correct responses, $70.7 \%$. Our computer simulations, using a variety of psychometric functions, confirm the validity of their analysis. These simulations also demonstrate that the precise form of the psychometric function is not critical in achieving high efficiencies. Finally, data from human listeners indicate that the standard deviation of threshold estimates is approximately twice as large when the stimulus presented on each trial is at a stimulus level corresponding to $70.7 \%$, rather than $94 \%$ correct. [This research was supported by NSF and AFOSR.]

$$
\text { 3:15 }
$$

19. Psychoacoustical masking of one pure tone by another and its relation to a model of the mechanics of the basilar membrane. Julius $L$. Goldstein (Central Institute for the Deaf, $818 \mathrm{~S}$. Euclid Avenue, St. Louis, MO 63130)

Accelerated psychoacoustical masking effects with level for simple tone maskers of higher frequency tones [Wegel and Lane, Phys. Rev. 23, 226-285 (1924); Duifhuis, J. Acoust. Soc. Am. 67, 914-927 (1980) ] have clear correlates with nonlinear suppression and excitation phenomena in auditory nerve and basilar membrane responses (Abbas and Sachs, J. Acoust. Soc. Am. 59, 112-122 (1976); Patuzzi, Sellick, and Johnstone, Hear. Res. 13, 19-27 (1984); Robles et al., J. Acoust. Soc. Am. 80, 1364 1384 (1986) ]. A signal processing model of basilar membrane response is presented that quantifies basilar membrane mechanics as two nonlinearly interacting filter responses: (i) a sensitive, narrow-band compressive response responsible for the tips of cochlear suppression and excitation tuning curves; and (ii) and insensitive, lowpass linearlike response responsible for the low-frequency tails of tuning curves. Dual filter processing along with mutual suppression between the filters, described by the model, allows conceptualization of known psychoacoustics in terms of competing cochlear mechanical responses. Detailed model accounts of data and methods for estimating model parameters will be presented.

I10. A psychophysical evaluation of a model for suppression and excitation in remote masking. J. L. Goldstein, R. H. Gilkey, and R. E. Quiñónez (Signal Detection Laboratory, Central Institute for the Deaf, St. Louis, MO 63110) 
In the classical literature both the masking and the suppression of one tone by a second tone of lower frequency have been shown to be nonlinear functions of overall level. Here, the relation between these two tasks is further constrained by measuring both remote masking and suppression with the same subjects. The remote masking experiment measures the effect of a $602-\mathrm{Hz}$ sinusoidal masker on the detectability of a simultaneous 1500-Hz sinusoidal signal, while the suppression experiment measures the effect of a $602-\mathrm{Hz}$ sinusoidal suppressor on the forward masking produced by a $1500-\mathrm{Hz}$ sinusaid. The data agree with earlier studies and are interpreted in the context of the multiple band pass nonlinearity model [J. L. Goldstein, "Updating cochlear driven models of auditory perception: A model for nonlinear auditory frequency analyzing filters," in Working Models of Human Perception, edited by B. Elsendoorn and H. Bouma (Academic, New York, 1989), pp. 19-57], which suggests that both excitatory and suppressive mechanisms influence remote masking. [Work supported by NSF and AFOSR.]

$3: 45$

I11. Area under power-law receiver operating characteristics. Irwin Pollack (Mental Health Research Institute, University of Michigan, Ann Arbor, MI 48109-0720)

The receiver operating characteristic, or ROC curve, describes performance under a wide range of hit $(\mathrm{H})$ and false alarm (FA) probabilities. In actual practice, with many different experimental conditions, only a single hit-false alarm (H-FA) combination may be available to describe performance under each condition. One way to describe each performance level is in terms of the area under an ROC curve passed through the obtained H-FA combination. James Egan astutely observed that most ROC curves are well approximated by power-law functions of the form: $\mathbf{H}=[\mathrm{FA}]^{\boldsymbol{k}}$. Egan's power-law functions also satisfy the requirement of passing through the coordinate pairs: 0,$0 ; \mathrm{H}-\mathrm{FA}$; and $1.0,1.0$. The area under power-law ROC curves is simply $1 /(1+k)$. An alternative approach is to assume an ROC curve with linear segments between 0,0 and $\mathrm{H}, \mathrm{FA}$; and between $\mathrm{H}, \mathrm{FA}$ and 1.0, 1.0. The calculated area by the linear assumption is not constant for a fixed ROC curve; it varies with the H,FA level. The linear assumption underestimates, and at times seriously underestimates, the area under power-law ROC curves. The minimum error of underestimation is over $4 \%$ for $k$ between 0.05 and 0.5 . The maximum error of underestimation approaches the maximum possible error. Clearly, the linear approximation procedure can grossly distort the area under the ROC curve.

\section{4:00}

112. Spectral tuning for a temporal decline of masking. Dennis McFadden (Department of Psychology, University of Texas, Austin, TX 78712)

When a tonal signal of $1250-\mathrm{Hz}$ and 50 -ms duration is masked by a spectrally nonoverlapping, narrow-band noise centered at $2050 \mathrm{~Hz}$, the amount of masking observed depends upon the duration of this flanking noise band. Specifically, when the flanking band is gated on only for the duration of the observation interval (burst), detectability can be 20-25 dB worse for some subjects than when the flanking band is gated on a few hundred milliseconds prior to the observation interval (fringed). This temporal decline of masking as the fringe duration increases has the appearance of an adaptation effect-or what Egan used to call perstimulatory fatigue. When the fringe consists of a single tone of $2050 \mathrm{~Hz}$ preceding the flanking band, a substantial temporal decline of masking can still be obtained. As the frequency of this tonal fringe is varied around 2050 $\mathrm{Hz}$, the magnitude of the improvement relative to the burst condition diminishes, and, at least for most listeners, a "tuned" existence region can be mapped for this temporal deline of masking. Possible explanations include temporal development of the critical-band filter, and within-channel adaptation followed by across-channel masking. [ Work supported by NINCDS.]

\title{
Session J. Speech Communication II: Focus Session on Creaky Voice
}

\author{
Caroline G. Henton, Chairman \\ Linguistics Program. University of California, Davis, California 95616
}

Invited Papers

1:00

J1. Physiology of creaky voice and vocal fry. Ronald C. Scherer (Recording and Research Center, The Denver Center for the Performing Arts, Denver, CO 80204)

Creaky voice and vocal fry are voice qualities that are linguistically normal but often symptomatic in voice pathology. The physiology of creak and vocal fry will be discussed from the standpoints of electromyography, videofiberoscopy, $\mathrm{x}$-ray, glottography, and aerodynamics. Discussion will emphasize the relative role of the cricothyroid and thyroartenoid muscles, laryngeal vestibule conslriction, hyperadduction, vocal fold motion irregularity, subglottal pressures, mean flow, and glottal source flow. New data in these areas will be compared with earlier findings. [Work supported by the Bonfils Foundation.] 
J2. Acoustics of creaky voice. Ingo R. Titze (Voice Acoustics and Biomechanics Laboratory, Department of Speech Pathology and Audiology, The University of Iowa, Iowa City, IA 52242 and the Recording and Research Center, The Denver Center for the Performing Arts, Denver, CO 80204)

Creaky voice seems to be perceived as some combination of low pitch and roughness. Preliminary perceptual experiments suggest that whenever the fundamental frequency drops below about $70 \mathrm{~Hz}$, pulses are perceived in phonation. The combination of several closely spaced harmonics of different (and varying) amplitudes may then add roughness if the harmonics fall within the same critical band. In addition to relaxing the laryngeal muscles to produce low $F_{0}$, the larynx can create asymmetric vibrations and mode changes that produce subharmonic components. These contribute to the perception of low $F_{0}$. Subharmonics are a result of bifurcation of a single stable vibratory state into multiple quasistable states and can lead to chaotic vibratory behavior if asymmetries or nonlinearities in the system are increased.

J3. Sociophonetic aspects of creaky voice. Caroline G. Henton (Linguistics Program, University of California, Davis, CA 95616)

In a wide-reaching study of creaky voice in British English (Henton and Bladon, 1988) it was indicated that use of creaky voice varied significantly according to sex and accent of speakers, as well as with utterance position. Specifically, males used between three and ten times as much creaky voice as did females. Accentual differences were also found: Speakers of Modified Northern were more polarized in the overall amounts of creak used than were RP speakers. Individual speakers varied considerably in their use of creak, with a number of males being persistent creakers. In addition, creaky voice was shown to be an "end-of-utterance" phenomenon, used by females and males in a demarcative function. Further research using data from American English and from French indicates that creaky voice may be employed as a sociophonetic marker across cultures. Indeed, its role as a marker of male speech may be enhanced in certain cultures where gender-role expectations are more pronounced. The demarcative intonational function is observed again cross linguistically. This phenomenon, coupled with Hyman's (1988) recent results for West African languages, may make intonational creaky voice a candidate for a phonetic universal.

J4. Cross-linguistic relations between voice quality and vowel quality. Keith Denning (Department of English Language and Literature, Eastern Michigan University, Ypsilanti, MI 48197)

A survey of impressionistic and instrumental descriptions of diverse languages (including new data from the Nilotic languages Dinka and Acholi) reveals repeated cases of phonemic and subphonemic interaction between phonation type and aspects of oropharyngeal resonance. Generally, if there is a regular interaction between intra- and extralaryngeal factors in a language, vocal fold configurations with greater laryngeal laxness (as required for regular phonation in consonants, murmur in consonants and vowels, and pitch depression) are associated with lower $F 1$ and, conversely, greater vocal fold tension (associated with voicelessness, creaky phonation, and higher pitch) occurs with higher $F 1$. Explanations for this universal in biomechanical (Laver, 1980), neuromuscular (Laufer and Condax, 1981), and aerodynamic (Ohala, 1983) aspects of articulation are proposed. Cross-linguistic evidence suggests that articulation from intra- and extralaryngeal domains (putatively separate for phonological purposes) may function distinctively only in combination with each other in many cases. Implications of these and other findings include the need for a distinctive feature of "complex voice quality," in contradistinction to the "primary feature" approach of Stevens, Keyser, and Kawasaki (1986), and the desirability of phonological models that accommodate phonetically based patterns of the relevant type in formulating principles of sound change and synchronic phonological analysis. 


\title{
Session K. Physical Acoustics II and Bioresponse to Vibration I: Biophysical Mechanisms as Bases of Safety Criteria for Medical Ultrasound I
}

\author{
Wesley L. Nyborg, Chairman \\ Department of Physics, University of Vermont, Burlington, Vermont 05405
}

Invited Papers

1:15

K1. Chairman's introduction: Mechanisms as bases for safety criteria. Wesley L. Nyborg (Physics Department, University of Vermont, Burlington, VT 05405)

There is now considerable interest around the world in developing scientifically based safety limits for medical ultrasound, especially as applied in diagnosis. While there has been no evidence of harm caused by past exposures to diagnostic ultrasound, some of the newer applications employ higher intensities than were used formerly. Hence, there is need for quantitative information on acoustic conditions that pose different degrees of risk, to guide manufacturers, users, and regulators. It is fairly generally agreed that a promising way to generate such information is to consider the separate biophysical mechanisms by which ultrasound can cause biological damage. This special session is to deal with research findings that relate to these mechanisms, and their relevance to safety limits.

1:30

K2. Nonlinear aspects of ultrasonic heating. E. L. Carstensen, D. Dalecki, K. J. Parker (Department of Electrical Engineering, Biophysics, and Radiology, and the Rochester Center for Biomedical Ultrasound, University of Rochester, Rochester, NY 14627), R. Bacon (Division of Radiation Science and Acoustics, National Physical Laboratory, Teddington, Middlesex TW 11 OLW, United Kingdom), and D. Blackstock (Department of Electrical Engineering, Unversity of Rochester, Rochester, NY 14627 and Department of Mechanical Engineering and Applied Research Laboratory, University of Texas at Austin, Austin, TX 78712)

Under appropriate conditions, the absorption of finite amplitude ultrasound is determined largely by source amplitude, field geometry, and the nonlinear properties of the medium and is only weakly dependent upon the small signal absorption coefficient of the material. The characteristics of this absorption are completely foreign to the ideas that we have come to associate with small signal losses. Under certain conditions, finite amplitude absorption is somewhat greater in locused than in plane-wave fields. Under medically relevant conditions, heating of tissues may be enhanced by a factor of 3 or more by nonlinear phenomena. Some commercially available diagnostic instruments may be capable of producing focal pressure amplitudes high enough to result in nonlinear contributions to local heating. The resulting total temperature increments could exceed the $1{ }^{\circ} \mathrm{C}$, the value universally regarded as safe. Finite amplitude processes affect the thresholds for tissue damage. The characteristics of the threshold curves differ qualitatively depending upon whether they are expressed in terms of local or source intensities. [This work was supported in part by U.S.P.H.S. Grant No. CA39241.]

\section{$1: 55$}

K3. Biophysical characterization of diagnostic ultrasound equipment. Gail ter Haar (Physics Department, F Block, Institute of Cancer Research, Sutton, Surrey, United Kingdom) and Francis Duck (Medical Physics Department, Royal United Hospital, Bath, Avon, United Kingdom)

Concern for the safety of diagnostic ultrasound procedures has meant that there has been a move to classify ultrasonic scanning equipment in terms of the physical effects it can produce in biological tissues. To this end, different types of diagnostic equipment currently in use in the clinic have been characterized in terms of their heating effects, their ability to induce cavitation bubbles in agar gels, the streaming velocity attained under freefield conditions in water, and their ability to produce sonoluminescence. The occurrence and extent of these phenomena have then been studied in the light of the acoustic parameters (such as peak positive pressure, peak negative pressure, spatial peak temporal average intensity, etc.) used to produce them. Extensive characterization of the machines investigated was carried out using a beam calibrator. It was found that none of the acoustic parameters measured provided an unambiguous indication of the extent to which any of these physical mechanisms would occur, thus rendering it difficult to predict safety on this basis from a listing of acoustic output. 
K4. In vitro ultrasonic heating of fetal bone. J. L. Drewniak, K. I. Carnes, and F. Dunn (Bioacoustics Research Laboratory, University of Illinois, 1406 West Green Street, Urbana, IL 61801)

Estimates of temperature increase in soft fetal tissues exposed to medical ultrasound can be made with existing knowledge. However, little knowledge is available to make a resonable estimate of the temperature increase in fetal bone and surrounding tissues during in vivo exposures. An experimental study of the temperature increase measured in vitro in human fetal femurs exposed to $1-\mathrm{MHz}$, continuous wave ultrasound at $37^{\circ} \mathrm{C}$ has been conducted. The temperature increase in the femur specimens is measured with a small implanted thermocouple probe. Specimens range in gestational age from 59 to 150 days. The temperature increase measured in a 59-day gestational age specimen at $20 \mathrm{~s}$ following the initiation of exposure at $1 \mathrm{~W} / \mathrm{cm}^{2}$, for example, was $0.10^{\circ} \mathrm{C}$, while the temperature increase for a 108-day specimen with the same exposure conditions was $2.9^{\circ} \mathrm{C}$. A temperature increase of $4.0^{\circ} \mathrm{C}$ was measured in the 108-day specimen for a $60 \mathrm{~s}$ exposure at $1 \mathrm{~W} / \mathrm{cm}^{2}$. The temperature rise is found to increase linearly with intensity over the range of 0.5 to $10 \mathrm{~W} / \mathrm{cm}^{2}$. The initial rate of the temperature increase, evaluated at $0.2 \mathrm{~s}$ following initiation of exposure, has also been measured and is found to increase rapidly with gestational age. For example, $I^{-1} d T / d t$ at $0.2 \mathrm{~s}$ measured for the 59-day specimen is $0.06{ }^{\circ} \mathrm{C} / \mathrm{s} /\left(\mathrm{W} / \mathrm{cm}^{2}\right)$, while for the 108 -day specimen $0.81{ }^{\circ} \mathrm{C} / \mathrm{s} /\left(\mathrm{W} / \mathrm{cm}^{2}\right)$ was measured. The values of initial temperature increase measured in the fetal femur specimens can be compared to that obtained for soft tissue using the quantity $I^{-1} d T / d t=2 \alpha / \rho C_{p}$. For $\alpha=0.05 \mathrm{~cm}^{-1}$, and $\rho C_{p}=3.78 \mathrm{~J} /{ }^{\circ} \mathrm{C} / \mathrm{cm}^{3}$, $I^{-1} d T / d t=0.026^{\circ} \mathrm{C} / \mathrm{s} /\left(\mathrm{W} / \mathrm{cm}^{2}\right)$. The values of $I^{-1} d T / d t$ measured for the 59 - and 108 -day gestational age specimens are approximately 2 and 30 times greater than that for soft tissue.

\section{2:45-3:00 \\ Break}

K5. The potential role of acoustic cavitation in medial ultrasound bioeffects. L. A. Crum, R. A. Roy, and C. C. Church (National Center of Physical Acoustics, University of Mississippi, University, MS 38677)

Ultrasound is used in a variety of modalities in medicine. Some of these uses are: therapeutic-in which ultrasound is intended to have a (beneficial) biological effect; mechanical-in which vibrating tools are used as surgical scalpels, as dental scalers, etc.; diagnostic-in which clinical evaluations are made of internal organs and body conditions; clinical-in which high-intensity shock waves are used to comminute undesirable concretions such as renal or biliary calculi. For all these modalities, when their waveforms and typical acoustic intensities are reproduced in aqueous liquids in the laboratory, acoustic cavitation normally occurs. Since cavitations can also result in severe mechanical damage to objects in its vicinity, its presence in vivo is therefore to be avoided. This group has been active for some years in the study of cavitation produced by medical ultrasound devices and reports will be presented on various aspects of these investigations. [Work supported in part by NIH Grant No. 2 R01CA 39374-04 A1, and by the Office of Naval Research.]

K6. Acoustic scattering from transient, micron-sized cavitation bubbles. Robert E. Apfel, Sameer Madenshetty, Ronald A. Roy, and Qihong Xu (Department of Mechanical Engineering, Yale University, New Haven, CT 06520)

If cavitation occurs in vivo due to the application of medical ultrasound, it is likely to be difficult to observe. Concern rests less with the destruction of biological cells and more with the more subtle subcellular injuries that could result in damage to components responsible for cell reproduction. Cavitation on the scale of single biological cells-microcavitation-can be observed in vitro by scattering high-frequency ultrasound ( 30 $\mathrm{MHz}$ ) off of single transient cavitation bubbles. An active cavitation detection (ACD) has been used to determine the onset conditions (e.g., cavitation thresholds) in vitro for water with various artificial cavitation nuclei. More recently, the same scattering technique has been employed to observe the radius versus time characteristics of transient cavitation bubbles of one or more cycles. Reports on both threshold measurements and attempts to measure quantitative radius versus time histories of individual bubbles will be presented. The results will be presented in the general context of the joint Yale-National Center for Physical Acoustics collaborative effort to assess the safety of diagnostic ultrasound. [Work supported by the National Institutes of Health through Grant 4R01-CA39374.]

\section{3:50}

K7. Implications of gas body activation for medical ultrasonics. Douglas L. Miller (Battelle Pacific Northwest Laboratories, P.O. Box 999, Richland, WA 99352)

A stabilized body of gas in a biological medium can be directly activated (set into oscillation) by megahertz-frequency ultrasound, and cause nonthermal bioeffects at levels below the cavitation threshold. This phenomenon has been studied in plant tissue with intercellular gas-filled channels, in insect tissue with gasfilled tracheal systems, and in cell suspensions provided with gas trapped in hydrophobic micropores. The highly localized oscillation leads to the gathering of particles by radiation forces and to damaging shear stresses from acoustic microstreaming flow. In tissues, the bioeffects seem to have discernible thresholds and to depend on the temporal-peak intensity of exposure. In cell suspensions, for which effects involve collection of cells, a 
dependence on the square of the temporal-average intensity is discernible. In these model systems, significant effects can be observed with exposure parameters similar to those encountered in diagnostic medical applications. Furthermore, studies of decompression sickness and cavitation indicate that stable gas bodies may sometimes exist in mammalian tissue. Although further study of possible hazards is clearly needed, the existence of even this potential risk may justify concern about frivolous exposures with medical devices. [Work supported by PHS grant CA42947 awarded by the National Institutes of Health.]

\section{Contributed Papers}

4:15

K8. Ultrasonic heating of the skull. Edwin L. Carstensen, Sally Z. Child (Department of Electrical Engineering and The Rochester Center for Biomedical Ultrasound, The University of Rochester, Rochester, NY 14627), Stephanic Norton (The Rochester Center for Biomedical Ultrasound, The University of Rochester, Rochester, NY 14627), and Wesley Nyborg (The Rochester Center for Biomedical Ultrasound, The University of Rochester, Rochester, NY 14627 and Physics Department, University of Vermont, Burlington, VT 05405)

Comparatively simple analysis shows that diagnostic ultrasound devices, in some cases, may approach output levels that can produce significant heating of tissues, particularly if the propagation path includes bone. The model assumes that all of the ultrasonic energy transmitted into the bone is converted into heat at the surface. The heat source, therefore, is a disk with diameter equal to the sound beam. Experimental tests of these predictions using rodents show that temperature increments of the order of $3{ }^{\circ} \mathrm{C} / \mathrm{W} / \mathrm{cm}^{2}$ are produced in skull bone with sharply focused fields under medically relevant conditions. [This work was supported in part by U.S.P.H.S. Grant No. CA 39241.]

K9. Temperature rise in a focused Gaussian beam of ultrasound. Junru Wu and Gonghuan Du (Department of Physics, University of Vermont, Burlington, VT 05405)

The steady-state temperature elevation generated by a focused Gaussian beam including the effect of perfusion has been calculated along the beam axis. The medium is assumed to be a homogeneous absorbing one. The results indicate: (1) the temperature rise is an increasing function of the intensity gain of the focusing transducer, but seems never to exceed twice of that at the interface of the transducer and the medium generated by its unfocused counterpart; (2) the temperature rise at the interface of the transducer and the medium is not affected by focusing significantly. [Work supported by the American Cancer Society (IN-156) and by NSF and Vermont Epscor.]

4:45

K10. Bubble penetration-A major mechanism causing cell lysis? A. R. Williams (Department of Medical Biophysics, University of Manchester Medical School, Manchester M13 9PT, England)
The dramatic increase in the amount of cell lysis observed when the exposure chamber is rotated has been shown to be the result of recycling cavitation bubbles through the suspension [D. L. Miller and A. R. Williams, Ultrasound Med. Biol. (in press)]. In addition, the destructive effects of these bubbles have been shown to be reduced when the number of cells within the suspension was increased, with fixed cells being much more inhibitory than fresh cells [Ellwart et al., Ultrasound Med. Hiol. 14, 43-50 (1988) ]. It is proposed that under these exposure conditions cell lysis is caused by collision between cells and rapidly moving gas bubbles. If the bubble velocity is high enough then the cell is penetrated and lysed, but this interaction also slows down the bubble. Below a certain critical velocity it is presumed that the bubble attaches to (or is trapped within) a cell and ceases to interact with other cells. Additional support for this proposed mechanism has been obtained from studies utilizing erythrocytes embedded in agar gels and from histological observations in vivo [C. J. Martin et al., Ultrasound Med. Biol. 7, 235-265 (1981)].

K11. The study of cavitation in tissuelike gels. A. J. Mortimer, B. J. Trollope, and E. Birnbaum (National Research Council of Canada, Ottawa, Ontario K1A 0R6, Canada)

A study of the effects of cavitation in agar gels was undertaken as an extension of studies in this laboratory of cavitation-induced free radicals in aqueous media using the free radical scavenger terphthalic acid. Agarose gels were made at a concentration of $1.5 \%$ agarose and $1 \%$ terphthalic acid in distilled, deionized water. Gels were exposed to ultrasound for times of up to $3.5 \mathrm{~min}$ in both pulsed $(1: 1,100-1000 \mathrm{~ms})$ and continuous modes, at intensities from $1-2 \mathrm{~W} / \mathrm{cm}^{2}$. The liquid was then separated from the gel by dialysis and the fluorescence counted using a spectrofluorimeter. In all cases no fluorescence was measured; however, at the higher intensities, visible bubbles could be observed after treatment. At the same intensities and exposure conditions, significant levels of free radical production were observed in aqueous solution and in gels made from previously irradiated terphthalic acid. These results indicate that either cavitation thresholds in aqueous gels are significantly higher than would be expected from studies in aqueous solutions, or that the medium acts as an effective free radical scavenger, thereby limiting the possible biological effect of acoustic cavitation. 


\author{
Session L. Noise I: Aircraft Noise \\ Gilles A. Daigle, Chairman \\ Division of Physics, National Research Council, Montreal Road, Ottawa, Ontario K1A OR6, Canada \\ Chairman's Introduction-1:30 \\ Invited Papers
}

L1. Sonic boom measurements from very high altitude and Mach numbers on space shuttle-orbiter reentry. $J$. F. Stanley, E. G. Stansbery (NASA-Johnson Space Center, Houston, TX 77058), H. R. Henderson, and D. J. Maglieri (Eagle Engineering, Hampton, VA 23666)

STS-orbiter reentry sonic boom measurements are presented with particular emphasis on those acquired at very high altitude and Mach numbers. Included, also, are the orbiter physical characteristics and reentry parameters along with a discussion of the nature of its sonic-boom characteristics as compared to previous experience with aircraft. The applicability of existing sonic-boom theory, used for aircraft, to the STS-orbiter is shown. Previous STS reentry boom measurements, along with recently acquired measurements from the STS26 and STS-27 reentry are presented and discussed. The results indicate that there are no significant differences in sonic-boom signature characteristics from STS-orbiter and other aircraft. $N$-wave type signatures are observed on STS-orbiter up to Mach 23 and altitude of $250000 \mathrm{ft}$ having an overpressure of about $0.1 \mathrm{psf}$ and a period of about $3.0 \mathrm{~s}$. It is also shown that orbiter reentry maneuvering conditions do not produce focus booms. Although STS-orbiter signatures are predictable up to Mach 6 and an altitude of $130000 \mathrm{ft}$, sonic-boom theory improvements are required for Mach numbers greater than 6.0. [Work supported by NASA and USAF.]

L2. Weaker sonic booms may be considerably more quiet. Allan D. Pierce (Graduate Program in Acoustics, Pennsylvania State University, 157 Hammond Building, State College, PA 16804)

Present indications are that realistic designs of civilian supersonic aircraft can be achieved so that the sonic boom at the ground has substantially smaller pressure jumps (shocks) than was the case for previous designs and for the Concorde. Unknown as yet is the extent to which this achievement may increase public acceptability to the intrusion of occasional sonic boom events. The present paper reviews physical acoustics research that may pertain to this question, and examines the magnitude and possible definitions of rise times of sonic boom shocks. The detailed pressure profile at a shock determines the high-frequency content of the boom and affects the perceived loudness and the startle effect, with the rise time being a rough descriptor of this high-frequency content. Earlier theoretical considerations had suggested that the rise time, on the average, should be inversely proportional to the shock jump. Molecular relaxation effects, however, are now known to play a major role and lead to the intriguing prediction that rise times for shock jumps below approximately 50 Pa are substantially longer than one would extrapolate from measured rise times of stronger shocks using the simple inverse proportionality relation. The extent and nature of atmospheric turbulence effects are still incompletely understood and stimulate further research. [Work supported by NASA-LRC and by the William E. Leonhard endowment to Pennsylvania State University.]

\title{
2.35
}

L3. Noise from low-altitude military flying. Kenneth J. Plotkin (Wyle Laboratories, 2001 Jefferson Davis Highway, Suite 701, Arlington, VA 22202) and John A. Molino (Tech U-Fit Corporation, 400 Madison Street, \#210, Alexandria, VA 22314)

Military air tactics rely on increasing use of high-speed low-altitude penetration techniques. Training for this type of operation can take place in specially designated low-altitude military operation areas (MOAs) or along military training routes (MTRs). Environmental assessment of noise from these operations has become an important factor in the design of these special use airspaces. The noise environment is different from commonly studied situations such as airport or highway noise in urban/suburban areas, and it is not obvious that the usual $L_{\mathrm{dn}}$ analysis is appropriate. Individual events are loud (maximum levels in the 100-115 dBA range) and short ( $10 \mathrm{~dB}$ down durations of a few seconds). Noise events are infrequent (typically less than 3 or 4 per day on busy routes) and occur at random times. These operations also take place in quiet rural areas. A discussion is presented of the nature of these operations and the resultant noise environment. This noise is 
contrasted with well-understood noise environments. An interim noise metric, $L_{\mathrm{dnmr}}$ (monthly average, onset rate corrected $L_{\mathrm{dn}}$ ), developed for the U.S. Air Force is presented. A comprehensive research plan to test $L_{\text {drmr }}$, or replace it with a more appropriate metric, is discussed.

3:05-3:20

Break

\section{Contributed Papers}

\section{$3: 20$}

L4. Voyager flight crew hearing threshold levels resulting from 5- and 9day continuous in-flight noise exposure. Mark $R$. Stephenson (Biological Acoustics Branch, Armstrong Aerospace Medical Research Laboratory, Wright-Patterson AFB, OH 45433-6573), Bradley L. Billings (Audiology Center of Redlands, 245 Terracine Boulevard, Suite 101, Redlands, CA 92373), and George A. Jutila (Fortuna Family Medical Group, 874 Main Street, Fortuna, CA 95540)

The flight crew of the Voyager aircraft was continuously exposed to a broadband noise for nearly 5 days during a trial flight, and for over 9 days during their nonstop flight around the world. Evaluation of the threshold shifts resulting from these exposures represents a unique opportunity to study the effect of human exposure to intense continuous noise for such durations. Post-flight audiometry demonstrated that the 9-day fight did not result in larger hearing threshold shifts than those following the 5-day flight. Neither crewmember incurred a permanent threshold shift from these exposures.

\section{3:35}

L5. Law-frequency noise for low flying aircraft. Louis C. Sutherland (Wyle Laboratories, 128 Maryland Street, El Segundo, CA 90245), Kenneth Plotkin (Wyle Laboratories, 2001 Jefferson Davis Hwy., Arlington, VA 22202), and Jerry Speakman (Department of the Air Force, AAMRL (AFSC), Wright-Patterson AFB, OH 45433)

A consideration in the evaluation of aircraft noise impact on structures near airports and under low-level military training routes is the significance of the low-frequency content below $50 \mathrm{~Hz}$. Based on analysis of measurements obtained under military training operations, a preliminary prediction model has been developed for application to environmental assessment of possible effects on structure. The madel is based on the hypothesis that the major source to this low-frequency noise is radiation from the aircraft wake and vortices shed by the airflow over the wings or fuselage. The resulting empirical model predicts that the average maximum one-third octave band noise level, $L$, in decibels, in the frequency range of 5 to $40 \mathrm{~Hz}$ is given by: $L=48 \log [V / 100]+10 \log [A /$ $\left.R^{2}\right]+91$, where $V$ is the aircraft speed in $\mathrm{kn}, A$ is the wing area, and $R$ is the slant range. Fortuitously, the same expression also provides reasonable estimates of this low-frequency noise level in the same frequency range for helicopters where $V$ is now the helical tip speed and $A$ is the rotor disk area. The resulting empirical model is similar to that developed for higher frequency airframe noise (J. C. Hardin, NASA SP-416, 1976). Further study is required to assess the validity of this model in comparison to more sophisticated models of low-frequency airframe noise. Comparisons between the prediction model and measured data will be illustrated. [Work supported in part by U. S. Air Force Harry G. Armstrong Aerospace Medical Research Laboratory.]

\section{3:50}

L6. Loudness of sonic boom waveforms with atmosphere-induced rounding. R. A. Sohn and D. W. Schowe (Douglas Aircraft Company, M/C C1-E41 (212-20), 3855 Lakewood Boulevard, Long Beach, CA 90846)
The effect of atmosphere-induced rounding on the loudness of sonic boom waveforms is investigated. Waveforms with varying degrees of roundness representative of measured flight test data are considered. Simple equations are developed by which the perceived loudness and Cweighted sound exposure levels of these sonic booms can be calculated directly from their waveform parameters. This method shows excellent agreement with more complex loudness calculations (e.g., by Johnson and Robinson) as well as the full $\mathrm{C}$-weighted sound exposure level calculation. A new set of flight test data is used to correlate shock strength with rise time. The results are compared to a semi-empirical model proposed by Dr. Kenneth Plotkin from earlier work.

\section{4:05}

L7. Fluctuating pressure and thermal environments of runway joint seals exposed to jet engine exhaust. Timothy $W$. Lancey (Department of Mechanical Engineering, California State University-Fullerton, Fullerton, CA 92634) and Eugene E. Cooper (Naval Civil Engineering Laboratory, Port Hueneme, CA 93041)

Jet engine exhaust flow impinging on runway joint seals creates an aerothermoacoustic environment consisting of fluctuating pressure (acoustical and hydrodynamic) and incident gas temperature rapidly increasing with time. Fluctuating pressure at the surface of a joint seal on a runway was measured with microphones and low-pressure transducers positioned along the runway centerline. Temperatures were measured with thermocouples imbedded within the seal and on the surface, along the runway centerline and at several sideline distances. Pressure and temperature data were recorded during aircraft takeoff and landing operations. Measurements indicated incident noise pressure levels of approximately $164 \mathrm{~dB}$ re: $20 \mu \mathrm{Pa}$, surface temperatures over $155^{\circ} \mathrm{C}$, and seal internal temperatures of $100^{\circ} \mathrm{C}$. Fluctuating velocity of the seal was calculated, treating the seal as a fluid fowing between parallel walls. Energy absorption rate was found to equal $850 \mathrm{~W} / \mathrm{m}^{2}, 4.7 \%$ of the power available from the incident fluctuating pressure field. Thermal energy through convective heat transfer was absorbed at $34000 \mathrm{~W} / \mathrm{m}^{2}, 97.5 \%$ of total energy absorption.

\section{$4: 20$}

L8. Environmental impulse sound evaluation by $L_{\mathrm{Dl}}$ index: Physical and subjective tests. G. Brambilla, G. B. Cannelli (Istituto di Acustica, CNR, Via Cassia 1216, 00189 Roma, Italy), and S. Santoboni (Istituto di Tecnologia, Università Reggio Calabria, Via Diana 4, 89100 Reggio Calabria, Italy)

Direct measurements of the $L_{\mathrm{D} 1}$ index were performed by a new digital apparatus [G. B. Cannelli and S. Santoboni, Italian Patent No. 48694 A/87 and International Patent in progress] on a group of sounds having different impulsivity and recorded in the environment. The measurements showed that the $L_{\mathrm{D} 1}$ index accounts for fast fuctuations of sound level better than other noise indexes can do. In addition, the results indicated that the time constant is a critical parameter in measuring the impulse component of a sound. The sound recordings were presented in the laboratory at different sound levels and in balanced order to a group of naive subjects. They were asked to rate annoyance and impulsivity of each 
sound stimulus using a ten-point numerical scale [G. Brambilla, M. R. Carretti, and S. Santoboni, Proc. Noise 88, Vol. 3, p. 159, Stockholm (1988) ]. Correlation between the $L_{\mathrm{DI}}$ values and the subjective ratings is in progress in order to test the validity of this index in the evaluation of both impulsivity and annoyance of noise. [Work partially supported by CEC.]

\section{$4: 35$}

L9. A general analysis of the active control of sound radiation from a baffled panel. Scott D. Snyder, Pan Jie, and Colin H. Hansen (Department of Mechanical Engineering, University of Adelaide, GPO Box 498, Adelaide, South Australia 5001, Australia)
Previous theoretical investigations of active means to control sound radiation from a vibrating panel have considered only the use of either acoustic control sources or vibration (point force) control sources. As such, a general understanding of the physical mechanism employed in this noise control technique has been lost. This paper presents a general analysis of the active control of sound radiation from a simply supported, baffled panel. It considers the use of both acoustic and vibration control sources. The minimization of the total sound power output is used as a criterion for actuator placement, and minimization of the sound pressure at a point is used for error microphone placement. Analytical results for minimization of the sound pressure at a point are verified experimentally. It is shown analytically that acoustic control sources operate by altering the radiation impedance seen by the vibrating panel. Vibration control sources operate by changing the panel velocity distribution, either decreasing the amplitude of the offending panel mode, and/or altering the temporal phasing of the panel modes, thereby decreasing the effective power radiation from the panel.

\title{
Session M. Underwater Acoustics II: High-Frequency Acoustic Imaging
}

\author{
Nicholas P. Chotiros, Chairman \\ Applied Research Laboratories, The University of Texas at Austin, P. O. Box 8029, Austin, Texas 78713-8029
}

Chairman's Introduction-2:00

Invited Papers

2:05

M1. The STRESS sonar experiment. Darrell R. Jackson and J. George Dworski (Applied Physics Laboratory, College of Ocean and Fishery Sciences, University of Washington, Seattle, WA 98195)

A high-frequency scanning sonar experiment has been conducted as part of the STRESS Program (sediment transport events: slopes and shelves). An autonomous platform was used to scan a circular region $100 \mathrm{~m}$ in radius with $5^{\circ}$ angular resolution. The acoustic frequency was $40 \mathrm{kHz}$ and the transmitted signal was an FM pulse of $2-\mathrm{kHz}$ bandwidth. The objective was to quantify changes in bottom scattering due to storm-induced sediment transport events. The acoustic experiment was conducted in water of depth $90 \mathrm{~m}$ at a silty site off the California coast. Other experiments measured environmental parameters such as sediment properties, bottom currents, and suspended sediment concentration. Scans were made at a rate of ten per day over a period of 13 weeks during the winter of 1988-1989. The acoustic data have been processed to determine scattering strength and the correlation between scans as a function of time separation. These results are presented in the form of images showing the evolution of the scattering process during quiet and stormy periods. Very little change is seen in the scattering strength, but the correlation magnitude exhibits a monotonic decrease that accelerated during a storm. The phase of the complex correlation provides a very sensitive indicator of small changes. Two applications of these phase data will be presented. [Work supported by ONR.]

M2. High-frequency acoustic imaging of the seafloor. C. de Moustier (Marine Physical Laboratory, Scripps Institution of Oceanography, A-005, La Jolla, CA 92093)

For decades, sidescan sonars have been the primary tool to obtain acoustic images of the seafloor. Such images provide qualitative information on the seafloor surveyed based on amplitude variations of the backscattered acoustic signals received. In the 1980 s, bathymetric sidescan sonar systems, capable of simultaneously producing acoustic images and measuring depth at numerous points across the swath, added a quantitative description of the seafloor in the form of a depth contour map. Similar claims can be made with multibeam echo sounders well known for their high-resolution swath bathymetry capabilities. Taking advantage of this high bathymetric resolution, the beamformed acoustic backscatter data can also be displayed as a geometrically correct acoustic image of the seafloor and provide textural information not available in the contoured bathy- 
metry of the same area. Likewise, knowledge of the bathymetry, particularly bottom slopes, is needed to correct for the angular dependence of seafloor acoustic backscatter and construct a map of acoustic backscattering strength over the area. Such a map will give clues to regional variations in lithologies.

\section{Contributed Papers}

\section{2:45}

M3. Evaluation and verification of bottom acoustic reverberation statistics predicted by the point scattering model. D. Alexandrou, G. Haralabus (Department of Electrical Engineering, Duke University, Durham, NC 27706), and C. de Moustier (Marine Physical Laboratory, Scripps Institution of Oceanography, A-005, La Jolla, CA 92093)

The point scattering model offers a parametrization of the reverberation envelope probability density function ( $p d f$ ) in terms of the average number of scatterers contributing to the return and the presence of a coherent component in the received process. Computer simulations were used to verify model predictions and to evaluate their usefulness in the context of seafloor classification. Central to the verification strategy was the successful solution of the inverse problem based on synthetic reverberation data. The average number of scatterers was determined from estimates of the kurtosis of the instantaneous reverberation pdf. The magnitude of a coherent component embedded in the scattered return was recovered from envelope histograms with the assistance of the Kolmogorov goodness-of-fit test. Following the verification study, the model was perturbed by introducing clustered and ordered distributions of scatterers in addition to the standard Poisson. The initial results indicate that the reverberation envelope pdf differs significantly for the three scatterer distributions. The clustered distribution led to a rapid increase in kurtosis, while the ordered distribution displayed evidence of intermittent coherent scattering. The usefulness of this parametrization was further tested with real reverberation data representing several distinct seafloor regimes. [Research funded by ONR.]

\section{3:00}

M4. Estimation of sediment volume scattering strength and absorption coefficient. Robert A. Stewart and Nicholas P. Chotiros (Applied Research Laboratories, The University of Texas at Austin, P. O. Box 8029, Austin, TX 78713-8029)

A simple theoretical model for estimating sediment volume scattering strength and absorption coefficient from the reverberation tail of an acoustic pulse observed by a point receiver within the ocean sediment is described. This model assumes an infinite plane wave entering the sediment at normal incidence and scattering isotropically from a uniform field of random inhomogeneities. The model predicts the magnitude and decay rate of the volume scattering at a point receiver in terms of the volume scattering strength and the absorption coefficient of the medium. By fitting the predictions of the model to experimentally observed volume scattering data, the volume scattering strengths and absorption coefficients of real sediments were inferred. Expeimental data were obtained from insediment acoustic probes deployed at sites off Kings Bay, GA, and off Panama City, FL. The absorption coefficient results were compared with corresponding measurements from core samples. [Work supported by ONT with NORDA management.]

\section{3:15}

M5. Plane-wave analysis of acoustic signals in a sandy sediment. Robert A. Altenburg, Carl Faulkner, and Nick Chotiros (Applied Research Laboratories, The University of Texas at Austin, Austin, TX 78713)
Acoustic pusles entering a sandy sediment through the water-sediment interface were observed. An acoustic projector located in the water column was used to insonify an array of sensors embedded in the sediment. Carrier frequencies of from 5 to $80 \mathrm{kHz}$ were projected toward the sediment interface at grazing angles below, near, and above the critical value. Sound-pressure levels and time of arrival differences associated with the passage of the acoustic pulse were determined from the sensor data. A weighted least-squares algorithm was used to fit a plane to the arrival time differences yielding wave-front direction and speed estimates. Estimates of acoustic attenuation and wave-front coherence were also obtained. [Work supported by ONT under NORDA management.]

\section{3:30}

M6. Remote sensing of particle motion by cross correlation of acoustic backscatter. Nicholas P. Chotiros (Applied Research Laboratories, The University of Texas at Austin, Austin, TX 78713-8029)

In a previous design study, it was determined that clouds of passive riders in the upper ocean, such as bubbles and suspended matter, could produce detectable and unique acoustic backscatter signatures. It is postulated that the acoustic signatures may be processed to yield the movement of the passive riders, hence image the motion of the water itself Methods based on cross correlation processing were developed. Computer simulations and experiments were carried out to test the methods. [Work supported by ONR.]

M7. Angular dependence of seafloor acoustic backscatter at $12 \mathrm{kHz}$ C. de Moustier (Marine Physical Laboratory, Scripps Institution of Oceanography, A-005, La Jolla, CA 92093) and D. Alexandrou (Department of Electrical Engineering, Duke University, Durham, NC 27706)

The angular dependence of seafloor acoustic backscatter measured with a $12-\mathrm{kHz}$ multi-narrow-beam echo sounder over a variety of seafloors, ranging in depth from 500 to $5000 \mathrm{~m}$, has been determined for incidence angles from 0 to $25 \mathrm{deg}$. The acoustic data consist of quadrature samples of the beamformed echoes received on each of the sixteen 2.66deg beams of a Sea Beam echo sounder. These data are subjected to adaptive noise canceling for sidelobe interference rejection and to beam-tobeam "decorrelation" to ensure that the returns are separated in angle with minimal cross talk between adjacent beams. The measurements are then corrected for geometric effects, taking into account the ensonified area for each beam, which varies as a function of the ship's roll and bottom slopes. It was found that apparent bottom slopes of less than $\mathbf{2}$ deg in the athwartships direction are noticeable in the data and must be compensated for. For lack of system calibration, the data are presented as relative mean energy levels in 1-deg bins from 0 - to about 25 -deg incidence. These results are compared with the results of a REVGEN computer simulation which faithfully reproduced the acoustic geometry of the Sea Beam system and generated scattered returns devoid of a specular component. Results are discussed based on geometric and physical arguments, and the ability to differentiate seafloor types using such angular dependence functions as assessed. [ Research funded by ONR.] 


\title{
Session N. Architectural Acoustics II: Acoustics in Performing Spaces
}

\author{
John W. Kopec, Chairman \\ Riverbank Acoustical Laboratories, 1512 Batavia Avenue, Geneva, Illinois 60134
}

Chairman's Introduction-3:00

\section{Contributed Papers}

3:05

N1. Is diffusion in concert halls a property of the volume or of the surface? James B. Lee (Concert Acoustics, Box 18017, Portland, OR 97218)

For nearly a century, the principal concept of the theory of concert halls has been that of a nearly uniform field of energy, generated by many reflections, that varies but slowly with time. This supposed uniformity and isotropy gave rise to the term "good diffusion," a property of the volume; it is difficult to quantify. An alternate approach is to apply the photometric concept of diffuse reflection at the surfaces: This is readily quantified, permits calculation of intensity at the audience, and posits nothing about the properties of the volume. Microcomputer programs to implement the concept are at hand and have been applied to a study of Boston Symphony Hall, renowned for its highly diffuse ceiling and walls. Modeling techniques, also borrowed from photometry, are available too: $1 / 50$ th scale architectural models are eminently suitable; note that models employing mirrors-specular reflectors-are not accurate representations of good concert halls.

\section{3:25}

N2. The organ as a teaching resource in architeetural acoustics. Bertram Y. Kinzey, Jr. (Department of Architecture, University of Florida, Gainesville, FL 32611)

The study of architectural acoustics must include an acquisition by students with limited or no musical background of a range of knowledge beginning with the harmonic structure of sound and its effect on timbre and continuing through an awareness of the ways, for better or worse, the architectural enclosure creates a reverberant field that affects various attributes such as blend, clarity, and fullness of tone and completes the musical sound that is heard. Aural experiences are essential if the student is to understand fully musical sound and the role of an architectural enclosure in its proper audition. The organ is an ideal instrument for demonstrations that give this experience. A series of live and recorded demonstrations using the organ is described which have been used over several years to show the relationship between music and architectural acoustics.

\section{3:45}

N3. Application of vector intensity techniques for the acoustical characterization of a baroque-style Italian theater. Domenico Stanzial, E. Carletti, I. Vecchi, and A. Fuschini (Cemoter, National Research Council of Italy, Via Canal Bianco 28, 44044 Ferrara, Italy)

Results concerning acoustical measurements carried out in the Municipal Theater of Ferrara, built in 1790, are here presented. Recent restoration works replaced the original wooden stage. A model, which describes the enclosed space as a retroactive linear system, has been developed to characterize rooms acoustics. Modifications of the acoustic field, induced by the new stage, were measured by means of the following objective tests: evaluation of the theater transfer function having related input and output, respectively, defined by the free-field acoustic power of a reference source and the measured power emitted by the same source placed on the stage surface; mapping of isointensity levels over the coupling area between stage and hall (proscenium plane); evaluation of acoustic-intensity-based transfer functions over a grid in the proscenium plane. A further description of the theater acoustics was obtained by intensity vector measurements over three parallel grids in the proscenium. Results of this additional characterization are presented as graphic views of acoustic intensity flow lines.

\section{$4 ; 05$}

N4. Verification of Bergeron's analytical method applied to room acoustics. Hidemaro Shimoda (Institute of Technology, Shimizu Corporation, Etchujima 3-4-17 koto-ku, Tokyo 135, Japan), Norinobu Yoshida, and Ichiro Fukai (Faculty of Engineering, Hokkaido University, N13 w8 Kita-ku, Sapporo 060, Japan)

Applicability of the Bergeron's method to room acoustics has been studied, and was found useful for a transient analysis in the time domain [J. Acoust. Soc. Am. Suppl. 1 84, S64 (1988)]. In this paper, a verification study of the proposed analytical method is presented. Transient time responses and reverberation times measured in a rectangular scale model were compared to the analytical results. The time responses at six positions in the room were observed by pulse excitation with 1/3-octave-band tone burst, and reverberation times were evaluated from these responses using Schroeder's integrated impulse method. Each time response at various points agreed very well with that of the scale-model experiment and the average of calculated reveberation times also agreed favorably with the experiment.

\section{$4: 25$}

N5. Temporal change in interaural cross-correlation functions due to signal dynamics. H. Yanagawa (Ohmori R\&D Laboratory, Pioneer Electronic Corporation, 15-5, 4-chome, Ohmori-Nishi, Ohta-ku, Tokyo 143, Japan)

Maximal values of transient interaural cross-correlation functions (TRICC) for simple burst signals were used earlier to analyze temporal changes in spatial impressions such as sound image localization and subjective diffuseness. These temporal changes were found to correspond with subjective experiences [Yanagawa et al., J. Acoust. Soc. Am. 84, 1728-1733 (1988) ]. for the present study, a sound signal was chosen that is closer to transient sources such as music or voice. It consists of two bursts of white noise with a silent pause inbetween that is shorter than the reverberation time of the room. TRICC values at the rise of the second white noise signal were analyzed to see how they changed with the length of the pause and with room volume. It was found that a shorter pause causes the fall (reverberation) of the initial sound burst to overlap the second, so that TRICC values are smaller, but less so when the room is smaller. These results clarify how temporal changes in TRICC closely reflect differences in rooms with regard to ease of sound image localization. 
N6. Separation of total transfer function phase into propagation and reverberant components. Lan Liu and Richard $\mathrm{H}$. Lyon (Department of Mechanical Engineering, Massachusetts Institute of Technology, Cambridge, MA 02139)

Previously reported theory of phase decomposition used the complex cepstrum to extract the all-pass phase from the total phase of acoustical space transfer functions. The all-pass phase is associated with the reverberant properties of two- and three-dimensional spaces. The dereverberation of acoustical signals in such spaces can be accomplished by this separation. This paper describes the results of some recent experimental studies of sound propagation in one-, two-, and three-dimensional spaces. A comparison of the expected and observed components of reverberant and propagation phase, with the results from these experiments, is presented.

TUESDAY AFTERNOON, 28 NOVEMBER 1989

ST. LOUIS BALLROOM A, 3:00 TO 5:12 P.M.

\author{
Session O. Speech Communication III: Fundamental Frequency and Intonation \\ George D. Allen, Chairman \\ Department of Audiology and Speech Science, Purdue University, West Lafayette, Indiana 47907
}

Contributed Papers

3:00

O1. Individual differences in voice quality perception. Jody Kreiman, Bruce R. Gerratt, and Kristin Precoda (West Los Angeles VA Medical Center, Audiology and Speech Pathology (126), Wilshire and Sawtelle Boulevards, Los Angeles, CA 90073, and UCLA School of Medicine, CHS 62-132, UCLA, Los Angeles, CA 90024)

Sixteen listeners judged the similarity of all possible pairs of 18 pathological voices and, in a separate session, 18 normal voices. Individual differences, multidimensional scaling was used to derive a separate perceptual space for each listener/voice set combination. These scaling solutions accounted for an average of $83 \%$ of the variance in similarity ratings for pathological voices, and $77 \%$ for normal voices. Listeners varied substantially in the acoustic characteristics they attended to when judging vocal similarity: Although all perceptual spaces included an $\boldsymbol{F} \mathbf{0}$ dimension, no other parameter was used by more than half the listeners, for either voice set. Listeners who shared common perceptual dimensions often differed in the way they used the same acoustic information. For example, $F 0$ was used as a continuous dimension by some listeners, and to sort voices into groups (high-and low-pitched groups, pathological and normal groups, etc.) by others; combinations of these strategies also occurred. Implications of these results for models of voice quality perception will be discussed.

\section{3:12}

02. The perception of the low-high (LH) tonal sequence. Kazue Hata (Speech Technology Laboratory, 3888 State Street, Santa Barbara, CA 93105) and Yoko Hasegawa (Department of Linguistics, University of California, Berkeley, CA 94720)

It has been reported that the primary cue for the HL tonal perception in Japanese is not the actual $F 0$ peak location but rather a falling $F 0$ contour. The $F 0$ fall may be significantly delayed, resulting in the $F 0$ peak within the L-toned syllable. Furthermore, it was found earlier thal (1) the later the $F \mathbf{0}$ fall in the L-toned syllable, the steeper the fall rate required and (2) the fall must begin within the first two-thirds of the duration of the vowel in the L-toned syllable. The present experiment investigates whether a lack of synchronization between $F 0$ change and syllable bound- ary can be found in the perception of the $\mathrm{LH}$ as well. Synthesized nonsense words /mamama/ were prepared in such a way that both the onset of $F 0$ rise and the $F 0$ peak occur at various locations, while maintaining the overall $F 0$ contour (level-rise-peak-slight fall). The stimuli were presented to native speakers of Japanese to determine the boundary between the categorical perception of LHH and LLH. The results show that the LH sequence is more constrained than the $\mathrm{HL}$ in terms of temporal alignment of $F 0$ change and the syllable boundary.

\section{3:24}

03. The frequency scale of intonation. Dik $\mathbf{J}$. Hermes and Joost $\mathbf{C}$. van Gestel (Institute for Perception Research/IPO, P. O. Box 513, NL 5600 MB Eindhoven, The Netherlands)

Results will be presented showing that accent-lending pitch movements are perceived on a critical-band scale. A sentence was resynthesized in two versions differing in pitch and in formant frequencies. The lowerpitched version sounded like a male voice, the higher one like a female voice. One syllable was rendered prominent by means of a pitch movement. The pitch contours of the two versions ran parallel on any of three frequency scales: a logarithmic frequency scale (semitones), a criticalband scale, or a linear frequency scale $(\mathrm{Hz})$. In $2 \mathrm{AFC}$ experiments, subjects indicated in which version the accented syllable was more prominent. Only when the excursions of the pitch movements were equal on a critical-band scale, the choice was random. In case of equality in semitones, subjects perceived the accent in the higher version as more prominent. In case of equality in $\mathrm{Hz}$, the accent in the lower version was perceived as more prominent. These results allow a perceptually more realistic measuring of the prominence of accented syllables. [Work supported by Instituut voor Doven, St-Michielsgestel, The Netherlands. ]

3:36

O4. Fundamental frequency and perceived prominence of accented syllables. J. Terken and R. Collier (Institute for Perception Research, P. O. Box 513, 5600MB Eindhoven, The Netherlands) 
In natural speech, accented words may differ as to the degree of perceived prominence. At the acoustic level, two aspects of fundamental frequency $(F 0)$ variation may be responsible for the perceived prominence differences: the magnitude of $F 0$ changes and the relative frequencies of $F 0$ maxima. Two experiments, with the same group of subjects, addressed the question of which aspect of $F 0$ better predicts perceived prominence. Both experiments used reiterant speech with synthesized $F 0$ contours. The speech materials consisted of 'mamamamamamama' utterances with F0 maxima on the second and penultimate syllables ("P1" and "P2," respectively). In one experiment, subjects adjusted the frequency of $P 2$ so that it was judged to have the same pitch as $\mathrm{Pl}$, for different rates of baseline declination. In the second experiment, subjects adjusted P2 so that it was judged to have the same prominence as P1, again for different declination rates. The results to be presented are relevant for refining the theory of pitch accentuation. For instance, if perceived prominence is predictable from $F 0$ maxima, both experiments should give the same results.

\section{3:48}

O5. Continuative intonation in Mandarin. J. S. Mirza (Department of Physics, National University of Singapore, Singapore 0511, Republic of Singapore)

The linguistic phenomenon of continuation in speech that manifests itself by modification of pitch contour in a preconjunction period was investigated for Mandarin. Mandarin is a tonal language; it has four tones. Its continuative intonation was investigated by examining selected parts of intonation contours prior to conjunctions for nine sentences each spoken by five speakers. Slopes of intonation contours across the words immediately before the conjunction were measured. It is reported here that the effect of continuation on Mandarin tones is highly dependent upon the type of conjunctions used. The continuation mostly drives up the slope values of Mandarin tones 2, 3, and 4, while the slope value of tone 1 is pushed down. The depression in slope value of tone 1 , however, cannot be taken seriously because its isolation mode value slope is close to zero and the percent changes can be erroneous. The continuation affects on the average tone 2 most of all $(+26 \%)$, then tone $3(+21 \%)$, and then tone $4(4 \%)$. The conjunction "and" has the greatest effect on the tones on the average $(+25 \%)$ followed by conjunction "but" $(23 \%)$ and "and then" (18\%).

\section{4:00}

06. Analysis and synthess of six voice qualities. $T$. V. Ananthapadmanabha (AT\&T Bell Laboratories, Murray Hill, NJ 07974) and Jo Estill (Voice Consultant, 408 West 57 Street, New York, NY 10019)

This is a preliminary study reporting on the acoustic analysis and synthesis of six selected voice qualities; speech, falsetto, low-larynx, twang, belting, and opera. The purpose of this investigation is to test the ability of the source-filter model to synthesize this wide range of voice qualities. Five vowels, $/ \mathrm{i} /, \mathrm{e} /, / \mathrm{a} /, / \mathrm{o} / \mathrm{,}$ and $/ \mathrm{u} /$, in six voice qualities spoken by a trained female speaker were recorded in a treated sound booth, allowing SPL and $F 0$ to free vary with a normal decay at the end of each token. These recorded signals were digitized directly onto a computer. Inverse filtering of vowel sounds was performed using the linear prediction technique. Parameters of voice source were extracted from the inverse filtered signal. Also, the spectral envelopes of the vowels were obtained. The six voice qualities were synthesized and compared with the original recording. Informal listening tests indicated little difference between the original and the synthesized tokens, thereby confirming the analysis procedure. To study the relative contributions of source and filter components, vowels were synthesized using the vocal tract transfer function appropriate for each quality but using an arbitrary voice source. The voice source was varied in two ways; (i) keeping the amplitude natural but pulse shape fixed and (ii) keeping both the amplitude and shape fixed. The differences in voice qualities were still distinguishable. Perceptually, these data seem to indicate that the spectral envelope is sufficient to synthesize these voice qualities. Physiologically, it would appear that glottal pulse shape is not significant. However, the spectral envelope may still contain components of the source such as subglottal coupling, spectral slope, and/or the effect of the laryngeal resonator on the vocal cavity transfer function. A demonstration tape will be presented.

\section{4:12}

O7. (Semi-)automatic pitch-synchronous computation of glottal flow. Jacques Koreman and Bert Cranen (Phonetics Institute, Nijmegen University, P. O. Box 9103, Nijmegen, The Netherlands)

Large-scale investigations of the aerodynamics of voice production are most easily performed using noninvasive measurement methods. Glottal airflow can be estimated by inverse filtering the mouth flow, measured with a pneumotachograph mask. A semi-automatic pitch-synchronous inverse filtering method was developed that combines the advantages of interactive inverse filtering and inverse filtering with fixed-filter settings: Our method yields accurate and calibrated estimates of glottal flow during VC and CV transitions, which are amenable for parametrization and can be used for bulk processing. Using glottal closing and opening moments derived from the electroglottogram, covariance LPC analysis on the closed glottis interval is employed to compute the optimal inverse filter for each period. For male speakers, the method yields quite stable waveforms. These will be parametrized in order to develop rules for a physiologically interpretable, time-varying synthetic voice source. The control parameters used are $F 0$, duty cycle, top-top flow amplitude, glottal leak area, and vertical phasing. A number of these parameters can be derived from both the EGG and the glottal flow. Comparison of parameter values from the two signals will give an indication of their reliability. The results will be discussed.

\section{4:24}

O8. Fundamental frequency database with linguistic and phonetic information. Masanobu Abe, Yoshinori Sagisaka (ATR Interpreting Telephony Research Laboratories, Sanpeidani Inuidani Seika-cho, Kyoto, 619-02 Japan), and Hisao Kuwabara (NHK Science and Technical Research Laboratories, Japan)

An important problem for speech science is the relationship between syntactic information, prosodic information, and fundamental frequency contour. To facilitate the study of the interaction among these three factors, all three have been coordinated in a continuous speech database. Specifications of the database are as follows. (1) Speech samples consist of 503 phoneme-balanced Japanese sentences spoken by a male professional announcer [Kuwabara et al., ICASSP '89, 560-563 (1989) ]. (2) Phonetic transcriptions at several levels of detail are provided [Takeda $e t a l$., Euro. Conf. Speech Technol. 2, 13-16 (1987)]. (3) Fundamental frequency is automatically extracted every $2.5 \mathrm{~ms}$ and extraction errors are corrected by hand. (4) The corresponding sentence is decomposed into constituent words and morphemes with lexical information such as inflectional categories and is assigned a tree structure. This information is semiautomatically generated from input texts. (5) Each utterance is segmented into minor phrases and each accent position is marked by listening to each utterance. This fundamental frequency database has been used to quantify fundamental frequency control factors and to show the effectiveness of this information.

\section{4:36}

O9. Vocal fundamental frequency: Variation by language, language group, and sex.Carolyn Wardrip-Fruin (Department of Communicative Disorders, California State University, Long Beach, CA 90840)

Vocal fundamental frequency was measured for speakers of five languages under three conditions (reading English, reading native language, 
and spontaneous speaking-native language). The samples were recorded in a sound-treated booth and analyzed by a Visipitch (Kay Elemetrics) frequency analyzer interfaced to an IBMxt computer. Preliminary analysis suggests that mean fundamental frequency was surprisingly similar across languages for the various speaking conditions, and that the mean fundamental was higher for reading than for speaking (as has been found in studies of English), but that there were significant differences between languages and by sex in standard deviation of the fundamental under the various speaking conditions (reading English, etc.). The results suggest that fundamental frequency is determined primarily by physiological factors with some linguistic variations.

\section{4:48}

010. On the mechanical properties of laryngeal muscles. Fariborz Alipour and Abdolali Najafi (Voice Acoustics and Biomechanics Laboratory, Department of Speech Pathology and Audiology, The University of lowa, Iowa City, IA 52242)

Active properties of canine laryngeal muscles were investigated through a series of experiments conducted in vitro. Samples of the thyroarytenoid medial and lateral muscles, also, cricothyroid pars recta and pars oblique, were dissected from dog larynges excised a few minutes before death and kept in Krebs solution at a temperature of $37 \pm 1{ }^{\circ} \mathrm{C}$ and a $p \mathrm{H}$ of $7.4 \pm 0.05$. Field stimulation with parallel-plate platinum electrodes was applied to study twitch and tetanic responses of these muscles in isometric conditions. The active force of each sample was recorded electronically with a dual servo system (ergometer). Results are reported on the twitch contraction times and half relaxation times of laryngeal muscles. Tetanic responses of these muscles were normalized and compared and results reported on tetanic $90 \%$ contraction and $90 \%$ relaxation times. It was found that the thyroarylenoid lateral muscle was the fastest muscle in the group with the mean twitch contraction time of 12.3 ms and mean half relaxation time of $11.9 \mathrm{~ms}$, and thyroarytenoid medial muscle had mean twitch contraction time of $21.2 \mathrm{~ms}$ and mean half relaxation time of $18.6 \mathrm{~ms}$. [Work supported by NINCDS Grant No. NS $16320-$ 07.]

\section{5:00}

011. Changes in vocal fold length with nerve stimulation in canine larynges. Jiaqi Jiang and Ingo $R$. Titze (Department of Speech Pathology and Audiology, University of Iowa, Iowa City, IA 52242)

Vocal fold length change and adductory movement of the glottis were obtained from seven canine larynges when the recurrent and the superior laryngeal nerve were stimulated bilaterally. A surgical procedure was used to make marks on the vocal fold for length measurements. Frame by frame analysis was used to get static and dynamic data on vocal fold length. The mean maximum elongation of the membranous vocal folds was $44.7 \%$ with 120 -ms elongation time and 140 -ms relaxation time. The mean maximum shortening of membranous vocal folds was $17.8 \%$, with $110-\mathrm{ms}$ contraction time and $115-\mathrm{ms}$ relaxation time. When both agonist and antagonist muscles were in maximum contraction, the mean elongation was $24.3 \%$. The length-time curve approximates an exponential function. Closing of glottis occurred in less than $35 \mathrm{~ms}$. The temporal results agree with earlier pitch change results. [Work supported by NIH Grant NS16320.] 


\title{
Session P. Noise II: Measurement of Employee Noise Exposure
}

\author{
Richard M. Guernsey, Chairman \\ R. M. Guernsey \& Associates, P.O. Box 1517, Morristown, New Jersey 07960-1517
}

Chairman's Introduction-8:00

\section{Invited Papers}

P1. Using noise dosimeters in an industrial noise control program. John Erdreich (Ostergaard Associates, 115 Bloomfield Avenue, Caldwell, NJ 07006)

Traditional use of the noise dosimeter to assess employee exposure is to satisfy a regulatory mandate. Issues of effects of microphone placement and statistical reliability of the measurements have limited the usefulness of exposure measures so obtained and furthermore, have deflected attention from the real utility of the device to provide critical information for noise reduction. Experience with measurements on maintenance workers shows that time histories that are provided by certain dosimeters allow the engineer to identify noise generating processes that produce the major components of the exposure. As a practical matter, the high-level processes that contribute several decibels to the time-weighted-average exposure are of greater concern than statistical variability induced by other factors. For this reason, acquisition of time histories in employee exposure assessment is encouraged.

\section{$8: 30$}

P2. Monitoring noise exposure using employee movement analysis techniques. Thomas S. Bragg (Noise Unlimited, Inc., 9 Saddle Road, Cedar Knolls, NJ 07927)

In most industrial activity, employee work routines vary from one day to another or from week to week. The United States Occupational Safety and Health Administration (OSHA), recognizing this fact, has specified that an employee's long-term noise exposure should be expressed as a summation of partial exposures to varying sound levels. A technique has been developed that analyzes employee movement, breaking an average day into a series of locations or activities, each with an associated time duration. Sound level data are then acquired for each location or activity. A computer is then used to calculate the partial exposure, as defined by any criterion, for each location or activity and these are summed to express the overall exposure level for each employee job function. The computer also generates a list of noise control priorities and the minimum amount of noise reduction required at each location to achieve compliance with the selected criterion. Maximum priority is given to the location which, when attenuated, will give most benefit to most employees. Determining these minimum reduction levels is most important, as the cost and feasibility of noise control are directly related to the amount of reduction required.

P3. A system to monitor the noise exposure of persons wearing headsets. W. K. Van Moorhem and K. S. Woo (Mechanical Engineering Department, University of Utah, Salt Lake City, UT 84112)

Although legislation limiting noise exposure in the work place applies to individuals who wear headsets as part of their employment, no method for measuring the noise exposure of these individuals has previously existed. A system for carrying out this measurement has been developed. This system is indirect, in that the electrical signal to the headset is measured, the transfer function of the headset, ear, and dosimeter microphone is applied to the measured signal, and the resulting electrical signal input to a dosimeter. This approach requires the transfer function of the headset be measured for each brand and model that is used, but eliminates the need for in-ear microphones of the more direct approach. The transfer function of the headset is measured using an acoustic manikin head. A limited number of headsets brands and models have been used with this system to investigate both the variations between models and within a model. [Work supported by Department of Labor.] 
P4. Probe microphone techniques for determining exposure to noise. Mead C. Killion (Etymotic Research, 61 Martin Lane, Elk Grove Village, IL 60007)

Traditional dosimeter measures, using a microphone in the sound field, provide little information about the actual noise exposure experienced by a subject wearing earplugs, earmuffs, earphones (traditional or insert), or someone exposed to a highly directional or local noise source. In these instances, the noise at the eardrum would appear to be a more useful measure, but cannot be directly compared to traditional damage risk criteria because the latter are based on measurements in a diffuse sound field. If a simple bridged-T filter network [Killion, J. Audio Eng. Soc. 27, 13-16 (1979)] correcting for the transfer function between the diffuse field and the eardrum is interposed between a flat-frequency-response probe microphone and a dosimeter, the resulting dosimeter indication can be compared directly to traditional damage risk criteria. Techniques for minimizing the potential errors in such measurements will be discussed.

P5. Measurement of hearing protector attenuation using the physical ear attenuation test (PEAT). Kevin Michael (Department of Communication Disorders, The Pennsylvania State University, 3F Moore Building, University Park, PA 16802)

The attenuation provided by a sample of circumaural hearing protectors (ear muffs) was measured using the physical ear attenuation test (PEAT). The PEAT utilized a small electret microphone mounted in a silicon ear plug in the outer ear of a human subject. The subjects were exposed to a high level (about $100 \mathrm{dBA}$ ) of pink noise with and without the hearing protector fitted. The attenuation provided by the protector was calculated by subtracting the spectrum measured under the protector from the spectrum measured without the protector. The results of the PEAT evaluation were compared to the results obtained using the test method described in ANSI Standard S12.6-1984, the Method for the Measurement of the Real-Ear Attenuation of Hearing Protectors. Agreement was found between the methods for the test frequencies from 250 to $8000 \mathrm{~Hz}$. The PEAT results at $125 \mathrm{~Hz}$ were lower than the results obtained with the ANSI S12.6-1984 method.

10:10-10:30

Break

\section{Contributed Papers}

\section{0:30}

P6. Comparison of ANSI noise dosimeters and IEC personal sound exposure meters. Robert Krug (Cirrus Research, Inc., 6818 W. State Street, Suite 170, Wauwatosa, WI 53213)

ANSI and IEC have prepared draft standards on meters to measure workers sound exposure. The standards have a number of similarities. Both are based on IEC 804, integrating-averaging sound level meters, and both are intended to specify meters worn on a worker and to measure noise throughout a work day. The ANSI draft specifies meters with exchange rates of 3,4 , or $5 \mathrm{~dB}$, with a fast or slow time constant and a threshold. The IEC draft specifies only a $3-\mathrm{dB}$ exchange rate with no time constant or threshold. The ANSI draft specifies a read out in percent dose while the IEC draft is developed around Pascal squared hours. For continuous noise above the threshold, the reading can be converted from one set of units to the other. For intermittent and impulsive, a noise significant difference will occur.

\section{0:45}

P7. Low-frequency sound intensity measurement and free-field soundpressure propagation. William D. Gallagher and Lawrence F. Heitkamp (Engineering Specialties Corporation, 8147 Delmar, Suite 207, St. Louis, MO 63130)

Classical acoustical equations relate the sound power of a source to sound-pressure level at a given distance from that source. Sound intensity of a source can be measured, sound power determined by integration over the radiation area, and the sound-pressure levels at a distance from the source can be calculated. An assumption is made when the sound power to sound-pressure calculation is made, that at low frequencies $(20-100 \mathrm{~Hz})$ the waves have propagated and the resultant sound-pressure level is from the source. This work tests the reliability of that assumption. A speaker was suspended inside three different size boxes, and powered by a noise generator bandpassed at discrete frequencies. Sound intensity measurements were recorded near field at the same discrete frequency. Free-field sound pressure measurements were then recorded at distances from the boxes. This paper reports the results of the measurements.

\section{1:00}

P8. Reverberation and noise propagation measurements in a power plant. Frank H. Brittain and Ralph W. Passage (Bechtel Corporation, P.O. Box 3965, San Francisco, CA 94119)

The acoustical characteristics of empty spaces are relatively well known. An understanding of room acoustics is important both for designing spaces and for controlling noise from equipment in enclosed spaces. Power plants and similar industrial plants pose a challenge because their spaces are not only very large, but also "full" of equipment. These spaces contain a high concentration of rotating equipment, piping, structural steel, cable trays, gratings, and tanks. Measurements of reverberation time and noise propagation were made in a power plant. The equipment in these spaces changes the acoustical characteristics of the spaces. These 
measurements show that sound absorption properties of the spaces are greater than are expected from published sound absorption data and theories of room acoustics. Implications of the results for noise control of power and other industrial plants are discussed.

\section{1:15}

P9. The aircraft noise-induced permanent threshold shift. ' $W u$ Yongxiang, Wang Bing-guang, and Wang Xin-yu (Institute of Aviation Medicine, Air Force, PLA, Xi Diao Yu Tai 30 (East), Beijing, People's Republic of China)

The hearing of 410 groundcrew who had worked under 100-130 $\mathrm{dB}(\mathrm{A})$ aircraft noise was measured. The groundcrew were divided into four groups by the length of service: less than 5 years, 5-9 years, 10-14 years, and 15-20 years. Their audiograms were taken with a Kamplex audiometer in a soundproof mobile cabin, in which background noise was 23-25 $\mathrm{dB}(\mathrm{A})$. One-hundred and forty seven military men of comparable age who had not been exposed to excessive noise were selected as a control group. Their audiograms were taken in the same way. The results indicated that the hearing injury was an actual menace to the groundcrew. Among them the high-frequency hearing loss and the noise deafness made up $46.1 \%$ and $6.1 \%$, respectively. Compared with the control group of the same age, the differences of the hearing average value of each frequency were significant. Their hearing injury was aggravated with the length of service. The high-frequency hearing losses of the four groups, less than 5 years, 5-9 years, 10-14 years, and 15-20 years, were $32.7 \%, 47.3 \%$, $54.4 \%$, and $67.3 \%$, respectively. In order to prevent hearing damage, the groundcrew ought to wear ear protectors and the ground noise suppressors should be used when the aircraft engine is operating. It was discovered that the " $V$ "-shaped depression was not at $4 \mathrm{kHz}$ but at $6 \mathrm{kHz}$ in 410 groundcrew. There were similar phenomena in domestic and foreign literatures. The former views concerning the pathogenesis of the "4-kHz depression," therefore, is open to question. This is a quite interesting subject that warrants further study.

11:30

P10. Hearing conservation programs (HCPs): The effectiveness of one company's HCP in a 12-h workshift environment. Jacqueline L. Reynolds (Department of Industrial Engineering, NCSU, Raleigh, NC 27695-7906), Larry H. Royster (Department of Mechanical
Engineering, NCSU, Raleigh, NC 27695-7910), and Richard G. Pearson (Department of Industrial Engineering, NCSU, Raleigh, NC 276957906)

A hearing conservation program (HCP), originally designed for an 8h workshift schedule, was evaluated at a plant site where a 12-h workshift schedule is now utilized. The study included three phases: a noise analysis of the work environment, HCP evaluation using audiometric database analysis (ADBA), and an evaluation of the hearing protection devices (HPDs) used at the facility by measuring temporary threshold shift (TTS) and applying ADBA procedures. The overall measured employee TWA in the process area where the TTS study was conducted was 92 dBA. The ADBA results indicated that the existing HCP is, at best, marginal. The most likely causes of this less than desirable rating are inadequate audiometric testing procedures and inadequate HPD utilization. It was concluded that the introduction of the $12-\mathrm{h}$ workshift has had no impact on the present effectiveness of the HCP. All three HPDs in use at the facility (3-M foam earplug, E-A-R foam earplug, and Bilsom Soft earplug) offered effective protection from noise at all audiometric test frequencies $(0.5$ to $6 \mathrm{kHz}$ ) except $0.5 \mathrm{kHz}$. All three HPD wearer groups exhibited mean TTS at $0.5 \mathrm{kHz}$, which was significantly different from zero at the 0.05 level for the E-A-R and 3-M wearer groups.

\section{$11: 45$}

P11. An improved modeling technique for high-order acoustic spectra. Vijay Raman, Osama Mohammed, Kang Yen, and Kurt Ramdin (Department of Electrical Engineering, Florida International University, Miami, FL 33199)

The analysis and modeling of noisy environments are considered in this work. Parametric modeling is often the most convenient, since it can be used as a basis for parametric filtering and adaptive filtering for noise reduction. The earlier work of these researchers dealt with the development of autoregressive (AR) as well as the autoregressive-moving-average (ARMA) modeling of a data set taken from digitized recordings of a typical industrial environment. It was shown therein that the order of the model required is extremely high due to the periodic components, and is therefore not as useful as expected. In this work, a new approach is developed to model periodic components and the random part sequentially, not separately. This method is able to model the data set with much fewer coefficients than in the standard parametric models, and is useful in designing the noise-reduction filter. [ Work supported by the Florida High Technology and Industry Council.]

WEDNESDAY MORNING, 29 NOVEMBER 1989

ST. LOUIS BALLROOM G, 8:00 A.M. TO 12:00 NOON

\title{
Session Q. Physical Acoustics III and Bioresponse to Vibration II: Biophysical Mechanisms as Bases of Safety Criteria for Medical Ultrasound II
}

\author{
Edwin L. Carstensen, Chairman \\ Rochester Center for Biomedical Ultrasound, University of Rochester, Rochester, New York 14627
}

Contributed Papers

8:00

Q1. Observation of acoustic cavitation in excised canine urinary bladders. J. B. Fowlkes, P. L. Carson, E. H. Chiang, and J. M. Rubin (Department of Radiology, Division of Radiological Physics and
Engineering, University of Michigan Medical Center, Ann Arbor, MI 48109-0553)

A high-intensity ultrasonic field has been used to generate bubbles within urinary bladders excised from dogs. Following the exposure, bub- 
bles were easily visualized using an ATL UltraMark 9 diagnostic scanner with a 5-MHz mechanical sector scanhead. Scattering of the high-intensity ultrasound by the bubbles was also observed as uniformly high amplitude scan lines during the irradiation. The bladders were removed surgically after tying of the ureters and urethra to prevent urine loss and exposure to external contaminants. Each bladder was sealed in a plastic bag filled with a degassed aqueous solution of sodium chloride $(0.9 \%$ by weight) and sodium azide ( $1 \mathrm{~g} /$ liter $)$. A bag containing a bladder was centered in a sealed degassed water path at the common focal point of a 7 $\mathrm{cm}$-diam, $500-\mathrm{kHz}$ transducer, and a $10-\mathrm{cm}$-diam brass reflector. The transducer and reflector were both focused at $10 \mathrm{~cm}$ and were aligned coaxially. Exposures were $10 \mathrm{~s}$ in duration but intensity or pressure values were not available as of the writing of this abstract. No significant discoloration of the exterior bladder wall was observed within the beam path. Results of this study suggest that bubbles might be externally generated in vivo for use as echo contrast agents and for studying the bioeffects of acoustic cavitation.

\section{8:15}

Q2. Excitation of surface mode activity of bubbles in ultrasonic fields. Robert K. Gould (Department of Physics, Middlebury College, Middlebury, VT 05753)

When a gaseous bubble finds itself in a liquid in which an ultrasonic field exists, the bubble may undergo radial, orderly surface mode or chaotic surface oscillations depending on the size of the bubble, strength of the sound field, and other parameters such as viscosity of the liquid and frequency of the sound. In the event of purely radial oscillations there is little acoustic streaming in the vicinity of the bubble. However, when bubble surface activity is present, vigorous streaming will occur near the bubble. Experimental studies of a bubble levitated in a $20-\mathrm{kHz}$ standing-wave sound field in a chamber of water have included the obtaining of stroboscopic movies of bubbles undergoing orderly surface modal vibrations of orders 2,3 , and 4 , there being no existence of mode 1 . The frequency of the surface oscillations is half that of the sound field. Movies of modal oscillations 2, 3, and 4 will be shown. Movies of mode 4 were obtained for the first time this year by George E. Lawrence, while carrying out his senior thesis research.

\section{8:30}

Q3. Radial oscillations of gas bubbles in viscoelastic materials. Charles C. Church and Ronald A. Roy (National Center for Physical Acoustics, University, MS 38677)

Most previous studies of acoustic cavitation in biological tissue have treated the material surrounding the bubble as a Newtonian fluid with sometimes increased viscosity. The present work describes the derivation of a generalized Rayleigh-Plesset equation for a gas bubble in a continuous viscoelastic material. Viscoelasticity is modeled to include either stress relaxation (Maxwell fluid) or aftereffect (Voigt fluid). Several cases are considered: (1) a bubble with a (a) viscous shell suspended in a Newtonian fluid, (b) viscoelastic shell suspended in a Newtonian fluid, (c) viscoelastic shell suspended in a viscoelastic fluid; and (2) a "clean" bubble suspended in a (a) viscous or (b) viscoelastic fluid. In general, the effect of viscoelasticity is to decrease the resonance frequency and increase the threshold for transient cavitation. Preliminary measurements of cavitation thresholds in a liquid seeded with Albunex ${ }^{(9)}$ stabilized microbubbles are presented and interpreted in light of the theory. [Work supported by NIH.]

\section{8:45}

Q4. The threshold of thermally significant cavitation in muscle in vivo. Kullervo Hynynen (Department of Radiation Oncology, University of Arizona, Health Sciences Center, Tucson, AZ 85724)

The transient cavitation threshold was measured by dogs' thigh muscles in vivo during a 1-s exposure to continuous or pulsed ultrasound at frequencies between 0.25 and $1.68 \mathrm{MHz}$. The temperature elevation at the focal zone at the depth of $5 \mathrm{~cm}$ in the tissues was measured during the sound pulse using a twisted pair thermocouple (wire diameter $50 \mu \mathrm{m}$ ). A focused hydrophone was also used to sense the subharmonic emission from the focal zone. At each thermocouple location the pulses were repeated with increasing applied acoustic power until the temperature elevation became irregular with sudden temperature peaks during the 1-s pulse and with an appearance of subharmonic emission of noise. Similar events were also observed at higher intensities during low-duty cycle pulses, although not with pulses including only 1 or 2 cycles. These results indicate that transient cavitation can appear at intensities that could be reached during clinical scanned focused ultrasound hyperthermia $(400-$ $500 \mathrm{Wcn}^{-2}$ at $1 \mathrm{MHz}$ ) and thus can significantly modify the temperature distribution and the biological effects of the treatment. [Work supported by NCI Grant No. CA 46627.]

\section{9:00}

Q5. Comparison of levels for ultrasonic cavitation in neonatal mice and other mammals. Leon A. Frizzell (Department of Electrical and Computer Engineering, University of Illinois, 1406 West Green Street, Urbana, IL 61801)

The level for cavitation involvement in hind limb paralysis of the mouse neonate has been determined at $10^{\circ} \mathrm{C}$ and $37^{\circ} \mathrm{C}$ at $1 \mathrm{MHz}$. These levels are compared to levels for cavitation as reported in other mammalian systems. Results have also been obtained for pulsed exposures, and a comparison is made between results from continuous wave and pulsed exposures. It is suggested that the pulse average intensity and total sound on time may be more useful parameters (as opposed to time average intensity and exposure duration) for comparing pulsed and continuous wave cavitation results within certain exposure regimes. This may prove to be a useful approach to the incorporation of continuous wave results, relating to cavitation, in the determination of safe levels of exposure to diagnostic ultrasound.

\section{9:15}

Q6. Biophysical mechanisms in the formulation of Canadian guidelines for diagnostic ultrasound devices. Stephen H. P. Bly and D. A. Morison (Department of National Health and Welfare, Health Protection Building, Room 66, Tunney's Pasture, Ottawa, Ontario K1A OL2, Canada)

A mechanistic assessment of ultrasound safety is presented as a method to formulate guidelines for diagnostic ultrasound devices. This approach was adopted since surveys had indicated that ultrasound output levels from some existing devices were higher than the no-effect levels found in epidemiological studies and studies of mammalian bioeffects. Existing studies on two mechanisms, heating and cavitation, were examined. It was found that knowledge of the cavitation mechanisms in vitro and in nonmammalian model systems could not be extrapolated to predict cavitation thresholds in humans. For the thermal mechanism, sufficient data were available [AIUM Bioeffects Committee, J. Ultrasound Med. 7, S1-S38 (1988)] to formulate a guideline. The guideline recommends that, for diagnostic ultrasound devices used for fetal examinations and yielding free-field spatial peak temporal average intensities greater than $500 \mathrm{~mW} / \mathrm{cm}^{2}$, estimates should be provided of maximum local temperature elevations in fetal tissues.

\section{9:30}

Q7. Intensity gain criteria for focused ultrasound tumor hyperthermia: Theory and in vitro experiments. Brian J. Davis and Padmakar P. Lele (Department of Mechanical Engineering, Massachusetts Institute of Technology, Cambridge, MA 02139)

Plano-concave lenses and concave radiators used in scanning focused ultrasound systems have well-established capabilities for deep and selective tumor hyperthermia. The focal intensity gain, a good predictor of 
selective heating, is expressed as a function of the treatment depth below a planar beam portal integument, frequency-dependent tissue absorption rate, wavenumber, and aperture angle. From this expression novel and useful relations are derived for the maximum focal gain as a function of system specific maximum aperture angle, optimum frequency, and other parameters. Temperature distributions from in vitro and tissue phantom experiments designed to establish minimum gain criteria for selective heating with "pivoted" and circular scanned focused beams are presented. Measured intensity fields are compared with numerical models that aim to (1) solve the diffraction pattern of an absorbing spherical lens coupled to a clamped piston radiator, (2) predict the intensity field of a focused beam in an absorbing medium, and ( 3 ) account for lens propagation that is not parallel to the beam axis. [Work supported by USPHS Grant No. 31303 and the Lutheran Brotherhood.]

\section{9:45-10:00 \\ Break}

\section{0:00}

Q8. Estimation of mean scatterer size from sparse, random distributions of polystyrene spheres in solution via Mie scattering and cepstral signal processing: Theory and experimental results. C. R. Meyer, J. B. Fowlkes, P. L. Carson, H. Wang, and H. Chan (Department of Radiology, Division of Radiological Physics and Engineering, University of Michigan Hospitals, Ann Arbor, MI 48109-0553)

Four solutions of narrowly distributed polystyrene spheres of one diameter, either $41,136,271$, or $381 \mu \mathrm{m}$, sparsely suspended in degassed water, were imaged using multiple 5- and 7.5- $\mathrm{MHz}$ medical ultrasonic transducers. Radio-frequency backscattering data from the 136-, 271-, and $381-\mu \mathrm{m}$ solutions were processed using the data from the $41-\mu \mathrm{m}$ solution as a volumetric reference to correct for transducer diffraction effects and acquisition system gain vibrations. The power cepstrum of the mean log difference spectra averaged over all overlapping data segments was computed and corrected for the effects of attenuation and Rayleigh scattering in the reference. Abcissas of the cepstral peaks were plotted versus scatterer diameter. The zero-intercept, linear regression line for the longer-lag peaks yielded a slope of $3.4 \mu \mathrm{s} / \mathrm{mm}, r^{2}=0.997$, corresponding to a Mie-region periodicity in backscattered cross section of $k a=0.6$. This value is in excellent agreement with calculations based on the scattering theory of Faran, which includes shear waves and yields a periodicity of $k a=0.66$.

\section{0:15}

Q9. Thresholds for surface wave generation on acoustically levitated gas bubbles. Steve Horsburgh, R. Glynn Holt, and Lawrence A. Crum (National Center for Physical Acoustics, University of Mississippi, University, MS 38677)

When a gas bubble is acoustically levitated in a standing wave, the spherically symmetric volume oscillations can readily couple into surface waves [M. Strasberg and T. B. Benjamin, J. Acoust. Soc. Am. 30, 697
(1958) ]. Using a light scattering technique, the threshold for inception of individual surface waves was examined and the results were compared with both analytical and numerical calculations of this threshold. The observed and calculated values are generally in agreement although the identity of the surface wave mode is difficult to determine. [Work supported in part by the Office of Naval Research.]

\section{0:30}

Q10. The application of Hamilton's principle to a bubbly liquid. J. A. Hawkins, Jr. (Applied Research Laboratories, The University of Texas at Austin, Austin, TX 78713) and A. Bedford (Department of Aerospace Engineering and Engineering Mechanics, The University of Texas at Austin, Austin, TX 78712)

Naturally occurring bubble populations have a distribution of bubble sizes. Well-established continuum theories exist for the propagation of acoustic waves through a bubbly liquid with a single bubble size. The application of these theories to bubbly liquids with several sizes is necessarily limited. Here two approaches are described based on Hamilton's principle for analyzing the acoustics of bubbly liquids containing a distribution of bubble sizes. First, the mixture is treated as a liquid containing a bubble "continua" of several discrete sizes. The analysis leads to $N+2$ ( $N$ is the number of different bubble sizes) simultaneous equations in the motion, density, and volume fraction, which must be solved numerically. The system was solved for one, two, three, and five different bubble sizes. The second approach is to assume that a continuous distribution of bubble sizes exists in the liquid. Surprisingly, the second approach leads to a relatively simple expression for the wavenumber. It was shown that the phase velocity and the attenuation of acoustic waves can be determined for an arbitrary distribution of bubble sizes. The two approaches are shown to agree in the limiting case of discrete bubble sizes and to agree with the result obtained by Commander and Prosperetti [K. W. Commander and A. Prosperetti, J. Acoust. Soc. Am. 85, 732-746 (1989)]. [Work supported by ONR, IR\&D Program of ARL:UT.] 
Application of Information on Mechanisms in the Development of Exposure Criteria

PANEL CHAIRMAN: A. R. Williams

University of Manchester

Department of Medical Biophysics

Manchester M13 9PT, United Kingdom

PANEL MEMBERS: Stephen H. P. Bly

Bureau of Radiation and Medical Devices

Room 233, Environmental Health Centre

Ottawa, Ontario K1A OL2, Canada

Mel E. Stratmeyer

Center for Devices and Radiological Health

12709 Twinbrook Parkway (HFZ-112)

Rockville, Maryland 20857

Gail ter Haar

Institute of Cancer Research

Clifton Avenue

Sutton, Surrey SM2 5PX, United Kingdom

Marvin C. Ziskin

Department of Diagnostic Imaging

Temple University School of Medicine

Philadelphia, Pennsylvania 19140

WEDNESDAY MORNING, 29 NOVEMBER 1989

ST. LOUIS BALLROOM B, 8:00 A.M. TO 12:00 NOON

\section{Session R. Physiological Acoustics I and Psychological Acoustics III: Ear Canal to Cortex: Quantifying Auditory Mechanisms}

Julius L. Goldstein, Chairman

Central Institute for the Deaf, 818 South Euclid Avenue, St. Louis, Missouri 63110

Contributed Papers

8:00

R1. Measurement of the local power reflection coefficient in the ear canal using the two-microphone method. W. J. Murphy, A. Tubis (Department of Physics, Purdue University, West Lafayette, IN 47907), and G. R. Long (Department of Audiology and Speech Sciences, Purdue University, West Lafayette, IN 47907)

The local power reflection coefficient in the ear canals of humans has been measured using the two-microphone technique [W. J. Murphy, A. Tubis, and G. R. Long, J. Acoust. Soc. Am. Suppl. 1 82, S70 (1987) ]. The accuracy of the calibration technique and the assessment of the random and bias errors associated with the reflectance have been investigated. The use of bandlimited noise as a stimulus results in decreased coherence between the two microphone signals relative to the coherence obtained with sinusoidal or other deterministic signals (e.g., a chirp signal) at high frequencies. The effects on the coherence and the reflectance due to sinusoidal chirp and bandlimited stimuli are presented. The measurements were performed in an acrylic hard-terminated tube. As a test of the assumption of local one dimensionality of the ear canal, the reflectance was measured as a function of the insertion depth of the two-microphone probe in several subjects. At high frequencies, the measured reflectance was found to vary significantly in ear canals with contorted geometries. In relatively straight ear canals, the measured reflectance was found to vary by less than $10 \%$ for changes of $5 \mathrm{~mm}$ in the insertion depth. [Work supported by a grant from the Deafness Research Foundation.] 
less $(8.1 \mathrm{~dB})$ energy was required for elicitation of threshold response in the shallow position as compared to the two other. However, no statistically significant differences were found between the most appropriate (full and intermediate) insertion depths at any tested frequency. Although REAT values showed increased attenuation for the frequencies $0.125-1 \mathrm{kHz}$ as the eartip was inserted more deeply in the ear canal, at all three insertion depths over all tested frequencies, values were more than $15 \mathrm{~dB}$ greater than the attenuation obtained with the conventional earphone. York, 1985), pp. 46-51] suggests that a very large fraction of the incident acoustic power in the cat ear canal is absorbed at the eardrum. In some animals, the high efficiency extends over a very wide frequency range from $300 \mathrm{~Hz}-30 \mathrm{kHz}$. These findings are sensitive to the condition of the animal and are subject to relatively large interanimal variations, particularly at high frequencies. Theoretical models describing the function of the middle ear differ significantly in predicting the power absorption and in predicting the fraction of the energy flux dissipated within the eardrum itself. Some insight into the behavior of the eardrum can be gained by studying the power absorption properties when the ossicular chain is immobilized or removed. In doing so, the dynamics of the ossicular chain, and to a large extent the dynamics of the cochlea, is removed from the system, leaving the eardrum coupled to the acoustics in the ear canal and in the tympanic cavity. The power absorption potential of the eardrum for several internal dissipation mechanisms is investigated using three-dimensional representations of the eardrum, ear-canal acoustics, and middle-ear acoustics. Results imply that the acoustic behavior of the middle-ear air chamber(s) has a profound influence on energy transmission and absorption potential of the eardrum.

R3. Impedance measurements in the ear canal. Sunil Puria) (Department of Electrical Engineering, The City College of CUNY, New York, NY 10036) and Jont B. Allen (AT\&T Bell Laboratories, 600 Mountain Avenue, Murray Hill, NJ 07974)

The objective of the present work is to make accurate impedance estimates at the eardrum $Z_{\mathrm{Im}}(\omega)$. This is done by measuring the thevenin parameters of an earphone using a four-cavity calibration method [J. B. Allen, "Measurement of eardrum acoustic impedance," in Peripheral Auditory Mechanisms, edited by J. B. Allen et al. (1985) ] for frequencies up to $15 \mathrm{kHz}$. The calibrated earphone is then used to measure the ear canal impedance $Z_{c}(\omega)$ near the ear canal entrance. To transform $Z_{c}$ to $Z_{l m}$, the ear canal cavity has been modeled. The following effects were accounted for by the model: (a) the jump in area from the earphone calibration cavity to that of the ear canal, (b) the distance to the eardrum, (c) variations in cross-sectional ear canal area, and (d) effects due to ear canal wall impedances. It is desirable that parameters of the ear canal be derivable from $Z_{e}$. Preliminary results isolating each of these effects first in tubes and then in real ear canals are presented. a) Presently, consultant to AT\&T Bell Laboratories Acoustics Research Department.

\section{8:45}

R4. Influence of insertion depth of an insert earphone on hearing threshold levels and on real ear attenuation values. Fredrik Lindgren and Elliott H. Berger (E-A-R Division, Cabot Corporation, P. O. Box 68898, Indianapolis, IN 46268-0898)

The influence on pure-tone hearing threshold levels (HTLs) of the insertion depth of the coupling foam eartip of EARTONE ${ }^{\text {TM }} 3 \mathrm{~A}$ insert audiometric earphones was investigated in 16 subjects using three distinctly different insertion depths: shallow, intermediate, and full. HTLs were also measured using conventional earphones, model TDH-50P with MX/41-AR cushions. In addition, sound field real ear at threshold (REAT) values re: ANSI S12.6-1984 were recorded in each condition. The results showed that there was a tendency toward poorer HTLs as the eartip was positioned further away from the eardrum, with the difference between the three widely disparate insertion depths amounting to 1.3-5.3 $\mathrm{dB}$ in the frequency range $0.5-6 \mathrm{kHz}$. In contrast, at $8 \mathrm{kHz}$, significantly

9:00

R5. Distortion product emissions as predictors of frequency-specific auditory thresholds. Barry P. Kimberley and David A. Nelson (Department of Otolaryngology, 2630 University A venue SE, Room 134, University of Minnesota, Minneapolis, MN 55414)

Distortion product emission (DPE) input-output functions were measured at seven frequency regions, covering a range from $700-6000 \mathrm{~Hz}$, for each of 50 ears. Auditory thresholds at each frequency region were determined using adaptive, forced-choice psychophysical techniques. One-half of the subjects had auditory thresholds less than $25 \mathrm{~dB} \mathrm{HL}$ at every frequency. One-half of the subjects had a variety of patterns of pure sensorineural loss. The stimulus level required to just raise an emission above the noise floor is a feature that can be measured directly from a DPE input-output function. This feature is called "emission threshold." When frequency-specific auditory thresholds were compared with corresponding emission thresholds, a close correlation was found over a range of auditory thresholds from -6 to $+55 \mathrm{~dB}$ SPL. A linear regression between auditory and emission threshold has a slope of 1.0 and a regression coefficient of 0.86 . However because emission threshold does not grow as quickly as auditory threshold at high threshold values, a better correlation of 0.9 is obtained by allowing a cubic nonlinear fit. Emission thresholds correlated with auditory thresholds in a similar way at each frequency region. Thus DPE measurements appear to be well correlated with frequency-specific auditory thresholds. This finding suggests an enhanced clinical value of DPE measurement. DPE measures appear able to predict frequency-specific sensory thresholds with reasonable accuracy over a moderate range sensory thresholds. [Work supported by American Otologic Society.]

\section{9:15}

R6. Spontaneous otoacoustic emissions: Evidence for a cricadian rhythm of frequency variation. Holly S. Haggerty (Division of Otolaryngology, R-135, Stanford University School of Medicine, Stanford, CA 94305)

An investigation of the hour-by-hour frequency stability of spontaneous otocacoustic emissions was conducted over a $24-\mathrm{h}$ period. Two emissions were recorded from each of two audiometrically normal adults. Time series analysis was used to model the autocorrelation structure of the measurements, to resolve each 24 -h series of measurements into cyclical components of various periodicities, and to test the statistical significance of given cycles within the spectrum of each series. Results showed a significant 24-h cycle for all emissions, suggesting a circadian rhythm of frequency fluctuation. [Work supported by the Knowles Research Foundation.]

R7. Some implications of nonlinear passive models of spontaneous otoacoustic emissions. C. Talmadge, A. Tubis (Physics Department, Purdue University, West Lafayette, IN 47907), and G. R. Long 
(Department of Audiology and Speech Sciences, Purdue University, West Lafayette, IN 47907)

The nonlinear passive cochlear model of $\mathrm{M}$. Furst and $\mathrm{M}$. Lapid [ J. Acoust. Soc. Am. 84, 222-229 (1988) ] has recently been criticized by Wit [J. Acoust. Soc. Am. 85, 2217 (1989)] on grounds that this model is not able to account for the observed statistical [Bialek and Wit, Phys. Lett. A 104, 173-178 (1984)] and external-tone entrainment [Long et al., Basic Issues of Hearing, edited by $\mathrm{H}$. Duifhuis et al. (Academic, London, 1988), pp. 93-100] properties of spontaneous otoacoustic emissions. The argument of Wit and the response to it by M. Furst [J. Acoust. Soc. Am. 85, 2218-2220 (1989)] are critically examined. On the basis of numerical simulations and exact solutions [Caughey and Payne, Int. J. Non-Linear Mech. 2, 125-151 (1967)] of the Fokker-Planck equation for stochastically driven nonlinear passive oscillators, it is concluded, in agreement with Wit, that nonlinear passive cochlear models of the type used by Furst and Lapid are not able to adequately deseribe the observed behavior of spontaneous otoacoustic emissions. [Work supported by NIDCD.]
R8. Parameter estimation for a resonant tectorial membrane cochlear model. J. B. Allen (Acoustics Research Department, AT\&'T Bell Laboratories, 600 Mountain Avenue, Murray Hill, NJ 07974)

One of the basic problems of cochlear modeling is in systematically determining the parameters of the model from neural data. Frequency domain models give the response along the basilar membrane for puretone inputs, whereas the data that are desirable to match are families of tuning curves as a function of frequency for many positions along the basilar membrane. This computational problem has been sidestepped by transforming the neural tuning curve data to place, forming neural excitation patterns. This allows for a major simplification of the data fitting process. If the neural data are accurately fit in the place domain for several well-separated frequencies, then the frequency tuning data should fit as well. Using this approach, fits to neural tuning curves using passive cochlear models were greatly improved over the entire range of CFs. The model assumptions will be discussed and the results will be compared to measured data.

\section{0:00-10:15}

\section{0:15}

R9. The effect of induced round window fistula on the SP/AP amplitude ratio. Kathleen C. M. Campbell (Department of Surgery, Southern Illinois University School of Medicine, Box 19230, Springfield, IL 62794), Michael M. Savage, and Lee A. Harker (Department of Otolaryngology, University of Iowa, Iowa City, IA 52242)

The ratio of the summating potential (SP) and action potential (AP) amplitudes was studied in 19 guinea pigs before and after surgical induction of a round window fistula. Stimuli included 2000 - and $8000-\mathrm{Hz}$ tone bursts and click stimuli at intensity levels of 100,90 , and $80 \mathrm{~dB}$ SPL. The following $p$ values are for the 90-dB SPL level, but results were similar for all three intensity levels. After fistula induction, the SP/AP ratio enlarged in response to the $8000-\mathrm{Hz}$ tone-burst $(p<0.0076)$ and click stimuli $(p<0.0001)$, but not for the $2000-\mathrm{Hz}$ tone-burst stimuli $(p<0.5627)$. Analyzed separately, the SP significantly enlarged after fistula induction for clicks $(p<0.0001)$ and $8000-\mathrm{Hz}$ tone bursts $(p<0.0006)$ but not for the $2000-\mathrm{Hz}$ tone bursts $(p<0.5110)$. The AP enlarged slightly after fistula induction in response to the clicks $(p<0.0052)$ but not in response to the $8000-\mathrm{Hz}(p<0.1780)$ or the $2000-\mathrm{Hz}(p<0.1723)$ tone bursts. These results suggest that the SP/AP amplitude ratio may be sensitive to the presence of perilymphatic fistula with selected stimuli.

\section{0:30}

R10. Comparisons of between- and within-subject variability in repeatedmeasures auditory brain-stem responses (ABRs) in 10- to 12-year-old children. Robert F. Oyler and Judith L. Lauter (Department of Speech and Hearing Sciences, University of Arizona, Tucson, AZ 85721)

In reports to this Society, and in various publications [ J. L. Lauter and R. L. Loomis, Scand. Audiol. 15, 167-172 (1986); 17, 87-92 (1988) ], results of repeated-measures ABR testing in young adults have been described, indicating that the variability of peak parameters, such as latency and amplitude, provides information that absolute values of these parameters do not: contrasts in between- versus within-subject consistency, and by ear of stimulation. Nine 10- to 12-year-old boys were tested in four biweekly sessions, with five ABR waveforms collected in each session for monaural right, monaural left, and binaural clicks. Relative variability measures of ABR latencies reveal adultlike patterns based on nonadult values: (1) contrast in between- versus within-subject consistency; (2) peak differences; and ( 3 ) ear differences. There are also differences in within- versus between-session consistency. Preliminary comparisons with results of similar testing in 5- to 7-year-old children [J. M. LordMaes and J. L. Lauter, J. Acoust. Soc. Am. Suppl. 185 , S38 (1989) ] and adults suggest that ABR variability may be sensitive to auditory-system developmental changes that continue long after ABR absolute peak latencies have achieved adult values. [Work supported in part by AFOSR.]

\section{0:45}

R11. Forward masking of the frequency following response. A. K. Krishnan (Department of Communications, University of Pittsburgh, 117 Cathedral of Learning, Pittsburgh, PA 15260) and J. D. Durrant (Department of Otolaryngology, University of Pittsburgh, 117 Cathedral of Learning, Pittsburgh, PA 15260)

The frequency following response (FFR) reflects sustained neural activity within the brain stem, phase locked to the stimulus at low frequencies. Attempts at resolving the issue of the origin of FFR along the basilar membrane via the subtractive high-pass masking technique have been unsuccessful. A tone-on-tone forward masking paradigm is described as an effective alternative method for assessing the place specificity of FFR. Recordings were made between forehead and the ipsilateral mastoid using normal adult subjects. FFRs were elicited by a $500-\mathrm{Hz}$ tone burst with masking frequencies from $500-4000 \mathrm{~Hz}$ presented at $10 \mathrm{~ms}$ before the probe. Results indicate that the FFR $(500 \mathrm{~Hz})$ was most affected by the $1000-\mathrm{Hz}$ masker, with less masking at $500 \mathrm{~Hz}$ and little or no effects at frequencies above $1000 \mathrm{~Hz}$. Additionally, some subjects exhibited an enhancement of the FFR for maskers about $1000 \mathrm{~Hz}$. Results suggest that our method is effective in evaluating frequency specificity of the FFR. A serendipitous finding revealed a possible means for discriminating between what might be separate on-set and sustained components of the FFR.

\section{1:00}

R12. The representation of synthetic stop consonants in a computational model of the dorsal cochlear nucleus. Michael J. Pont and Robert I. Damper (Department of Electronics and Computer Science, University of Southampton, Highfield, Southampton SO9 $5 \mathrm{NH}$ England)

A computational model of part of the mammalian auditory nervous system is described. This consists of three scissile stages simulating afferent neural processes within the cochlea, auditory nerve, and dorsal coch- 
lear nucleus (DCN). The model derives its input from a 128-channel cochlear filter bank. The artificial neuron units employed are simple encoder models whose responses and interconnections follow closely those reported in recent anatomical and physiological studies. The activity of the low-frequency DCN projection units in the model to three (labial, alveolar, and velar) synthetic voice onset time (VOT) stimulus series is shown to mimic the behavioral responses of human and chinchilla subjects [P. K. Kuhl and J. D. Miller, J. Acoust. Soc. Am. 63, 905-917 (1978) ] to these stimuli. The results suggest that DCN processing may have an important role in the perception of voicing contrasts. [Work supported by SERC and NICHD Contract N01-HD-5-2910.]

\section{1:15}

R13. Cyclic temporal pattern perception and neuronal adaptation. Donald A. Robin, Paul J. Abbas, and Linda N. Hug (Department of Speech Pathology and Audiology, The University of Iowa, Iowa City, IA 52242)

Sets of regularly repeating auditory stimuli elicit unique perceptions; listeners are able to identify specific temporal patterns. Some temporal patterns are unambiguous (only one pattern can be perceived), while others are ambiguous (numerous patterns can be detected). While the psychophysical properties of such percepts have been well studied, little is known about the underlying neurological bases of temporal pattern perception. In this experiment, the role of adaptation in temporal pattern perception was examined by studying neural responses in four cats to a temporal pattern that is perceptually unambiguous and one that is perceptually ambiguous. Measurements were made of the whole-nerve action potential, the auditory brain-stem response, and potentials from the surface of the primary auditory cortex. The adaptation patterns corresponded with the perceptual organization of temporal patterns in humans at all levels of the nervous system studied.

\section{1:30}

R14. Functional organization of normal human auditory central nervous systems observed with multiple noninvasive techniques: Year one of the CNS project. Judith L. Lauter (Department of Speech and Hearing Sciences, University of Arizona, Tucson, AZ 85721)

The coordinated noninvasive studies (CNS) project is designed to bring together a variety of noninvasive methods for studying living brains in order to demonstrate the feasibility of a "human neuroscience" paradigm based on a combination of behavioral testing with noninvasive neuroanatomical and neurophysiological examinations of the same individuals. Initial project focus is on brain asymmetries related to complex-sound processing. Preliminary results on subjects studied to date will be presented, documenting: (1) behavioral asymmetries expressed in terms of relative ear advantages, (2) individual differences in anatomical (MRI) asymmetries, (3) patterns of physiological "resting asymmetries," and processing asymmetries observed during auditory stimulation (qEEG, PET), (4) the degree of within-subject consistency of asymmetry direction and magnitude observed with several noninvasive methods (MRI, EPs, qEEG, etc. ), and (5) the coincidence of auditory-system asymmetry descriptions based on behavioral versus neuroanatomico-physiological measurements. [Work supported by AFOSR.]

\section{1:45}

R15. Perception of sequenced pitch and duration changes by patients with frontoparietal lesions. Karen A. Colson (Hearing and Speech Department, University of Kansas Medical Center, Kansas City, KS 66 103), Donald A. Robin, and Erich S. Luschei (Department of Speech Pathology and Audiology, University of Iowa, Iowa City, IA 52242)

Current evidence suggests that auditory processing of nonlinguistic pitch and timing features may be differentially impaired following focal, unilateral cortical insult. Three experiments were conducted to examine the ability of patients with well-defined, left, frontoparietal lesions and normal, matched controls to perceive relative pitch and/or duration changes in tonal sequences. Performance patterns for two right frontoparietal patients also were examined. Subjects identified whether or not any tone in a three-tone sequence was higher in pitch and/or longer than the other tones. Compared to the controls, the left anterior group typically required a significantly larger difference (e.g., $\mathrm{Hz}$ and/or $\mathrm{ms}$ ) between the standard and comparison tones to accurately identify relative changes in pitch, duration, and pitch and duration combined. The right frontoparietal patients performed the three tasks at a level similar to that of the normal controls. Based on the findings, the perception tasks seem to involve a complex interaction between temporal and spectral features and appear to be sensitive for detecting left, anterior patients' difficulties with these aspects of auditory processing. 


\title{
Session S. Speech Communication IV: Memorial Session for Dennis Klatt
}

\author{
Stefanie Shattuck-Hufnagel, Cochairman \\ Research Laboratory of Electronics, Room 36-511, Massachusetts Institute of Technology, Cambridge, Massachusetts \\ 02139
}

\author{
Kenneth N. Stevens, Cochairman \\ Research Laboratory of Electronics, Room 36-517, Massachusetts Institute of Technology, Cambridge, Massachusetts \\ 02139
}

\section{Ralph N. Ohde, Cochairman}

Vanderbilt University School of Medicine, Division of Hearing and Speech Science, Box 562, Station 17, Nashville,

Tennessee 37232

\section{Invited Papers}

\section{$8: 15$}

S1. Acousties and synthesis of fricative consonants. Kenneth N. Stevens (Research Laboratory of Electronics and Department of Electrical Engineering and Computer Science, Massachusetts Institute of Technology, Cambridge, MA 02139)

This paper attempts to integrate some recent research of Dennis Klatt on the analysis and synthesis of fricative consonants with further experimental and theoretical studies of fricative production. The speech production studies examine data on airflows and pressures during voiced and voiceless fricatives, and estimate from these data the time variation of the areas of the glottal and supraglottal constrictions and the spect ra of the sound sources at these constrictions. These calculations are based on theoretical and experimental data on airflow in constricted tubes and on sound generation in turbulent fow. Acoustic spectra in a number of utterances containing fricatives in various vowel environments are measured at critical points within the utterances, and are interpreted in terms of the production studies. Particular attention is paid to events near the consonant-vowel and vowel-consonant boundaries, where the dominant source changes from frication noise to aspiration noise to glottal vibration. Based on this research, new synthesis rules for fricative consonants are proposed. [Research supported in part by NIH Grant No. NS-04332.]

$8: 40$

S2. Dennis Klatt's contribution to automatic speech recognition. Victor W. Zue (Room NE 43-601 A, Spoken Language Systems Group, Laboratory for Computer Science, Massachusetts Institute of Technology, Cambridge, MA 02139)

Over the past 20 years, Dennis Klatt has made enormous contributions to the field of automatic speech recognition through his research, writing, and student supervision. In the early $70^{\circ} \mathrm{s}$, he served as a member of the steering committee of the ARPA Speech Understanding Research (SUR) program, providing leadership and guidance to the research community. He also participated actively in speech recognition research, first performing a set of spectrogram reading experiments assessing the role of various sources of knowledge, and later investigating the use of synthesis-by-rule techniques for word verification. Out of this involvement with the ARPA-SUR program came the landmark paper reviewing its technical achievement, as well as several publications describing his own proposals, LAFS and SCRIBER, for human and machine speech recognition. Over the past 10 years, Dennis directed his attention to the design of signal representation front-ends, as well as the investigation of perceptually motivated distance metrics in order to implement his speech recognition models. This talk pays tribute to Dennis's incredibly active research life by examining the legacy he left behind in speech recognition.

S3. Duration models and segmental quality in a text-to-speech system. Rolf Carlson and Björn Granström (Department of Speech Communication and Music Acoustics, Royal Institute of Technology, Box 70014, S-100 44 Stockholm, Sweden)

We have had the privilege of working together with Dennis Klatt for many years. In our presentation we will refer to some of Klatt's work that has had an infuence on our own work. Modeling of segmental duration 
was a central part in Klatt's work during the 1970s. This work resulted in a duration model in 1979 that captures many of the basic effects found in speech. This model has been used as a framework in the KTH textto-speech system. The use of quantity in Swedish demands expansions of the model. The Swedish study has been done in the context of a speech database from different speakers reading different text materials. In Klattalk, Klatt [J. Acoust. Soc. Am. 82, 737-793 (1987)] addressed all levels in a text-to-speech system, but special effort was placed on a general improved segmental quality. The quality of the best speech synthesis is, however, still far from that of human speech. Some recent efforts to improve the segmental intelligibility in our system will be described. This includes experiments with new synthesis strategies with an emphasis on modeling contextual variability. Analysis and synthesis of positional variants of the Swedish consonants are reported, and new strategies for synthesis are discussed. Based on analysis of the speech database, consonant rules affecting both source and resonator features were formulated and tested. Special efforts were made to handle the realization of consonant clusters. In this development work, diagnostic tests were used at regular intervals. Results from the last years' evaluation will be reported and discussed.

S4. Perceptual evaluation of MITalk and DECtalk. David B. Pisoni (Speech Research Laboratory, Department of Psychology, Indiana University, Bloomington, IN 47405)

In the spring of 1979 , we began the first of what would eventually become many dozens of behavorial studies on the perceptual evaluation of synthetic speech produced automatically by rule. The MITalk text-tospeech system was just nearing completion at MIT. At that time, I spent many hours with Dennis Klatt talking about and planning a variety of perceptual tests to evaluate the MITalk system. In this paper, I will first summarize the initial results obtained with the MITalk system in 1979. Then I will describe the evaluation of DECtalk. I believe that one of the reasons that DECtalk has consistently shown such high levels of segmental intelligibility, levels often approaching those observed with natural speech, was Dennis' intense fascination with the results of our error analyses of the MRT that he used to selectively modify, refine, and improve the quality of the synthetic speech produced by DECtalk. In working with Dennis, it became clear to me that one of his major goals was to develop a system tht would produce the very best quality synthetic speech possible. The data from additional perceptual tests demonstrate clearly that Dennis was successful in achieving his goals for DECtalk. DECtalk remains the standard against which all other text-to-speech systems are compared. [Work supported by NSF.]

S5. Adults and infants show a "prototype effect" for speech sounds. Patricia K. Kuhl (Department of Speech and Hearing Sciences, University of Washington, Seattle, WA 98195)

Dennis Klatt provided valuable assistance to many speech perception researchers. His advice on the synthesis of speech signals was particularly helpful, and investigators relied on his expertise when problems arose in the development of stimuli designed to approach a problem in a new way. This was the case in the preparation of stimuli to test whether there are "prototypes" for vowel sounds. The experimental question was whether or not adults and infants responded differently in a within-category vowel discrimination task when the "standard" stimulus was an exceptionally good instance of the vowel $/ \mathrm{i} /$-a prototype of the category -as opposed to a nonprototypic / $\mathrm{i} /$ vowel. The results showed that when the prototype of the category served as the standard stimulus, it was more difficult to hear differences between it and novel / $/$ / vowels than it was to hear differences between the nonprototypic stimulus and novel /i/ vowels. In other words, the prototype was perceived to be more similar to new members of the category than was the nonprototypic stimulus. The effect was observed both in adults and in 6-month-old infants. Described first will be the hypothesis underlying the prototype test, the method used to construct the stimuli (the test stimuli for the prototype and the nonprototype were scaled psychophysically using the "mel" scale), and the results on English adults and infants. Described next will be the second phase of the research program, which entails cross-language tests on Swedish adults and infants. The cross-language tests are designed to assess two different explanations for the prototype effect: (1) that particular vowel stimuli $(/ \mathrm{a}, \mathrm{i}, \mathrm{u} /)$ are inherently more resistent to the effects of articulatory/ acoustic change (Stevens' quantal theory) and (2) that the effects observed in American adults and infants are attributable to experience in listening to English, even in the first 6 months of life. Careful preparation of the English and Swedish stimuli was critical in designing these experiments. Dennis Klatt was always ready to provide advice on such matters; his assistance is gratefully acknowledged and sorely missed. [Work supported by NIH.1

\section{0:20-10:35 \\ Break}

\section{Contributed Papers}

10:35

S6. Perceptual and acoustic charactertics of distorted $/ r /$ Ralph N. Ohde, Michael E. McCarver (Division of Hearing and Speech Sciences, Vanderbilt University School of Medicine, Nashville, TN 37232), and Donald J. Sharf (Boca Raton, FL 33496)
One type of articulation disorder is a sound distortion that has been defined as an allophonic variation within the perceptual boundary of a target phoneme. An established finding in speech perception is that sounds are more accurately identified across sound categories than within sound categories. In order to determine if distorted $/ \mathrm{r} /$ could be accurately and reliably perceived, six speech pathologists identified the produc- 
tions of prevocalic $/ r /$ and / w/ words of 12 children diagnosed as having an $/ r /$ misarticulation. The results of the identification tests revealed a relatively high average distorted $/ \mathrm{r} /$ category of $70 \%$ or better for four children. Moreover, intrasubject reliability scores for these distorted $/ \mathrm{r} /$ children averaged $80 \%$ or better. Preliminary findings of spectrographic analyses of formant transition onsets show that $F 3$ onsets of distorted $/ r /$ are substantially higher than $F 3$ onsets of $/ \mathrm{r}$ / for normal and synthetic versions of children's speech. [Work supported by Biomedical Research Support Grant No. RR-05424.]

\section{0:47}

S7. Stress shift as the placement of phrase-level pitch markers Stefanie Shattuck-Hufnagel (36-511 Massachusetts Institute of Technology Research Laboratory of Electronics, 77 Massachusetts Avenue, Cambridge, MA 02139)

The $F 0$ contours produced by the text-to-speech conversion program Klatttalk [D. H. Klatt, J. Acoust. Soc. Am. 82, 737-793 (1987)] are based on a translation [S. Maeda, RLE-QPR 114, 193-211 (1974)] for American English of the "hat pattern" approach developed for Dutch [ $J$. 't Hart and A. Cohen, J. Phon. 1, 309-327 (1973) ]; this approach is similar to an earlier description by Mattingly [I. G. Mattingly, Supplement to Haskins Laboratory Status Report on Speech Research, 1-223 (1968) ]. One question that arises for this view of $F 0$ patterns is how might it deal with the phenomenon of "stress shift": For some speakers, the prosodic prominence on the main-stress syllable of words like "thirteen" and "Mississippi" is perceived to move to an earlier syllable when these words appear in phrases like "thirteen men" and "Mississippi mud." This paper will report pitch and duration measurements designed to evaluate the hypothesis that at least some aspects of the stress shift phenomenon can be described as the simple placement of the onset rise of a hat pattern on an early syllable of the prosodic phrase.

\section{0:59}

S8. Synthetic speech audiometry, Corine Bickley (Sensimetrics Corporation, Building 100, One Kendall Square, Cambridge, MA 02 139) and Gerald Kidd (Department of Communication Disorders, Boston University, Boston, MA 02215)

A new hearing test is being developed that is based on presenting to listeners sets of synthesized words with well-defined acoustics properties. The test is based in part on work by Gòsy et al. [Proc. 1] th ICPS, Tallinn (1987) ], and its aim is to estimate a listener's hearing sensitivity from errors in word discrimination. Sets of words have been synthesized (using the Klatt synthesizer) that differ from each other by one of two phonemes (e.g., sit sat fat fit). The synthesis was guided by two goals. (1) Each word should differ from another word in a set by only one acoustic feature; the primary difference must be limited to a specific frequency band (e.g., sit versus sat differ by the frequency of the first formant). (2) The synthesized words should be highly intelligible to normally hearing listeners in a quiet environment. Initial results were obtained by presenting the synthesized words combined with white noise of various levels in a forced-choice paradigm to normally hearing subjects,. As expected, word discriminability was correlated with the salience of the acoustic feature that distinguishes the word relative to the added noise. The feasibility of using synthesized word sets of this type to detect and estimate the severity of hearing impairment will be discussed. [Work supported by a grant from NIH.]

\section{1:11}

S9. Acoustic properties of $/ h /$. Sharon $Y$. Manuel and Kenneth N. Stevens (Research Laboratory of Electronics, Massachusetts Institute of Technology, Cambridge, MA 02139)

The aim of this paper is twofold: (1) to investigate the physical mechanisms of sound generation for the consonant $/ \mathrm{h} /$ and (2) to examine the timing of supraglottal and glottal movements of $/ \mathrm{h} /$. Utterances in which $/ \mathrm{h} /$ was present or absent (e.g., "new heart" versus "new art") were analyzed acoustically and contrasted. The corpus consisted of about 20 such utterances repeated several times by three speakers. The acoustic data showed evidence of breathy voicing at the $/ \mathrm{h} /$-vowel boundary in all cases, and that generation of turbulence noise during the consonant occurred both in the vicinity of the glottis (aspiration noise) and the vicinity of the supraglottal constriction (frication noise). The relative contribution of the two noise sources depended on the vowel, with greater frication noise occurring for high vowels. When an $/ \mathrm{h} /$ was in position between two vowels or glides, it generally added little or no duration to the utterance, relative to the contrasting utterance with no $/ \mathrm{h} /$. Implications for the phonological status of $/ \mathrm{h} /$ as discussed. [ Work supported by NIH grants to MIT.]

\section{1:23}

S10. Perception of some consonant contrasts in noise. Abeer Alwan (Research Laboratory of Electronics and the Department of Electrical Engineering and Computer Science, Room 36-511, Massachusetts Institute of Technology, Cambridge, MA 02139)

The goal of the present study is to examine the acoustic properties that listeners use to distinguish between speech sounds when these sounds are presented in noise. A series of perceptual experiments was conducted using natural stimuli consisting of nonsense $\mathrm{CV}$ syllables, where $\mathrm{C}$ was either $/ \mathrm{m} /, / \mathrm{n} /, / \mathrm{b} /$, or $/ \mathrm{d} /$, and $\mathrm{V}$ was either $/ \mathrm{d} /, / \mathrm{i}^{\mathrm{y}} / /$, or $/ \mathrm{o}^{\mathrm{w}} /$. The stimuli were degraded by adding various levels of white noise and were presented to subjects in identification tests. Preliminary results show that when the noise is at a level such that the transition of the second formant frequency of the vowel is masked, confusions between the place of articulation for the stimuli occur. Noise levels for which confusions in manner of articulation occur can also be predicted from masking theory. These results are compared with results reported earlier [e.g., Miller and Nicely (1954) ] where the thresholds of idenfication were described in terms of the signal-to-noise ratio. These results will be discussed further in terms of the acoustic theory of speech production and the masking theory of the auditory system. [Work supported in part by an NIH grant.]

\section{1:35}

S11. The influence of selected acoustic cues on the perception of $/ 1 /$ and /w/. Carol Y. Espy-Wilson (Research Laboratory of Electronics, Massachusetts Institute of Technology, Cambridge, MA 02139)

In a semivowel recognition system developed by Espy-Wilson [Mass. Inst. Technol. Res. Lab. Electron. Tech. Rep. No. 531 (1987) ], the sounds $/ 1$ and $/ w /$ were frequently confused, especially when they occurred intervocalically. In this study, the perceptual importance of some of the cues used in the recognition system, as well as some others which appear to be salient, were investigated. An [ala]-[awa] continuum was synthesized. The starting point was an easily identifiable [ala] stimulus. Three factors were varied orthogonally to shift the percept towards [awa]: (1) the rate of change in the formant transitions between the semivowel and following vowel, (2) the rate of change in the amplitudes of $F 3, F 4$, and $F 5$ between the semivowel and following vowel, and (3) the spectral shape of the semivowel (coronal or labial). Preliminary results of an identification test show that spectral shape and the rate of change of the formant transitions are important cues, whereas the rate of change in the amplitudes of $F 3, F 4$, and $F 5$ appears to have a negligible effect on listeners' responses. For example, with formant transitions of $30 \mathrm{~ms}$ or less, [ala] is heard. With formant transitions greater than $40 \mathrm{~ms}$, the perception moves towards [awa]. The results also show that a few listeners had difficulty hearing [awa] when the semivowel had a coronal shape, despite the rate of change in the formant transitions being biased towards / w/. These results will be discussed with respect to past and future research in speech recognition. 
11:47

S12. Combining statistical and linguistic models for synthesis of prosodic contours. Mari Ostendorf (Boston University, ECS Department, Boston, MA 02215), Patti Price (SRI International, Menlo Park, CA 94025), Stefanie Shattuck-Hufnagel (Massachusetts Institute of Technology, Cambridge, MA 02139), Nanette Veilleux, Colin Wightman, and Rudy Garcia (Boston University, Boston, MA 02215)

"It is very important to get the timing, intonation, and allophonic detail correct in order that a sentence sound intelligible and moderately natural." [D. Klatt, J. Acoust. Soc. Am. 82, 737-793 (1987)]. This important review article included prosody as a research issue for improving text-to-speech synthesis. Klatt's suggestions for improving prosody are addressed here: Development of new systems for control of $F 0$ and duration, and mechanisms for adding variety. The proposed synthesis system is a statistical model trained on text, parts of speech, pronunciation, lexical stress, prosodic labels (major and minor boundaries, accents, etc.), and acoustic parameters (relative $\boldsymbol{F} 0$ and duration). The synthesis problem is to predict the prosodic labels and acoustic parameters given the text and the statistical model. Several hours of speech have been collected from professional FM newscasters, a labeling scheme has been converged on, and a portion of the data has been labeled. The components of the system so far implemented will be discussed: (1) statistical modeling of sequences of parts of speech to predict major prosodic breaks, (2) the role of breath noise in naturalness, and (3) the implementation of a sinusoidal model for duration and pitch modification of waveforms. [Work supported by NSF.]

WEDNESDAY MORNING, 29 NOVEMBER 1989

DIRECTORS' ROW 43, 8:30 A.M. TO 12:00 NOON

\title{
Session T. Structural Acoustics and Vibration II: Characterization of Viscoelastic Polymers
}

\author{
Wayne T. Reader, Chairman \\ Vector Research Company, Inc., 6903 Rockledge Drive, Suite 1200, Bethesda, Maryland 20817
}

Chairman's Introduction-8:30

Invited Papers

8:35

T1. Measurement of polymer complex modulus properties using several techniques. Thomas $M$. Lewis (Anatrol Corporation, 10895 Indeco Drive, Cincinnati, OH 45241) and Dominique Legros (Metravib Instruments, France)

Various techniques for measurement of the dynamic mechanical properties of polymers are discussed. Special emphasis is given to the vibrating beam, SDOF resonance, SDOF impedance, creep, relaxation, forced oscillation (Metravib viscoanalyzer), and forced torsional oscillation (Metravib micromechanalyzer) techniques. Material property data, generated over wide ranges of temperature and frequency (using the above techniques), are presented in terms of reduced frequency upon application of an Arrhenius temperaturefrequency shift function. Comparison of these properties in the reduced frequency format indicates good correlation between techniques. Specific examples are included consisting of data generated from commercially available materials in both shear and tension-compression states of stress from which estimates of Poisson's ratio may be determined.

T2. The ASTM E-756 Damping Standard-The good, the dangerous, and the pitfalls. Michael L. Drake (University of Dayton, 300 College Park JPC-36, Dayton, OH 45469)

The resonant cantilever beam test procedure is a reliable test method from which complex modulus data can be determined. The ASTM E-756-83 Standard was developed around this test procedure. This test method has been used since Oberst started work in the early 1950s. Ross, Kerwin, and Ungar expanded the usefulness of the test through the development of sandwich equations. Although the test procedure is reliable, it was developed when the fundamental interest in complex modulus data was focused on the transition region. As a result, when users begin to stretch the viscoelastic properties testing further into the rubbery and glassy regions problems developed. This paper will detail the idiosyncrasies of this test method and the analytical equations used to calculate the complex modulus data from the raw test data. It will be demonstrated that apparently good, self-consistent data can be very inaccurate. The effects of test specimen configuration and the modulus of the viscoelastic material on the accuracy of the complex modulus data and the utility of the various test specimens will be discussed. The commonality of the problems in the best test with other popular test methods will also be discussed and suggested test parameters will be given to enhance complex modulus data accuracy. 
T3. Frequency-temperature superposition in polymer damping behavior. David I. G. Jones (Materials Laboratory, WRDC/MLLN, Wright-Patterson AFB, OH 45433)

The problem of determining optimum shift factors for frequency-temperature dependence of polymeric material complex modulus properties has not been fully resolved even half a century after the pioneering work of Williams, Landel, and Ferry. The question of what form, such as the WLF (Williams, Landel, Ferry) equation or the Arrhenius equation, best depends on both the material and the quality of the test data. In most cases, the data scatter is sufficient to prevent a definitive choice. These issues will be discussed with reference to available test data for several polymeric materials, obtained by various measurement techniques. It will be shown that the least ambiguous shift factor estimates are obtained when data scatter is as low as possible, and the frequency range of data at each temperature is as wide as possible, as would be expected. It will also be shown that differences arising from the use of different shift factor equations are not sufficient for most engineering applications.

\section{9:50}

T4. Presentation and modeling of complex modulus. Lynn Rogers (WRDC/FIBG, Area B, Building 24C, Room 220, Wright-Patterson AFB, OH 45433-6503)

One of the historical challenges in the field of passive vibration damping technology is the modeling of experimental complex modulus data as a function of temperature and frequency. Several models exist for the temperature shift function (TSF); i.e., Arrhenius, WLF, exponential, etc. Several models of complex modulus as a function of reduced frequency also exist. All existing TSF and CM models fail to represent at least some sets of data with desired accuracy and efficiency. Consider a linear, constant coefficient, stable system, and its frequency response function. It is well known that if the real component of the complex-valued frequency response function is given over the infinite frequency range, then the imaginary component may be obtained. The complex modulus of vibration damping materials is such a system. Extensive work with fractional calculus based models for complex modulus has established their viability and potential attractiveness. A ratio of factored polynomials of one-half order is proposed to model the complex modulus. This CM model is attractive from a number of viewpoints: The proper interrelationship of the real and imaginary components is guaranteed; an adequately large number of terms may be used in order to accurately model the complex modulus; an expression may be developed for the real component that lends itself to fitting data by collocating through a number of points; closed-form expressions may be developed for compliance, relaxation modulus, and creep compliance which also lend themselves to collection fitting of experimental data, etc. With modern computational power, this model becomes both accurate and efficient. Previous work has established the slope of the TSF as the characteristic which causes complex modulus data to be properly shifted; therefore, a new approach to modeling the TSF is proposed. The new model is based on determining values of slopes at equally spaced temperatures, fitting a cubic spline through these points (i.e., knots), storing the coefficients, and integrating the cubic spline analytically. The concept of reduced temperature is introduced, used as a convenience for the present effort and proposed as an additional method of presenting data in a form useful to the damping industry. The core of the revolutionary concept is using the simultaneous modeling of both real and imaginary components as the criteria to enable the set of data to establish its TSF. Previous techniques have used real modulus, imaginary modulus, and loss factor as a function or reduced frequency, sometimes in a least-squares sense, and sometimes visually, as the criteria. The above CM and TSF models are essential to the iteration strategy required to determine parameter values for both models. The iteration scheme is conceptually straightforward. Approximations to the CM and to the TSF are obtained. For each TSF knot, the reduced temperature is used to determine the associated reduced frequency, the current loss factor curve is compared to the corresponding experimental value and the value of the slope adjusted accordingly, the real component collocated for the updated TSF, etc. Examples are given and discussed.

\section{0:15}

T5. Design of polymers for viscoelastic damping applications. R. E. Wetton (Polymers Laboratories, Ltd., The Technology Center Epinal Way, Loughborough LE11 OQE, United Kingdom) and J. L. Duncan (Polymers Laboratories, Inc., Amherst Fields Research Park, 160 Old Farm Road, Amherst, MA 01002)

The large frequency dependence of the dynamic moduli of polymers allows the design of materials with special damping and engineering applications. The temperature dependence of these properties is, however, a drawback in many cases. The present paper reviews the changes of Youngs, shear, and bulk moduli with frequency and temperature. Their accurate measurement and predictions via time/temperature superposition methods are discussed, as are the relationships between these parameters with changing temperature. The design of elastomers with high damping and minimized temperature variation is discussed with some examples.

\section{0:40}

T6. Internal friction in polymer systems. Jozef Bicerano and James K. Rieke (Materials Science and Development Laboratory, Central Research, The Dow Chemical Company, Midland, MI 48674)

Internal friction is an effective mechanism for dissipating energy in polymer systems. Of particular interest is the ability to dampen and absorb unwanted acoustic and mechanical vibrations. The use of internal friction 
will become more effective when detailed geometric, thermodynamic, and kinetic models are developed for the physical phenomena producing the vibration damping. A model that relates the viscoelastic properties of polymeric systems to their molecular level structures has been developed. This model considers the nature of the temperature and frequency dependences of the storage and loss components of the complex moduli of polymers. It provides correct and internally consistent correlations. Therefore, it constitutes a first step in an attempt to bridge the gap between the results of application of an external stress (such as acoustic or mechanical vibration) and the molecular level properties of the polymers.

\title{
$11 ; 05$
}

T7. Characterizing viscoelastic materials using the free volume microprobe. Bret $A$. Mayo, James $P$. Pfau, and Duryodhan Mangaraj (Battelle Memorial Institute, 505 King Avenue, Columbus, OH 43201-1693)

A great deal of effort has been directed toward the development of new polymer blends and interpenetrating polymer networks in recent years. This approach has emerged as an important means of developing new polymeric materials and improving properties such as sound damping. The thermodynamic models that best describe polymer-polymer interactions have an important free volume contribution. Until recently, however, there was no technique that could make a direct, nondestructive measurement of the molecular free volume. Using the free volume microprobe (FVM), it is now possible to characterize both the average free volume site size and the relative number of free volume sites. Thus it is possible to make some assessment of the total free volume as well as the free volume distribution. The basic theory of the FVM technique will be presented as well as some supporting data from a series of miscible, immiscible, and partially miscible polymer blends.

\section{Session U. Underwater Acoustics III: Computational Acoustics I}

\author{
Henrik Schmidt, Chairman \\ Department of Ocean Engineering, Massachusetts Institute of Technology, Cambridge, Massachusetts 02139 \\ Chairman's Introduction-8:30
}

Invited Papers

8:35

U1. Inversion using simulated annealing. Atanu Basu and L. Neil Frazer (HIG, University of Hawaii Manoa, Honolulu, HI 96822)

The problem here is finding the profile $\bar{m}$ that minimizes $E(\bar{m})=\|\bar{d}-\bar{g}(\bar{m})\|-\epsilon\left\|\bar{m}-\bar{m}_{0}\right\|$ in which $\bar{d}$ is a data set, $\bar{m}_{0}$ is the bias, and $\bar{g}$ is a Green's function. If $\bar{g}$ is nonlinear and the dimension of $\bar{m}$ is large, then finding $E_{\min }$ is difficult. Simulated annealing (SA) seeks $E_{\min }$ by sampling from the probability distribution $p(\bar{m})=\exp [-E(\bar{m}) / T] / Z$ in which $T$ is so small that $p$ spikes at $E_{\min }$. This would be silly if one had to generate $Z$ numerically, but $S A$ does not need to know $Z$. The physical analogy for $S A$ is that each component of $\bar{m}$ is an atom in a melt having temperature $T$ and free energy $E$. Rapid cooling of the melt gives a glass (high $E$ ) but slow cooling gives a single crystal (low $E$ ). Problems with SA are that slow cooling consumes the computer budget faster than it finds $E_{\min }$ and that the freezing temperature $T_{c}$ is difficult to determine. Accordingly, much research on SA concerns cooling schedules that do not require $T_{c}$. In wave field inversion problems, it was found that known cooling schedules gave poor results and that knowledge of $T_{c}$ was essential. A rapid method to find $T_{\mathrm{r}}$ was discovered. Also, SA was no longer thought of as a process that terminates: rather $T_{c}$ is determined accurately keeping the melt at $T_{c}$ and letting SA find many good $\bar{m}$. A measure of resolution in $\bar{m}$ space is the "order parameter" $\eta=\|\bar{m}-\hat{m}\|$, where $\hat{m}$ is the unknown true profile. Expected values of $\eta(E)$ can be obtained from synthetic data. 
U2. Adaptive array processing of full-wave acoustic fields. Michael S. Wengrovitz (Atlantic Aerospace Electronics Corporation, Waltham, MA 02154) and Henrik Schmidt (Massachusetts Institute of Technology, Cambridge, MA 02139)

Adaptive array processing methods have been developed and applied in a number of areas including radar, communications, and underwater acoustics. Assessing the performance of these methods is more difficult in the underwater acoustics context due to the complex influence of the ocean environment on the signal and noise fields. Arrivals across the array are not plane waves, as typically assumed in radar and communications problems, but are solutions to the wave equation applicable to the particular ocean environment. This paper describes an efficient computational method that interfaces the full-wave solution for horizontally stratified media with adaptive array processing algorithms. The computational method has been used to provide coherent arrival data for full-wave array beam pattern calculation, and noise spatial-correlation matrices for deriving array gain corresponding to various adaptive arrays. The computational method has been used to study a number of important array processing issues for multidimensional arrays with large number of elements in oceanic environments. Example issues discussed in the paper include applicability of partially adaptive beamforming algorithms, sensitivity of array performance to environmental parameters such as bottom composition, convergence rates of adaptive nulling algorithms in underwater environments, and array calibration.

U3. Recent developments in parabolic equation modeling. Michael D. Collins (Naval Research Laboratory, Washington, DC 20375)

The parabolic equation (PE) is one of the most important computational models for underwater sound propagation. Since the PE was introduced into the field of underwater acoustics by $F$. D. Tappert, a great deal of research has been devoted to extending its capabilities, accuracy, and efficiency. In its present state, the PE is capable of handling three-dimensional problems, rough interfaces, and interaction with elastic ocean bottoms. A higher-order $\mathrm{PE}$ is accurate for arbitrarily large propagation angles. The rotated PE, which marches the solution parallel to the ocean bottom, provides improved accuracy for problems involving sloping bottoms. The efficiency of the finite-difference solution of the PE has been improved for range-dependent problems with a new algorithm for solving tridiagonal systems. The time-domain PE efficiently solves pulse propagation problems directly in the time domain. Some recent progress as well as some unresolved issues in PE modeling will be discussed.

\section{Contributed Papers}

9:35

U4. Parabolic equation starting field for a low-frequency source near an interface. Kenneth E. Gilbert and Dehua Huang (National Center for Physical Acoustics, University, MS 38677)

Experiments in underwater acoustics often use a low-frequency source close to the bottom. In such situations, the standard Gaussian starting field for parabolic equation calculations can grossly violate the boundary conditions at the water-bottom interface. In this paper, a simple operator method is derived that gives the correct boundary conditions in the starting field and also properly excites the "high-angle" modes and the continuum. As limiting cases, the standard Gaussian starting field of Brock [NORDA Tech. note 12, Naval Ocean Research and Development Activity, NSTL Station, MS (1978)] and the high-angle starting field of Greene [J. Acoust. Soc. Am. 76, 1764-1773 (1984) ] are obtained analytically. The method used here is compared with numerical results from a fast-field program for a low-frequency source near the bottom in shallow water.

9:50

U5. Optimal absorber potentials in PE modeling. F. J. Ryan and C. David Rees (Code 541, Naval Ocean Systems Center, San Diego, CA 92152-5000)

The parabolic equation (PE) approximation-essential in solving range-dependent propagation problems-relies on iterated fast Fourier transforms (FFTs). Preventing significant errors due to the periodicity of the finite FFT ("ringing") requires inserting an absorber potential to confine the wave energy to the range of interest. Such potentials, or sponges, have, to date, been strictly ad hoc. The problem of creating an optimal sponge by using an optical model analogy of the reflectionless potential $U=U_{\mathrm{t})}$ [ $[\operatorname{sech}(a * x)]^{2}$ of 1-D quantum mechanics is studied. This approach permits the development of closed-form expressions for both transmission and reflection coefficients in terms of elementary transcendental and gamma functions of complex argument. Using these coefficients and an efficient technique for evaluating such gamma functions yields a quantitative study of the problem of the optimal sponge. These results demonstrate that, for relevant parameter domains, there may be several "branches" of minimal paths in sponge parameter space, including one particularly well suited to the demands of PE modeling. [Work supported by NOSC-IR and OCNR.]

\section{0:05}

U6. Modal decomposition of fields in range-varying waveguides. David J. Thomson (Defence Research Establishment Pacific, FMO Victoria, British Columbia VOS 1B0, Canada)

Numerical solutions to parabolic equations (PE) provide the total contribution to the field at each point on the computational grid. For some applications, it is instructive to examine the propagation of individual spectral components, particularly in range-dependent waveguides, where mode coupling can be important. This paper presents a novel method for decomposing a numerical PE field into its horizontal wavenumber 
spectrum. At a given range in a range-dependent waveguide, the $P E$ field versus depth is provided as initial data to a PE model which is used to propagate the field in a range-independent waveguide characterized by the local environmental conditions at the range of the initial data. A Fourier transform of this propagated field yields the PE modal spectrum directly. Moreover, if the standard parabolic equation is used to propagate the field, then the PE modal spectrum can be postprocessed into the correct modal spectrum for the one-way wave equation [D. J. Thomson and D. H. Wood, J. Acoust. Soc. Am. 82, 224-232 (1987)]. A numerical example involving upslope propagation is used to illustrate the proposed spectral decomposition method.

\section{0:20}

U7. Boundary integral method for acoustic propagation in laterally inhomogenous medium. Woojae Seong and Henrik Schmidt (Massachusetts Institute of Technology, Cambridge, MA 02139)

A hybrid analytical-numerical method that combines boundary element and full-wave solution technique is presented for two-dimensional acoustic propagation in laterally inhomogenous and simultaneously vertically stratified medium. First, the range-dependent ocean is divided into range-independent sectors and the field within each sector is expressed by a boundary integral over the vertical sector boundaries in terms of a set of unknown boundary displacements. Proper choice of a set of distribution functions (here it is found to be Legendre polynomials) for these boundary displacements leads to efficient evaluation of the kernel of the boundary integral by means of global matrix approach, as implemented in the SAFARI code [H. Schmidt and F. B. Jensen, J. Acoust. Soc. Am. 77, 813-825 (1985)]. This leads to a matrix relation between the displacement and stress amplitudes along the vertical boundaries, similar to the stiffness matrix of finite element method. Once solved for the displacement amplitudes at the sector boundaries using a Galerkin approach, the boundary integral formulation directly yields the total field within each sector including both the forward propagating and reverberated field. A few examples of the canonical problems will be given to explore the feasibility and efficiency of the proposed new method for solving various range-dependent ocean acoustic propagation problems.

\section{0:35}

U8. The problem of energy conservation in one-way wave equations. Michael B. Porter, Carlo M. Ferla, and Finn B. Jensen (SACLANT Undersea Research Centre, 19026 La Spezia, Italy)

A recent attempt to define and numerically solve range-dependent benchmark problems in ocean acoustics [J. Acoust. Soc. Am. Suppl. 181 , S39-S40 (1987)] revealed some inherent problems with energy conservation for one-way wave equations when applied to sloping bottom environments. The problem turned out to be associated with the use of a stair-step approximation to a sloping interface, and, in particular, with the rise of the stair steps, where a one-way equation allows only one out of two necessary interface conditions to be fulfiled (continuity of pressure or horizontal particle velocity). The loss of energy increases with increasing slope and density contrast at the water/bottom interface, and it can become quite significant ( $3-6 \mathrm{~dB}$ ) for slopes of a few degrees and a density contrast of 2. A simple fix to this problem is to solve for a density-reduced pressure $(p / \sqrt{\rho})$ instead of pressure $(p)$, a trivial modification to existing one-way parabolic equation codes. Numerical results for both up- and downslope propagation show that the modified wave equation performs very well with errors being reduced by $75 \%$ for realistic slopes and density contrasts.

\section{$10: 50$}

U9. A convolution algorithm for standard parabolic-equation modeling. Robert F. Joyce and Martha E. M. Head (Naval Oceanographic Office, Stennis Space Center, MS 39522-5001)

The split-step Fourier algorithm for solving the acoustic parabolic equation involves two Fourier transforms, one forward and the other inverse. These may, of course, be replaced by a convolution. For the standard parabolic equation, the particular form of one of the convolved factors allows the convolution integral to be solved using a single Fourier transform. Application of this technique results in a limitation on the allowed range step. When applicable, however, the convolution algorithm reduces computer run times to about $60 \%$ of those required by the standard split-step Fourier algorithm. Examples are presented and limitations are discussed.

\section{1:05}

U10. Analysis of finite element and finite difference methods for the "parabolic" equation. Georgios D. Akrivis and Vassilios A. Dougalis (Department of Mathematics, University of Crete, Iraklion, Crete, Greece)

Some recent results of the authors on the construction and analysis of finite element and finite difference methods for the Schrödinger equation ( viewed as one-dimensional "parabolic" approximation to the Helmholtz equation in underwater acoustics) will be surveyed. The results include the construction, analysis, and implementation of efficient, conservative finite element methods of high order of accuracy based on spline approximations in the depth variable and suitable Padé-type techniques in the range. In addition, optimal error estimates for classical second-order accurate finite difference techniques of Crank-Nicolson type for the "parabolic" equation with interfaces and variable bottom will be presented.

\section{1:20}

U11. A marching method for some elliptic models of wave propagation. George H. Knightly and Donald F. St. Mary (Department of Mathematics, University of Massachusetts, Amherst, MA 01002)

Parabolic equation (PE) methods for wave propagation in the farfield are frequently obtained from elliptic equations valid in the farfield. Here, properties of the elliptic models are discussed directly. The equations are discretized and a marching method is obtained. Although the underlying initial value problems are not well posed, in certain parameter ranges, the marching method is stable when the step sizes are restricted in a suitable way. Under such restrictions, propagation loss curves are obtained that show good agreement with exact solutions of some test problems. Both fluid and elastic wave problems are considered. [Work supported in part by ONR.] 


\title{
Session V. Architectural Acoustics III: Acoustics of Teleconferencing Facilities
}

\author{
David L. Klepper, Chairman \\ Klepper Marshall King Associates, Ltd., 7 Holland Avenue, White Plains, New York 10603
}

Chairman's Introduction-8:35

\section{Invited Papers}

\section{$8: 40$}

V1. Teleconferencing-The acoustical environment and directional transducers. J. E. West and G. W. Elko (Acoustics Research Department, AT\&T Bell Laboratories, Murray Hill, NJ 07974)

The performance of teleconferencing systems are largely a function of the acoustical environment, the type, and the location of loudspeakers and microphones. Acoustical treatment in most conference rooms is limited to carpeted floors and an acoustical ceiling. For the most part, present microphones are designed to work best under close talking conditions and have omnidirectional, or first-order unidirectional characteristics. Two small experimental conference rooms have been constructed in which the effects of room parameters such as reverberation time, noise level, and discrete echo control can be independently studied. Broadband ( $8-\mathrm{kHz})$ directional microphones and steerable directional arrays, mostly of second order, are being investigated for a variety of acoustical environmental conditions. The easily adjustable conference rooms allow us to investigate these microphone systems with respect to their directional properties, placement, and steering algorithms. Loudspeaker directional characteristics, and advantages of stereo transmission are also under investigation. The experimental conference rooms and new directional microphones will be described and a discussion of trade-offs between major parameters for speech reception and transmission will be given.

\section{9:10}

V2. Microphones and feedback suppression in teleconferencing. Michael Petterson and Steve Julstrom (Shure Brothers, Incorporated, 222 Hartrey Avenue, Evanston, IL 60202-3696)

The effect of room acoustics on microphone placement and selection for teleconferencing systems and current approaches to electroacoustic feedback and echo control in teleconferencing systems will be discussed.

\section{9:40}

V3. Teleconference room acoustics. R. Kring Herbert (Ostergaard Associates, 115 Bloomfield Avenue, Caldwell, NJ 07006)

In dedicated teleconference rooms, there is an acoustical conflict between finishes for optimum microphone pick-up and those for optimum speech intelligibility within the space. This paper looks at the implications of this for teleconference room finishes and geometries as well as other acoustically critical factors such as sound isolation and ambient noise control. Recommendations are given for optimum performance.

V4. Room acoustic and sound system design for full duplex teleconference facilities using ceiling-mounted transducers. Wade R. Bray (Jaffe Acoustics, Inc., 114-A Washington Street, Norwalk, CT 06854)

Room acoustics and the effects of differing conditions on performance of a full-duplex audio teleconference system, which nulls direct-path coupling, leaving only coupling by reflected energy, were studied. Room impulse responses and signal-to-noise ratios at talker locations and at transducer locations were taken for rooms with differing acoustic conditions, as were binaural recordings of the impulse response tests and of sample teleconferences in the same situations, for subjective comparison and simultaneous analysis. Long working distances from talkers to microphones offer the advantage to users of freedom of placement and movement, but at the cost of strict limits on ambient noise loudness and, more particularly, noise structure (annoyance components). Early reflections within the psychoacoustic fusion time limit described by the Haas effect were found beneficial, while reflections beyond that time proved detrimental both to intelligibility and to circuit function. Discrete energetic late reflections are especially undesirable. Optimization for various room configurations was studied, and criteria presented for obtaining subjectively high-quality teleconferences between rooms and from rooms to handsets. 
V5. Employing the complete acoustical palette in teleconferencing design. Peter D'Antonio (RPG Diffusor Systems, Inc., 12003 Wimbleton Street, Largo, MD 20772)

The prevailing architectural acoustics approach in teleconferencing is "Deader is better" or "No acoustics is better than bad acoustics." In some situations, this is appropriate, but there are alternative approaches to reflection control in teleconferencing facilities which make use of the entire acoustical palette, namely absorption, reflection, and diffusion. Since the application of diffusive surfaces is new to the teleconferencing environment, a review of the development and the spatial, temporal, and spectral properties will be presented. A teleconferencing design for a board room will be proposed that makes use of an integrated suspended ceiling cloud, containing the various elements of the acoustical palette, as well as lighting, and HVAC elements. This design provides diffuse sound across the conference table, which allows a comfortable conversation level, better acoustic coverage and communication among the participants, as well as a more natural transmitted sound to the other end of the converstation. The ceiling cloud also affords a convenient approach for concealing the necessary HVAC ducts, wiring, etc. and also provides accessibility for maintenance.

\section{1:10-11:15}

Break

\section{Contributed Papers}

\section{1:15}

V6. An acoustic echo canceler implemented with AT\&T DSP16A. Sen M. Kuo and Huan Zhao (Department of Electrical Engineering, Northern Illinois University, DeKalb, IL 60115)

Acoustic echo control in teleconferencing and hand-free phone systems is not trivial because of its special characteristics. Many acoustic cancellation algorithms have been published in recent years but they are either of theoretical consideration or are computer simulations with complicated computation burdens, which makes real-time implementation impossible. In this paper, a two-band scheme is proposed to realize an acoustic echo canceler with an AT\&T WE DSP16A microprocessor. The structure of the algorithm is optimized so it can give an echo return loss enhancement (ERLE) of more than $25 \mathrm{~dB}$. A double talk detector is developed with the subband adaptive algorithm so that it will ensure full duplex communication. Performance of this canceler is evaluated with both computer simulation results and real-time test results.
11:30

V7. Adaptive acoustic echo cancellation algorithms in teleconferencing systems. Sen M. Kuo and Huan Zhao (Department of Electrical Engineering, Northern Illinois University, DeKalb, IL 60115)

In teleconferencing systems, acoustic echoes consist of acoustic coupling between louder speakers and microphones and the room reverberations. The echoes could be very annoying and even cause howling if the echo level is high enough. Among many ways of canceling acoustic echoes in teleconferencing system, the adaptive digital signal processing approach is very promising since adaptive filtering has the capability to trace the change of the echo path and cancel echoes actively. Adaptive algorithms to cancel acoustic echoes in both the time and frequency domains are presented, and the computer simulation results are compared in a proposed paper. The algorithms included are LMS, NLMS, LLMS, SDLMS, SELMS, SSLMS for FIR adaptive filtering and RLMS, SHARF, and Pole-Zero for IIR adaptive filtering. Performance of these adaptation algorithms will be discussed. Subband adaptive filtering algorithm, which has a faster convergence rate, will also be presented.

\section{Open Discussion}




\title{
Meeting of Accredited Standards Committee S2 on Mechanical Shock and Vibration
}

to be held jointly with the

\section{Technical Advisory Group (TAG) Meeting for ISO/TC 108 Mechanical Vibration and Shock}

\author{
S. I. Hayek, Chairman S2
}

Applied Research Laboratory, Pennsylvania State University, P.O. Box 30, State College, Pennsylvania 16801

\author{
D. F. Muster, Chairman, Technical Advisory Group for ISO/TC 108
}

4615 O'Meara Drive, Houston, Texas 77035

\begin{abstract}
Standards Cornmittee S2 on Mechanical Shock and Vibration. Working group chairs will present reports of their recent progress on writing and processing varjous shock and vibration standards. There will be a report on the interface of S2 activities with those of ISO/TC 108 (the Technical Advisory Group for ISO/TC 108 consists of members of $\mathbf{S} 2, \mathbf{S} 3$, and other persons not necessarily members of those committees) including plans for the next the meeting of ISO/TC 108, to be held in Milan, Italy from 26 March to 6 April 1990.
\end{abstract}

\author{
Session W. Musical Acoustics II and Psychological Acoustics IV: Timbre \\ Ira J. Hirsh, Chairman \\ Washington University and Central Institute for the Deaf, St. Louis, Missouri 63130 \\ Chairman's Introduction-9:00
}

Invited Papers

9:05

W1. The perception of timbre of steady-state complex tones. Reinier Plomp (Department of Otolaryngology, Free University Hospital, P.O. Box 7057, 1007 MB Amsterdam, The Netherlands)

The paper will present a review of work by the author in timbre perception. Steady-state complex tones are characterized by three perceptual attributes: loudness, pitch, and timbre. Loudness and pitch are mainly determined by a single physical parameter: loudness by intensity and pitch by the fundamental frequency. Timbre, on the contrary, is not a one-dimensional attribute: It is primarily determined by the shape of the sound spectrum and, therefore, multidimensional. This aspect of tone sensation was investigated by means of multidimensional scaling. For tones derived from musical instruments, excellent agreement between three-dimensional timbre representations and three-dimensional spectrum representations was found. The most important dimension is the slope of the spectrum: tones with strong low harmonics sound dull, tones with strong high harmonies sound sharp. Local peaks in the spectrum result in specific timbres as in speech vowels. It was found that the phase relation among the harmonics has only a minor effect on timbre. The importance of the latter finding in relation to the effect of reverberation on timbre will also be discussed. 
W2. Timbre and tone height. Roy D. Patterson (MRC Applied Psychology Unit, 15 Chaucer Road, Cambridge CB2 2EF, England)

A sound composed of all harmonics of $131 \mathrm{~Hz}$ in cosine phase produces the note $\mathrm{C} 3$ with a buzzy timbre. One can produce similar notes that are all C's but that sound "higher" than the original $C$, in at least three different ways. One can (a) attenuate the lower harmonics, (b) attenuate the odd harmonics, and (c) shift the phase of either the odd or the even harmonics. Traditionally, these perceptual changes would be regarded as timbre changes because tone chroma is fixed, but these manipulations also affect the tone height of the sound. Accordingly, experiments were performed to investigate the relationship between timbre and tone height. The stimuli were presented at six octaves with fundamentals ranging from $32-1024 \mathrm{~Hz}$. The listeners judged the octave of the sounds on a scale from 1 to 6 . The results show that waves with the same period can produce toneheight judgments that consistently differ by more than an octave, and that a substantial component of many presumed timbre differences (e.g., between a piano and a harpsichord) is actually a tone-height difference.

\section{0:05}

W3. Pitch and timbre interaction in isolated tones and in sequences. Paul Iverson and Carol Krumhansl (Department of Psychology, Uris Hall, Cornell University, Ithaca, NY 14853-7601 )

The interaction of pitch and timbre of tones in isolation and in sequences was studied in a series of experiments. Experiment 1 investigated whether pitch, timbre, and duration interact in the detection of changes between tone sequences. It was found that variations in timbre interfered with the detection of pitch changes, as did the variation of pitch when detecting timbre changes. Changing durations did not interfere with detection of either timbre or pitch changes. Experiment 2 investigated whether the interaction between timbre and pitch is a consequence of changes of relations between tones in the sequences. Contextual variation between sequences in pitch interfered with pitch judgments, but no other contextual interference was found. Experiment 3 measured reaction times of various classifications of isolated tones varying in pitch and timbre and found that pitch and timbre interact. These results indicate that pitch and timbre interact at the level of individual tones, they do not interact at a structural level, pitch is coded relationally, and timbre is not coded relationally. [Work supported in part by a Grant-in-Aid of Research from Sigma Xi, The Scientific Research Society.]

\section{0:35}

W4. Interaction of timbre and pitch in spectral discrimination tasks using complex tones. Punita $G$. Singh (Central Institute for the Deaf, St. Louis, MO 63110)

The dual nature of pitch ("spectral"/"virtual") and its relation to such timbral percepts as "sharpness" and "roughness" is explored in a set of experiments designed to ascertain perceptual cues facilitating discrimination tasks with complex tones having flat spectral envelopes. Three types of complexes are employed: (1) harmonic "residue" tones comprising four harmonics, (2) ten-component harmonic and inharmonic complexes with all components shifted from some reference frequency, and (3) ten-component complexes with a single component shifted from its harmonic frequency. Using two-tone sequences as stimuli, listeners are asked to judge if the second tone is (1) the same, (2) higher in pitch, (3) lower in pitch, (4) different in "something else," (5) different in "something else" and higher, or (6) different in "something else" and lower in pitch than the first ("something else" is taken to be synonymous with "timbre"). For residue tones, data indicate that changes in spectral locus yield changes in timbre. Further, direction of locus change can indicate pitch change, despite little or no change in $F 0(0 \%-2 \%$ for 200 - and $400-\mathrm{Hz} F 0)$. This implies that changes in timbral "sharpness" may be construed as pitch changes, given the absence of other cues. For stimuli in which components are shifted from harmonic frequencies, the unitary sensation of a complex may be replaced by one of multiple sources. Low components $(n=1-3)$ are more susceptible to being "heard out" as individual entities, while changes in higher components may yield changes in timbre, such as "roughness." Thus comparisons of components based on factors such as (1) magnitude, (2) location, and (3) direction of spectral change in a sequence can guide grouping operations, in addition to indicating changes in an overall property of a complex as a whole, such as its pitch, timbre, or both of these percepts simultaneously. [Work supported by AFOSR.]

\section{1:05}

W5. Timbre and the perceptual organization of musical patterns. David Wessel (Center for New Music and Audio Technologies, Department of Music, University of California, Berkeley, Berkeley, CA 94720)

Timbre, usually defined as the condition of attributes other than pitch, loudness, and duration, plays a strong role in determining the perceptual organization of musical patterns. Timbre's primary organizational influence appears to be on perceptual grouping, as in auditory stream segregation and rhythmic segmentation. Grouping by timbre can influence the tonal implications of otherwise ambiguous pitch material. Extensions of the author's previous work on the perception of timbral analogies demonstrate that timbre melodies and timbrally based phrasing and articulation can exhibit invariances similar in character to the manner in which pitch patterns exhibit perceptual invariance under transposition. With the strong organizing influence of 
timbre in mind, it is argued that care should be taken in experiments on melodic and rhythmic organization to control for the influence of timbre. Pure tones and fixed spectra exhibit a strong correlation between fundarnental frequency and measures of the location of the spectral envelope, an important timbral determinant and are inappropriate for many experiments on music perception. A simple set of procedures for preparing stimuli with independent control of spectral envelope, temporal envelope, and frequency content is provided and demonstrated.

\section{Contributed Paper}

11:35

W6. Effect of spectrum and attack properties on the evaluation of concurrently sounding timbres. Gregory Sandell (Northwestern Computer Music, Northwestern University, 2299 Sheridan Road, Evanston, IL 60208)

The perception of concurrently sounding musical timbres underlies musical orchestration, but current knowledge about psychological attributes of timbre emphasizes successive comparison (e.g., similarity, analogy). The psychological attribute "blend" was investigated by having eight listeners use a ten-point scale (a continuum of "separated" to "fused") to rate all possible simultaneous presentations of synthesized instrumental tones used in an earlier study [ J. Grey, J. Acoust. Soc. Am. 61, 1270-1277 (1975) ]. Following Grey's three-dimensional model, the data were analyzed for the effects of harmonic synchrony, spectrum content (centroid), and attack characteristics. The latter two attributes showed a significant relationship to blend: in general, "dark" instruments and instruments with quick, clean attacks tended to blend well. The amount of blend reported for such instruments decreased as their pairs increased in brightness or attack length, and pairs of bright or noisy-attack instruments did not blend well. The results found here corroborate some of the recommendations for desirable blends in traditional orchestration manuals. Other aspects likely to pertain to orchestration are interactions between sounds (e.g., masking, acoustic consonance) and the differential salience of timbres (e.g., recognizability, loudness, stream segregation); these will be evaluated.

Open Discussion 


\title{
Session X. Engineering Acoustics II and Noise III: Acoustic Metrology: Measurements, Calibration, and Standards
}

\author{
George S. K. Wong, Chairman \\ Laboratory for Basic Standards, Division of Physics, National Research Council, Ottawa, Ontario K1A OR6, Canada
}

\author{
Invited Papers
}

X1. Calibration of underwater acoustic transducers at NRL/USRD. A. L. Van Buren and J. E. Blue (Underwater Sound Reference Detachment, Naval Research Laboratory, P. O. Box 8337, Orlando, FL 328568337)

At the beginning of World War II, the United States recognized the need for establishing systematic methods for calibrating and evaluating sonar transducers and established the Underwater Sound Reference Laboratory. The Underwater Sound Reference Detachment of the Naval Research Laboratory evolved from this beginning and now serves as the focus for underwater acoustic metrology in the United States. This paper covers the measurement facilities and methods for carrying out the metrology function. Evaluation of sonar transducers and some of the ongoing research in calibration measurement methodology are also discussed. Finally, some comments are made about calibration system requirements for implementing new measurement techniques.

\section{1:25}

X2. Precision electroacoustical measurements at the NIST (formerly the NBS): Selected examples. Victor Nedzelnitsky (National Institute of Standards and Technology, Sound Building (233), Room A147, Gaithersburg, MD 20899-0001)

In addition to primary calibrations determining the pressure and free-field sensitivity levels of laboratory standard microphones by reciprocity techniques, a broad variety of electroacoustical measurements are performed at the NIST. These include the calibration and characterization of measuring microphone systems, audiometers and audiometric earphones, and other instruments at very low sound-pressure levels, as well as the investigation of the linearity of these instruments over relatively large dynamic ranges. Techniques and apparatus used for these purposes include free-field measurements in the NIST anechoic chambers and soundpressure measurements in acoustic couplers. Separation of signals from noise is achieved by means including analog filters, synchronous detectors (lock-in amplifiers), and dynamic signal analyzers. These incorporate a variety of excitation source types (e.g., sine-wave, chirp, bandlimited-impulse, periodic noise, and random noise signals) and signal-processing techniques (e.g., FFT, extraction of the fundamental component of a sinewave signal from a digitized time record). Specific examples from the NIST research and measurement services are discussed.

X3. Acoustical instrument calibration and standards. George S. K. Wong (Laboratory for Basic Standards, Division of Physics, National Research Council, Ottawa, Ontario K1A OR6, Canada)

In the field of acoustical metrology, one often encounters questions such as: Why do we need to calibrate instruments from time to time and at what intervals? How does one select an acceptable calibration procedure to implement traceability with a specific uncertainty? In order to have confidence in measurements, the overall measuring system should be calibrated in accordance with the requirements of a standard or an accepted procedure. In general, "type testing" refers to tests that are essential to verify the design integrity of the instruments; "calibration" refers to tests that are designed to monitor operational performances which may change with the passage of time; and "fieldcheck" is a relatively simple procedure, which can be performed in situ, to give some indication of whether the instrument is operational and functioning properly. To implement traceability for a specific uncertainty of a calibration procedure is a completely different matter. Examples and factors that determine the calibration schedule are discussed. 
X4. Acoustic calibration requirements in an aerospace industry environment. L. Kasturi Rangan and Klaus Jaeger (Lockheed Missiles and Space Company, Inc., 0/4875, B/195A, Sunnyvale, CA 94088-3504)

Several types of acoustic measuring instruments are used at Lockheed Missiles and Space Company's various test facilities. Lockheed's Primary Standards Laboratory is required to calibrate these instruments. In this paper, the environments where the instruments are used, calibration requirements, traceability requirements and how they are met, calibration recall intervals, and uncertainties in the calibration standards hierarchy will be presented.

\author{
Session Y. Physical Acoustics IV and Engineering Acoustics III: Localized Wave Modes \\ Bill D. Cook, Chairman \\ Department of Mechanical Engineering, University of Houston, Houston, Texas 77204-4792
}

Chairman's Introduction-1:00

Invited Papers

1:05

Y1. Localized wave transmission physics and engineering. Richard W. Ziolkowksi (Engineering Research Division, P.O. Box 5504, L-156, Lawrence Livermore National Laboratory, Livermore, CA 94550)

Exact solutions of the scalar wave equation that describe localized transmission of wave energy will be reviewed briefly. These acoustic solutions can be optimized so that they are localized near the direction of propagation and their original amplitude is recovered out to extremely large distances from their initial location. Pulses with these very desirable localized wave transmission (LWT) characteristics may have a number of potential applications in the areas of directed energy applications, secure communications, and remote sensing. The following will be shown. (1) The underlying physics of the LWT effect is closely connected to the additional degree of freedom obtained by coupling the usually disjoint portions of phase space. Position and frequency spectra at different locations in the aperture are different but are highly correlated so that the effective frequency of the aperture is higher than expected and the resulting pulses from these aperture sources reconsitute the frequency content of the LWT packet, hence its pulse shape, as it propagates. (2) The LWT solutions do not violate any known uncertainty relations and satisfy a generalized full phase-space uncertainty relation. (3) The independently addressible element LWT arrays can be designed to dramatically outperform conventional $\mathrm{cw}$-driven apertures. This includes pulse shape, peak amplitude, and energy fluence. New acoustic experimental data that corroborate these theoretical results will also be presented. [This work was performed by the Lawrence Livermore National Laboratory under the auspices of the U.S. Department of Energy under Contract No. W-7405-ENG-48.]

Y2. Nondiffracting Bessel optics: Theory and practice. J. H. Eberly (Department of Physics and Astronomy, University of Rochester, Rochester, NY 14627)

The theoretical foundation for the nondiffracting Bessel beams identified in 1985 by Durnin will be explained briefly, and several practical realizations of these beams in optical contexts will be described.

\title{
1:45
}

Y3. Experimental evidence of localized wave phenomena. D. Kent Lewis, Steven E. Benson, Floyd C. Kirk (Mechanical Engineering Department, Lawrence Livermore National Laboratory, Livermore, CA 94550), and Bill D. Cook (Cullen College of Engineering, University of Houston, Houston, TX 77204-4792)

Recent interest in creating beams that exhibit more localized behavior on the propagation axis than traditional solutions has led to many formalisms for creating and describing energy transport. One set of these 
solutions, the localized linear wave solution developed by Ziolkowski, has been under investigation through acoustic experiments for some time. Experiments using linear superposition to simulate two-dimensional acoustic arrays are discussed. These experiments use two methods, acousto-optics, to simulate an array of line sources and a point detector, as well as the traditional two-transducer arrangement, to simulate square arrays of point sources. Preliminary experiments with an actual two-dimensional acoustic array are also described. The technique of signal preprocessing to overcome damping problems and interelement interactions is discussed. [Work performed by the Lawrence Livermore National Laboratory under the auspices of the U.S. Department of Energy under Contract No. W-7405-ENG-48.]

2:05

Y4. Propagation of localized pulse trains in a circular acoustic waveguide. I. M. Besieris, A. M. Shaarawi (Bradley Department of Electrical Engineering, Virginia Polytechnic Institute and State University, Blacksburg, VA 24061), and R. W. Ziolkowski (Engineering Research Division, Lawrence Livermore National Laboratory, P.O. Box 808, L-156, Livermore, CA 94550)

A brief overview of a novel approach to the synthesis of wave signals [cf. I. M. Besieris, A. M. Shaarawi, and R. W. Ziolkowski, J. Math. Phys. 30, 1254 (1989)] will be presented. This approach, referred to as the bidirectional method, was originally introduced in order to understand the salient features of Brittingham-like solutions. Its scope is broader, however, and encompasses classes of problems altogether different from wave propagation in an unbounded homogeneous domain. The efficacy of the bidirectional method in geometrics involving boundaries has already been demonstrated [cf. A. M. Shaarawi, I. M. Besieris, and R. W. Ziolkowski, J. Appl. Phys. 65, 805 (1989) ]. In this presentation, the propagation of localized pulse trains in an infinitely long, circular, acoustic waveguide will be examined in detail. The farfields radiated out of a semiinfinite, circular, acoustic waveguide, excited by a localized initial pulse, will also be studied. These approximate solutions, which are computed using Kirchhoff's integral formula with a retarded Green's function, are causal, have finite energy, and exhibit a slow energy decay.

Y5. Pulsed focused fields. Leopold B. Felsen (Department of Electrical Engineering and Computer Science, Polytechnic University, Farmingdale, NY 11735)

Highly concentrated clumps of field energy that move through an environment without diffusing are of interest for a variety of applications. The basic problem is the synthesis of source distributions over an initial aperture to achieve this objective. Various analytical approaches have been used to explore how initial transient source field distributions can be tuned up so as to generate compact wavepackets that stay together indefinitely. The meaning of "staying together" and "indefinitely" depends strongly on how the problem is defined and has led to some startling conclusions in the early phases of investigation. At present, there seems to be agreement that no radically new phenomena are operative here but that interesting pulse shapes can be synthezied by clever spectral tuning of input conditions. Various techniques-based on direct time-domain synthesis, real and complex spectral synthesis, and use of transient beam-type basis elements-are reviewed within the context of causality and finite aperture size. Also discussed is a modeling scheme whereby a pulsed focused beam is generated analytically by assigning complex values to the source location and initiation time. This parametrization is useful for converting spherical pulse interaction with a perturbating environment directly into focused pulse interaction with that environment. [Work was supported by the Innovative Science and Technology Office through the U.S. Army Harry Diamond Laboratory.]

\section{Contributed Papers}

\section{2:45}

Y6. An experimental study of the field profile of a Bessel beam ultrasonic transducer. D. K. Hsu, F. J. Margetan, and D. O. Thompson (Ames Laboratory, Iowa State University, Ames, IA 50011)

The first experimental investigation of ultrasonic beams produced by a Bessel transducer is reported. Bessel sources are of particular interest in attempts to achieve diffractionless beams; Durnin et al. [Phys. Rev. Lett.
58, 1499 (1987) ] showed that a Bessel beam of light was diffraction-free. Using a novel technique of nonuniform poling, the polarization strength of a piezoelectric ceramic disk was made to follow the pattern of an axially symmetric, truncated Bessel function $J_{0}$ in both amplitude and phase. Field profiles and propagation behavior of the Bessel ultrasonic beam were measured experimentally in a water immersion tank. The measured profiles agreed well with calculated results using a Gauss-Hermite beam model. Effects of the number of lobes, frequency, and beam width on 
diffraction behavior were investigated. [Work supported by Basic Energy Sciences, U.S. Department of Energy.]

\section{3:00}

Y7. Development of an acoustic array for generating ADEPT pulses in air. Alan P. Poindexter, Bruce D. Baker, and Bill D. Cook (Cullen College of Engineering, University of Houston, Houston, TX 772044792)

A six-ring circular array with each ring driven by separate electronics has been developed for the purposes of exploring acoustic directed energy pulse trains in air. Each array element is a small loudspeaker with 1-in.diam cone. A different signal is to be applied to each ring simultaneously. These signals come from an MACII computer with an off-the-shelf interface board. The signal must be compensated for the loudspeaker's system reponse and interaction between array elements. [This work is supported by Lawrence Livermore National Laboratory.]
Y8. Modification of the NPE computer code to describe the propagation of axisymmetric sound beams in infinite media. Gee-Pinn James Too and Jerry H. Ginsberg (School of Mechanical Engineering, Georgia Institute of Technology, Atlanta, GA 30332)

NPE is a nonlinear progressive wave equation computer code developed by McDonald and Kuperman [Comp. Math. Appl. 11, 843-851 (1985)] to evaluate transient propagation in acoustic waveguides. It is suitable for two-dimensional phenomena, as well as for radial propagation of azimuthally symmetric waves. The present study describes the modifcations required to employ NPE for the evaluation of axially propagation axisymmetric waves, particularly those associated with sound beams radiated by a baffled piston. In addition to implementing a formulation in which the propagation is essentially parallel to the axis of a set of cylindrical coordinates, it is necessary to account for the transverse spreading of the beam into an infinite medium. Another issue is the manner in which the initial waveform input to NPE is obtained. The predictions of NPE for a linear sound beam when the input is obtained from the King integral, which is an exact solution in quadrature form, are compared to the results obtained for a simple input based on assumption of planar wave behavior in the vicinity of the transducer. The results in both cases are also compared to the analytical solution for the farfield radiation pattem.

\title{
Session Z. Structural Acoustics and Vibration III: Damping in Structural Systems
}

\author{
Martin J. Pechersky, Chairman \\ Applied Research Laboratory and Department of Engineering Science and Mechanics, Pennsylvania State University, \\ University Park, Pennsylvania 16802
}

Chariman's Introduction-1:00

Contributed Papers

1:05

Z1. Dynamic modulus measurements made with the dynamic mechanical thermal analyzer. R. J. Deigan and J. J. Dlubac (Code 1965, Ship Acoustics Department, David Taylor Research Center, Bethesda, MD 20084-5000)

Polymer Laboratories' dynamic mechanical thermal analyzer (DTMA) measures the dynamic Young's or shear modulus and loss factor of viscoelastic materials. The DMTA infers the modulus and loss factor by measuring the response of a small sample to forced vibrations in a small material sample. An optimal sample geometry is selected for a given Young's modulus range by considering effects such as thermal stress, shear deflection in short beams, and system compliance. The goal is to minimize these combined errors in the dynamic Young's modulus and loss factor measurement over the full modulus range. As a result of this analysis recommended DMTA test procedures are presented.

1:20

Z2. Dynamic mechanical propertics of neoprene rubber blends. W. M. Madigosky (Naval Surface Warfare Center, 10901 New Hampshire Avenue, Silver Spring, MD 20903)

Blends of neoprene with other elastomers can be used to provide certain properties that are not obtainable with either elastomer alone. A1- though the effect of blending on the physical properties of neoprene blends has been investigated, the effect of blending on the dynamic mechanical properties has not been investigated to the same extent. Neoprene is compatible with natural rubber, SBR, NBR, polysulfide rubber, and has limited compatibility with bulyl rubber. Seventy different blends were prepared and the physical properties measured. The dynamical mechanical properties are being measured on a Polymer Laboratories DMTA and frequency shifted using the WLF equation. This paper presents data and an analysis of the samples measured thus far.

\section{1:35}

Z3. Structural damping measurements of polymer compasites with laser Doppler vibrometry. Martin J. Pechersky and Michael T. Gerusky (Applied Research Laboratory, The Pennsylvania State University, P.O. Box 30, State College, PA 16804)

Damping loss factors of three commercially available composites up to a frequency of $12 \mathrm{kHz}$ have been performed. A resonance dwell technique was used in which both the excitation and measurement were noncontact. The excitation was accomplished electromagnetically and the driving point admittance was measured with a laser vibrometer. The 25 cm-long slender beam specimens were seismically supported at two nodes for each resonance by a combination of soft springs and silk thread. The mode shapes to determine the nodes of each beam were measured prior to 
the damping measurements. Metallic specimens were also measured to determine the limit on the accuracy of the measurement. The damping factors for the composites $\left(Q^{-1}\right)$ were found to be about two orders of magnitude larger than the metallic beam and all of the composite damping factors were found to increase monatomically with the excitation frequency over the range tested. Loss factors of the composites ranged from 1 to $4 \times 10^{-3}$ at the lower frequencies to about 3 to $16 \times 10^{-3}$ at the higher frequencies.

\section{$1: 50$}

Z4. Dynamic finite element analysis of a compressionally loaded viscoelastic cylinder. R. W. Harrison (Naval Surface Warfare Center, Silver Spring, MD 20903-5000)

A nonlinear finite element program utilizing the Mooney-Rivlin strain-energy tensor was used to obtain the static deflection of a compressionally loaded viscoelastic cylinder. The deformed coordinates and the mass and stress tensors were then passed to a complementary dynamic finite element program where the vibration isolation as a function of the applied vibrational frequency and the impedance of the top surface were calculated. The predictions of the finite element programs were verified with both Instron and accelerometer data. The calculations show the effect of compressional loading on the vibration absorption response and indicate possible geometrical modifications to enhance this absorption for application in the theory of motor mount isolation.

\section{2:05}

Z5. Prediction of the loss factor of a double pipe damping system. Meng $\mathrm{Li}$ and Robert $\mathrm{G}$. White (Institute of Sound and Vibration Research, University of Southampton, Southampton SO9 $5 \mathrm{NH}$, United Kingdom)

It is well known that increasing the damping of machinery structures will reduce noise radiation due to ringing vibration. Damping in industrial pipes can be increased by various damping techniques. This study was motivated by the need to decrease the noise radiation and vibration of pipework in power plants, particularly at elevated temperatures. Based upon the physical phenomenon of the air film damping of two parallel plates, a double pipe damping system with a very small air gap was investigated. In analysis, the Flugge shell equations of motion and the NavierStokes equation for viscous fluid were employed. First, a traveling wavetype solution was taken for shell and fluid. Then, from the interface conditions between the shell and fluid, the solution for the fluid medium was expressed in terms of shell displacements. Finally, solving the fluid equation of motion gave the fluid velocity profiles and stresses in the clearance between two cylindrical shells. From the definition of energy dissipated in the fluid, the equation of loss factor of the whole system was derived. The analytical results showed a very good qualitative agreement with the experiment. The study showed that different shell modes had a different contribution to the squeezing film damping, and the optimal design of the double pipe damping system can be deduced. The theory has been extended to studying the squeezing film damping system with various kinds of viscous fluid, such as lubrication oil, etc.

Z6. Obtaining internal and coupling loss factors by a transient test method. K. Sato and N. Lalor (Institute of Sound and Vibration Research, University of Southampton, Southampton SO9 5NH, United Kingdom )

Statistical energy analysis (SEA) is taking an increasing role in determining energy flow and predicting noise reduction of machine structures. In order to set up the SEA model it is necessary to obtain the internal and coupling loss factors of each substructure either from theory or from measurement. The conventional measurement technique, in which steadystate input power is supplied by a shaker to each subsystem in turn, is very time consuming. Moreover, it is not always possible to attach the shaker in the best position due to space limitations. This paper describes a transient method of loss factor measurement, using an instrumented hammer, which overcomes the above-mentioned difficulties. The technique uses the point and transfer inertance data for the structure to calculate input powers and subsystem energies. Results obtained by this method are compared with those obtained conventionally and good agreeement is demonstrated.

Z7. Theoretical and experimental investigation of high-temperature damping treatment using ceramic-fiber interlayer between plates. $\mathrm{L}$. C. Chow and R. J. Pinnington (Institute of Sound and Vibration Research, The University of Southampton, Southampton SO9 5NH, United Kingdom)

The damping techniques available for high-temperature applications, such as in power generating plants, are very limited. As part of the investigation of high-temperature damping work being carried out in ISVR, this paper describes the theoretical and experimental assessment of damping of plates using ceramic-fiber material as an interlayer. An experimental rig that is used to measure the ceramic-fiber material dynamic properties at elevated temperature will also be described.

\title{
WEDNESDAY AFTERNOON, 29 NOVEMBER 1989 ST. LOUIS BALLROOM H, 1:00 TO 3:00 P.M
}

\section{Session AA. Underwater Acoustics IV: Computational Acoustics II}

\author{
Henrik Schmidt, Chairman \\ Department of Ocean Engineering, Massachusetts Institute of Technology, Cambridge, Massachusetts 02139
}

\section{Contributed Papers}

$1: 00$

\begin{abstract}
AA1. An adaptive two-dimensional algorithm for solving finite-difference implementations of parabolic equations. J. S. Robertson, D. C. Arney (Department of Mathematics, U.S.M.A., West Point, NY 10996-1786), M. J. Jacobson, and W. L. Siegmann (Department of Mathematical Sciences, Rensselaer Polytechnic Institute, Troy, NY 12180-3590)
\end{abstract}

In previous work algorithms have been developed for enhancing the efficient solution of finite-difference implementations of the parabolic ap- proximation that adaptively adjust range-step size [J. Acoust. Soc. Am. 86, 252-260 (1989)] and depth-step size [Proc. Second IMACS Symp. Comput. Acoust. (to appear) ]. Here, an algorithm is presented that integrates previous work, i.e., both range- and depth-step sizes are adaptively selected by a procedure within the implicit finite-difference (IFD) implementation of the parabolic approximation. An error indicator that includes components resulting from both range and depth discretization is computed at each range step, and its value is compared to a user-specified error tolerance window. If the error indicator falls outside this window, 
the range- and depth-step sizes are adjusted so that the error is equidistributed between both components. Further adjustments are not made until the indicator leaves the tolerance window. In this sense, the algorithm generates a two-dimensional grid that is optimal and that often leads to substantial decreases in run time. Examples are presented that illustrate the accuracy and efficiency of the algorithm. [ Work supported by ONR.]

\section{1:15}

AA2. Abstract withdrawn, of various types provided that appropriate boundary conditions can be expressed. The conditions are incorporated in an implicit finite difference (IFD) scheme for the parabolic equation. The integrals appearing in the computing scheme are evaluated either analytically, if possible, or numerically. For a sea bottom considered as an elastic homogeneous halfspace, an impedance boundary condition can be evaluated relating the acoustic field in the sea at the water-bottom interface with that of the elastic bottom. The condition is inserted in the IFD scheme and the pertinent integrals are evaluated numerically by means of a fast Fourier transform algorithm. Results demonstrating the satisfactory behavior of the scheme are presented for range-independent environments. However, the method is also directly applicable to range-dependent environments.

AA5. Full-wave bottom reverberation modeling. F. Tappert and F. Ryan (Naval Ocean Systems Center, Code 541, San Diego, CA 92152)

Scattering by roughness of the penetrable water-sediment interface is regarded as volume scattering and two coupled parabolic equations for outgoing and incoming waves are derived. These equations are solved using a multiple-forward-scatter/single-back-scatter approximation plus reciprocity. In this way, a novel full-wave, fully range-dependent, bottom reverberation model has been developed for long-range sub-kilohertz frequency applications. In contrast to all known existing reverberation models, scattering strength is not input as a function of vertical angle. Instead, a statistical model of seafloor roughness is used in which the local power spectrum is input together with range-dependent geophysical properties of the sediments and basement. The output is reverberation pressure level (RPL) as a function of range, bearing, and frequency. Examples of predicted RPL in the Canary Basin operational area will be shown. [Work supported by ONT.1

AA3. Computational aspects of synthetic seismograms for layered media. P. Gerstoft,") o Vilmann (Odegaard \& Danneskiold-Samsoe ApS, Kroghsgade 1, 2100 Copenhagen, Denmatk), and S. Krenk (Aalborg University, 9000 Aalborg, Denmark)

A modified direct global matrix method for computation of synthetic seismograms in a layered viscoelastic media has been developed by combining information obtained from continuous and discrete formulations. The resulting code is stable for vertical seismic profiling as well as normal horizontal profiling and has proven efficient in the generation of full-scale seismograms. The wave field is first computed in the wave slowness domain. A proper integration back to the frequency domain is a critical part of a forward modeling code. By considering the diserete wavenumber formulation it is shown that numerical integration with equally spaced integration points corresponds to a fictitious cylindrical reflective surface with diameter inversely proportional to the sampling interval. It is demonstrated that for large ranges Filon integration is a natural extension. It is demonstrated that a trapezoidal Filon integration is more accurate than higher-order Filon schemes, which generate earlier spurious arrivals. In order to yield seismograms without spurious arrivals, the wavenumber or slowness sampling must be sufficiently dense. However, in some regions, the reflectivity function is quite regular. In these regions, linear interpolation is adequate and the number of sampling points is reduced by an adaptive scheme. Finally, in the slowness domain as opposed to the wavenumber domain, the position of the peaks in the reflectivity function is nearly independent of frequency. This enables an efficient vectorization over frequency. ${ }^{\text {a) }}$ Present address: Department of Ocean Engineering. MIT, Cambridge, MA 02139.

\section{$1: 45$}

AA4. Treatment of an elastic bottom in the parabolic approximation. John S. Papadakis, Michael I. Taroudakis (Foundation for Research and Technology-Hellas. Institute of Applied and Computational Mathematics, P.O. Box 1527, 711 10, Iraklion. Crete, Greece), and Betty Mayficld (Hood College, Frederick, MD 21701)

The use of impedance boundary conditions in the solution of the parabolic equation in underwater acoustics enables the treatment of bottoms

AA6. Manifestly reciprocal scattering amplitudes for rough interface seattering. David H. Berman and Dalcio K. Dacol (US Naval Research Laboratory, Code 5163, Washington, DC 20375-5000)

A new exact expression for scattering amplitudes for rough interface scattering is presented. This expression is explicitly reciprocal and it is shown to hold for a variety of boundary conditions: Dirichlet (pressure release), Neumann (rigid surface), impedance, fluid-fluid, and fluidsolid interfaces. This expression is shown to be a convenient starting point for deriving approximations that also respect reciprocity. Examples include a small-slope approximation, a reciprocal phase perturbation approximation, and a reciprocal smoothing approximation. [ Work supported by ONR.]

AA7. A numerical treatment of the fluid/elastic interface for the potential equations. G. H. Knightly, G.-Q. Li, D. F. St Mary (Department of Mathenatics, University of Massachusetts, Amherst, MA 01002), and D. Lee (Naval Underwater Systems Center, New London, CT 06320)

Recently Shang and Lee [J. Acoust. Soc. Am. 85, 654-660 (1989)] carried out an investigation of the fluid/elastic interface boundary. The development was done in such a manner as to provide consistency between the standard parabolic approximation in the fluid medium and the potential equations for the elastic wave equations in the elastic medium. The development is designed in such a manner as to allow the use of existing implicit finite-difference marching schemes in the liquid medium. Here, an enhancement of the interface treatment is presented, stability of the schemes is discussed, and numerical comparisons are made with a known solution having an interface. [Work supported in part by ONR.] 
sional frequency-domain propagation model based on the parabolic equation (PE) method. The sea-surface scattering model computes the timedomain scattering acoustic field using multiple realizations of sea-surface spectra to give a realistic stochastic 3-D sea-surface scattering prediction. The interfacing of the two models is achieved by obtaining the output from the sea-surface scattering model as a function of mode propagation angle and redistributing the scattered field into the proper modes in the propagation model. This new field is then marched forward by the PE model until the next significant sea-surface scattering event occurs where the interfacing method is again employed. Examples will be presented using various interfacing schemes.

\title{
WEDNESDAY AFTERNOON, 29 NOVEMBER 1989 ST. LOUIS BALLROOM D, 1:15 TO 2:35 P.M.
}

\author{
Session BB. Architectural Acoustics IV, Psychological Acoustics V, and Musical Acoustics III: \\ Distinguished Lecture
}

\author{
David Lubman, Chairman \\ Hughes Aircraft Company, P. O. Box 3310, Building 600, M. S. F241 Fullerton, California 92631 \\ Chairman's Introduction-1:15
}

\section{Invited Paper}

1:20

BB1. Binaural technology: Fundamentals and applications. Jens P. Blauert (Lehrstuhl für Allgemeine Elektrotechnik und Akustik, Ruhr Universitaet, D-4630 Bochum, West Germany)

Binaural sound systems have been used in science for quite some time, for example, to provide authentic reproduction and simulation of auditory events for sound-quality evaluation. However, it is only recently that an industry started to evolve that successfully applies binaural methods to a variety of practical tasks, and supplies the necessary equipment for doing so: the binaural-technology industry. In this lecture, the fundamentals of binaural technology will be reviewed and a few current applications discussed, such as dummy-head stereophony, binaural mixing consoles, binaural room simulation, binaural noise diagnosis, virtual auditory environment, and telepresence. Special emphasis will be put on the possibilities of incorporating binaural technology into design processes in architectural acoustics. 


\title{
Session CC. Speech Communication V: Neural Networks and Hidden Markov Models
}

\author{
Howard Nusbaum, Chairman \\ Department of Psychology, University of Chicago, 5848 South University Avenue, Chicago, Illinois 60637
}

\author{
Contributed Papers
}

\section{1:15}

CC1. Neural networks for estimating articulatory positions from speech. Bishnu S. Atal and Olivier Rioul (Acoustics Research Department, AT\&T Bell Laboratories, Murray Hill, NJ 07974)

This talk describes an application of neural networks for estimating positions of various articulators (such as the tongue, the lips, etc.) in the vocal tract from the speech signal. In general, a neural network consists of a large number of interconnected computational elements. The networks that will be discussed in this paper include an input layer of nodes connected directly or through an intermediate layer of hidden nodes to an output layer. Iterative gradient search procedures are often used for determining the unknown parameters of the neural network, but these procedures are very slow for training neural networks with a large number of hidden nodes. For estimating articulator positions, it was found that the weights in the first layer could be set to fixed randorn values during the training procedure without degrading the performance of the network. The random fixed weights in the first layer permit the use of a fast noniterative procedure for determining the unknown parameters of the second layer. Tests on a vocal tract model with ten articulatory variables show that the articulator positions can be determined accurately, using a network with 500 hidden nodes in the intermediate layer, from ten LPC parameters derived from the speech output of the model.

\section{1:27}

CC2. Acoustic feature development during unsupervised learning by a neural net. Bradley S. Seebach and Nathan Intrator (Center for Neural Science, Box 1843, Brown University, Providence, RI 02912 and Division of Applied Mathematics, Brown University, Providence, RI 02912)

A biologically plausible neural network model that employs unsupervised learning was applied to various sets of CV syllables. This network has been shown to develop recognition of input signals on the basis of distinctive signal features rather than overall signal shape [ $\mathrm{N}$. Intrator and B. Seebach, Int. Neural Network Soc. Abstr. 1, Suppl. 1, 299 (1988) ]. Syllables pronounced in isolation by male and female speakers were digitized and sampled in short (8-32 ms) overlapping time windows, then filtered into overlapping critical bandwidths [E. Zwicker, J. Acoust. Soc. Am. 33, 248 (1961) ] to produce three-dimensional energy surfaces in time and frequency. A portion of these syllabic tokens was used as a training set for the net. Those remaining were used to test generalization of network solutions both within a single speaker's utterances and across speakers. For example, when trained on a single speaker's tokens, and tested for classification of place of articulation in stop consonants, the network might correctly identify approximately $80 \%$ of similar tokens from a different speaker.

\section{1:39}

CC3. A connectionist model for classifying speech into silence, glottal source, burst friction, or mixed categories. Steven J. Sadoff (Central Institute for the Deaf, 818 South Euclid, St. Louis, MO 63110)

An algorithm for classifying speech into four classes (silence, only glottal source, only burst friction, or mixed) is being developed. This scheme primarily differs from the standard silence/aperiodic/periodic classification in that the defining characteristics of glottal source and burst friction sounds do not depend on periodicity distinctions, but on the locus of the energy concentrations. One male speaker reciting the Rainbow passage has been recorded and analyzed. Utilizing a strictly layered backpropagation network, the automated learning procedure is trained using the first $30 \mathrm{~s}$ of the passage: the final $75 \mathrm{~s}$ are used for testing. Quantitative results, along with several examples illustrating this model, will be presented. Additionally, the performance and classification strategy of the network will be compared to that of human observers. [Work supported by AFOSR.]

\section{1:51}

CC4. Context-modulated discrimination of similar vowels using secondorder connectionist networks. Raymond Watrous (Department of Computer Science, University of Toronto, Toronto, Ontario M5S 1A4, Canada)

Discrimination of medial adjacent vowels in the context of voiced and unvoiced stop consonants using connectionist networks is investigated. Separate discrimination networks were generated for one speaker from samples of the vowel centers of $\{e, x]$ for the six contexts $[b, d, g, p, t, k]$. A single context-independent network was similarly generated. The context-specific error rate was $1 \%$, whereas the context-independent error was $9 \%$. A method for merging isomorphic networks into a single network is described. The method uses singular value decomposition to find a minimal basis for the set of context-specific weight vectors. Context-dependent linear combinations of the basis vectors may then be computed using second-order network units. Compact networks can thus be obtained in which the vowel discrimination surfaces are modulated by the phonetic context. In a preliminary experiment, as the number of basis vectors was reduced from 6 to 3 , the error rate increased from $1 \%$ to $3 \%$. Experiments with nonlinear optimization of context-modulated, secondorder networks for this task are underway and will be reported.

\section{2:03}

CC5. Automated language acquisition. A. L. Gorin and S. E. Levinson (Speech Research Department, AT\&T Bell Laboratories, Murray Hill, NJ 07974)

A new approach to developing large-vocabulary speech understanding systems, in which the system automatically acquires a language model for its task based on semantic information, is introduced. This is in contrast to previous work, in which language was preprogrammed. An important consequence of this approach is that it leads to habitable language models. To accomplish this task, use is made of a medium-grain neural network, together with a novel adaptive training procedure for estimating the set of connection weights. The resulting connection weights have an information-theoretic interpretation, and do not require gradient search techniques for their estimation. A conversational-mode system that serves as a test bed for the network is described. The application scenario is inward-call management, where a customer telephones a large organization and encounters an operator whose function is to forward the call to 
one of several departments. The network's performance is first experimentally evaluated on three text databases: sentences collected from the inward-call management system, transcriptions of customer responses to an automated call transaction system (ACTR), and a collection of idiomatic phrases connoting yes and no. Finally, preliminary results evaluating the inward-call management system using speech data comprising unconstrained sequences of isolated words, which are phonetically transcribed via an ergodic HMM and provided as noisy input to the network, are reported.

\section{2:15}

CC6. HMM-based word recognition using word category prediction neural network. Katsuteru Maruyama, Masami Nakamura, Takeshi Kawabata, and Kiyohiro Shikano (ATR Interpreting Telephony Research Laboratories, Sanpeidani Inuidani Seika-chou, Souraku-gun, Kyoto 619-02, Japan)

Word category prediction is used to implement an accurate word recognition system. Traditional statistical approaches require considerable training data to estimate the probabilities of word sequences and many parameters to memorize probabilities. It is also difficult to predict unseen data. To solve this problem, NETgram (Neural Network for word category prediction) has been proposed. The performance of the NETgram was comparable to that of the statistical trigram model [M. Nakamura et al., Proc. IEEE ICASSP '89, p. 731, s13.10 (1989.5) ]. In order to evaluate the ability of the NETgram, the NETgram is applied to an English word recognition experiment based on HMM. English phone models for HMM were trained using the forward-back ward algorithm according to the pronunciation symbols of training speech. The NET gram is efficiently applied to improve word recognition results using a dynamic programming technique. The word recognition rate using HMM is $81.0 \%$. The NETgram (trigram model) trained by 512 sentences in the Brown Corpus Text Database improves the word recognition rate to $86.3 \%$. The statistical trigram model improves the word recognition rate to $85.4 \%$. The NETgram performs better than the statistical trigram model although the training data are insufficient to estimate the probabilities of the trigram word category sequence. This experiment shows the NETgram can learn general characteristics from limited training data, that is to say, the NETgram can interpolate sparse training data to a certain degree.

CC7. Minimum mean-square error speech enhancement using hidden Markov models. Yariv Ephraim (Speech Research Department, AT\&T Bell Laboratories, 600 Mountain Avenue, Room 2C-572, Murray Hill, NJ 07974)

A minimum mean-square error (MMSE) estimation approach for enhancing speech signals degraded by statistically independent additive noise is developed. This approach is motivated by the fact that optimal waveform coding in the MSE sense, and optimal autoregressive model vector quantization in the Itakura-Saito sense, given noisy speech, can be performed using MMSE estimators of the speech waveform and its sample spectrum, respectively. Estimators for the clean speech signal, and for the spectral magnitude and sample spectrum of that signal, are developed, based upon hidden Markov modeling of the clean signal and the noise process. The parameters of the models for the two processes are estimated from training sequences of clean speech and noise samples. It is shown that the MMSE estimator of the clean signal comprises a weighted sum of
Weiner filters, one for each state of the hidden Markov model for the clean speech, where the weighting factors at each time instance are the probabilities of the filters to be the correct filters for the given noisy speech. The other MMSE estimators considered here have similar structure. At 10-dB input signal-to-noise ratio (SNR), the proposed approach provided an improvement of 4.5-5.5 dB in SNR.

\section{2:39}

CC8. Speech recognition using HMMs with an LVQ-trained codebook. Hitoshi Iwamida, Shigeru Katagiri, and Erik McDermott (ATR, Sanpeidani Inuidani, Seika-cho Soraku-gun, Kyoto 619-02, Japan)

A new speech recognition algorithm using HMMs with a codebook trained by learning vector quantization (LVQ) is here described. Both HMMs and LVQ are stochastic algorithms holding considerable promise for speech recognition. In particular, HMMs have the significant advantage that phone models can easily be concatenated to produce a long utterance model, such as a word or sentence model. LVQ, on the other hand, is a powerful classifier, as shown in the high phoneme recognition rates obtained in McDermott and Katagiri (1989). The new algorithm described here combines the advantages inherent in each of these two algorithms. To evaluate this LVQ-HMM hybrid, phoneme recognition experiments were performed using the same data as used in TDNN (Waibel, 1988) and shift-tolerant LVQ (McDermott and Katagiri, 1989) experiments. Applied to various phoneme recognition tasks, the LVQHMM hybrid achieved recognition rates much higher than those of a conventional HMM with a codebook designed by $K$-means clustering. For small codebook sizes, the hybrid model realized a more than tenfold decrease in recognition error rates, yielding extremely high phoneme recognition performance, comparable to that of TDNN or shift-tolerant LVQ

CC9. A new discriminative HMM training procedure. Eng Fong Huanga) and Frank K. Soong (Speech Research Department, AT\&T Bell Laboratories, 600 Mountain Avenue, Murray Hill, NJ 07974)

A training procedure is proposed for improving the discriminative power of a maximum likelihood (ML) hidden Markov model (HMM) without sacrificing its classification capability. The proposed discriminative HMM consists of a conventionally trained ML model and a discriminative model. The training data are utilized in two different modes. In the first mode, conventional ML models, denoted as master models, are trained. In the second mode, discriminative models, denoted as slave models, are trained by aligning training tokens of a certain word with all but the correct word master models and the model parameters are estimated by maximizing the conditional likelihood of the training tokens given the fact that they are aligned with incorrect-word master models. In recognition, the scores of the master models are reinforced by the scores of the input tokens, which are compared with corresponding slave models. A speaker-independent, 39-word, alpha-digit database was used to evaluate the new training procedure. Experimental results indicate that the new training can improve the recognition performance by $1 \%-2 \%$. However the discriminative power of the slave models decreases gradually when more sophisticated models and features are used. " On leave from Telecommunication Laboratories, Chung-li, Taiwan. 


\section{Plenary Session}

Harvey H. Hubbard, Chairman

President, Acoustical Society of America

\section{Business Meeting}

Presentation of Awards

Distinguished Service Citation to Daniel W. Martin Silver Medal in Bioresponse to Vibration to Floyd Dunn Silver Medal in Engineering Acoustics to Joshua E. Greenspon

Silver Medal in Musical Acoustics to Max V. Mathews

\section{Entertainment}

“Bingham's Missouri” will be presented by the St. Louis Chapter of Young Audiences, Inc. This national award-winning presentation, featuring music from the Missouri Folk Trio, chronicles sights and sounds along the Missouri River during the 1800 s, as viewed by celebrated American artist George Caleb Bingham. 


\title{
Session DD. Animal Bioacoustics I: Animal Bioacoustics and Noise
}

\author{
William C. Cummings, Chairman \\ Oceanographic Consultants, 5948 Eton Court, San Diego, California 92122-3203
}

\author{
Invited Papers
}

8:00

DD1. Animal psychoacoustics. Richard R. Fay (Parmly Hearing Institute, Loyola University of Chicago, Chicago, IL 60626)

The sense of hearing in animals has been studied quantitatively using a variety of behavioral techniques for the past 50 years. The database now existing [R. Fay, Hearing in Vertebrates: A Psychophysics Databook (HillFay Associates, Winnetka, IL, 1988) ] includes data for 180 vertebrate species and allows several generalizations to be made regarding the relations between human and animal hearing, the methodologies useful in animal hearing studies, and the hearing processes common to all vertebrates. Although the frequency range of hearing varies by many octaves among vertebrates, absolute sensitivity at best frequency is quite similar ( \pm 10 $\mathrm{dB}$ re: $0.0002 \mathrm{dyn}^{-2}$ ). The sound discrimination performance often, but not always, exceeds the performance of animal listeners. There are no clear phylogenetic trends toward more acute discrimination abilities in primates, and very few examples of specializations for enhanced sound discrimination abilities among vertebrates (echolocators may be exceptions). There is no single conditioning or psychophysical method that clearly gives lower or more valid thresholds. Data from the literature will be presented to illustrate these and other general conclusions about animal hearing.

\section{8:25}

DD2. Echolocation signals of odontocetes in open waters. Whitlow W. L. Au (Naval Ocean Systems Center, Kailua, HI 96734)

The characteristics of echolocation signals used by the Atlantic bottlenose dolphin (Tursiops truncatus) and by the beluga or white whale (Delphinapterus leucas) in target detection and discrimination experiments in open waters will be discussed. Echolocation signals measured in open waters have been found to have considerably different properties than those measured in tanks. The primary reason for the differences in echolocation signals may be attributed to the use of very-low-intensity signals by animals in tanks and the use of higherintensity $(>40 \mathrm{~dB})$ signals in open waters. Signals are projected in the forward direction along a narrow beam and echoes are detected by a receiving beam that overlaps the transmission beam. Acoustic properties of these signals, such as source sound-pressure levels, signal waveform, frequency spectrum, peak frequency, bandwidth, and click intervals, will be considered. The acoustic characteristics of echolocation signals propagating from the animal's head will also be discussed. The structure of the acoustic field directly forward of the animal, the transition region between the near and far acoustic fields, the directivity patterns in the vertical and horizontal planes, and off-axis distortion of signals will be addressed.

DD3. Experimental verification of a spectrogram correlation theory for FM echolocation. James A. Simmons (Department of Psychology and Section of Neurobiology, Division of Biology and Medicine, Brown University, Providence, RI 02912)

The FM bat, Eptesicus fuscus, perceives each reflecting glint in a target as the cross-correlation function of its echoes, using a mixture of time- and frequency-domain auditory representations according to the time separation of echo components relative to the receiver's integration time of $350 \mu \mathrm{s}$. The accuracy of jittereddelay discrimination is as good as $10 \mathrm{~ns}$. Separate jitter discrimination curves for $0^{\circ}$ and $180^{\circ}$ phase shifts of echoes fit together in the manner of compound period histograms to form the complete cross-correlation function of echoes. Jitter discrimination curves shift along the delay axis by $-17 \mu \mathrm{s} / \mathrm{dB}$ when echo amplitude changes (amplitude-latency trading), showing that the bat uses timing of neural discharges rather than extraneous, spectrally encoded cues to discriminate jittered echoes. Other recent jitter experiments missed part of these results due to an unintended masking effect. A spectrogram-with-phase representation of echoes followed by a transformation of the frequency-domain component of the spectrogram into the time domain would account for the bat's perception of the cross-correlation function. This transformation is the basis for fusing target range and target shape into unified images. These results additionally implicate vertical and horizontal localization as part of a single imaging process. 
9:15

DD4. Sonar signal design of the Chinese bat Pipistrellus abramus temmink and the French bat Myotis mystacinus during natural hunting prey. Zhenbiao Lin, Jin Lu (Department of Radio Engineering, South China University of Technology, Guangzhou, People's Republic of China), and Bernard Escudie (Laboratory of Signal Processing, ICPI, 25 rue du Plat, Lyon, France)

There are more than 100 species of bats in China. This paper gives the first description of Chinese FM bat signals and a comparison with the French FM bat. Twenty signals emitted by a South China bat, Pipistrellus abramus temmink (P.a.t.), and $\mathbf{4 9}$ signals by a French bat, Myotis mystacinus (M.m.), during searching, identification, pursuit, and prey capture in the field were studied via time, frequency analysis, and 3-D wideband ambiguity diagrams. The evolution of main signal parameters (duration, dominant frequency, bandwidth, and average sampling pace along the hunting track) was described as a function of estimated bat-prey distance in order to find the relation between the parameters and distance. The results suggest that the P.a.t. bat and the M.m. bat use a similar strategy of signal design although they are living in different continents, and hunting different insects in different environments. For two species of FM bats, a turning point in the parameter evolution exists at about $1.5 \mathrm{~m}$ between bat and prey. In our recorded signals, the M.m. bat emits much larger bandwidth and stronger harmonics than the P.a.t. bat. As a consequence, the range resolution and bearing resolution are higher for the M.m. bat than for the P.a.t. bat in this experiment. [Work supported by NSFC 1881155 of China and RCP 445 CNRS of France; partially supported by NIH grant NS 20527 to Professor P. Jen of the University of Missouri-Columbia, Columbia, MO.]

\section{9:30}

DD5. Detection of intensity decrements by the chinchilla. Christine J. Graf, Samuel S. Saunders, and Richard J. Salvi (Hearing Research Laboratory, Department of Communicative Disorders and Sciences, State University of New York at Buffalo, Buffalo, NY 14214)

Three monaural chinchillas were trained to detect intensity decrements in broadband noise $(20 \mathrm{kHz})$ using a shock-avoidance conditioning procedure. The intensity decrements were presented at one of nine different durations between 2 and $35 \mathrm{~ms}$ at noise levels of 25,45 , and $65 \mathrm{~dB}$
SPL. At each intensity-duration combination, the level of the decrement was varied to obtain a decrement threshold. The minimal detectable decrement decreased from approximately $20 \mathrm{~dB}$ at the shortest duration to an asymptote of roughly $4 \mathrm{~dB}$ at approximately $30 \mathrm{~ms}$. The data were modeled by a low-pass filter with an 11 -ms time constant. The decrement detection function of the chinchilla is similar to that of humans. However, long duration decrement thresholds are larger in the chinchilla, as would be predicted from the large intensity difference limen of the chinchilla. In general, there was little change in the decrement function across background intensities except that 2-ms decrements were not detected at the 25 dB SPL background intensity. [Research supported by grants RO1NS16761 and RO1NS23894.

\section{9:45}

DD6. An old cochlear frequency-position function compared to more recent data. D. D. Greenwood (Audiology/Speech Sciences, University of British Columbia, Vancouver, British Columbia V6T IW5, Canada)

Accurate cochlear frequency-position functions based on physiological data would assist interpretation of physiological and psychoacoustic data within and across species. In 1961, a family of almost-exponential functions was developed [D. D. Greenwood, J. Acoust. Soc. Am. 33, 1344-1356 (1961)] by integrating an exponential function fitted to a subset of critical bandwidth estimates. The integral fitted well cochlear data on frequency-place in human cadavers, and, with changes in constants, similar data on several other species-as well as behavioral-anatomical data on cats. Since 1961, mechanical data from dead human ears and various data from living animals (cat, guinea pig, chinchilla, monkey, gerbil) have appeared. It is shown that they are fitted by the same basic function. As in 1961, in both cadaver and living ears, the same slope parameter can be used if partition length is normalized to 1 ; i.e., slope is scaled if distance is expressed in physical units. Slope uniformity suggests that the cadaver data may well describe at least the function's slope in living human cochleas. To set the upper frequency limit requires only the empirical choice of a single other parameter. It is suggested that these cochlear frequency-position functions be used in plotting not only physiological data but also psychoacoustic data (and in modeling work with speech and other bioacoustic signals), since, if the cochlear data are correct, the functions are consequently independent of, and an appropriate means to examine, psychoacoustic data and assumptions. [Work supported by NSERC, Canada.]

\section{0:15}

DD7. Measurement of the low strain complex extensional modulus of rabbit skin. J. Michael Pereira, Joseph M. Mansour, and Bryan R. Davis (Department of Mechanical and Aerospace Engineering. Case Western Reserve University, Cleveland, $\mathrm{OH}$ 44106)

The complex extensional modulus of rabbit skin was measured by recording acoustic pulses propagating along thin strips of skin excised from the backs of rabbits. In the experiment, a step change in displacement was introduced into the skin sample by a speaker, to which one end of the sample was attached. The response was recorded at different locations along the sample by a phonograph pickup. The nominal static stress on the samples during the experiments was maintained at a low level ( $1500 \mathrm{~Pa}$ ). Repeatable results for the attenuation, speed, and viscoelastic storage and loss moduli were measured in the frequency range of $200-$ $1000 \mathrm{~Hz}$. In this Irequency range, the attenuation was approximately lin- early proportional to the frequency and the speed was approximately constant. The storage and loss moduli were also approximately constant in this frequency range, with mean values of $179300 \mathrm{~Pa}$ and $113700 \mathrm{~Pa}$, respectively. [Work supported by NIH.]

\section{0:30}

DD8. Improved spatial resolution of fish using hydroacoustics. $P$. H. Patrick, B. Sim, and G. Hunt (Biological Research Section, Ontario Hydro Research Division, 800 Kipling Avenue, Toronto, Ontario M8Z 5S4, Canada)

Investigations were conducted in the laboratory on the accuracy of a commercial sonar system in detecting underwater targets. Accurate spa- 
tial resolution of fish is necessary for reliable estimates of biomass and for classifying fish species. Results suggested that the sounder was limited in target separation below $25 \mathrm{~cm}$ at a transmitted pulse of $0.1 \mathrm{~ms}$. Modifications to equipment resulted in an improved resolution (to approximately $10 \mathrm{~cm}$ ) of targets in both vertical and horizontal tank experiments. Target strength estimates were also determined. The application of hydroacoustics to detect and classify fish is discussed. [Work supported by Ontario Hydro.]

\section{0:45}

DD9. Ineffectiveness of ultrasonic flea collars. Michael W. Dryden, Glenis R. Long, and Sayed M. Gaafar (Departments of Veterinary Pathology and Audiology and Speech Science, Purdue University, West Lafayette, IN 47907)

Ultrasonic flea collars from two companies were evaluated for their ability to reduce flea numbers on cats with experimentally induced flea infestations. Sounds produced by the collars were also evaluated both before and after use to ensure that the flea collars were functional. Each brand of ultrasonic flea collar was evaluated on five cats with each cat being infested with 50 cat fleas (Ctenocephalides felis) for a 7-day treatment and control period. Cats were declawed and fitted with Elizabethan collars to reduce normal grooming activity. Collars generated $40-\mathrm{kHz}$ pulses (0.8-ms exponential rise and fall) every 50 or $20 \mathrm{~ms}$ (depending on the device). The level of the stimuli was between 92 and $80 \mathrm{~dB} \mathrm{SPL} \mathrm{at} 10$ $\mathrm{cm}$ and would thus be audible to both cats and dogs. An average of $99.6 \%$ and $97.4 \%$ of the fleas were still on the cats after treatment and control periods, respectively. The ultrasonic flea collars were totally ineffective at reducing flea numbers on these cats.

\section{1:00}

DD10. Kurtosis measurements: Implications for noise exposure criteria. R. P. Hamernik, K. D. Hsueh, W. A. Ahroon, R. I. Davis, and G. A. Turrentine (Auditory Research Laboratory, 107 Beaumont, State University of New York, Plattsburgh, NY 12901)

Two conventional measures of an industrial noise environment are the overall sound-pressure level (SPL) and the power spectrum. While these two measures are necessary, they may not be sufficient to describe a noise environment for the purposes of evaluating the hazard to hearing. This presentation will describe the results of an experiment designed to test the hypothesis that for equal SPL and power spectrum a high-kurtosis noise exposure is more hazardous to hearing than is a low-kurtosis noise. Two groups of chinchillas, with two animals per group, were exposed continuously for 5 days, to one of two classes of noise at a $90 \mathrm{~dB}$ SPL. The power spectrum of both noises was identical but the noises had different values of kurtosis. The results clearly showed that there was up to a $20 \mathrm{~dB}$ greater permanent hearing loss for the animals exposed to the high-kurtosis noise. Detailed results of asymptotic and permanent threshold shifts and sensory cell losses will be presented. These results would appear to be a clear indication that, even for moderate levels of exposure, energy is not a suffi- cient variable upon which to base noise exposure standards. [Work supported by NIOSH.]

\section{1:15}

DD11. Effects of sodium salicylate and intense noise on thresholds in the chinchilla. B. R. Bancroft, F. A. Boettcher, and R. J. Salvi (Hearing Research Laboratory, SUNY - Buffalo, Buffalo, NY 14214)

Salicylate administration exacerbates the temporary threshold shift (TTS) caused by noise exposure in humans [D. McFadden and H. S. Plattsmier, Hear. Res. 9, 295-316 (1983) ]. However, results from animal experiments are conflicting as to whether salicylate administration potentiates noise-induced TTS. Because of the controversy in the literature and the lack of data on the potential permanent interaction of noise and salicylates, chinchillas were exposed to noise ( $105 \mathrm{~dB} \mathrm{SPL}, 0.5-\mathrm{kHz}$ octave band, 15 days), salicylate ( $300 \mathrm{mg} / \mathrm{kg}$, daily i.p. injection ), or both, concurrently. Salicylate administration resulted in a TTS of 4 to $8 \mathrm{~dB}$, depending on frequency, and no permanent threshold shift (PTS). Noise exposure caused 46 to $68 \mathrm{~dB}$ of TTS and 10 to $26 \mathrm{~dB}$ of PTS. The group exposed to both agents received 57 to $70 \mathrm{~dB}$ of TTS and 8 to $30 \mathrm{~dB}$ of PTS. There was little difference between the interaction and noise-alone groups for both TTS and PTS measures. In conclusion, salicylate did not exacerbate the temporary or permanent threshold shifts caused by intense noise exposure. [Work supported by NIH 5RO1-NS23894-03.]

\section{1:30}

DD12. The threshold recovery pattern between 48 h to 30 days after exposure to 50- $\mu \mathrm{s}$ arc-discharge impulse noise in guinea pigs. Wenyuan Gao and Fanming Yuan (Department of Navy Medicine, The Second Military Medical College, Shanghai, People's Republic of China)

The threshold recovery pattern $48 \mathrm{~h}$ after exposure to arc-discharge impulse noise was investigated. Guinea pigs, implanted with chronic electrodes, were measured in quiet using a click average-evoked response technique. The animals were exposed to ten impulses $(1 / \mathrm{min})$ of either $170-(N=10), 166-(N=17), 163-(N=15), 160-(N=9)$, or 157 $(N=16) \mathrm{dB}$ peak SPL. The A duration was $50 \mu \mathrm{s}$, and the rise time was $10 \mu \mathrm{s}$. Recovery threshold was monitored, at $48 \mathrm{~h}$, at $8,15,22$, and 30 days, respectively, after exposure. Some of the animals were then sacrificed for cochlea histology. The results were as follows. (1) The recovery patterns varied with peak amplitude of impulse noise, i.e., they were linear in $\log$ time of 157 and $160 \mathrm{~dB}$; they were nonmonotonic in log time of 163 and $166 \mathrm{~dB}$, and there was no recovery but a growth pattern at $170 \mathrm{~dB}$. (2) The period between 8 to 15 days post exposure is an important period for turning to permanent threshold shift (PTS); i.e., if the recovery goes on in this period, it will show no PTS. (3) This kind of impulse noise with short duration and rapid rise time may be more harmful to the audition than the other kind. 


\title{
Session EE. Physical Acoustics V: Mechanical Wave Propagation in Condensed Matter Physics
}

\author{
Philip L. Marston, Cochairman \\ Department of Physics, Washington State University, Pullman, Washington 99164-2814
}

Moises Levy, Cochairman

Physics Department, University of Wisconsin, Milwaukee, Wisconsin 53201

Chairman's Introduction-8:00

Invited Papers

8:05

EE1. Ultrasonic absorption in the magnetic superconducting system $\mathrm{Er}_{\mathbf{1}_{-}} \mathrm{Ho}_{\uparrow} \mathrm{Rh}_{4} \mathrm{~B}_{4}$. Keun J. Sun (NASALangley Research Center, Mail Stop 231, Hampton, VA 23665)

Ultrasonic attenuation measurements as a function of temperature at constant magnetic fields and as a function of magnetic field at constant temperatures in $\mathrm{Er}_{1-x} \mathrm{Ho}_{x} \mathrm{Rh}_{4} \mathrm{~B}_{4}$ show interesting behavior at low temperatures. The enhanced attenuation observed in the superconducting state and the sharp change in attenuation at magnetic phase transition for the superconductors in this system are attributed to spin-phonon interaction. The peculiar dependence of attenuation on orientation of magnetic field with respect to sound wave propagation direction evidences indirectly the coexistence of magnetic order and superconductivity. A twolevel model of phonon-phonon interaction is proposed to elucidate the occurrence of relaxation type attenuation maxima observed in the temperature-dependent attenuation curves of all the samples. Furthermore, the attenuation behavior at low temperatures in high magnetic fields may also manifest the occurrence of a new magnetoelastic coupling effect.

\section{8:35}

EE2. Ultrasonic attenuation measurements on the heavy fermion superconductor $\mathrm{UPt}_{\mathbf{3}}$. B. K. Sarma, M. Levy, A. Schenstrom, Y-J. Qian, M.-F. Xu (Department of Physics, University of WisconsinMilwaukee, Milwaukee, WI 53201), S. Adenwalla, J. B. Ketterson, Z. Zhao (Department of Physics, Northwestern University, Evanston, IL 60208), and D. Hinks (MST, Argonne National Laboratory, Argonne, IL 60439)

The superconducting pairing mechanism in the heavy fermion superconductor $\mathrm{UPt}_{3}$ is unconventional, as evidenced from any of its low-temperature properties. Recent ultrasonic absorption, heat capacity, and magnetization measurements show a multiplicity of phases in the $H-T$ plane, suggesting an exotic order parameter. The experimental results will be reviewed. [Work supported by ONR and NSF.]

EE3. Ultrasonic studies of collective modes in superfluid ${ }^{3} \mathrm{He}$. John B. Ketterson (Department of Physics and Astronomy, Northwestern University, Evanston, IL 60201)

Liquid ${ }^{3} \mathrm{He}$ undergoes a transition at a temperature of $2 \times 10^{-3} \mathrm{~K}$ into a superfluid state. The state is related to the paired-electron state of the BCS theory of superconductivity but differs in that the ${ }^{3} \mathrm{He}$ atoms are paired in an $L=1$ ( $\rho$ wave), $S=1$ (triplet) state [ rather than the $L=0$ ( $s$ wave), $S=0$ (singlet) state of conventional superconductivity]. Various excited states of the superfluid exist, which are referred to as excitonic or collective modes, and, loosely, they correspond to various values of the total angular momentum $J=L+S$. Some of these modes couple strongly with ultrasound. A brief review is presented of these collective modes and of the ultrasonic experiments performed to date, which probe their behavior as a function of temperature and magnetic field. 
EE4. Wave localization in anisotropic random media. Ping Sheng (Exxon Research and Engineering Co., Route 22 East, Clinton Township, Annandale, NJ 08801)

As a phenomenon generic to waves in random media, localization has many general features common to both quantum particles, e.g., electrons, and classical waves, e.g., electromagnetic and elastic waves. One such aspect is that all waves localize in two- or one-dimensional systems with an arbitrary amount of randomness, and that in three dimensions a wave localizes only in certain energy regimes that are separated from the delocalized regimes by so-called mobility edges. In this paper, the results of recent work on the anisotropic dimensional crossover behavior for wave localization are described. Starting from a randomly layered medium, some scattering centers were introduced, i.e., inhomogeneities, into each of the layers in a controlled manner. These inhomogeneities cause the wave to scatter out of the layering direction. When the strength and the density of the scattering inhomogeneities become equal to the randomness encountered in propagating from layer to layer, then the system becomes an isotropic, 3-D random medium. Therefore, in increasing the scattering strength and density of the scatterers there is essentially a "cross over" from one-dimensional randomness, where all waves are localized, to isotropic three-dimensional randomness, where there can be mobility edges. What is found is that there is a critical anisotropy below which the system behaves as $1 \mathrm{D}$ and above which the system behaves as $3 \mathrm{D}$. In other words, the transition is achieved in a discontinuous manner. The talk will emphasize the underlying physics of the localization and its anisotropic critical transition.

10:05

EE5. Surface acoustic waves on nonlinear substrates. Alexei A. Maradudin (Department of Physics, University of California, Irvine, CA 92717)

The nonlinearity of the substrate on which a surface acoustic wave propagates causes acoustical rectification of the surface wave, harmonic generation and nonlinear mixing of surface waves, and the acoustoelastic effect. It gives rise to weakly nonlinear surface acoustic waves, and to associated surface acoustic solitary waves. The spatial dispersion required for the existence of the latter is introduced by coating the substrate by a thin film with different material properties. Explicit conditions for the formation of envelope solitons are presented, as well as for self-focusing of the surface acoustic waves. The nonlinearity can also cause Love waves to become leaky with an amplitude-dependent damping constant. Finally, a periodic corrugation of the surface of a nonlinear elastic substrate is used to introduce spatial dispersion into the system. An amplitude dependence of the stop bands for the propagation of weakly nonlinear surface acoustic waves across the resulting periodic structure, and an amplitude-dependent attenuation in the case of sagittal polarization, are found. [Work supported by NSF Grant No. DMR 88-15866.]

\section{0:35}

EE6. Relating macroscopic deformation to site symmetry changes around the $\mathrm{Cr}^{+++}$in shocked ruby crystals. Y. M. Gupta (Department of Physics, Washington State University, Pullman, WA 99164-2814)

An important but very difficult problem in shock wave research is to understand and interpret timeresolved, continuum measurements in condensed materials in terms of changes at the atomic/molecular level. As a start toward this challenging problem, experimental and theoretical studies on understanding the luminescence $R$-line spectra in shocked ruby crystals have been carried out. Time-resolved experiments have been performed to obtain wavelength shifts under macroscopically, well-defined compression and tension loading, and for shock wave propagation along the crystal $c$ and $a$ axes. Below the Hugoniot elastic limit ( $\approx 13 \mathrm{GPa}$ ), the observed $R$-line spectra are strongly anisotropic and show nonlinear shifts with density for both compression and tension. For the theoretical analysis, the deformation potential is written in terms of symmetry adapted basis according to the irreducible representation of local octahedral group. Using the symmetry of the macroscopic strain, the perturbative effects of the deformation potential are evaluated for the ${ }^{2} E$ state that gives rise to the $R$ lines. Results not only from the shock experiments but also from hydrostatic and uniaxial stress experiments can be analyzed consistently using these analytic developments. A summary of the experimental and analytical work will be presented. [This work was carried out in collaboration with S. M. Sharma, X. A. Shen, P. D. Horn, and J. A. Burt and supported by ONR Contract N00014-86-0307.]

\section{1:05}

EE7. Ballistic phonon mediated detection of elementary particles in silicon. B. Neuhauser (Department of Physics and Astronomy, San Francisco State University, San Francisco, CA 94132), B. Cabrera, A. T. Lee, B. A. Young (Department of Physics, Stanford University, Stanford, CA 94305), C. J. Martoff (Department of Physics, Temple University, Philadelphia, PA 19122), and J. P. McVittie (Center for Integrated Systems, Stanford University, Stanford, CA 94305)

When an incident particle collides with an electron or nucleus in a silicon crystal, $70 \%$ of the recoil energy is converted into a burst of low-energy phonons. These phonons propagate ballistically for distances of several 
centimeters if the crystal has very few defects or impurities and if it is cooled to a temperature well below $1 \mathrm{~K}$. Direct sensing of the phonons is the basis for a new type of elementary particle detector, called a silicon crystal acoustic detector (SiCAD), which will be used in neutrino experiments and in the search for other weakly interacting particles. The first generation of ultrasensitive phonon detectors consists of a thin film strip of a superconducting material, which is current biased just below the critical temperature. Incident phonons drive a segment of the film normal and thereby produce a voltage pulse. These devices have sufficient sensitivity and resolution to detect energy depositions of several $\mathrm{keV}$ in standard 3-in. silicon wafers. Research currently is in progress on the next generation of phonon detectors that will make use of superconducting tunnel junctions. [Work supported by DOE, NSF, and Research Corporation.]

\title{
Contributed Paper
}

11:35

EE8. Using a SQUID acoustomagnetic spectrometer to characterize continuous wave acoustic response observations. Ronald K. Sundfors and Mark R. Holland (Department of Physics, Washington University, St. Louis, MO 63130)

A SQUID acoustomagnetic spectrometer is used to detect changes of magnetization of tantalum metal produced by a continuous wave composite resonator in an external magnetic field. Acoustic power variations produce temperature variations in the spectrum and thus cause changes in the Curie susceptibility of the paramagnetic impurities present in the sam- ple. Swept frequency acoustic spectra of single-crystal tantalum standing wave resonances at the normal mode frequencies (mechanical resonances) are studied at $4.2 \mathrm{~K}$. Depending on the rate of frequency sweep through a mechanical resonance, the applied acoustic power, and the magnetic field, the observed structure at the mechanical resonance frequencies can be both above and below the baseline. The low-frequency side of each structure has a Lorentz line shape and the high-frequency side has an exponential decay that is explained by heat exchange between sample and surroundings. This interpretation allows these structures to be used in general in continuous wave velocity of sound and ultrasonic attenuation studies while making use of the high sensitivity of the SQUID acoustomagnetic spectrometer.

THURSDAY MORNING, 30 NOVEMBER 1989

ST. LOUIS BALLROOM BC, 8:00 A.M. TO 12:00 NOON

\author{
Session FF. Speech Communication VI: Synthesis, Perception, and Recognition (Poster Session) \\ Sigfrid D. Soli, Chairman \\ The House Ear Institute, 256 South Lake Street. Los Angeles, California 90057
}

\begin{abstract}
All posters will be displayed from 8:00 a.m. to 12:00 noon. To allow all contributors an opportunity to see other posters, contributors of odd-numbered papers will be at their posters from 8:00 to 10:00 a.m. and contributors of even-numbered papers will be at their posters from 10:00 a.m. to 12:00 noon.
\end{abstract}

\section{Contributed Papers}

FF1. Language modeling for very-large-vocabulary speech recognition. $\begin{array}{ll}\text { D. O'Shaughnessy, V. Gupta,-) M. Lennig,") F. Seitz, and } & \text {, }\end{array}$ P. Mermelstein") (INRS-Telecommunications, Nuns Island, Quebec H3E 1H6, Canada)

Virtually all recognition systems, both research and commercial, place severe limits on the vocabulary and syntactic structures a speaker may use. This recognition system is the most advanced in the world in terms of freedom from this constraint-it allows an essentially unrestricted English vocabulary and sentence structure-although it is currently required that each word be followed by a short pause. Many speech recognition systems make little use of syntactic and semantic information. A trigram language model is employed here that examines the statistical likelihood of each vocabulary word's occurrence as a function of the two preceding words. A global context-sensitive parser is also used which accepts, as input, a long sequence of word candidates. (The speaker is not required to pronounce punctuation marks.) Using rules of English syn- tax, the parser identifies many erroneous candidates and estimates locations to insert punctuation in the output text. The combined parser and trigram model have raised the accuracy of this system to $92 \%$ for ten speakers on an 86000 -word vocabulary. [Work supported by NSERC.] a) Also with Bell-Northern Research, Nuns Island, Quebec H3E 1H6, Canada.

FF2. A database demonstrating the sounds of the world's languages. Peter Ladefoged and John Choi (Phonetics Laboratory, Linguistics Department, UCLA, Los Angeles, CA 90024-1543)

A database illustrating the full range of the sounds of the world's languages would be an enormous undertaking. Consequently, this paper is restricted to trying to provide illustrations of a large number of the less 
familiar sounds. Currently, samples of about 50 languages were obtained illustrating different airstream mechanisms (!Xóõ clicks, Navaho ejectives, Igbo voiceless implosives, etc.), phonation types (Sindhi voiced aspirates, Bruu tense phonations, etc.), and places and manners of articulation (Agul epiglottal stops, Yeletnye bilabial trills, etc.). Each recording has been digitized at $22000 \mathrm{~Hz}$, with 8-bit samples, care being taken to maintain the full 48-dB signal-to-noise ( $\mathrm{S} / \mathrm{N}$ ) ratio. (However, some of the original field recordings do not have this $\mathrm{S} / \mathrm{N}$ ratio.) The database is organized as a 15-meg set of Macintosh Hypercard stacks. Clicking on a word, or a row, or column of words, reproduces the corresponding items, and makes it simple to repeat contrasting sounds. The entire stack is in the public domain, and is available (for Macintosh computers with Hypercard) at cost. [Work supported by NSF.]

FF3. LPC veetor quantization based on critical-band analysis. Daniel Lin and Brian M. McCarthy (Speech Research Department, International Mobile Machines, 2130 Arch Street, Philadelphia, PA 19103)

Critical-band spectrum is known to represent the redundancies of the human auditory system. Vector quantization constitutes a minimal redundant code set for a given signal source. In this study, these techniques are combined in a low-rate speech analysis/resynthesis system. An important design criterion for a perceptually based source coder is to define an appropriate mapping between the analysis codebook in critical-band domain and the synthesis codebook in LPC domain. Several methods for generating and searching the synthesis codebooks based on the perceptual distance criterion are proposed. Their performance with other LPC-based distance measures (e.g., log area, LSP, cepstral) is compared. These results indicate a substantial improvement in the quality of synthetic speech for the perception-based system due to smoother spectral trajectories in the low-frequency regions.

FF4. Evaluation of a glottal ARMA modeling scheme. Arthur P. Lobo and William A. Ainsworth (Department of Communication and Neuroscience, University of Keele, Keele, Staffordshire ST5 5BG, United Kingdom)

In many speech analysis/synthesis schemes, the source of excitation for voiced speech is a train of impulses. Although this is a mathematically sound scheme, there is no physiological basis for it. The quality of speech that has been attained due to the introduction of a dynamically varying source, e.g., a parametric source mode, multipulse excitation, etc., has been found to be better than that produced using impulse excitation. In this paper, a pitch synchronous glottal ARMA analysis/synthesis scheme is proposed. A parametric voice source model is discussed. The voice source and vocal tract parameters are simultaneously estimated. The AR and MA orders of the vocal tract model are variable and their values are decided every period depending on whether a resynthesis efficiency threshold is crossed during the analysis/synthesis procedure. This scheme is compared with two other schemes, viz., (1) closed phase LPC analysis/ synthesis and (2) robust LPC analysis/synthesis. The superiority of the proposed scheme over the latter two methods is shown in terms of better formant/bandwidth tracking ability and efficiency of resynthesis.

FF5. Transducer effect on automatic speech recognizer. Harry M. Chang (NYNEX, 500 Westchester Avenue, Room 1G21, White Plains, NY 10604)

It is generally recognized that the types of transducers used to input speech to automatic speech recognizers (ASR) will affect their performance. However, any ASR application over the telephone network implies the use of a large number of different telephones as input transducers to ASR. It is, therefore, very important to quantify the performance degradation due to transducer-to-transducer variation introduced in many potential ASR applications over the telephone network and identify the source of the degradation. To conduct this experiment, a database based on an 11-digit vocabulary was created from 20 native English speakers in 11 sessions in a 2 -month period. The utterances were digitally recorded on two tracks simultaneously: one from a head-mounted Shure SM-10A dynamic microphone, and the other from one of 11 selected telephones that will be different in each session. A commerical speaker-dependent recognition system was tested with both the subdatabase MIC created from the Shure SM-10A microphone and PHONE from the 11 telephones. The quantitative analysis of the evaluation tests will be presented.

FF6. A learning word-spotting method for speaker-independent word recognition in noisy environments. Hiroshi Kanazawa and Yoichi Takebayashi (Research and Development Center, Toshiba Corporation I, Komukai Toshiba-cho, Saiwai-ku, Kawasaki 210, Japan)

A learning word-spotting method has been proposed for the purpose of robust speaker-independent word recognition in noisy environments. In order to avoid word boundary detection errors at the recognition stage, the method employs word spotting based on the multiple similarity method, which was shown to be effective for noisy speech data. The learning process uses synthesized noisy speech data, a mixture of pure speech data and noise data, to design reliable word reference vectors for the word spotting. Word feature vectors with maximum multiple similarity values are automatically extracted by the word spotting. During the learning process, the signal-to-noise ratio (SNR) of the synthesized data is gradually decreased to perform word spotting accurately. Experiments were carried out for 13 words, including ten Japanese digits, spoken by 50 males. Under the condition of $10 \mathrm{~dB}, \mathrm{SNR}$ contaminated by concourse noise, the recognition scores of $85.5 \%$ and $94.1 \%$ were obtained by word spotting, without learning and with learning, respectively. The results have shown the effectiveness of the proposed method in noisy environments.

FF7. Goal-directed speech in a spoken language system. Alexander I. Rudnicky (School of Computer Science, Carnegie-Mellon University, Pittsburgh, PA 15213)

The advent of reliable speaker-independent continuous speech recognition systems has made it possible to design systems that use speech as a replacement for keyboard input. To understand the nature of a system that accepts spontaneous goal-directed speech (as opposed to the current standard of read speech), a spoken-language spreadsheet was implemented and users performing a series of tasks using this system were studied. The system was instrumented to allow the collection of detailed timing information about the components of the interaction cycle. The (HMMbased) recognition system incorporates a lexicon of 273 words and a language of perplexity 51 . Four users performed a series of 40 tasks (involving the entry of personal financial information) alternating voice and keyboard input. Users completed 30 tasks in one block of sessions, then returned a month later to complete the remainder. The utterances spoken into the system (over 7500) were stored for later analysis. The data collected provide a comprehensive picture of how users learn to use a spokenlanguage system and provide an understanding of the factors that govern performance in spoken-language systems.

FF8. The intelligibility of native and non-native speakers of American English using spelling alphabet test materials. A. Schmidt-Nielsen (Naval Research Laboratory, Code 5532, Washington, DC 20375-5000)

Male and female speakers who were native speakers of French and Dutch, as well as native American Engish speakers, were recorded reading randomized lists of the International Civil Aviation Organization (ICAO) spelling alphabet words (ALFA, BRAVO, CHARLIE, etc.) and the digits 0 to 9 . The resulting tapes were subjected to a variety of degradations, including Gaussian noise, interruption by silence and by 
noise, and LPC coding. Non-native speakers were less intelligible than the native speakers, and the decrement was greater for some of the degraded speech conditions. Results are compared with selected DRT scores for the same speakers. [Work supported by NAVSPAWARSYSCOM.]

FF9. Modeling lexical stress in read and spontaneous speech, Joseph H. Polifroni and Alexander I. Rudnicky (School of Computer Science, Carnegie-Mellon University, Pittsburgh, PA 15213)

Although prosodic information has long been thought important for speech recognition, few demonstrations exist of its effective use in recognition systems. Lexical stress information has been shown to improve recognition performance by allowing the differentiation of confusable words (e.g., Rudnicky and Li, DARPA Workshop on Speech Recogn., June 1988 ). In this study, lexical stress modeling for a spreadsheet system with significant number of confusable words (e.g., EIGHTY and EIGHTEEN) is examined. The models used here have been evaluated on both read and spontaneous speech. A database of over 400 spreadsheet and numeric utterances was available for training a (HMM-based) speaker-independent continuous-speech system with a 273-word vocabulary and language perplexity of about 51 . Testing data used in this study were based on read utterances and data generated in a separate study examining the use of a spoken-language spreadsheet. This latter set includes: (a) a "spontaneous" set, composed of parsable utterances from a spreadsheet task; (b) a "read" set, consisting of the spontaneous sentences read by their original speakers. The use of lexical stress models was found to reduce the error rate for read speech by approximately $10 \%$. A comparison with the spontaneous data will provide an insight into the nature of the improvement.

FF10. Automatic evaluation of English spoken by Japanese students. Jared Bernstein, Mitch Weintraub, Mike Cohen, and Hy Murveit (Speech Research Program, SRI International, Menlo Park, CA 94025)

The paper describes the methods and results of a study of the feasibility of automatically grading the performance of Japanese students when reading English aloud. SRI recorded 31 adult Japanese speakers: 22 men and 9 women. Each Japanese speaker read six sentences aloud. All 186 recorded utterances were presented in a random order for rating by three expert listeners who rated the utterances on two occasions. Speech-grading software was developed from an adaptive hidden-Markov-model (HMM) speech-recognition system. The grading procedure is a two-step process: First, the speech to be graded is aligned, then the segments of the speech signal that are located are compared with models of those segments that have been developed from a database of speech from native speakers of English. Important points in the results are: (1) ratings of speech quality by expert listeners are extremely reliable, and (2) automatic grades from the system correlate well $(>0.8)$ with those ratings.

FF11. Word recognition by humans and machines: Tests on a muItitalker, multistyle database. Patricia K. Kuhl, Kerry P. Green (Department of Speech and Hearing Sciences, University of Washington, Seattle, WA 98195), John W. Gordon, David L. Sanford, and Caroline Fu (Boeing Aerospace and Electronics, Seattle, WA 98124)

Experiments comparing isolated word recognition by human listners with automatic speech recognition systems are valuable because error analyses may lead to improvements in speech recognition technology. Isolated word recognition in adult human listeners has been compared with recognition performance by two commercially available speech-recognition systems. The test stimuli were drawn from the Lincoln Laboratory Stressed-Speech database. The database consists of 6930 stimuli (two iterations of each of 35 words spoken by nine different people in 11 different speaking styles). The vocabulary contains confusable words (i.e., go, hello, oh, no, and zero); the speaking styles include a wide range of natu- rally occurring variations (i.e., normal, slow, fast, soft, loud, angry). Analyses show that the acoustic characteristics of individual words vary considerably across talkers, and across styles within talkers. Performance of human listeners and the two machine-based recognition systems was tested in a single-talker, multistyle condition, and in a multitalker, multistyle condition. All tests were conducted under two listening conditions: normal, and in the presence of masking noise. The data to be presented are the error patterns of human listeners, versus the machine-recognition systems, exhibited across talkers, across speaking styles, and across training conditions (multitalker, multistyle training versus single talker, single style training ). [Work supported by Boeing Aerospace and Electronics.]

FF12. Homovocalic patterns in two- and three-syllable words: Implications for automatic speech recognition. Mary Regina Smith (Bellcore, 444 Hoes Lane, 4C-530, Piscataway, NJ 08854)

In 11067 two-syllable words and 9640 three-syllable words of the MRC Psycholinguistic Database, 5\% of the former class and $15 \%$ of the latter have the same vowel quality in two consecutive syllables. Of the 20 allowed vocalic nuclei, $95 \%$ occur homovocalically in two-syllable words. For the first two syllables of the three-syllable words, $75 \%$ of the vowel types occur homovocalically, while only $45 \%$ occur in the final two syllables of three-syllable words. Which vowels occur in these sequences is significantly affected by stress pattern, as is their relative frequency in such sequences. The likelihood that a vowel type will occur in a homovocalic sequence can be expressed as a function of quality, stress pattern, and combinatory range. The resulting patterns reduce the search space and promise improved probabilities in some automatic speech recognition strategies. [Work supported by Alvey (U.K.).]

FF13. Time and frequency spectral derivative features for robust recognition of Lombard and noisy speech. Brian A. Hanson, Ted H. Applebaum, and Gregory R. De Haan (Speech Technology Laboratory, 3888 State Street, Santa Barbara, CA 93105)

Lombard speech approximates speech variations encountered in noisy environments. This paper addresses automatic, speaker-independent recognition of Lombard and noisy speech. Spectral information was derived from production-based (LP) or perceptually based LP analysis, and represented by cepstral or index-weighted cepstral (RPS, spectral frequency derivative) coefficients. Instantaneous, dynamic (first temporal derivative), and acceleration (second temporal derivative) features were then computed from one of the representations, and integrated during the likelihood calculation of a hidden Markov model recognizer. The recognizer was trained with normal speech to evaluate its robustness to speech variations. Strong interaction was found between the temporal derivative features, the spectral derivative, and the degree of smoothing in the analysis. Although the acceleration feature performed poorly by itself, when combined with other features it generally raised recognition rates for Lombard speech. This trend was more pronounced with the cepstral representation. Combining all three features gave the best Lombard speech recognition results: The perceptually based analysis with RPS coefficients and three features yielded $94 \%$ correct recognition, compared with $63 \%$ and $82 \%$ correct for standard cepstrum and RPS, respectively, when the instantaneous feature was used alone. Experiments were also done with additive noise. The results and implications for robust speech recognition are discussed.

FF14. Speaker-independent recognition of the DARPA Naval Resource Management Task. Chin-Hui Lee, Lawrence R. Rabiner, Jay G. Wilpon, and Roberto Pieraccini") (Speech Research Department, AT\&T Bell Laboratories, 600 Mountain Avenue, Murray Hill, NJ 07974)

In this talk, the issue of acoustic modeling of the fundamental units for recognition is examined. Modeling approaches for both context indepen- 
dent (CI) and context dependent (CD) units are studied. The acoustic modeling approaches were tested on speaker independent recognition of the DARPA Naval Resource Management Task. The set of context independent units in this study is a fixed set of 47 phonelike units (PLUs), in which each PLU is associated with a linguistically defined phoneme symbol. Each CI/PLU is modeled using a continuous density hidden Markov model (CDHMM) with Gaussian mixture state observation density. The set of context dependent units includes PLUs defined by left, right, and both left and right context. Only those CD/PLUs with enough occurrences in the training data are selected for modeling. Two approaches are presented to model the CD/PLUs. Both CI/PLUs and CD/PLUs are obtained using the segmental $k$-means training procedure. In the case of context independent acoustic modeling, the maximum number of mixtures was varied in each state from 1 to 256 and it was found that the word accuracy increased from $56 \%$ to $89 \%$ which indicates that sufficient acoustic resolution is essential for improved performance. The $89 \%$ word accuracy is the highest performance reported based on context independent units. When context dependency modeling is incorporated, both modeling approaches achieved better than $92 \%$ word accuracy. ${ }^{\text {a }}$ On leave from CSELT, Torino, Italy.

FF15. Automated training for speech recognition. L. A. Smith, B. L. Scott, L. S. Lin, and J. M. Newell (Scott Instruments Corp., 1111 Willow Springs Drive, Denton, TX 76205)

A method is described for automated training of a speaker-independent isolated word recognizer. The training process generates vocabulary templates from a database of collected training utterances. These templates are then modified through adaptive training, an iterative process of testing and modifying templates in order to optimize recognition. Robustness of the templates is enhanced by varying the presentation of the collected utterances during adaptation; varying the utterance sampling rate, for example, has the effect of presenting the same utterance at differing pitches and time scales. Adaptive training continues until the error rate falls to an acceptable level. Results will be presented for similar vocabularies developed with and without adaptation and under varying adaptation conditions.

FF16. Transitional cues and redundancy in speech recognition. Brian L. Scott, Lloyd A. Smith, Lisan S. Lin, and J. Mark Newell (Scott Instruments Corporation, 1111 Willow Springs Drive, Denton, TX 76205)

Perhaps the most difficult features for speech recognition systems to properly capture and align are the transitional cues that exist between phones. There are advantages and disadvantages associated with both linear time normalization methods and dynamic time warping (DTW). Linear methods preserve the relative durations of transitional and steadystate portions of the signal but tend to smear features and lose resolution. DTW retains resolution but loses durational relations. The present algorithm attempts to compensate for the smearing associated with linear normalization by copying transitional regions of the utterance to additional locations within the representation. This process involves locating the onset and offset of the syllable nucleus based on the normalized representation and copying two frames immediately surrounding the onset and offset to another location in the representation. The process enhances performance of the recognizer in two ways. First of all, the redundancy serves to weight more heavily the perceptually important transitional region that exists between consonantal cues, such as burst frequencies, frication, nasal murmurs, etc., and the vowel nucleus of the syllable. And second, copying these regions to another location within the representation serves to enhance the alignment of these cues during training and matching. The system is being evaluated over standard telephone lines using the alphabet as the test vocabulary. The telephone environment, and the inherent confusibility of the alphabet as a test vocabulary, places sig nificant demands on the recognizer to accurately capture and identify transitional cues. The pilot study, using only a single speaker, provided encouraging results. The recognizer was $96.7 \%$ accurate over 8320 live trials. Currently, an alphabet database is being collected and speakerindependent test results will be presented.
FF17. On the use of speech recognition in articulation training Mari Ostendorf (Boston University, Electrical, Computer, and Systems Engineering Department, 44 Cummington Street, Boston, MA 02215), Kerin Gibbons (Raytheon Company, 131 Spring Street, Lexington, MA 02173), Elizabeth Gavett, and Nicholas Bankson (Boston University, Boston, MA 02215)

Computer processing of speech has been used in many ways as an aid to clinicians in various types of speech therapy. In this work, use of speech recognition for detecting articulation errors, such as a lisp or a phoneme deletion, is investigated. The main features of this approach are (1) the use of a time-dependent score in template matching and (2) the adjustment of dynamic time-warping slope constraints according to the type of articulation error (deletions versus substitutions). The use of a time-dependent score is particularly important because it allows the clinician to target a particular portion of a word for correct pronunciation. The algorithm was implemented in near real time on a PC with a DSP processor. Speaker-dependent and -independent performance was investigated for simulated and actual articulation errors. [ Work supported by Design Automation, Inc., through an SBIR grant for the National Institute for Deafness and Other Communications Disorders.]

FF18. Perception of synthetic vowels: A comparison of severa classification schemes. John W. Hawks (Central Institute for the Deaf, 818 South Euclid Avenue, St. Louis, MO 63110)

Synthetic vowel tokens (1725) were randomly presented twice to eight naive speakers of Midwestern American English for classification as one of 12 vowel categories, /IY,IH,EH,EY,AE,AA,AH,AO,OW,UH UW,ER/. Subjects rated the certainty of their responses on a scale from one (very unsure) to five (very sure). The vowels were synthesized in null context and utilized a male $F 0$ contour. The frequency values of $F 1, F 2$, and $F 3$ were selected such that the entire area where vowels may be represented in the auditory-perceputal space [ J. D. Miller, J. Acoust. Soc. Am. 85, 2114-2134 (1989)] was equidistantly sampled. The results of this experiment support the view that $F 1$ and $F 2$ are the primary determinants in the perception of nonretroflex vowels. In addition, monophthongal versions of the diphthongs /EY/ and /OW/ may be distinctly classified. The results will be presented graphically as target zones constructed on the basis of the plurality identifications for each token. These target zones are abutting and nonoverlapping, and correctly classify $99.9 \%$ of the plurality judgments (less 48 tokens where ties occurred) and $75 \%$ of the 27600 total judgments. Other vowel classification schemes will also be compared for their accuracy in classifying the results of this experiment, as well as results from other studies. [Work supported by NINCD.]

FF19. Attentional allocation during phoneme monitoring: An investigation into the unit of perceptual analysis and selective attention during speech perception. Mark A. Pitt and Arthur G. Samuel (Department of Psychology, Box 11-A Yale Station, Yale University, New Haven, CT 06520)

A variant of the phoneme monitoring technique (in which the probability with which the target phoneme occurred at a particular consonant location in a word was varied) was employed to investigate whether the phoneme or the syllable is the smallest linguistic unit of perceptual analysis, and to examine selective attention during speech perception. The results suggest that the phoneme may be a unit of perception and that subjects were able to attend selectively to a single consonant location in a word. [Research supported by AFOSR.]

FF20. On the combined use of "full" and "reduced" diphones in speech synthesis. Rob Drullman and René Collier (Institute for Perception Research, P.O. Box 513, 5600 MB Eindhoven, The Netherlands )

In the generation of synthetic speech by means of diphones, the latter are usually excised from accented syllables. Therefore, all the speech sounds that result from the concatenation of diphones have phonetic 
properties that are typical of this accented context only. In particular, their temporal and spectral characteristics are close to the target values that one finds in the production of carefully spoken, isolated, accented syllables. Speech generated by means of such diphones often sounds overarticulated. In unaccented syllables (and perhaps elsewhere, too), one might prefer to use "reduced" diphones, which may lead to shorter and spectrally less extreme speech sounds. Perception experiments were run in order to assess the improvement that can be achieved by also using "reduced" diphones, which are extracted from unaccented syllables. In these tests, the use of "reduced" diphones was determined by the following variables: word stress, sentence accent, word and phrase boundaries, metrical structure. [Research sponsored by the Dutch government as part of the national SPIN program "Speech analysis and synthesis."]

FF21. Including frequency-dependent losses in a time-domain, articulatory synthesizer. R. S. McGowan (Haskins Laboratories, 270 Crown Street, New Haven, CT 06511)

The Webster horn equation describes acoustic wave propagation in tubes with cylindrical symmetry. This differential equation can be derived from the linearized equations of motion for air neglecting viscosity and heat conduction. A modified horn equation including viscous loss in the boundary-layer approximation can be derived. This modified equation includes a convolution integral over time; thus the computational problem is to find an approximation to the integral. First, the integral is discretized, and then a rational Padé approximation is used to approximate the resulting infinite sum. The frequency dependence of this approximation is examined and compared to the ideal frequency dependence. [Work supported by NIH grants HD-01994 and NS-13617 to Haskins Laboratories.]

FF22. Excitation waveform extraction for pitch control in residualexcited LPC speech synthesis. $K$. Itoh and $H$. Sato (Speech and Acoustics Laboratory, NTT Human Interface Laboratories, Musashinoshi, Tokyo 180, Japan)

The residual-excited LPC is one of the most effective techniques for producing high-quality synthetic speech. However, this technique has difficulty controlling pitch frequency when applied to realizing synthesized speech with a pitch contour different from original speech or when applied to synthesis of arbitrary speech created with concatenated spoken units. In previous methods, pitch-period LPC residual waveforms that extracted pitch synchronously have been used to control pitch frequency. However, the extraction position $\left(E_{\rho}\right)$ and window length $\left(E_{\omega}\right)$ are very critical to synthesized speech quality, and cause voice quality deterioration. This paper proposes a new method of excitation waveform extraction that automatically determines $E_{\rho}$ and $E_{\omega}$ using spectral envelope distortion criteria between input and synthetic speech. Subjective evaluation experiments indicate that the pitch frequency pattern can be changed with a relatively small deterioration in quality. Application of this method to arbitrary speech synthesis will also be discussed.

FF23. Phonological rules for a semantics-to-speech system of Japanese: A rule application experiment. Shigeru Sato (Informatics Laboratory, Tohoku Institute of Technology, Yagiyama-kasumicho 35-1, Taihaku, Sendai 982, Japan)

In order to confirm validity of the phonological rules of Japanese and their application algorithms, a phonetic form generation experiment is performed in the phonological component of a semantics-to-speech system implemented in the computer. The rules are tiered according, first, to the nature of data they handle: segmental versus accentual, and, second, to their rule-applicational relevance to syntax: cyclic versus noncyclic.
Successful integration of segmental and accentual phases of phonological processing is shown to be possible using the concept of recursive adjunction of a suffix to a stem. The rules were installed in the computer in a human-editable format, translated by a rule compiler into executable Lisp functions, tested, and found to be valid in actual realization of synthesized speech.

FF24. On the unit set design for speech synthesis by rule using nonuniform units. Yoshinori Sagisaka (ATR Interpreting Telephonf Research Laboratories, Kyoto 619-02, Japan)

The unit set design algorithm is proposed for nonuniform synthesis units using entropy measure. This algorithm enables optimal unit set building according to statistic phonotactic characteristics without using a priori linguistic knowledge. By considering each phoneme sequence unit as a state in nonuniform order Markov process, the entropy of a unit set is defined by the entropy of a Markov information source. Each unit candidate corresponds to a new state and the new state generation corresponds to the enrollment of the candidate as a unit member. Unit enrollment takes place by finding the corresponding new state that gives the greatest decrease to the entropy of the set. Applying this algorithm to Japanese using phoneme sequence statistics derived from a Japanese word dictionary, the following characteristics were observed. (1) Linguistically wellknown CV-type syllables and diphthongs are selected in the very early stage. (2) Though the frequently used dyadic VC-type units are not selected in the early stage, the CVCV-type units that contain those VC-type units are selected in the earlier stages. (3) Many early selected units coincide with the phoneme sequence contained in morphemes. Through this experiment, it has turned out that the nonuniform unit set covered phoneme sequences about two times more efficiently than the usual uniform unit set.

FF25. Robust ARMA analysis for the determination of voice source and vocal tract control parameters in speech synthesis. Johan de Veth, Louis Boves (Department of Language and Speech, Nijmegen University, P.O. Box 9103, 6500 HD Nijmegen, The Netherlands), and Wim van Golstein Brouwers (PTT Research Neher Laboratory, Leidschendam, The Netherlands)

A cascade six pole-pair and five zero-pair synthesizer has been developed as part of a text-to-speech conversion system for Dutch. Control information for this synthesizer is derived from a.o. measurements on natural speech. A pitch synchronous robust ARMA analysis technique was developed and applied to utterances produced by a number of adult male talkers. The resulting pole-zero parameters were separated into sets pertaining to the source of the vocal tract. The vocal tract parameters were corrected in those frames where the analysis method made occasional mistakes. Analysis-resynthesis of sentence material using the corrected vocal tract parameters to control the synthesizer driven by impulse and noise excitation yielded high-quality synthetic speech. The tract parameters were then used to inverse filter the speech, to obtain the source function, that was subsequently parametrized using the LiljencrantsFant model. It is hoped that the speech quality will be improved by replacing the impulse excitation by the controllable source model.

FF26. Restoration of tracheoesophageal voice with LPC resynthesis. Yingyong Qi (Department of Speech and Hearing Science, University of Arizona, Tucson, AZ 85721)

Four vowels, [i], [æ], [a], and [u], and one diphthong [ou], produced by two male and two female tracheoesophageal speakers, were analyzed with the LPC autocorrelation method. The vowels were synthesized by replacing the original source with an impulse train. The fundamental 
frequency of the impulse train was $100 \mathrm{~Hz}$ for the male and $200 \mathrm{~Hz}$ for the female speakers. The results of an identification experiment indicated that both the vowel and the gender of the speaker can be better identified from the synthesized vowels than from the original ones. The possibility of improving the quality of tracheoesophageal speech and building prosthetic device with the LPC technique will be discussed.

FF27. Effects of spectral smearing on speech perception. Mariken ter Keurs, Joost M. Festen, and Reinier Plomp (Department of Otolaryngology, Free University Hospital, P.O. Box 7057, 1007 MB Athsterdam, The Netherlands)

Connected speech presented in quiet is highly redundant. For such a condition, the resolution of spectral contrasts by the ear seems to be much larger than would be required. This suggests that the ear's high selectivity in frequency is particularly important to understanding speech in the presence of interfering sounds. The effect of reduced frequency resolution on the speech-reception threshold (SRT) for sentences in noise was investigated for eight normal-hearing subjects by simulating an auditory system with variable frequency selectivity. Signal processing was performed by short-time fast Fourier transforms (FFT), reduction of contrast in the spectral envelope without affecting the harmonic structure, and overlapping additions to reconstruct a continuous signal. The spectral envelope in the frequency region from 100 to $8000 \mathrm{~Hz}$ was smeared over fixed relative bandwidths of $1 / 8,1 / 4,1 / 2,1,2,4$, and 8 octaves. Results show that the SRT increases progressively as the spectral envelope is smeared over bandwidths exceeding the ear's critical bandwidth. In a second experiment phoneme, confusions as a result of three different degrees of spectral smearing are studied in nonsense CVC syllables.

FF28. Perception of vowels by budgerigars (Melopsittacus undulatus). Robert J. Dooling, Susan D. Brown, Amy Nespor (Department of Psychology, University of Maryland, College Park, MD 20742), and John W. Hawks (Central Institute for the Deaf, St. Louis, MO 63110)

Budgerigars (parakeets) were trained using operant conditioning techniques to respond to differences between speech stimuli. Response latencies were used to construct similarity matrices and multidimensional scaling procedures were then used to produce perceptual (spatial) maps of these stimuli. For natural vowel tokens, budgerigars showed evidence of perceiving phonetic categories in spite of variation in talkers including talker gender and talker age. Experiments with synthetic vowel tokens generally confirmed and extended these findings. Multiple regression techniques revealed that the perceptual dimensions obtained from MDS were highly correlated with the frequencies of the first and second formants. These results suggest that both natural and synthetic vowels are probably perceived in similar ways by budgerigars and humans. These results have relevance for theories of speech perception and language development in humans. [Work supported by NIH.]

FF29. Articulation index importance functions for contextual speech materials. Theodore S. Bell, Donald D. Dirks, and Timothy Trine (UCLA School of Medicine, Head \& Neck Surgery, Rehabilitation Building, Room 31-24, Los Angeles, CA 90024-1794)

The relative importance of one-third octave frequency bands toward the intelligibility of speech in various contexts was examined. Thirty-five young normal hearing adults heard sentences in which the final word ( target stimulus) was either predictable (probability-high; $\mathbf{P H}$ ) or unpredictable (probability-low; PL) from the context of the sentence. Sentences were presented at $\mathrm{S} / \mathrm{N}$ 's from -8 to $+14 \mathrm{~dB}$ in a noise shaped to conform to the peak spectrum of the speech, and crossover frequencies as related to the articulation index (AI) were determined by successively high-pass and low-pass filtering the stimuli to bisect intelligibility. Results indicated only slight difference in $1 / 3$ oct importance functions as the result of context (PH vs PL), although the crossover frequency was significantly different and showed no interaction attributable to the noise. The effect of context in these speech materials was related to differences in perceptual dynamic range more so than the frequency importance func- tion. These results are contrasted to other recent studies, and methodological and theoretical aspects of parameter estimation in the AI model are discussed.

FF30. An algorithm for distinguishing between voiced stops and voiced fricatives. LaDeana F. Weigelt, Steven J. Sadoff, and James D. Miller (Central Institute for the Deaf, 818 South Euclid Avenue, St. Louis, MO 63110)

An algorithm has previously been reported which distinguished voiceless stops from voiceless fricatives with a success rate of $96.8 \%$ [J. Acoust. Soc. Am. Suppl. 1 85, S56 (1989) ]. With only slight modifications, this algorithm also makes the voiced stop/fricative distinction. Here, results are presented on the modified algorithm. The input signal is high-pass filtered (cutoff frequency of $125 \mathrm{~Hz}$ ) and two measures of the resulting waveform are used: the rms energy and the derivative of rms energy over time (termed rate of rise, ROR). The ROR is used as the primary classifier while energy pulse duration and relative level are used to discard spurious, irrelevant peaks. Peaks in ROR are considered in order of magnitude for relevance to the stop/fricative distinction. The resulting algorithm was tested on $420 \mathrm{CVC}$ tokens (three male speakers, three female speakers, three stops and an affricate $[B, D, G, J H]$, four fricatives $[\mathrm{Z}, \mathrm{ZH}, \mathrm{V}, \mathrm{DH}]$, and ten vowel contexts [IY,IH,EH,AE,AA,AH,AO, UH,UW,ER]) recorded in an anechoic chamber. Data from two male and two female speakers (280 tokens) were used as a training set, and the remaining data ( 140 tokens) were used as a test set. The overall success rate was $97.9 \%$. [Work supported by AFOSR.]

FF31. The role of speech rate in social evaluation. Cynthia $L$. Crown (Department of Psychology, Xavier University, Cincinnati, OH 45207)

Prior studies concerned with the relation of speech rate to the personality variable of extraversion and to social evaluation (i.e., interpersonal perception) have primarily examined monologues. The study reported here was designed to (a) explore such dependencies in dialogues, and (b) investigate the possibility that speech rate may be influenced by differences in interpersonal attraction. The 38 female pairs who participated in the study were selected on the basis of a sociogram designed to assess their attraction to each other prior to their participation in the study. Their sociogram scores divided them into three groups: those who liked each other, those who disliked each other, and those who were unacquainted with each other. Each pair engaged in a 30-min conversation from which the speech rate of each participant was electronically determined. The result of multiple regression analyses indicated that those women who spoke more quickly perceived themselves more positively, whereas those who spoke more slowly viewed their partners more positively. However, variations in rate were related to neither interpersonal attraction nor extraversion.

FF32. Two paradigms for examining the role of phonological stress in sentence processing. David W. Gow, Jr. and Peter C. Gordon (Department of Psychology, Harvard University, Cambridge, MA 02138)

The role of phonological stress in sentence processing was studied using a syllable monitoring task, as well as a new short-term memory probe task. In both tasks, target syllables were embedded in bysyllabic noun/verb homophones with syntactic category-dependent stress patterns. The stress and position within a word of target syllables were manipulated. Syntactic context was also manipulated to examine the role of syntactic constraints on the anticipation of stressed syllables. The syllable monitoring task reproduced the familiar facilitation of stressed syllable detection, and showed an effect of syntactic constraint. Stress facilitation was not found when targets were word-final. These results were interpreted in terms of stress-facilitated lexical segmentation and access processes. The short-term memory probe task introduced in this research was shown to be sensitive to stress, but not to other sentential factors that were examined. The application of this paradigm to issues in metrical phonology and short-term memory is discussed. [Work supported by AFOSR.] 


\title{
Session GG. Psychological Acoustics VI, Physiological Acoustics II, and Speech Communication VII: Sensory Aids for the Hearing Impaired
}

\author{
Margaret W. Skinner, Chairman \\ Otolaryngology, McMillan Hospital 9901, Washington University, St. Louis, Missouri 63110
}

Contributed Papers

8:30

GG1. Results in children using the 22-electrode cochlear implant. $P$. W. Dawson, P. J. Blamey, G. M. Clark, P. A. Busby, L. C. Rowland, S. J. Deltman, A. M. Brown, R. C. Dowell, F. W. Rickards, and J. I. Alcantara (Human Communication Research Centre, Department of Otolaryngology, University of Melbourne, Parkville, Victoria 3052 and The Cochlear Implant Clinic, The Royal Victorian Eye and Ear Hospital, 32 Gisborne Street, East Melbourne, Victoria 3052, Australia)

Five children (aged 6.0 to 14.8 years) out of a group of nine implanted with the 22-electrode cochlear implant (Cochlear Pty. Ltd.) have achieved substantial open-set speech recognition scores for monosyllabic word ( $30 \%$ to $72 \%$ for phonemes) and sentence ( $26 \%$ to $74 \%$ for words in sentences) tests using hearing without lipreading. Four of these five children were implanted during preadolescence and the fifth, who had a progressive loss, was implanted during adolescence. The remaining children who did not demonstrate open-set recognition were implanted during adolescence after a long duration of profound deafness. Post-operative performance on closed-set speech perception tests was better than preoperative performance for the group of five children with open-set recognition. Improvements in speech and language assessments were also noted for these children. The results are discussed with reference to variables that may contribute to successful implant use, such as age of onset of loss, duration of profound loss, age at implantation, aetiology, educational program, and the type of training provided.

\section{8:45}

GG2. Psychophysical studies on cochlear implant patients deafened prior to 4 years of age. P. A. Busby, Y. C. Tong, G. M. Clark, and J. I. Alcantara (Department of Otolaryngology, University of Melbourne, Parkville, Victoria 3052, Australia)

Psychophysical studies were conducted on eight prelingually deaf cochlear implant patients, between 5 and 23 years of age at the time of surgery, who were deafened prior to 4 years of age. Electrical stimulation of the auditory nerve was by means of the Nucleus multiple-electrode prosthesis. The average difference limens for the prelingual patients in electric current level (7\% of the dynamic range between threshold and comfort level), duration ( $17 \mathrm{~ms}$ for a standard of $30 \mathrm{~ms}$ ), and the detection of a gap in a $1-\mathrm{s} \mathrm{signal}(9 \mathrm{~ms})$ were in the same range as those reported for postlingually deafened adult implant patients. The average difference limens in repetition rate for the prelingual patients $(19 \%$ for a standard of $100 \mathrm{rep} / \mathrm{s}$ and $28 \%$ for a standard of $200 \mathrm{rep} / \mathrm{s}$ ) were poorer than those found for the postlingual patients $(5 \%$ for $100 \mathrm{rep} / \mathrm{s}$ and $8 \%$ for 200 $\mathrm{rep} / \mathrm{s}$ ). Individual differences in performance were observed for the prelingual patients in all studies.
Seven prelinguistically profoundly deaf children between 7 and 11 years of age were trained in the perception of vowel duration and place, the fricative $/ \mathrm{s} /$, and manner of articulation $(/ \mathrm{m} / \mathrm{vs} / \mathrm{b} /$, and $/ \mathrm{s} / \mathrm{vs} / \mathrm{t} /)$ distinctions in the tactile-plus-hearing (TA) and tactile (T) conditions. Speech feature recognition tests were conducted in the TA, T, and hearing (A) conditions during the following: pre-training, during-training, and post-training phases. Test scores in the TA and $T$ conditions were signifcantly greater than $\mathrm{A}$ condition scores for all but one test, suggesting that the addition of the tactile supplement improved the perception of these features. During- and post-training phase test scores in the TA and T conditions were significantly greater than pre-training scores suggesting that the training given was responsible for the improvement in feature perception. During- and post-training scores were similar suggesting that perceptual skills acquired during training were retained after training had been removed.

\section{9:15}

GG4. A pitch perception study for a cochlear implant user with residual hearing in the contralateral ear. R. van Hoesel and Y. C. Tong (Department of Otolaryngology, University of Melbourne, Parkville, Victoria 3052, Australia)

In order to combine electrical and acoustic signals for multichannel cochlear implant users with residual hearing in the unimplanted ear, it is important to determine the degree of overlap in pitch sensations on the two sides. A pitch scaling experiment was performed maintaining constant and identical repetition rates on both the implanted (left) and normal (right) ear. The acoustic signals consisted of bandpass-filtered pulses (with center frequencies ranging from $350-850 \mathrm{~Hz}$ ). These were transmitted in synchrony with electrical pulses to one of five selected bipolar electrode pairs spanning $15.75 \mathrm{~mm}$ of the scala tympani (from approximately 2.25 to $18 \mathrm{~mm}$ beyond the base). The spacing between the two electrodes of each bipolar pair was $1.5 \mathrm{~mm}$. The experiment was repeated for repetition rates of 112.5 and $205 \mathrm{~Hz}$. Pitch sensations on the left (electrical) side were reported to be as low as, and at times even lower than, on the right (acoustic) side. All signals were scaled with respect to an acoustic reference stimulus that had a bandpass filter center frequency at $\mathbf{4 5 0}$ $\mathrm{Hz}$ Synthetic vowels were derived from the pitch scaling results and acoustic strategies only as well as combined electrical and acoustic strategies were tested. It was found that the best results were obtained from a combined electrical and acoustic strategy where the electrode information is chosen such that pitch perception overlap with the acoustic side is minimized. [Work supported by NIH Grant NS21027.]

9:30

GG5. Loudness summation study on multielectrode pair stimulation. $H$. H. Lim, Y. C Tong, R. D. Hollow, A. E. Vandali, and R. V. Hoesel (Department of Otolaryngology, University of Melbourne, Parkville, Victoria 3052, Australia)

This psychophysical study investigated loudness summation for the activation of two, three, or four electrode pairs in a stimulus period. The electrode pairs were activated in temporally nonoverlapping manner. 
Two multichannel cochlear implant patients participated in the study. The method of magnitude estimation was used. Loudness growth for each electrode pair was first determined. The loudnesses of each electrode pair in a multielectrode pair stimulus were always set equally loud. The results showed that: (a) loudness increased with current level and the number of electrode pairs within a stimulus; (b) loudness summation for stimuli containing two electrode pairs was complete when the electrode pairs were approximately four electrodes apart $(3.00 \mathrm{~mm})$; and (c) loudness summation for stimuli containing three or four electrode pairs was incomplete in comparison to the results obtained for stimuli containing two electrode pairs. [ Work supported by NIH Contract NO1-NS-9-2400 and NIH Grant NS21027.]

\section{9:45}

GG6. Perceptual independence of pulse rates used in speech coding schemes for a multichannel cochlear implant. W. K. Lai, Y. C. Tong (Department of Otolaryngology, University of Melbourne, Parkville, Victoria 3052, Australia), J. B. Millar (Research School of Physical
Sciences, Australian National University, Canberra 2601, ACT, Australia), G. M. Clark, and R. van Hoesel (Department of Otolaryngology, University of Melbourne, Parkville, Victoria 3052, Australia)

Two of the most important electric signal parameters for presenting useful acoustic speech information to the University of Melbourne/ $\mathrm{Nu}$ cleus multichannel cochlear implant are electrode position and electric pulse rate. The possibility of using pulse rate to encode acoustic speech information was investigated in this study. A set of $16(4 \times 4)$ orthogonal stimuli comprising all possible combinations of four different pulse rates on each of two fixed bipolar electrode pairs was presented to three cochlear implant recipients for identification. Tests for perceptual independence in terms of information transmission indicate that the two pulse rates presented on each of the two electrode pairs were perceived as partially separable dimensions. It may therefore be possible to use individual pulse rates on different electrode pairs in a speech coding scheme to present further acoustic speech information to a multichannel cochlear im. plant. [Work supported by NIH Contract NO1-NS-9-2400 and NIH Grant NS21027.]

\section{0:15}

GG7. Test materials for evaluating speech perception ability of cochlear implant patients. Yumiko Fukuda (Research Institute, National Rehabilitation Center for the Disabled, Namiki 4, Tokorozawa 359, Japan)

A system for assembling the test materials that are used in evaluating speech perception ability by combined use of cochlear implant and lipreading has been developed. In order to construct balanced lists, a database of Japanese basic vocabulary furnished with specifications regarding their auditory, visual, temporal, and linguistic components is utilized in this system. Factors whose distribution are balanced between each other list are the kind of vowel and the manner/place of articulation of the consonant, special Japanese syllables (elongated vowel, choked sound, and syllabic nasal), phones having the same mouth shape, number of syllables in a word and phrases in a sentence, class of familiarity, and grammatical data such as part of speech. All these data and specification of category of meaning of words in the database are used in searching suitable words for the lists, as well as in analyzing characteristics of perception ability of the patients. Problems in requirements for the evaluation methods, construction of balanced lists from the database, and video recordings of speech and mouth shape of utterances of the lists are discussed in this paper.

\section{0:30}

GG8. A gating task to measure the contribution of contextual information in speech perception by cochlear implant wearers. Teresa $A$. Zwolan and Thomas D. Carrell (Children's Memorial Hospital, Department of Communicative Disorders, Chicago, IL 60614 and Northwestern University, Department of Communication Sciences and Disorders, Evanston, IL 60202)

Contextual cue utilization by two groups of cochlear implant wearers was measured with a gating task. Group 1 consisted of implant wearers who were able to perceive open-set speech (unpracticed, unfamiliar words and sentences), while group 2 consisted of implant wearers who were unable to perceive open-set speech. Gated stimuli consisted of high- and low-predictability sentences taken from the speech perception in noise (SPIN) test. Subjects' responses were evaluated to determine the amount of acoustic information required for phoneme and word recognition as well as the percentage of phonemes and words identified in each type of context. Results indicated that open-set listeners required less informa- tion than non-open-set listeners to recognize phonemes and words. More importantly, a predictability-by-listener interaction demonstrated that open-set listeners were better able to make use of contextual cues than were non-open-set listeners.

\section{0:45}

GG9. The effect of varying the amplitude-frequency response on the masked speech-reception threshold for sentences in conditions with lowfrequency noise. Janette van Dijkhuizen, Joost $M$. Festen, and Reinier Plomp (Department of Otolaryngology. Free University Hospital, P.O. Box 7057, 1007 MB Amsterdam, The Netherlands)

Within a study on the merits of a frequency-dependent automatic gain control in hearing aids, the beneficial effect of adapting the amplitudefrequency response to situations of seriously interfering low-frequency noise is studied. For 12 normal-hearing and 12 hearing-impaired listeners, the masked speech-reception threshold (SRT) for sentences is measured. The noise has a spectrum identical to the long-term average spectrum of the sentences, but with its level in one octave band raised by a constant 20 $\mathrm{dB}$ in the first experiment, and slowly increasing by $20 \mathrm{~dB}$ during sentence presentation in the second experiment. Results indicate that, in conditions with steady-state noise, selective attenuation of the signal in the band containing the extra noise gives an improvement of speech intelligibility in terms of speech-to-noise ratio of up to about $4 \mathrm{~dB}$ for both the normalhearing and hearing-impaired group. In conditions with time-varying noise, a similar improvement was observed for both groups.

\section{1:00}

GG10. Simplified tones for profoundly deaf tone-language speakers. Teresa Ching (Department of English, Chinese University of Hong Kong, Shatin, N.T., Hong Kong)

The larynx frequency pattern of speech contributes significantly to speech communication when it is dependent on lipreading, especially in a tone-language context. Tests using syllable-based pitch contrasts as well as connected speech in controlled interactive situations show that receptive skills can be considerably improved both in Mandarin and Cantonese (two Chinese dialects). On the basis of findings which show that a sinusoidal presentation of voice pitch changes is more beneficial to a pro- 
foundly deaf person than the complete acoustic signal [ S. Rosen and A. J. Fourcin, Speech, Hearing and Language, U.C.L. No. 1 (1983) ], the present work adopts this simplification approach in an attempt to enhance prosodic skills in profoundly deaf Cantonese children. Results indicate that productive and perceptual skills can be significantly enhanced by using a simplified auditory presentation. These have important implications for the design of signal processing aids and the development of speech skills in the profoundly deaf tone-language speaker.

\section{1:15}

GG11. Speech feature encoding through an electrotactile speech processor. Robert S. C. Cowan, Peter J. Blamey, Joseph I. Alcantara, Lesley A. Whitford, Graeme Clark (Department of Otolaryngology, University of Melbourne, Parkville, Victoria 3052, Australia), and Geoff Plant (National Acoustics Laboratories, Sydney, NSW, Australia)

Efficiency of speech feature encoding by the University of Melbourne multichannel electrotactile speech processor was assessed through performance of eight subjects on a closed-set tactual test battery. The battery consisted of 12 subtests, each assessing discrimination of a particular speech feature in an ABX paradigm. All subjects scored significantly above chance on suprasegmental features such as syllable number, stress, and vowel length. Discrimination of vowel formant contrasts was significantly above chance for seven subjects. Discrimination of consonant manner varied across specific subtest contrasts, with better encoding of the high-frequency fricatives and affricates. Discrimination of initial voicing contrasts suggested that tactual encoding of this feature could be improved. Comparison of these results with test battery scores from five different subjects with five commercially available tactile devices showed superior encoding of vowel formant and manner contrasts by the Tickle
Talker. Subsequent consonant confusion studies with two hearing-impaired adults, one using tactual input in combination with lipreading and one in combination with aided residual hearing and lipreading, confirmed that added tactual information reduced the proportion of manner confusions and to a lesser extent voicing confusions.

\section{1:30}

GG12. Evaluation of a 32-channel electrotactile vocoder. Kathleen Rakowski, Christine Brenner, and Janet $M$. Weisenberger (Central Institute for the Deaf, 818 S. Euclid, St. Louis, MO 63110)

Previous evaluations of laboratory prototype multichannel tactile aids have shown them to provide useful speech information. However, few of these prototypes have been successfully converted into wearable devices. The present study evaluated a device that has recently become commercially available, the Audiotact (Sevrain-Tech, Inc.), a 32-channel electrotactile vocoder wom on the abdomen. Four subjects participated in the study, two of whom had previous experience with other tactile aids. In addition, a visual display designed as a training aid for new users of the Audiotact was evaluated. This display provides a visual representation of the activity on the tactile array. Two of the subjects were instructed to use this visual information while wearing the Audiotact. Results of testing with minimal pairs and small set phoneme identification tasks showed that manner features were transmitted quite well. Subjects were also able to integrate information available through lipreading with information from the Audiotact. However, preliminary results for connected discourse tracking with the Audiotact showed only a small improvement over lipreading alone. The results of this study are discussed in terms of comparability to other multichannel tactile devices.

\title{
Session HH. Structural Acoustics and Vibration IV: Structural Vibration and Acoustic Radiation
}

\author{
Jerry H. Ginsberg, Chairman \\ School of Mechanical Engineering, Georgia Institute of Technology, Atlanta, Georgia 30332
}

Chairman's Introduction-8:30

\section{Contributed Papers}

\section{$8: 35$}

HH1. Axisymmetric vibration of spheroidal shells for high-aspect ratio. Pei-Tai Chen and Jerry H. Ginsberg (School of Mechanical Engineering, Georgia Institute of Technology, Atlanta, GA 30332)

Assumed mode expansions based on Hamilton's principle were employed in an earlier presentation [P. T. Chen and J. H. Ginsberg, I. Acoust. Soc. Am. Suppl. I 85, S139 (1989)] to study fluid-structure interactions of a submerged prolate spheroidal shell of low-aspect ratio. In that approach, a straightforward analysis of the in vacuo shell vibration properties was found to be efficient for the lower and upper branches of axisymmetric deformation, provided that the aspect ratio of the spheroid is not large. The present paper addresses methods by which the first few modes of both branches may be determined for cases of high-aspect ratio. In order to overcome numerical ill-conditioning, an iterative variational method is employed to seep the natural frequencies and mode shapes with increasing mode number. In addition, in order to ensure convergence to the upper branch, which oocurs in a much higher frequency band that that of the lower branch, an artificial potential energy affecting only high wavenumbers is added. The model data derived in this manner are used to analyze a case of forced vibration of a submerged shell. The results are compared with Yen and Dimaggio's finite difference solution [J. Acoust. Soc. Am. 41, 618-626 (1967)]. [Work supported by the Office of Naval Research, Code 1132-SM.]

\section{8:50}

HH2. Response of an infinitely long cylindrical shell with a convected internal fluid. G. Leyrat and J. M. Cuschieri (Department of Ocean Engineering, Florida Atlantic University, Boca Raton, FL 33431)

The response of an infinitely long cylindrical shell excited by a point force, with an internal heavy medium (water) moving with a constant flow speed is examined. The solution is obtained by linearizing the differential equations describing the behavior of the fluid and the structure by using a spatial Fourier transform in the axial direction and a modal decomposition in the circumferential direction. The inverse Fourier transform of the solution of the linear set of equations gives the coupled solution for the shell/fluid system. Using this solution, input and transfer 
mobility functions are determined for a copper pipe of radius $0.025 \mathrm{~m}$ and wall thickness $1.5 \mathrm{~mm}$. For the input mobility functions, the nearfield effects combine with the coriolis effects of the moving fuid to produce instabilities of the circumferential modes at velocities that depend upon the mode number. For the transfer mobility functions, broadband peaks appear caused by the phase lag between waves of different circumferential mode numbers. These peaks are shifted and modified as the flow speed changes.

\section{9:05}

HH3. Response of a fluid-filled spherical shell submerged in an infinite fluid medium to a transient acoustic wave. Thomas $L$. Geers and Peizhen Zhang (Department of Mechanical Engineering, University of Colorado, Boulder, CO 80309)

Transient response problems involving fluid-filled shell structures submerged in infinite fluid media arise in various fields, including medicine, defense, and materials engineering. A canonical problem of this class possesses a spherical geometry, for which no solutions apparently exist. This paper delineates a relatively simple formulation and method of solution for this canonical problem and presents solutions for excitation by an incident step wave. The formulation begins with the familiar equations of motion for a thin spherical shell and the wave equation for the internal and external fluid domains. The Laplace transform is invoked, and the usual separation of variables method yields modal expressions involving Legendre polynomials and modified spherical Bessel functions. The explicit expressions for the latter are then manipulated to yield, after transform inversion, delayed differential equations in time for each response mode of the shell-fluid system, which are integrated numerically in time. Complete response solutions then follow by modal superposition, with special techniques being employed to improve modal convergence. To validate the methodology, numerical results are first presented for an incident step wave exciting a shell with mass density and acoustic velocity identical to the corresponding properties characterizing the same internal and external fluid. Results are then shown for a step-wave-excited steel shell containing water and submerged in water. [Work supported by DNA.]

\section{9:20}

HH4. Active control of panel-radiated noise using multiple piezoelectric actuators. B. T. Wang, E. K. Dimitriadis, and C. R. Fuller (Department of Mechanical Engineering, Virginia Polytechnic Institute and State University, Blacksburg, VA 24061)

The potential for active control of structurally radiated nosie by twodimensional piezoelectric patches bonded to the surface of thin elastic panels is analytically investigated. A previously presented approximate model for the loads induced by piezoelements bonded to the plate surface is employed for the calculation of the vibration and sound radiation from simply supported rectangular panels. The piezoelectric action modifies the vibration and hence effects the noise radiated by the panel when excited by constant distributed external forces. The complex amplitude of the voltage applied to the piezoelectric is estimated via an optimization scheme that minimizes the total radiated acoustic power into the farfield. Results have been obtained for a single as well as for multiple independently controlled actuators. It is seen that quite significant control is possible in some cases with a single appropriately taylored actuator. The effectiveness, however, deteriorates as control of higher vibration modes is attempted. Much better results are achieved with two or more independently controlled actuators bonded to the plate. It is clearly shown that multiple, appropriately positioned actuators drastically reduce undesired control spillover to the residual modes. [Work supported by ONR.]

\section{9:35}

HH5. Coupling effects between a lumped vibrational source of finite impedance and a plane radiator. Dominique Trentin, A. Berry, and Frederic Laville (GAUS, Mechanical Engineering, University of Sherbrooke, Sherbrooke, Quebec JIK 2R 1, Canada)
A typical situation in structural acoustics and vibrations is the case of a mechanical source of vibrations attached to a large, thin structure (radiator ). In general, the force input into the structure, responsible for undesired vibrations of, and radiation from, the structure, is the resultant of both the output impedance of the source and the input impedance of the structure. A theoretical analysis of the problem is presented in the case of a lumped mechanical source composed of masses, springs, and dampers, with one single attachment point to a baffled, thin, rectangular, plane structure. The coupling assumes only pure transverse force transmission into the structure. A quadrupole approach for the source assembly and a variational approach for the motion of the structure allow, respectively, the source output impedance and the structure input impedance to be calculated in a variety of configurations. The force input into the structure, kinetic energy of the structure, and radiation of sound from the structure can then be derived. It is shown how the natural frequencies of the combined system can be found from the natural frequencies of the source and structure decoupled. Numerical results are presented for the particular application of optimal suspension design of mass-type source. [Dr. I. Michaluk is acknowledged for fruitful discussions.]

\section{9:50}

HH6. Active control of flexural power flow in beams using piezoceramic actuators. Gary Gibbs and Chris Fuller (Department of Mechanical Engineering, Virginia Polytechnic Institute and State University, Blacksburg, VA 24061)

Previous research has demonstrated that the flexural power flow in thin beams can be attenuated using active point force inputs [C. R. Fuller and L. O. Gonidou, "Active vibration control of flexural power flow in beams," J. Acoust. Soc. Am. Suppl. 1 84, S47 (1988) ]. In this paper, the results of experiments that demonstrate the feasibility of using surfacebonded piezoceramic actuators and sensors in controlling vibrational power flow in elastic structures are presented. The control system utilizes both a piezoceramic control actuator and sensor, and a time domain leastmean-squares adaptive algorithm to minimize transmitted power flow. Both semi-infinite and finite beams of various geometries are considered, and the results show significant reduction of vibrational power flow using only a single piezoceramic transducer as the control actuator. [Work supported by NASA Langley Research Center.]

\section{0:05}

HH7. Vibration and stability of an elastic beam subjected to a periodic axial load with time-dependent displacement excitation at both ends. Xiao-Feng $\mathrm{Wu}_{\mathrm{u}}$ and Adnan Akay (Department of Mechanical Engineering, Wayne State University, Detroit, MI 48202)

This paper considers the vibration and stability of an elastic beam under the excitation of a combined periodic axial load and time-dependent displacement at its ends. Such problems are often encountered in the prediction of noise radiation from high-speed planar mechanisms with elastic couplers. The governing equation of motion for the vibrating beam derived by the Hamilton's principle is highly nonlinear. To obtain an approximate solution, the method of assumed modes is used. The equation of motion then reduces to a Hill type of equation in time. The parametric studies of the stability of the beam is carried out. The stable and unstable regions of the beam vibrations under the time-dependent displacement excitations are investigated. The independent restraint motions at the ends of the beam are described by the displacement infuence functions. The total response is finally obtained by adding the relative motion of the beam under the given boundary conditions to the specified displacement at its ends.

10:20

HH8. Nonlinear effects in a driven vibrating wire. Roger $J$. Hanson and Hilliard K. Macomber (Physics Department, University of Northern Iowa, Cedar Falls, IA 50614-0150) 
A horizontal wire is driven sinusoidally with a vertical magnetic force. Horizontal and vertical optical detectors follow the motion of a point on the wire. With a moderately large driving force, the usual predicted nonlinear effects are observed as the driving frequency is increased from below resonance. These include the onset of a horizontal component of motion, phase shifted $90^{\circ}$ with respect to the vertical component, and ultimately resulting in circular motion of the wire. However, with a much weaker driving force, the phase relation between the horizontal and vertical components is no longer fixed at $90^{\circ}$ and becomes very frequency sensitive in a certain range. It appears to vary from $180^{\circ}$, producing inclined straight-line motion, to $90^{\circ}$, to $0^{\circ}$, again producing straight-line motion but with a positive slope. Further increase of the driving frequency causes the vertical excursion of the straight-line motion to increase while the horizontal excursion changes little. A second harmonic in the horizontal component becomes pronounced and leads to figure-eight motion. These effects appear not to have been predicted and will be illustrated on videotape.

\section{0:35}

HH9. Analysis of sound transmission through flexible panel/frame walls. Donald B. Bliss (Department of Mechanical Engineering, Duke University, Durham, NC 27706)

Low-frequency sound transmission through flexible panel/frame walls is an important noise control problem, particularly for propeller aircraft interior noise. The coupled structural/acoustic problem for spatially periodic structures is solved using a multiple-scale perturbation expansion, based on the scale separation between panel size and acoustic wavelength, and an averaging procedure applied over the rapidly varying (panel) scale. A composite solution is formed from a smoothed global solution and a periodic local solution that accounts for the detailed panel motions. The panel subsystems appear in an averaged sense in the global solution through transfer functions, obtained from the local solution for distributed frame loadings and acoustic boundary conditions. Walls composed of identical panel subsystems, each containing several different panels, attached to a flexible frame, can be analyzed. This arrangement allows for noise reduction by alternate resonance tuning (ART), in which adjacent panels are tuned to resonate alternately above and below the frequency to be attenuated, with the resulting out-of-phase motion producing acoustic cancellation. Solutions are presented for a model problem involving sound transmission through a panel/frame wall and compared with the exact solution to assess the accuracy of the perturbation method. Noise reduction by ART is demonstrated by using two panels with different resonant frequencies in each subsystem. [Work supported by NASA Langley Research Center.]

\section{0:50}

HH10. Acoustic radiation of stiffened shells covered with a layer of slightly compressible material. B. Laulagnet and J. L. Guyader (Laboratoire Vibrations-Acoustique INSA de Lyon, Bât. 303-20, avenue Albert Einstein, 69621 Villeurbanne Cedex, France)

This work deals with the calculation of sound radiation from a shell covered with a layer of slightly compressible material and immersed in water. In this case, the assumption of locally reacting material is unrealistic, and a more elaborate model must be used to describe the behavior of the coat ing layer. This has been achieved by solving the equations of elasticity in cylindrical coordinates, and by use of asymptotic expansions. The layer displacements and stresses are then coupled to the shell displacements and fluid loading on the boundary surfaces. The calculation of shell radiation is made using the normal modes of the in vacuo basic shell, and the asymptolic expansion truncated to the second order. Numerical results are presented showing the influence of layer thickness, damping, and Poisson ratio. [Work supported by D.R.E.T. and C.E.R.D.A.N., Ministère de la Défense.]
HH11. A new efficient Ritz vectors technique to solve elastoacoustic problems. J. P. Coyette (Dynamic Engineering NV, Research and Development, Ambachtenlaan 21, B-3030 Heverlee, Belgium)

Solution of interior elastoacoustic problems in the frequency domain is addressed through an efficient nonmodal approach. The procedure relies on a symmetric two-field formulation (displacement variables are selected for the structure, while velocity potential is used for the acoustic medium). The related second-order system is transformed into a firstorder one by doubling the size of the system. An updated Ritz vectors process allows the generation of two coupled bases, which enables the accurate description of both structural and acoustic responses. Details about the generation of these vectors are provided. The process requires only the solution of the static problems and the performance of basic orthogonalization and scaling operations. The resulting high-vectorization ability is stressed. Comparisons with conventional modal synthesis procedures are provided in order to show the efficiency of the proposed approach. It is shown that the selection of this technique leads to an optimal solution scheme. Various numerical results related to Guy's problem (cavity backed plate) are reported. Extensions to deal with structural and/or acoustic damped cases are also reported.

\section{1:20}

HH12. Separation of a one-dimensional bending wave field into propagating and standing parts based on the reflection coefficient estimation. Jukka Linjama and Tapio Lahti (Technical Research Centre of Finland, VTT/INS, SF-02150 Espoo, Finland)

The structural intensity methods offer a way of determining the absolute flow of mechanical power anywhere on the surfaces of some basic structures. In one-dimensional cases such as beams, these methods can be extended to the determination of mechanical impedance and reflection coefficient. In this paper, a separation of the bending vibration of the beam is suggested, which divides the field quantities into perfectly propagating and standing parts, based on the estimation of the complex reflection coefficient. Such a division is hoped to open an alternative view in quantifying the flow and distribution of power in beams. The technique is restricted to farfield regions of the beam. It is also directly applicable in other one-dimensional (vibrational, acoustical, etc.) fields. Properties of the suggested separation are illustrated with an experiment, and they are compared to an alternative approach that is based on the impedance. The relationship of the separated parts to the active and reactive intensity is discussed. It is shown that each of the field quantities involved in power transmission can be separated, and the propagating parts correspond to the net (active) intensity. The standing parts show properties that may be usable in modal analysis sense.

\section{1:35}

HH13. Effect of backlash and sleeve bearing clearance on the vibration spectrum of high-speed spur gear boxes. Fawzi M. A. El-Saeidy and T. Youshimura (Mechanical Engineering Department, Tokushima University, Minamijosanjima 2-1, Tokushima 770, Japan)

An analytical model is presented to simulate the effects of tooth backlash and sleeve bearing clearance on the vibration spectra of high-speed spur gear boxes. Elasticity in bending and torsion of the shafts, timevarying mesh stiffness, tooth backlash, and Hertzian mesh damping, friction between engaged teeth, and interaction between gear box casing and internals are accounted for. During contact between journal and bearing, where time-varying contact stiffness with consideration of damping at contact point is accounted for, bearing clearance, system vibration, and bearing forces are calculated. The analysis is applied to a single stage gear box and equations of motion are numerically integrated to obtain system response in the time domain. The response is transformed into frequency dornain using a fast Fourier transform (FFT) algorithm and samples of the results are shown for different values of backlash and bearing clearance. 
stiffness (stiffness is calculated based on the strain energies of the tooth, which is treated as a cantilever beam of involute shape), and Hertzian

HH14. On the vibration spectrum of high-speed spur gear boxes. Fawzi M. A. El-Saeidy (Mechanical Engineering Department, Tokushima University, Minamijosanjima 2-1, Tokushima 770, Japan )

An analytical model is presented to simulate the effect of tooth backlash and ball-bearing radial clearance on the vibration spectrum of highspeed spur gear boxes. Included in this model are elasticity in bending and torsion of the shafts, friction between meshing teeth, interaction between gear box casing and internals, and time-varying tooth backlash, mesh mesh damping. The bearing loads are calculated with consideration of shaft oscillations and speed, bearing radial clearance, ball centrifugal force, and bearing outer ring oscillations. To demonstrate the model, a single stage gear box is analyzed, and equations of motion are numerically integrated to obtain system response in the time domain. This response is transformed into the frequency domain using a fast Fourier transform (FFT) algorithm and samples of the results are shown for different values of backlash and bearing clearance.

\title{
Session II. Engineering Acoustics IV, Psychological Acoustics VII, and Physiological Acoustics III: Hugh S. Knowles Memorial Session
}

\author{
Mahlon D. Burkhard, Chairman \\ 1016 Raven Lane, Palatine, Illinois 60067 \\ Chairman's Introduction-9:00
}

Invited Papers

I11. Design evolution of miniature electroacoustic transducers. David P. Egolf, Elmer V. Carlson, Peter L. Madaffari, and August F. Mostardo (Industrial Research Products, Inc., Elk Grove Village, IL 60007)

This presentation traces the evolution of the design of miniature electroacoustic transducers during the last 40 years. Of particular emphasis are those contributions to the research, design, and development of such transducers made by Hugh Knowles and his colleagues. Pioneering efforts in the miniaturization of receivers (i.e., small loudspeakers or earphones) and microphones made possible the transition of hearing aids from the bulky body-worn aids of the past to the tiny all-in-the-canal aids of today. Included in this presentation is an overview of his innovations in piezoelectric, condenser (including electret) and electromagnetic transduction technology. Mr. Knowles' last contribution was the research and development of the miniature transducer-a hearing-aid receiver-incorporating a special electronic circuit to reduce current consumption and extend battery life of a hearing aid.

II2. Trends in hearing aids: From a technical to an audiological device. Poul Erik Lyregaard (Oticon Research Unit, Eriksholm, 243 Kongevejen, 3070 Snekkersten, Denmark)

In essence, a hearing aid is an acoustical amplifier, and has, therefore, traditionally been described in technical terms, as defined in the appropriate standards (IEC, ANSI). The advent of prescriptive fitting methods, plus a better understanding of the in situ acoustics, has had a significant impact on hearing aid design, but also on the way in which the acoustical properties are presented. Hearing aid dispensers tend to have an audiological rather than acoustical background, and the data to be employed in individual hearing aid fitting are also audiologically based. Hence, there is a strong case for manufacturers to adopt an audiological characterization of their hearing aids, even encompassing that hearing aid adjustments be calibrated in hearing loss and UCL units. Conversions between technical and audiological data for a hearing aid will be discussed and illustrated. In part, such conversions are contingent upon hearing aid measurements performed on the KEMAR manikin, one of Hugh Knowles' many contributions in the field of hearing aids. 
II3. Addressing Hugh Knowles' fundamental question: What would "high fidelity" mean for a hearing aid wearer? Mead C. Killion (Etymotic Research, 61 Martin Lane, Elk Grove Village, IL 60007)

First assigned by Hugh Knowles to update Hugh's earlier estimate of the difference between real-ear and $2-\mathrm{cm}^{3}$ coupler frequency-response curves for hearing aids, the author later did his $\mathrm{Ph}$.D. research on the topic of the design and evaluation of high-fidelity hearing aids. Throughout this research, Hugh's probing questions were both useful and sometimes embarrassing. He made it impossible to give superficial answers to difficult questions about (1) the occlusion-effect ("hollow voice") problem with closed-mold fittings, (2) what gain and frequency response would be required for a perception of high-fidelity sound by someone with a substantial high-frequency hearing loss, and (3) what directional/frequency-response accuracy in the hearing aid would be required for a "high fidelity" sense of the auditory space. Partly, as a result of Hugh's questions and contributions, a good solution has now been obtained to the occlusion-effect problem (reinventing Zwislocki's 1953 solution that he thought probably impractical), there are now transducers and "acoustic plumbing" techniques permitting $40-\mathrm{Hz}$ to $16-\mathrm{kHz}$ reproduction in hearing aids with fidelity comparable to that of professional studio monitors, in-the-canal hearing aids with directional properties comparable to that of the unaided ear, and a new integrated-circuit hearing-aid amplifier chip with level-dependent high-frequency emphasis that is designed to provide nearly "transparent" amplification to the wearer. Recorded demonstrations will be played if time permits.

\section{0:30}

II4. Electroacoustic test methods for evaluation of hearing-aid performance. L. B. Beck (Veterans Administration, Medical Center, 50 Irving Street, N.W., Washington, DC 20422), E. D. Burnett (National Institute for Standards and Technology, Gaithersburg, MD 20899), and G. D. Causey (Catholic University of America, Washington, DC 20064)

The purpose of this paper is to describe the development of electroacoustic test procedures for evaluation of hearing aids by the Veterans Administration (VA). These methods have evolved from the use of the $2-\mathrm{cm}^{3}$ coupler with pure tones as the input stimuli to the use of the KEMAR manikin with various types of complex noise as input stimuli. Hugh Knowles served as a consultant to the VA and his efforts, both for the VA and the research and development community at large, formed the framework for the evaluation program in place today. His contribution and leadership have resulted in procedures that permit the evaluation of a hearing aid in a manner very similar to the way it will be used by the wearer. Current use of FFT analysis and strategies for evaluating noise reduction circuits and in-the-ear hearing aids will be highlighted.

\section{0:55}

115. The acoustics of the external ear: Old problems and fresh perspectives. E. A. G. Shaw (Division of Physics, National Research Council Canada, Ottawa, Ontario KlA 0R6, Canada)

A coherent picture of the external ear, operating both in the free field and as an enclosed receiver, came into focus more than a decade ago though several troublesome problems remained, particularly at frequencies greater than a few $\mathrm{kHz}$. These included uncertainty about the geometry and acoustical characteristics of the ear canal, uncertainty about the dynamics of the eardrum and the reflection of sound from the eardrum, uncertainty about the role of the external ear in sound localization and, above all, uncertainty about the acoustical interactions between earphones and ears. Considerable progress has been made in most of these areas during the past few years but some problems remain intractable. In the meantime, the scientific and technological advances of the 1970s and early 1980 s have provided us with a legacy of valuable instruments: eardrum simulators, artificial heads especially the Knowles Electronic Manikin (KEMAR), microphones for probing ear canal pressure, and improved insert earphones.

\section{Contributed Papers}

\section{1:20}

II6. Evaluation of a real-time adaptive-beamforming hearing aid. Julie E. Greenberg and Patrick M. Zurek (MIT Research Laboratory of Electronics, Room 36-761, Cambridge, MA 02139)

A real-time two-microphone monaural hearing aid, based on the constrained adaptive beamformer proposed by Griffiths and Jim [IEEE Trans. Antennas Propag. AP-30, $27-34$ (1982) ], has been implemented and evaluated. The beamformer adapts to preserve the target signal (assumed to be straight ahead) and to minimize the power of jammer signals arriving from all other directions. The basic Griffiths-Jim algorithm is augmented with a method to inhibit adaptation in the presence of strong target signals [Greenberg et al., J. Acoust. Soc. Am. Suppl. 1 85, \$26 (1989) ]. The real-time system employs the Motorola DSP56001 and was evaluated using a speech target and a single jammer for a variety of freespace and head-mounted microphone configurations in anechoic and re- 
verberant environments. The system demonstrates very good performance (30- to 40-dB gain from input to output in an intelligibility-weighted spectral average of target-to-jammer ratio) for several broadside microphone configurations in the anechoic environment. Adaptation inhibition provides a robust insensitivity to target misalignment at high-input target-to-jammer ratios. As expected, performance degrades with increasing reverberation; at the critical distance, the system provides 5 - to $10-\mathrm{dB}$ gain. In extreme reverberation, the system performs at least as well as a delay-and-sum beamformer. [Work supported by NIH.]

\section{1:35}

II7. Optimization of hearing-aid gain and frequency response for cochlear hearing losses. Arne Leijon (Department of Information Theory, Chalmers University of Technology, S-412 96 Gothenburg, Sweden )
A new theoretical procedure was developed for optimizing the acoustic characteristics of hearing aids. The algorithm increases predicted speech intelligibility while keeping the estimated loudness of amplified speech at a predetermined level. The intelligibility criterion was the mutual information between spoken phonetic messages and the corresponding streams of auditory patterns in a psychoacoustic model of the listener's auditory system. The speech signal was modeled as a sequence of allophone transitions. Each transition was represented by three auditory patterns, each containing ten cepstrum coefficients, one voice and one duration parameter. The shape of optimized hearing-aid frequency responses depended strongly on the desired loudness of amplified speech. When normal loudness was desired for speech at $65 \mathrm{~dB}$ SPL, and the optimization was initialized with the NAL prescription, hearing-aid gain was reduced, but predicted speech intelligibility was nearly unchanged. Predicted intelligibility of amplified speech in a noisy background was similar for several different frequency responses. The mutual information was highly correlated with a modified version of the articulation index.

THURSDAY MORNING, 30 NOVEMBER 1989

ST. LOUIS BALLROOM H, 9:00 TO 11:45 A.M.

\author{
Session JJ. Underwater Acoustics V: The Sea Surface-Noise and Scattering \\ Eric I. Thorsos, Chairman \\ Applied Physics Laboratory, University of Washington, Seattle, Washington 98195
}

Chairman's Introduction-9:00

Contributed Papers

9:05

JJ1. Further studies of the underwater noise produced by rainfall. Paul A. Elmore, Hugh C. Pumphrey, and Lawrence A. Crum (National Center for Physical Acoustics, University of Mississippi, University, MS 38677)

A study of the sound produced by water drops striking a water surface has confirmed some earlier results [Pumphrey et al., J. Acoust. Soc. Am. 85,1518 (1989) ]. In particular, for a certain well-defined range of drop sizes and impact velocities, drops will predictably and repeatedly entrain bubbles; this phenomenon has been named regular entrainment. In the present study, various fixed drop diameters have been used to investigate how bubble frequency, dipole strength, and time between dropimpact and bubble formation vary with impact velocity. It is found that as impact velocity is increased through the point where entrainment begins, both frequency and dipole strength decrease to a minimum value and then rise again as the highest velocity at which entrainment occurs is approached. Both terms show increased variability near the critical upper and lower velocities. The frequency tends to increase monotonically as drop size is reduced; drops that entrain bubbles at their terminal velocities tend to produce frequencies near $14 \mathrm{kHz}$, which is also the peak frequency of the natural rainfall spectrum. Finally, the time between drop impact and bubble formation was found to increase monotonically as drop size or impact velocity increases. [Work supported by Office of Naval Research.]

\section{$9: 20$}

JJ2. An experimental investigation of bubble clouds as sources of ambient noise. S. W. Yoon, ${ }^{\text {a) }}$ L. A. Crum (National Center for Physical Acoustics, University of Mississippi, University, MS 38677), and A. Prosperetti (Department of Mechanical Engineering, The Johns Hopkins University, Baltimore, MD 21218)
Collective motions of bubble clouds are experimentally investigated as an ocean ambient noise source. The experiments, performed in a water tank, show that the frequency due to the bubble column resonance depends inversely on the logarithm of the void fraction of the water/bubble mixture. It is also observed that the frequency depends inversely on the volume of the bubble column. These experimental results indicate that the volume resonance of the bubble column is one of the more likely mechanisms for an ocean ambient noise source between 100 and $1000 \mathrm{~Hz}$. [Work supported by ONR and KOSEF (SWY).] " On leave from the Department of Physics, Sung Kyun Kwan University, Republic of Korea.

9:35

JJ3. Underwater sound generated by impacts and bubbles from raindrops at oblique incidence. Armagan Kurgan, ${ }^{\text {,) }}$ Herman Medwin, and Jeffrey A. Nystuen (Departments of Physics and Oceanography, Naval Postgraduate School, Monterey, CA 93943)

The sound generated by rainfall at sea is caused by raindrops of a wide size range that fall at their terminal velocities and generally strike the ocean surface at local, oblique incidence. Laboratory experiments have been conducted to evaluate the energy spectrum of sounds caused by both the impact and the bubble formed by single raindrops. The results, using terminal velocities and oblique trajectories, are very different from the published normal incidence, nonterminal-velocity characterizations. For example, bubble frequencies other than the well-known $14-\mathrm{kHz}$ peak are found. Also, the energy of the impact sound increases significantly for larger drops and for large deviations from normal trajectories. These observations provide specific reasons for the known broadening of the 14$\mathrm{kHz}$ spectral peak of rain noise in the presence of winds at sea. [Work supported by ONR. ${ }^{\text {a) }}$ Lt., Turkish Navy. 
JJ4. Acoustical measurements of spectral rates of bubble production under spilling breakers. Albert C. Daniel') and Herman Medwin (Physics Department, Naval Postgraduate School, Monterey, CA 93943)

It has been shown that spilling breakers in the laboratory produce a variety of bubbles whose total incoherent intensity is the Knudsen sea noise spectrum from 1 to $20 \mathrm{kHz}$ [H. Medwin and M. M. Beaky, J. Acoust. Soc. Am. 86, 1124-1130 (1989) ]. By using a hydrophone to simultaneously trigger both a "computerscope" and a spectrum analyzer, it is now possible to positively identify near-surface bubbles and to count their rate of production per unit area of the breaking wave. This passive acoustical determination spans a much greater range of bubble radii, with a radius resolution that is an order of magnitude finer than optical measurements [e.g., Y. Toba, Mem. Coll. Sci., Univ. Kyoto, Ser. A, XXIX(3) (1961) ]. [Work supported by ONR.] ${ }^{\text {a) }}$ Lt., U.S. Navy.
JJ5. Vertical directionality of low-frequency wind-induced noise. $B$. J. Sotirin and W. S. Hodgkiss (P-004, Marine Physical Laboratory of the Scripps Institution of Oceanography, UCSD, La Jolla, CA 92093)

The fine-scale structure of the directional ambient noise field is revealed using a large aperture $(900 \mathrm{~m})$ vertical array. Frequency and spatial spectral estimates are calculated during the passage of a local storm, providing a detailed study of ambient noise levels at low frequencies (15$130 \mathrm{~Hz}$ ) as wind speed increases from 2 to $12 \mathrm{~m} / \mathrm{s}$ over a 21 -h period. Spectral levels of beams directed horizontally show the time and spatial variability of distant sources: Spectral levels of beams directed toward the surface reflect local noise sources and display a threshold type behavior with level changes up to $5 \mathrm{~dB}$, suggesting the abrupt onset of a source mechanism such as breaking waves. Subsequent thresholds may indicate a change in source mechanism such as the conversion from spilling breakers to plunging breakers. [Work supported by ONT.]

\section{0:30}

JJ6. The scattering of sound by a cylindrical bubble cloud. Murray S. Korman (Physics Department, U. S. Naval Academy, Annapolis, MD 21402), Ronald A. Roy, and Lawrence A. Crum (National Center for Physical Acoustics, University of Mississippi, University, MS 38677)

Preliminary experiments are preformed on the scattering of acoustic pulses by a cylindrical bubble cloud. The goal is to investigate the role played by experimental length scales in establishing the angular dependence of the scattered intensity. These length scales include bubble diameter, interbubble distance, acoustic wavelength, and cloud diameter. The bubbles (which are larger than resonance size) are much smaller than the wavelength in water, which, in turn, is smaller than the diameter of the cloud. Experiments are carried out in a $1-\mathrm{m}^{3}$ tank using $250-\mathrm{kHz}$ acoustic pulses generated by a 5 -cm-diam circular plane array. An identical receiving array detected the scattered energy as a function of angle. The cylindrical bubble cloud is generated by a constant volume velocity airflow through a fritted ceramic disk located at the bottom of the tank. Results are compared with theories involving spherical clouds as well as homogeneous fluid cylinders with an "effective" compressibility and density. [Work supported by ONR.]

\section{1:00}

JJ8. Simulation of underwater acoustic scattering from large bubble plumes. Stanley A. Chin-Bing (Naval Oceanographic and Atmospheric Research Laboratory, Stennis Space Center, MS 39529) and Joseph E. Murphy (Department of Physics, University of New Orleans, New Orleans, LA 70148)

The finite-element ocean acoustic computer models, FFRAME and PE-FFRAME, have been used to gain insight into the dominant mechanisms involved in underwater acoustic scattering by large entrained bubble plumes near the ocean surface. Various types of incident acoustic fields (normal mode, PE, etc.) are used to interact with the plume while using the finite-element models to calculate the full-wave solution for the total field. Initially, the plume is treated as an acoustically soft boundary and various stages in the plume's life cycle are simulated. The simulation studies include the interaction of different propagating modes with the plume, and the effects of frequency and angle of incidence on the backscattered field.

\section{1:15}

JJ9. Acoustic scattering from a "Pierson-Moskowitz" sea surface. Eric I. Thorsos (Applied Physics Laboratory, University of Washington, Seattle, WA 98105)

An exact integral equation method has been used to study low-frequency $(200 \mathrm{~Hz})$ acoustic coherent and incoherent scattering from surfaces that vary in only one horizontal direction. The surface roughness spectrum is obtained from the Pierson-Moskowitz frequency spectrum for a fully developed sea. Comparisons are made with standard theoretical predictions using the Kirchhoff approximation, perturbation theory, and the composite-roughness model. The Kirchhoff approximation is found to be reasonably accurate for near-specular scattering and for the coherent reflection loss at grazing angles as low as $10^{\circ}$. Perturbation theory, when carried to order $(k h)^{4}$ for the scattering cross section, is in essentially perfect agreement with exact results for bistatic backscattering. This is true for $k h$ values as great as 1.8 , where $k$ is the acoustic wavenumber and $h$ is the rms waveheight. The standard composite-roughness model is found to be accurate for backscattering, except at the lowest grazing angles, where it over-predicts the scattering. [Work supported by ONR.] 
11:30

JJ10. Enhanced propagation in a foamy medium. A. Cowley (Admiralty Research Establishment, Portland, Dorset DT5 2JS, United Kingdom), C. H. Harrison (YARD, Scientific House, 40-44 Coombe Road, New Malden KT3 4QF, United Kingdom), A. Baird (Admiralty Research Establishment, Portland, Dorset DT5 2JS, United Kindgom), T. V. Gedrich, and C. A. Ferrari (YARD, Scientific House, 40-44 Coombe Road, New Malden KT3 4QF, United Kingdom)

Entrained bubbles have well-known effects on the acoustic properties of water, namely the velocity of sound, absorption, and backscattering. These, in turn, have a strong influence on sound propagation and rever- beration in the vicinity of a ship. A numerical model is discussed that uses as input data bubble population densities derived from hydrodynamic and bubble transport calculations, on a three-dimensional rectangular grid. Acoustic properties as a function of frequency are computed at each grid point using classical formulas, accounting for both resonant and nonresonant contributions. Relatively fast algorithms have been developed to calculate the three-dimensional ray paths (curved or straight rays), and transmission loss contours for arbitarily positioned source and receiver. Reverberation level as a function of time is determined assuming straight rays, but including simple multipaths and surface backscattering. Some interesting examples are presented including cases in which transmission may be enhanced by ducting or focusing at certain frequencies.

\author{
Meeting of Accredited Standards Committee S12 on Noise
}

to be held jointly with the

\title{
Technical Advisory Group for ISO/TC 43/SC1 Noise and ISO/TC 94/SC12 Hearing Protectors
}

\author{
W. Melnick, Chairman S12 \\ Ohio State University, University Hospital Clinic, 456 West 10th Avenue, Columbus, Ohio 43210
}

H. E. von Gierke, Chairman, Technical Advisory Group for ISO/TC 43/SC1

1325 Meadow Lane, Yellow Springs, Ohio 45387

Standard Committee S12 on Noise. Working group chairs will report on their progress under the plan for the production of noise standards. The interaction with ISO/TC 43/SC1 and ISO/TC $94 / \mathrm{SC} 12$ activities will also be discussed, with reference to the international standards under preparation. The Chair of the respective U.S. Technical Advisory Groups (H. E. von Gierke) will report on current activities of these committees. Plans for the next meeting of ISO/TC43 and ISO/TC43/SCl, to take place in Copenhagen during April 1990, will be discussed. 


\title{
Session KK. Engineering Acoustics V: Noise Sources, Analysis, and Active Cancellation
}

\author{
Howard H. Schloemer, Chairman \\ Naval Underwater Systems Center, New London, Connecticut 06320
}

Chairman's Introduction-1:00

Contributed Papers

KK1. Simultaneous screech tone modes of an axially symmetric jet. Y. Umeda, R. Ishii (Department of Aeronautical Engineering, Kyoto University, Kyoto 606, Japan), and Alan Powell (Department of Mechanical Engineering, University of Houston, Houston, TX 772044792)

The screech frequency of axially symmetric choked jets falls steadily with decreasing pressure ratio, with several frequency jumps, some up, some down (A. Powell, Proc. Phys. Soc. London Ser. B 66, 1039-1056 (1953) ]. Davies and Oldfield's Lissajous figures [Acustica 12, 267-277 (1962) ] showed that the corresponding jet oscillation modes are of various types. Schlieren photography and digitally processed acoustic pressure signals are now used. Unambiguously, the first two modes, $a_{1}$ and $a_{2}$, are axially symmetric (varicose, like ring vortices); the next, $b$, is asymmetric in an arbitrary plane (sinuous); $c$ is helical, rotating in either sense, stable once started, while $d$ is sinuous again, connected to $c$ by hysterectic jumps. However, tone $d$ is weakly but smoothly connected to $b$, so $d$ appears to be an extension of $b$. Moreover, the dominant $c$ tone extends weakly to overlap where $b$ and $d$ dominate, and a suspected weak $a_{2}(?)$ overlaps where $b, c$, and $d$ dominate. Further, both $a_{1}$ and $b$ exist weakly where $a_{2}$ dominates. Evidently jumps occur not necessarily at changes in the mode as previously thought, but when there is a change in which one dominates.

KK2. Recent experiments on impinging choked jet tones. Alan Powell and Brenda Henderson (Department of Mechanical Engineering, University of Houston, Houston, TX 77204-4792)

Two modes of oscillation, $L$ and $S$, associated with large and small plates were described, with frequency analysis by counter [A. Powell, J. Acoust. Soc. Am. 83, 515-533 (1986) ]. With different slopes on $\log -\log$ plots of wavelength versus nozzle-to-plate spacing $h$, feedback to nozzle and to stand-off shockwave, respectively, was hypothesized. Digital signal processing is more revealing! The same dominant and $S$ tone are found, exactly. It now appears these $L$ and $S$ are the dominant members of classes of tones. At small $h$, for large plates (all pressures) and small plates (low pressures), $L$ tones are observed in multiple modes, usually simultaneously. For intermediate $h$, frequency jumps occur; schlieren photography reveals symmetric and asymmetric jet flow oscillations. Nozzle reflector influence supports the feedback to the nozzle hypothesis for $L$. For large $h$ at low pressures, three strong constant tones exist-screech $a_{1}$ and $a_{2}-$ and one other(?). At higher pressures (small plates), $S$ tones appear, infiuenced by a nozzle reflector (!); schlieren photographs exhibit symmetrical oscillations of jet and stand-off shockwave. For intermediate plates, both $L$ and $S$ classes occur.
KK3. The sources of trans-spectral coherence in the radiation from rotating blades. P. G. Vaidya (Department of Mechanical and Materials Engineering, Washington State University, Pullman, WA 99164), Jeffrey J. Girard (The Aerospace Corporation, Mail Station M4-910, El Segundo, CA 90245), and Carey L. Moulten (Wyle Research, El Segundo, CA 90245)

Rotating blades (installed in fans, propellers, and helicopters) radiate both discrete and broadband sound. This radiation can be examined for the coherence of the trans-phase. Trans-phase is the phase difference between the components at two different frequencies when one of them has been sent through a nonlinear filter. If the trans-phase shows coherence, the signals are said to be trans-spectrally coherent. Various sources of the rotating machinery noises contribute to the coherence in different ways. Existence of such coherence can help in the diagnostics and identification of the sources and in the detection and classification of the machinery in presence of extraneous noise.

\section{1:50}

KK4. On the feasibility of the evaluation of the performance of a pinger transducer in an open tank using a pressurized vessel. V. N. Bindal, $T$. K. Saksena, S. C. Gupta, and D. R. Chaubey (National Physical Laboratory, New Delhi-12, India)

Investigation on the calibration of pinger transducers to a hydrostatic pressure of $200 \mathrm{psi}$ is described. The transducer is housed in a specially constructed vessel fabricated from fiberglass in polyester and epoxy matrix of $10-\mathrm{mm}$ wall thickness. The insertion loss of the walls of the vessels has been estimated in the frequency range $10-20 \mathrm{kHz}$ and is observed to be small. Source level measurements have been made by putting the pressurized vessel in a tank of dimension $6.35 \mathrm{~m} \times 5.85 \mathrm{~m} \times 2.2 \mathrm{~m}^{3}$. The merits and demerits of the vessels using an epoxy and polyester base have been discussed. Measurements show that there is no significant variation in acoustic output of the pinger transducer up to $200 \mathrm{psi}$, although a variation is observed in the frequency response characteristic. The method is suitable for characterization of a transducer at high pressures that cannot be characterized by using conventional techniques.

\section{2:05}

KK5. Accurate spectral estimation of multiple sinusoids using an FFT. John C. Burgess (Department of Mechanical Engineering, University of Hawaii, Honolulu, HI 98622)

Accurate estimates of amplitude, frequency, and phase of multiple sinusoids in a signal can be obtained using a method described earlier [ $\mathbf{J}$. C. Burgess, J. Acoust. Soc. Am. Suppl. 1 83, S92 (1988) ]. FFT size required for specified spectral resolution, as well as some computational aspects, is discussed. 
KK6. Low peak factor waveforms for efficient system analysis using a fast Fourier transform. John C. Burgess (Department of Mechanical Engineering, University of Hawaii, 2540 Dole Street, Honolulu, HI 96822)

Low peak factor waveforms [M. R. Schroeder, Number Theory in Science and Communication (Springer, New York, 1984)] can be designed so that multiple harmonics in the waveform appear to have the ideal spectrum of a true Fourier transform when the waveform is analyzed using a fast Fourier transform (FFT). If the harmonics in the waveform are restricted to those having an integral number of cycles in the FFT data record length, there is no leakage, and the magnitudes and phases of the Fourier coefficients are identical to those of the harmonics in the waveform. Low peak factor waveforms drastically reduce peak power requirements. For example, the peak power requirement for a waveform having 63 harmonics can be reduced by a factor of about 30 . Waveforms can be designed having harmonics with constant, octave, and close approximations to one-third octave frequency differences. This approach can provide an efficient alternative to other methods in current use for measuring system transfer functions. [Work supported in part by the National Research Council of Canada and the Lanikai Foundation.]

\subsection{5}

KK7. Application of the fast Hartley transform to industrial noise analysis. Woon S. Gan (Acoustical Services Pte Ltd., 29 Telok Ayer Street, Singapore 0104, Republic of Singapore)

The application of the fast Hartley transform (FHT) to industrial noise analysis to replace the fast Fourier transform (FFT) is proposed. The Hartley transform of a real-valued function is also real-valued. Its evaluation does not involve complex functions and it has the same formula for forward and inverse transform. Thus it has a speed advantage over FFT. The type of FHT algorithm chosen is due to H. V. Sorensen et al. [IEEE Trans. Acoust. Speech Signal Process. ASSP-33, 1231-1238 (1985) ]. As an illustration, the two-channel FFT signal processor is chosen. The computation of the following functions is repeated: autocorrelation function, cross-correlation function, power spectrum, and the acoustic intensity of real-numbered data by replacing the FFT with FHT. A detailed analysis of the time that can be saved in computation by using FHT is made. A key advantage of the Hartley transform technique for large autocorrelation is the use of the same formula for transforming and inverse transforming.
KK9. Sound-pressure distribution on a cross section of a duct for active noise cancellation. Kang Yen, Osama A. Mohammed, Vijay Raman, and Kurt S. Ramdin (Department of Electrical Engineering, Florida International University, Miami, FL 33199)

Active cancellation techniques for low-frequency noise have been applied to some industrial plants. Field tests show very useful results. In the past, research work has concentrated on the theory of minimization of the enclosed sound field along one axis. However, in practice the direction of the sound source is uncertain. Especially when the noise source is very close to the duct, the direction of the sound wave will be a dominant design factor in order to achieve the cancellation at the other end of the duct. In this paper, a set of directional and finite-size cancellation sources inside the duct is considered. The sound-pressure distribution across any cross section in the duct is the primary research interest. The results provide information on the design of noise cancellation devices such as noise cancellation earphones. [Work supported by the Florida High Technology and Industry Council.]

KK10. Active attenuation of pure tones in a lined duct using adaptive filtering. Dominique Laget and Elmer L. Hixson (Department of Electrikal and Computer Engineering, University of Texas, Austin, TX 78712)

Fan noise is characterized by strong discrete blade passing tones added to broadband aerodynamic noise. In air conditioner ducts, absorptive liners provide reduction of the noise at the higher frequencies but are ineffective at lower frequencies. An active adaptive controller is mostly effective for low-frequency narrow-band noises. In this study, noise control combining a duct liner and an adaptive attenuator has been implemented. The stability and convergence properties of the adaptive attenuator depend on the compensation of the transducers and acoustic delays. Compensations using an IIR filter, a delay, and an FIR filter designed with the LMS algorithm have been studied. After simulation, the controllers based on a filtered- $x$ and a delayed-x LMS algorithm have been implemented on a digital signal processing board. Measurements inside and outside a lined air conditioner duct showed significant pure-tone attenuation, especially when a novel filtering-x method is used

KK8. Finite element simulation of an active noise-canceling scheme. Osama A. Mohammed, Kurt S. Ramdin, Vijay Raman, and Kang Yen (Department of Electrical Engineering, Florida International University, Miami, FL 33199)

This paper details a finite-element computer simulation scheme of an axis-symmetric physical setup of an active noise canceling headset. The results of this simulation show the distribution of sound-pressure levels inside the ear canal. Two-dimensional simulations of an axis-symmetric configuration of the ear cup were carried out. A point source was used to cancel out the noise. From these simulations it can be concluded that the noise inside the ear canal can be reduced. The amount of reduction depends on the strength and phase of the canceling source. On the other hand, a point source is not a practical device. To overcome this problem, new structures for speakers have to be created and simulated to check if their radiation is in accordance with that of practical devices. An algorithm proposed earlier by these authors for the estimation of the SPL noise reduction that can be accomplished inside the ear canal has been added. [Work supported by the Florida High Technology and Industry Council.]
KK11. Numerical simulation of a sound field generated by flow around a circular cylinder. Z. C. Zhu and L. Z. Zeng (Department of Engineering Mechanics, Tsinghua University, Beijing, People's Republic of China)

Flow around a circular cylinder is a classical problem, but it is difficult to simulate a Karman vortex street in the wake numerically. In previous numerical works artificial rotation had to be added to the cylinder to separate the vortex from it. Obviously it would influence the original solution. In addition, body conditions were usually not satisfied correctly in many numerical works. There are two major improvements in this paper. (1) In order to separate the vortex, it is proposed that only one asymmetrical grid-point condition is given at the farfield of the wake. (2) A recent treatment is used to guarantee that body conditions are satisfied precisely. Then numerical simulation of flow around a circular cylinder can be completed correctly and successfully, with the application of ADIBGE schemes to solve incompressible N-S equations in the form of stream function and vorticity at Reynolds numbers from 1 to 1000 . Sound radiation is calculated by numerical integrating $\mathrm{FW}-\mathrm{H}$ equations at an $\mathrm{Re}$ of 100 . Then careful analysis and comparison are made for all parts of sound sources. Finally several useful conclusions are given. 
stable. It is also time-saving and storage-saving. Maybe it is a good method

KK12. Numerical analysis of sound propagation with acoustic shock waves in varying area ducts. $Z$. C. Zhu and $Y$. Zhang (Department of Engineering Mechanics, Tsinghua University, Beijing, People's Republic of China)

In recent results, several second-order finite difference schemes were adapted to obtain shocked solutions for sound propagation in varying area hard-wall ducts with transonic flow [N. J. Walkington and W. Eversman, J. Sound Vib. 104, 81-107 (1986)]. The second-order MacCormack scheme with a fourth viscous term was then recommended by the authors. But its numerical error still seemed to be a bit large. In this paper, the fourth-order MacCormack scheme with a fourth viscous term is used to improve the solution. It is shown from three sample calculations with acoustic shock waves that the new method is much better than the secondorder MacCormack method. Its numerical error, on an average, is only one fifth of that in the latter method. Here the new method is depicted in detail, considering especially the treatment of boundary conditions, the selection of viscous coefficient, the stability condition, and the computation correctness criterion. This method needs less grid points and is more for calculating shocked solutions of sound propagation in ducts.

4:05

KK13. The physics model of thyristor interference in radios and other communication equipment. Ming-Fu Yang, Fei Xue, and Yi-Pei Lu (No. 357 Xun Yang New Village, Shi Quan Road, Shanghai 200061, People's Republic of China)

With the increasing use of thyristors in electric installations such as lamp controllers, electric fan controllers, and electric heat controllers, thyristor interference in the middle frequency $(f<20 \mathrm{MHz})$ range is repugnant and seriously hampers the use of thyristors. A physical model of thyristors indicates that interference results from the instantaneous pulse that is produced when the thyristor is turned on. Such interference will produce an interference pulse on a nearby antenna that is received and amplified to become annoying noise. This conclusion is useful in the design of electric installations using thyristors so that such installations have greater reliability and better interference prevention.

\title{
Session LL. Physical Acoustics VI: Ultrasound and Nondestructive Evaluation
}

\author{
Allan D. Pierce, Chairman \\ Graduate Program in Acoustics, Pennsylvania State University, State College, Pennsylvania 16804
}

Contributed Papers

\section{1:00}

LL1. Elastic constant reconstruction using resonances of coupled modes in thin anisotropic plates. S. I. Rokhlin, C. Y. Wu, and L. Wang (Department of Welding Engineering, The Ohio State University, 190 West 19th Avenue, Columbus, $\mathrm{OH} 43210$ )

At oblique incidence of ultrasonic waves on a thin anisotropic composite plate immersed in fluid, the quasilongitudinal, flexural, and quasi$\mathrm{SH}$ vibrations are excited. At some angles of incidence, coherent interactions of these waves lead to minima of the normal displacement on the back surface of the plate and therefore to minima of the transmission coefficient. At off-axis orientations of the thin composite plate, two such minima may be observed. Their positions are only slightly dependent on frequency and are determined by plate properties and orientation. This effect was studied both theoretically and experimentally. The elastic constants have been reconstructed for a unidirectional graphite-epoxy composite plate from experimental data that have been taken at different plate orientations and frequencies.

\section{1:15}

LL2. On the transition between slip and rigid boundary conditions between two solid media. Y. J. Wang and S. I. Rokhlin (Department of Welding Engineering, The Ohio State University, 190 West 19th Avenue, Columbus, $\mathrm{OH} 43210$ )

In order to describe the transition between slip and rigid boundary conditions, a model with a thin interphasial viscoelastic layer is introduced. By changing the relaxation time $(\omega \tau)$, the transition from a vis- cous liquid to a solid layer is observed. When the interface layer is in the liquid state with very small shear nodulus the shear stress components on the boundary vanish, resulting in a slip boundary condition. On the other hand, after the layer is solidified, the two semispaces become welded with the transition of shear and longitudinal stresses to one another. It is shown that the ratio of a hydrodynamic boundary layer thickness to the interface layer thickness plays a major role in this transition. A simple approximate equation for the reflection coefficient from the interface is derived and compared to the exact solutions for different transitional stages of the boundary condition. Theoretical predictions are compared to experimental results.

\section{$1: 30$}

LL3. Method for measurement of elastic constants of thin anisotropic membranes. W. Wang and S. I. Rokhlin (Department of Welding Engineering, The Ohio State University, 190 West 19th Avenue, Columbus, $\mathrm{OH} 43210$ )

In this work the elastic properties of a thin layer of porous aluminum oxide obtained by phosphorus anodization have been studied by a novel ultrasonic technique. The thickness of the porous oxide samples is about $50 \mu \mathrm{m}$ with through vertical pores of about $2000 \AA$ diameter and porosity of about $70 \%$. The ultrasonic technique is based on measurements of the angle of the minimum of the transmitted energy at oblique incidence on the immersed sample. The effective elastic constants that have been theoretically calculated from the experimental data are in good agreement with that found from estimations of bulk properties of anisotropic porous media. The knowledge of the properties of porous anisotropic anodized 
$\mathrm{Al}_{2} \mathrm{O}_{3}$ layers has major importance for adhesive joint characterization since such layers play an important part in interphasial strength of the joint.

\section{$1: 45$}

LL4. Focusing of ultrasound in solids with applications to QNDE. John G. Harris (Center for Quality Engineering and Failure Prevention, Catalysis Building, 2137 North Sheridan Road, Evanston, IL 602083020)

Ultrasound can be focused by the curved fluid-solid interface of a solid part being interrogated for cracks or by using a lens as part of an imaging system such as the acoustic microscope. In the latter case it is usually difficult to form a diffraction-limited spot when the focus lies in the solid because the focused beam field must cross a fluid-solid interface. This paper summarizes recent investigations of these two situations. A calculation of the wave fields near the caustics formed when a two-dimensional (unfocused) beam scatters from a concave fluid-solid interface is discussed. Also discussed is a calculation of the wave fields within the focal region of a three-dimensional, wide aperture source when the focused beam is directed across a plane fluid-solid interface and the focal region lies in the solid. In both cases attention is paid to the interplay between the diffraction effects of the source and of the focal region, and also to the excitation of leaky surface waves. The implications of these considerations for the quantitative interpretation of ultrasonic measurements are explored.

\section{2:00}

LL5. Guided wave generation by direct excitation. Peter B. Nagy and Laszlo Adler (Department of Welding Engineering, The Ohio State University, Columbus, $\mathrm{OH} 43210$ )

Guided acoustic waves along interfaces and more complex layered structures are often used in ultrasonic NDE. Their outstanding sensitivity to specific properties associated with interface conditions and bond quality is due to the fact that their energy is effectively confined to the region of interest. On the other hand, this advantage turns out to be a great drawback for generation and detection of such guided waves. Certain modes with higher phase velocity than at least one of the bulk velocities of the surrounding media are called leaky modes that can be coupled to via phase matching bulk waves. True guided modes of lower phase velocity than any of the bulk velocities of the surrounding media are much more difficult to generate and detect. A simple experimental technique based on longitudinal or shear wave excitation along the interface is shown to be very effective in generating both symmetric and antisymmetric guided modes of Rayleigh (SV) or Love (SH) type of polarization. Experimental results from different plates and adhesive and solid-state interfaces are shown to be in good quantitative agreement with theoretical calculations. This technique might find numerous applications in basic acoustic studies as well as ultrasonic nondestructive evaluation. [This work was supported by the Office of Naval Research under Contract N0014-C-2129.]

\section{2:15}

LL6. Radiated fields determined from moments of surface velocity. David D. Bennink and Anna L. Pate (Department of Engineering Science and Mechanics, Iowa State University, Ames, IA 50011)

A quick and efficient method is derived for calculating the field radiated by a finite but otherwise arbitrary velocity profile. The method calculates the pressure field radiated into the half-space $(z>0)$ from the normal component of velocity on the boundary plane $(z=0)$. The derivation starts from the Rayleigh integral and utilizes a power series expansion of the kernel in the radial coordinate of the source point. As a result, a formulation is obtained that contains separate terms for the source and for propagation. The source terms represent the moments of the velocity profile. The propagation terms can be obtained from a recurrence relation starting from certain special cases for which analytical expressions are known. Convergence and stability of the method will be discussed, and examples of field calculations for a few simple profiles will be given. [Work supported by Center for NDE at Iowa State University.]
LL7. Surface motion reconstruction based on half-space field data. David D. Bennink and Anna L. Pate (Department of Engineering Science and Mechanics, Iowa State University, Ames, IA 50011)

A finite but otherwise arbitrary normal velocity profile at the boundary of a half-space is expended in a set of orthonormal functions. The radiation problem is then formulated in terms of the moments of this profile. (Details of this formulation are given in the preceding abstract.) The radiation problem is discretized to obtain a matrix formulation and a singular value decomposition method is used to estimate the velocity profile from measurements of the radiated field. Additional constraints are imposed upon the derivatives of the profile in order to overcome the illposed nature of the inverse source problem. The accuracy of the method will be discussed in terms of both the positioning of the field locations (nearfield versus farfield) and the weighting of constraints. Examples of velocity profile reconstruction will be given for a few simple radiators. [ Work supported by Center for NDE at Iowa State University.]

\section{2:45}

LL8. A prior knowledge based optimal Wiener filtering approach to ultrasonic scattering amplitude estimation. Steven P. Neal (Mechanical and Aerospace Engineering, University of Missouri-Columbia, Columbia, MO 65211) and Donald O. Thompson (Center for NDE, Iowa State University, Ames, IA 50011)

In order to interpret measurements of the scattered wave field resulting from sonification of an object, the effects of the measurement system must be removed from the measured data. In ultrasonic nondestructive evaluation, estimation of the farfield scattering amplitude for a flaw in a material, e.g., a crack, void, or inclusion, involves removing the measurement system effects in the presence of electronic noise and noise due to the scattering of sound from inherent material features such as grain boundaries. The purpose of the work reported here was to evaluate an optimal Wiener filtering approach to scattering amplitude estimation. The filter was shown to determine an optimal estimate as the weighted average of the information derived from measurement of the scattered wave field and prior information about the flaw distribution. The optimal Wiener filter was evaluated and compared to a nonoptimal form of the Wiener filter. The optimal Wiener filter was shown to provide improved scattering amplitude estimates by filtering out the noise and by utilizing prior flaw information. [Work supported by the Director of Energy Research, Office of Basic Energy Sciences. ]

\section{3:00}

LL9. The measurement and analysis of acoustic noise as a random variable in ultrasonic nondestructive evaluation. Steven $P$. Neal (Department of Mechanical and Aerospace Engineering, University of Missouri-Columbia, Columbia, MO 65211) and Donald O. Thompson (Center for NDE, Iowa State University, Ames, IA 50011)

Sound scattered from inherent material features such as grain boundaries becomes a source of acoustic noise when attempting to detect or characterize a flaw in a material, e.g., a crack, void, or inclusion. In formulating a stochastic model to describe a flaw detection or flaw characterization experiment, acoustic noise is often assumed to be an uncorrelated, Gaussian random variable with zero mean and known average power spectrum. The work reported here focuses on the measurement of acoustic noise and on the evaluation of these assumptions for the cases of scattering from grains in stainless steel and scattering from porosity in aluminum, respectively. An estimate of the average power spectrum is determined for each case. It is shown that in both the time domain and in the frequency domain the acoustic noise considered has zero mean and is reasonably uncorrelated and Gaussian. [ Work supported by the Director of Energy Research, Office of Basic Energy Sciences.] 


\title{
Session MM. Psychological Acoustics VIII and Physiological Acoustics IV: General Physiological and Psycho- logical Acoustics (Poster Session)
}

\author{
Robert H. Gilkey, Chairman \\ Central Institute for the Deaf, 818 South Euclid Avenue, St. Louis, Missouri 63110
}

All posters will be displayed from 1:00 to 3:50 p.m. To allow all contributors an opportunity to see other posters, contributors of odd-numbered papers will be at their posters from 1:00 to 2:25 p.m. and contributors of even-numbered papers will be at their posters from 2:25 to 3:50 p.m.

\section{Contributed Papers}

MM1. Behavioral thresholds for tone pips in 3- and 6-month-old infants and adults. Lynne Werner Olsho, M. Patrick Feeney, and Richard C. Folsom (Departments of Otolaryngology and Speech and Hearing Sciences, University of Washington, WJ-10, Seattle, WA 98195)

Infant behavioral thresholds for tone-pip stimuli at 1,4 , and $8 \mathrm{kHz}$ (3cycle rise-fall and 1-cycle plateau) were estimated using the observerbased psychoacoustic procedure (OPP). Behavioral thresholds for these stimuli were also obtained on a group of normal-hearing adults. Across frequency comparisons revealed that 6-month-old infant threshold curves paralleled adult curves, but infant thresholds were elevated 20 to $30 \mathrm{~dB}$. For 3-month-old infants, OPP threshold curves were not parallel to adult curves due to relatively poorer infant thresholds at $8 \mathrm{kHz}$. Furthermore, in comparison with wave $\mathrm{V}$ auditory brain-stem response ( $\mathrm{ABR}$ ) thresholds for the same stimuli, 3-month-old infant behavioral thresholds are the same as ABR thresholds at 1 and $4 \mathrm{kHz}$, whereas behavioral thresholds are poorer at $8 \mathrm{kHz}$. A similar elevation in threshold at $8 \mathrm{kHz}$ for 3 month olds has been reported for longer duration stimuli [ $L$. W. Olsho et al., J. Acoust. Soc. Am. 84, 1316-1324 (1988)]. The results suggest a frequency-dependent maturation of behavioral thresholds between 3 and 6 months for these brief stimuli. [Work supported by NIH.]

MM2. Central processing in patients with cochlear implants. D. M. Daly (Box 210855, Dallas, TX 75211), F. D. Owens, and P. Stephenson (3600 Gaston Avenue, Suite 1103, Dallas, TX 75246)

Because of locations and orientations of auditory cortex, studies of central processing in man typically exploit psychophysical phenomena or disease altered structure/functioning. Cochlear implants afford a view of central processing through sparse, specifiable peripheral transfer functions. Six patients were examined who have unilateral implants (cochlear 22 channel; 14-2l active electrodes in $\mathrm{BP}+1 ; 4 \mathrm{AS}, 2 \mathrm{AD}$ implants; 2 100 weeks post-op) using prerecorded sets of BW, GY, BDG, and ite delivered directly through the processor [Daly et al., J Neurophysiol. 140 (2), 141-62 (1980) ]. The representation of stimuli was sufficient for three patients to classify stop/glide sets consistently $(\rho<0.001)$; although in a set which included both BW and GY none distinguished [be] from [ge] or [we] from [ye]. Performance reverted to chance when the processor was configured as a single channel device and all stimuli had the same total duration. None distinguished among BDG. One patient classified vowels ile consistently; boundaries changed significantly when a central electrode malfunctioned and was inactivated in configuration map. Results suggest, with these stimuli, patients resolved brief temporal changes more accurately than brief frequency changes alone.

MM3. Psychometric functions for level discrimination in simulated and cochlear impairment. Søren Buus (Communication and Digital Signal Processing Center, 409 DA, Northeastern University, Boston, MA 02115), Mary Florentine, and Tilmann Zwicker (Communication Research Laboratory, 133 FR, Northeastern University, Boston, MA 02115)
To determine whether the form of psychometric functions for $2 \mathrm{I}$, 2AFC level discrimination changes with hearing impairment, measurements were obtained in listeners with cochlear impairments and normal listeners with impairments simulated by masking. Ten increment levels were presented in random order within blocks of 100 trials. Stimuli were tones at $0.25,1,8$, or $14 \mathrm{kHz}$ or white noises chosen to encompass a wide range of conditions and difference limens. Durations were either 10 or 500 $\mathrm{ms}$ and levels ranged from 50 to $90 \mathrm{~dB}$ SPL. For each condition, at least 20 blocks were presented in mixed order. Results show that the sensitivity $d^{\prime}$ is nearly proportional to $\Delta L(=20 \log [(p+\Delta p) / p]$, where $p$ is pressure) over the entire range of difference limens for both simulated and real impairments as well as for normal listeners tested in the quiet. These results indicate that if the transformation from stimulus intensity to decision variable is affected by hearing impairment or masking, the change affects both the mean and the standard deviation in the same manner. They also lend support to the notion that $\Delta L$-plotted on a logarithmic scale-is an appropriate representation of level-discrimination performance. [Work supported by NIH.]

MM4. Electrotactile encoding and recognition of a 16-obstruent system. H. G. Piroth, S. Gfroeter, and H. G. Tillmann (Institut für Phonetik und Sprachliche Kommunikation, Schellinstrasse 3, 8000 Munich 40, Federal Republic of Germany)

In continuation of a long-term training experiment $[H$. G. Tillmann and H. G. Piroth, J. Acoust. Soc. Am. Suppl. 1 84, S45 (1988)] the recognition of 16 obstruents in Cə syllables was tested using a modified quasiarticulatory coding method. Tactile syllable patterns were delivered by the 16-channel electrocutaneous system "SEHR-2" to 16 electrode pairs that were arranged to build four equidistant rings around the left forearm. Consonants were assigned to rings or pairs of rings in order to yield more than four places of articulation. Fricatives were coded as subsequent stimulations of four or eight electrodes (forming one or two rings, respectively) while plosives were represented by oscillating movements between the neighboring electrodes of a ring. The fortis/lenis distinction was coded by different pattern velocities. Since recognition with the initial coding of the two-ring fricative patterns as parallel and synchronous movements was poor, an alternative coding method was applied with contrarotating movements in order to avoid local masking or inhibition resulting from the stimulation of adjacent electrodes. Using this alternative coding method, one highly trained subject reached $85.6 \%$ correct identifications of the 16 obstruents.

MM5. Consonant testing of a tactile hearing aid based on amplitude- and time-quantized speech. Edward M. O'Brien (Bioengineering Program, Texas A\&M University, College Station, TX 77843-3120)

Speech was first prefiltered with a second-order bandpass filter centered at $1.5 \mathrm{kHz}$. Speech was then amplitude quantized to two levels based on the zero crossings of the waveform. This signal was then time quan- 
tized such that it could only change states in association with a clock signal. The intelligibility of the processed speech remains high for clock frequencies above $10 \mathrm{kHz}$ (which was the quantizing rate used for this study). The discrete digital frequencies in the resulting waveform were easily detected with a digital logic circuit. These were then displayed onto the skin of the abdomen with a linear array of concentric ring electrodes. Thirty-one electrodes (representing frequencies between 151 and 2500 $\mathrm{Hz}$ ) were used for the array. Biphasic constant current pulses were used as the stimuli. Five normal hearing college age students served as the subjects. Discrimination of consonant pairs was used to test the ability of the tactile aid to transmit speech information to the subjects. The following ranges of accuracy (corrected for guessing) were observed: stops and constituents, $18 \%$ to $87 \%$; voiced and unvoiced consonants, $3 \%$ to $27 \%$; consonants with different places of articulation, $27 \%$ to $90 \%$; nasal, glides, and semivowels, $17 \%$ to $49 \%$.

MM6. Noise reduction hearing aids: A tutorial. Ruth A. Bentler and Brenda B. Bergman (Department of Speech Pathology and Audiology, University of Iowa, Iowa City, IA 52242)

In the last few years, there has been a proliferation of (so-called) noise reduction hearing aids onto the market. While some of these designs are little more than multiband compresson circuits, others employ adaptive filtering (dependent upon overall input level), adaptive compression (dependent upon duration of high-level input signals), and variations thereof. The purpose of this poster session is twofold: (1) using graphics and schematics an explanation of six available "noise reduction" circuits will be provided. This will serve as a tutorial for those audiologists attempting to keep up with design and marketing strategies for noise reduction; (2) preliminary results of a large clinical project begun late in 1988 aimed at determining how effective different circuits are for different configurations and/or degrees of hearing loss in new and experienced users will be presented. Success of effectiveness will eventually be measured over a 12-month period using (a) speech recognition (in quiet and noise), (b) portions of the Hearing Performance Inventory (Giolas and Owens, 1979), (c) expectations checklist (Seyfried and Anderson, 1989), (d) qualitative measure task (modified from Gabrielson and Lindstrom, 1985), as well as (e) ratings of "satisfaction" taken at 6 months and 12 months post fitting.

MM7. Regression effects in magnitude judgments of line length, and vibrotactile sine and pulse loudness. Amy A. Collins and Roger W. Cholewiak (Department of Psychology, Green Hall, Princeton University, Princeton, NJ 08544-1010)

Five observers performed absolute magnitude estimation (AME) and absolute magnitude production (AMP) of line lengths and vibrotactile pulse and sine loudnesses as well as matching of each possible pair. Line lengths, presented visually, could extend over a 7 - to $950-\mathrm{mm}$ range. Vibrotactile stimuli were 32-pps haversine pulses and $250-\mathrm{Hz}$ sinusoids presented to the palm with 7-mm diameter contactors and static surrounds. When the AME and AMP data were plotted together, perfect correlation was not seen indicating the influence of regression effects on the data. Regression effects occur when observers making judgments on two perceptual continua (i.e., line magnitude and number magnitude) restrict the range of their responses gravitating toward the mean response. Magnitude balance functions (MB) were calculated from AME and AMP data for the line, sine, and pulse. The cross-modality matches, made with each stimulus type serving as the standard, showed similar regression effects. These data are discussed with reference to transitivity and the relationships among the data set predicted from the absolute scaling hypothesis. [Work supported by NIH.]
MM8. Evidence for a tight blood-labyrinth barrier. Naoki Inamura, Alec N. Salt, and Ruediger Thalmann (Department of Otolaryngology, Washington University, St. Louis, MO 63110)

The entry of a small tracer ion (FW 135.6) from blood into perilymph and cerebrospinal fluid (CSF) has been monitored using ion-selective electrodes. The tracer, trimethylphenylammonium (TMPA) can be detected in extremely low concentrations $(<1 \mu \mathrm{m})$ by electrodes, allowing entry to be accurately quantified. Electrodes were sealed into the cochlea to ensure that artifactual fuid leakages did not distort the results. A constant level of TMPA in plasma (approximately $0.5 \mathrm{mM}$ ) was maintained by continuous venous infusion of tracer. TMPA entry into CSF was extremely slow, reaching only $13 \%$ of the plasma level within $90 \mathrm{~min}$ $(n=3)$. Tracer entered perilymph even more slowly, reaching $6.5 \%$ $(n=5)$ and $3.7 \%(n=5)$ of the plasma level in $90 \mathrm{~min}$ for ST and SV, respectively. The slow entry of such a small ion into perilymph demonstrates the existence of a tight barrier between blood and perilymph, and eliminates the possibility that perilymph is generated by mechanisms such as ultrafiltration. In two animals where bleeding was noted at the electrode insertion site, a much more rapid rate of tracer entry was recorded, probably as a result of plasma leakage into perilymph. Treatment with epinephrine (giving a brief blood pressure increase) also opens the barrier, allowing rapid tracer entry.

MM9. Action potential tuning curves recorded with a derived response technique. Philip Garcia and Alec N. Salt (Department of Otolaryngology, Washington University, St. Louis, MO 63110)

A number of laboratories use tonal masking methods in conjunction with the cochlear action potential (AP) to generate AP tuning curves. In these studies, the amplitude of AP elicited by a probe stimulus is monitored as the level of masker is varied. An amplitude reduction (AR) by a specified amount $(25 \%-100 \%)$ is used to indicate masking of the response. An alternative method was investigated in which interaction of the probe and masker is monitored by digital subtraction of the unmasked and masked responses, generating a tone-derived (TD) response. An amplitude criterion of $10-\mu \mathrm{V}$ TD response is used to indicate masker/probe interaction. Tuning curves were recorded from the round window of anesthetized guinea pigs using AR and TD methods. With near threshold probe stimuli, similar tuning curves were obtained. With higher levels however, markedly different results were observed. AR tuning curves became elevated and sharper while TD curves became lower and broader. The TD method appears to better represent the frequency spread of responses with increasing stimulus level as demonstrated at the single unit level. In addition, TD tuning curves were able to detect second harmonic distortions of the probe stimulus, while AR tuning curves did not. [Work supported by Program project grant P01 NS24372 and Training Grant NS 07278.]

MM10. Coding of phase structure in complex sounds by bullfrog auditory-nerve fibers. Andrea Megela Simmons and Michael Ferragamo (Department of Psychology, Brown University, Providence, RI 02912)

This experiment explored how the peripheral coding of the spectral and temporal structure of a complex communication signal is affected by the phase relations between individual frequency components in the sound. Complex sounds with 21 components, all of which were harmonics of a common, low-frequency fundamental $(100 \mathrm{~Hz})$, were digitally synthesized. The frequencies and amplitudes of the individual components matched those of the bullfrog's species-specific advertisement call. For one stimulus, the 21 components were generated in cosine phase, and for the other stimulus, the 21 components were in random phase. Most amphibian papilla (AP) and basilar papilla (BP) fibers synchronized to the envelope only of the phase-coherent stimulus, and the complex spectral structure of the stimulus was not accurately encoded by population firing patterns. Envelope synchronization to the random-phase stimulus was not seen in the responses of some mid-frequency-sensitive AP and BP fibers, although low-frequency-sensitive AP fibers responded similarly to both stimuli. Behavioral experiments suggest that bullfrogs can discriminate between in-phase and random-phase versions of their advertisement calls. 
MM11. Post-discharge recovery effects in gerbil auditory-nerve period and PST histograms. Walter R. Bosch (Institute for Biomedical Computing, Washington University, St. Louis, MO 63110)

Excitation-function (EF) and recovery-function (RF) parameters of a self-exciting point process (SEPP) model for auditory nerve discharges were estimated [M. I. Miller, J. Acoust. Soc. Am. 77, 1452-1464 (1985)] for neural discharges recorded in gerbil in response to continuous-tone and tone-burst stimuli. The mean discharge intensity (MDI) of the SEPP model (expected value of the PST or period histogram) was computed from the EF and RF estimates by solving an integral equation [ $\mathrm{K}$. Jones $e t$ al., J. Acoust. Soc. Am. 78, 90-94 (1985) ]. Period histograms computed from neural discharges in response to continuous-tone stimuli were found to agree almost exactly with the corresponding MDI over a range of stimulus sound-pressure levels from 0 to $50 \mathrm{~dB}$ above threshold, indicating that the SEPP model accounts well for post-discharge recovery effects in period histograms under these stimulus conditions. PST histograms computed from the neural response to tone-burst stimulation also agreed quite well with the computed MDI for the conditions tested. [Work supported by NIH grants NS21592 and RR01379.]

MM12. Neurophysiological observations on the auditory cortex in the FM bat, Eptesicus fuscus. Cynthia F. Moss (Department of Psychology, Harvard University, Cambridge, MA 02138), Jonathan Fritz, Michael Ferragamo, and James A. Simmons (Department of Psychology and Section of Neurobiology, Brown University, Providence, RI 02912)

The echolocating FM bat, Eptesicus fuscus, integrates target range and shape (range profile) into unified images along a synthetic targetrange axis. The response properties contained in single- and multi-unit recordings from the auditory cortex of Eptesicus in relation to the process of image formation revealed in psychophysical data were examined. A tonotopic map was found in primary auditory cortex similar to what has been described previously, but also a much larger auditory cortical representation than hitherto reported. Echo delay-tuned neurons are interspersed with frequency-tuned neurons, showing no apparent topographic organization. The response profiles of some echo-delay-tuned and delaytracking neurons are dependent on the harmonic structure of the FM stimuli, as required for representing the unusual dual time- and frequency-domain images that the bat perceives. Strong suppression effects may sharpen perceived target range and be the basis for encoding the depth structure of sonar targets in Eptesicus.

MM13. ABER latencies are prolonged in rat pups fed a biotin deficient diet. L. P. Rybak, C. Whitworth, V. Scott, and B. Bhardwaj (Department of Surgery, SIU School of Medicine, Springfield, IL 627949230)

The effects of biotin deprivation on ABER were studied in rat pups fed a diet that lacked this compound. Pregnant rats were either fed a normal rat diet (controls) or a special diet without biotin (Teklad, Harlan Sprague Dawley, Madison, WI). After weaning, the respective diets were continued in pups from each group. ABER was tested under chloral hydrate anesthesia $(380 \mathrm{mg} / \mathrm{kg})$ using clicks $(100 \mu \mathrm{s}, 5 / \mathrm{s})$ and tone bursts (3-ms duration, 1.5-ms rise-fall time at $2,8,16$, and $32 \mathrm{kHz}$ ) presented free field with a driver suspended $8 \mathrm{~cm}$ above the vertex in the median plane. Responses were obtained using an active lead from the vertex with a reference electrode in the nose. Blood levels of biotin were measured using a microbiological assay. Animals on the biotin-free diet showed alopecia and dry, scaly skin. There was no significant difference in ABER threshold in control versus experimental animals. However, there was a signifcantly longer brain-stem transmission time (interpeak latencies from waves I to IV) in biotin-deficient animals. The lowering of serum biotin in experimentals was confirmed by microbiologic assay. These findings suggest that biotin deficiency in the developing rat adversely affects auditorynerve and/or brain-stem development. These effects could be mediated by a delayed myelination of these structures. [Work supported by Deafness Research Foundation.]
MM14. Auditory-nerve discharge characteristics that distinguish between basilar papilla neurons and amphibian papilla neurons in the leopard frog, Rana pipiens. Don A. Ronken (Institute for Biomedical Computing, Washington University, St. Louis, MO 63110)

The frog's inner ear has two sensory organs for auditory reception. One organ, the amphibian papilla (ap), has many of the complexities of mammalian ears. The other organ, the basilar papilla (bp), is much simpler and offers special opportunities for comparison to the mammalian inner hair cell configuration. These anatomical differences make it important to identify the site of the receptor cell when recording from individual afferent neurons in the main trunk of the auditory nerve. In routine experiments, this can be a difficult problem, since the characteristic frequency (cf) of the highest-frequency (ap) neurons overlaps the cf of bp neurons in many species. To approach this problem, a statistical data base was created using responses to tone bursts and clicks, tuning curve characteristics, and spontaneous rates. A standard statistical method, discriminant analysis, produced reliable classification of neurons without using cf estimates and offers the possibility of a fully automated decision procedure. [Work supported by NIH grants NS21592 and RR01379.]

MM15. Cochlear mechanical model stability. John W. Matthews and Charles E. Molnar (Institute for Biomedical Computing, Washington University, St. Louis, MO 63110)

The introduction of nonlinear damping into computational simulations of stable active cochlear mechanical models has introduced instability in computational simulations of models used here. Nonlinear dynamical systems theory provides tools that give insight into the possible sources of such instability. Analysis of differential equations for a simplified cochlear mechanical model with a computer program ("AUTO" by Eusebius Doedel) suggests that the unstable behavior of earlier simulations is an artifact of the computations rather than an inherent feature of the differential equation model. [Work supported by NIH grants NS21592 and RR01379.]

MM16. Perceptual fusion of tonal and noisy sounds. Dik J. Hermes (Institute for Perception Research/IPO, P.O. Box 513, NL 5600 MB Eindhoven, The Netherlands)

If stationary noise is added to a tonal, periodic sound, the noise is, to a large extent, perceived as a separate sound coming from a source other than the periodic sound. Perceptual fusion of noise with a periodic sound requires specific conditions. Results will be presented that indicate that the temporal envelope of the noise plays an important role in this matter. In experiments in which this envelope had the same peridocity as the tonal stimulus, perceptual fusion did occur. Furthermore, the energy content of the noise within successive periods of the envelope was made more or less constant. These results were obtained in an experimental setup in which a quantitative measure was obtained for the extent to which the noise integrated with the periodic sound. It will be argued that perceptual fusion as reported here cannot be explained by a peripheral mechanism such as adaptation, but must be the result of a central process which groups components from a wide range of frequency bands into one or more sound images.

MM17. Vowel formant frequencies produced with and without auditory feedback. Emily A. Tobey and Christy Murchison (Department of Communication Disorders, Louisiana State University Medical Center, New Orleans, LA 70112)

Several authors have hypothesized that auditory feedback is used to refine articulatory gestures in children developing speech. In order to examine the role of auditory feedback in children, vowel production was examined in 12 children ranging in age from 3-13 years who received a multichannel cochlear implant. Data were collected 1 year after using the implant. Speech samples consisted of ten repetitions of the word, "head," spoken under four conditions: implant on, immediately after the implant 
was turned off, 20 min after nonuse, and immediately after the implant was turned on. Formant frequencies, fundamental frequencies, and duration were measured. Significant shifts in vowel formant frequencies were found when the implant was turned off $(p<0.0001)$ and returned to more nearly normal values when the implant was turned on. Two of the youngest subjects were unable to produce stimuli after 20 min of nonuse with the implant. Data support the hypothesis that auditory feedback may assist in refining the articulatory gestures of some speech sounds.

MM18. Extracting stable measures from unstable psychometric performance. Marjorie R. Leek (Army Audiology and Speech Center, Walter Reed AMC, Washington, DC 20307-500I), Thomas R. Hanna, and Lynne Marshall (NSMRL, Box 900, SUBASE NLON, Groton, CT 06349)

At the last meeting of the Acoustical Society of America, a new method of estimating slopes of psychometric functions when thresholds were varying over the course of an experiment was reported. Computer simulations were used to calculate the relationship between the true slope of the function and the variability across pairs of trials in two interleaved adaptive tracks. Further, the variability across all trials in the two tracks was shown to indicate the stability of the function over time. Here, the validation of that procedure in the measurement of human performance is reported. Listeners were asked to detect a $1000-\mathrm{Hz}$ tone in a narrow band of noise, the level of which was either fixed or varied sinusoidally from trial to trial. This masker variation imposed systematic threshold shifts analogous to those in the simulations. The period of sinusoidal shift ranged from 16-128 trials per cycle, and the amplitude of shift was either 4 or 8 dB. Psychometric functions for individual listeners were constructed both by traditional methods of fitting the trial-by-trial data, and by using the functional relationship between slope and variability determined from the computer simulations. When the imposed threshold shift did not occur too rapidly, the newer method provided more stable slope estimates than did the traditional fitting methods. Under certain conditions the procedure was able to identify the unstable performance. These results show that this interleaved tracking technique can produce more stable slope estimates, and can alert experimenters to drifting in subject performance.

MM19. Prior auditory stimulation influences the precedence effect. Ruth Y. Litovsky, Richard L. Freyman, Uma Balakrishnan, and Rachel K. Clifton (University of Massachusetts, Amherst, MA 01003)

Previous studies have demonstrated that echo threshold for clicks is elevated by a preceding click train. The present study examined the influence of a preceding click train for three variables presented in 16 combinations: click rate ( $1-16 / \mathrm{s})$, number of clicks in the train (3-17), and duration of train (0.5-8.0 s). Subjects were tested in an anechoic chamber, with two speakers positioned at $45 \mathrm{deg}$ left and right of midline. Leading clicks were always presented from the left speaker, while lagging clicks were presented from the right speaker with delays ranging between 2-14 $\mathrm{ms}$. Each trial consisted of a click train followed by the test click. Subjects pressed a button when they heard the lagging test click on the right. Subjects' echo threshold was defined as the click delay at which the echo was reported on $50 \%$ of trials. Relative to an isolated test click, thresholds were elevated approximately 3 to $6 \mathrm{~ms}$ when preceded by a click train. The most critical variable influencing magnitude of shift in echo threshold was the number of clicks in the preceding train. For a constant number of clicks, duration of the train had little effect, whereas rate sometimes interacted with number. [Work supported by NSF.]

MM20. Is a power function description of intensity-jnd data compatible with the loudness function? William S. Hellman (Department of Physics, Boston University, Boston, MA 02215) and Rhona P. Hellman (Department of Psychology, Northeastern University, Boston, MA 02115)

A sequence of approximation for calculating loudness functions from the intensity-jnd function for a $1-\mathrm{kHz}$ tone is performed. It is shown that a single power function representation of pure tone intensity-jnd data can generate a loudness function from a McGill-Goldberg type model in better agreement with loudness measurements than has heretofore been demonstrated. This improvement arises from the presence of an integration constant that had been previously ignored. The inclusion of the integration constant markedly improves the overall shape of the derived loudness function, somewhat more at low then at high intensity levels. Using the same near miss power function, the consequences of setting the integration constant equal to zero are indicated. These effects are compared to those obtained from the unaltered intensity-jnd data [W. S. Hellman and R. P. Hellman, J. Acoust. Soc. Am. Suppl. 1 82, S25 (1987) ]. Corrections resulting from going beyond first order in the calculational procedure are discussed. [Partially supported by the Rehabilitation R\&D Service of the VA.]

MM21. Increased CMR resulting from lowered levels of masker band. Marion F. Cohen (Department of Communication Sciences, The University of Connecticut, Storrs, CT 06268)

Comodulation masking release (CMR) for a $1000-\mathrm{Hz}, 50-\mathrm{ms}$ sinusoidal signal was measured in the usual manner, except that the overall level of the 100 - $\mathrm{Hz}$-wide masking noise band was varied from 18 to $78 \mathrm{~dB} \mathrm{SPL}$ in 20-dB steps. The cue band was always $78 \mathrm{~dB}$ SPL. Presentation was either monotic or dichotic, with the cue band presented to the nonsignal ear only. Results show CMR values averaging as high as $7 \mathrm{~dB}$ when the cue band was centered at $8000 \mathrm{~Hz}$, three octaves away from the signal, and $12 \mathrm{~dB}$ when it was two octaves away. CMR was greatly reduced when the two noise bands were at equal levels. These results were essentially replicated dichotically, except that CMR was somewhat smaller. These data relate to previous results showing poorer detectability of a noise-band signal when a synchronous noise band is introduced at a distant frequency [M. F. Cohen and E. D. Schubert, J. Acoust. Soc. Am. 81, 721-723 (1987); D. McFadden, J. Acoust. Soc. Am. 81, 1519-1527 (1987)]. [Work supported by AFOSR.]

MM22. Homophasic and antiphasic masking as a function of signal bandwidth and frequency region. John $P$. Preece, David A. Eddins, and Richard H. Wilson (Audiology, VA Medical Center, Long Beach, CA 90822 and University of California, Irvine, CA 92664)

Thresholds for homophasic and antiphasic multicomponent tonal complexes were measured as a function of bandwidth in broadband noise to replicate and to extend the classic masking experiment [G. Gässler, Acoustica 4, 408-414 (1954) ]. Gässler reported that masked thresholds for tonal complexes were nearly constant for bandwidths less than a nominal "critical bandwidth," beyond which thresholds increased $3 \mathrm{~dB} /$ oct. Three experiments were conducted in broadband noise employing (a) homophasic signals similar to Gässler's ( 1 to 20 sinusoids spaced $20 \mathrm{~Hz}$ apart extending downward from $1100 \mathrm{~Hz}$ ); (b) homophasic and antiphasic signals composed of 1 to 23 sinusoids centered at 500,1000 , and 2000 $\mathrm{Hz}$; and (c) homophasic and antiphasic signals centered at $500 \mathrm{~Hz}$ with bandwidths to $800 \mathrm{~Hz}$ ( 71 sinusoids). The slope of the threshold/signal bandwidth function beyond $100 \mathrm{~Hz}$ was $<3 \mathrm{~dB} /$ oct at each center frequency, ranging from $0.3 \mathrm{~dB} /$ oct $(2000 \mathrm{~Hz})$ to $1.9 \mathrm{~dB} /$ oct $(1000 \mathrm{~Hz})$. The data indicate that the integration of signal energy continued beyond the so-called "critical band" estimates reported by Gässler. There were no substantial differences between the slopes of the homophasic and antiphasic conditions at 500,1000 , and $2000 \mathrm{~Hz}$. [Work supported by VA Medical Research Service.]

MM23. Psychometric functions for unattended tones. Huanping Dai, Soren Buus, and Bertram Scharf (Northeastern University, Boston, MA 02115)

Listeners detect attended tones better than unattended tones. To determine how much better, percentage correct detection was measured as a function of signal level for tones at 24 unattended frequencies, all more 
than a critical band from the attended or primary frequency of $1000 \mathrm{~Hz}$. The primary was presented as a cue at the beginning of every trial (2I, $2 A F C$ ) and also as the signal on $67 \%$ of the trials. On the rest of the trials, the signal could be any one of 24 different probe frequencies. All signals were presented against a wide-band noise at $62 \mathrm{~dB}$. Nearly 6000 trials by three experienced subjects yielded a mean psychometric function for the probes that was parallel to that for the primary, but shifted to the right, showing poorer detection of the probes. Closer analysis revealed that the 14 probes at frequencies above $1000 \mathrm{~Hz}$ were "attenuated" 2 to $3 \mathrm{~dB}$, and the 10 probes below were attenuated 4 to $6 \mathrm{~dB}$. These and earlier results suggest that probe frequencies may be effectively attenuated $6 \mathrm{~dB}$ or more. [Work supported by NIH.]

MM24. Functional involvement of auditory cortex in fine frequency discrimination of biosonar signals in the bat. $H$. Riquimaroux, $S$. J. Gaioni, and N. Suga (Department of Biology, Washington University, St. Louis, MO 63130)

The auditory cortex of the mustached bat, Pteronotus parnellii, has a highly specialized region, the DSCF (Doppler-shift constant frequency) area, which overrepresents the frequencies of Doppler-shifted echoes (about $61 \mathrm{kHz}$ ), and, hence, may play a role in the fine frequency discrimination necessary for detailed velocity measurements (Suga, 1984). This function of the DSCF area was behaviorally tested using reversible ablation with muscimol, a potent GABA agonist (Hilosaka et al., 1985). Bats were trained on a discriminated shock avoidance task requiring a leg flexion response. The stimuli were trains of artificial pulse-echo pairs (tone bursts). For $\mathbf{S}+$, the pulse and echo were the same frequency (e.g., $61.0 \mathrm{kHz}$ ), while, for $\mathrm{S}-$, they were different frequencies (e.g., 61.0 and $61.1 \mathrm{kHz})$. Following baseline testing, $0.1-0.2 \mu($ of muscimol $(1 \mu \mathrm{g} / \mu \ell$ saline) was bilaterally applied to the DSCF area. The bats failed on previously successful discriminations of small frequency differences (e.g., 50 $\mathrm{Hz}$ ), but succeeded on discriminations of large frequency differences (e.g., $2 \mathrm{kHz}$ ). Discrimination performance returned to baseline levels within $24 \mathrm{~h}$. These data indicate that the DSCF area is necessary for fine frequency discriminations involving biosonar signals. [Work supported by AFOSR Grant No. 87-0250.]

MM25. Evaluation of a physiologically based auditory model using psychophysical data. Patricia S. Jeng') (Center for Research in Speech and Hearing Sciences, City University of New York, New York, NY 10036) and Joseph L. Hall (Acoustics Research Department. AT\&T Bell Laboratories, Murray Hill, NJ 07974)
A physiologically based auditory model [O. Ghitza, J. Phon. 16(1), 109-123 (1988)] was tested against human psychophysical data. The model consists of a set of cochlear filters inferred from frequency selectivity of primary auditory fibers in cat, rate-level processing, and synchrony extraction. Isoloudness contours for pure tones are calculated from the model and are compared to those measured in human by Fletcher and Munson (1933) and by Robinson and Dadson (1956). The model is also used to simulate Scharf's [J. Acoust. Am. 31, 365-370 (1959)] criticalband measurements using complex sounds of four components. In addition, both calculated and measured critical-band results are plotted as a function of cochlear place using formulas for human and cat cochlear maps in Greenwood's recent paper (submitted to J. Acoust. Soc. Am.). The comparison of calculated and measured data suggests possible improvements of the model. " Work done as a consultant to AT\&T Bell Laboratories, Acoustics Research Department.

MM26. Effects of auditory segregation of a harmonic component on discrimination of diotic intensity and of interaural time difference. $W$. S. Woods and H. S. Colburn (Boston University, 48 Cummington Street, Boston, MA 02215)

Researchers have demonstrated that the just-noticeable difference (jnd) for interaural time difference (ITD) of a tonal target increases in the presence of tonal maskers, even with target/masker separations greater than a critical band [Buell and Hafter, J. Acoust. Soc. Am. Suppl. 1 83, S122 (1988); Stellmack et al., J. A coust. Soc. Am. Suppl. 1 85, S83 (1989) ]. In most cases, subjects report the perception of one "sound object," whose subjective position is affected by the ITD at each frequency of the stimulus. It has also been noted that asynchronizing the onset times of components of a stimulus yields more than one "sound object." This experiment investigates whether this parsing can lead to unmasking of a component, and whether a general model of parsing can be used to predict observed effects. A 4I, 2AFC adaptive procedure was used to measure diotic intensity and ITD discrimination jnd's for a $100-\mathrm{ms}, 600-\mathrm{Hz}$ target, both in isolation and when masked by a two-tone masker comprising 400 and $800-\mathrm{Hz}$ tones, under varying conditions of relative onset time of masker and target. Although generally lower jnd's were obtained for the asynchronous case, the fact that the difference was small relative to the variability in the adaptively measured jnd's prompted a fixed-increment measurement of sensitivity under the same conditions. [Work supported by NINCDS.]

\title{
Session NN. Speech Communication VIII: Speech Perception
}

\author{
David B. Pisoni, Chairman \\ Speech Research Laboratory, Department of Psychology, Indiana University, Bloomington, Indiana 47405
}

Contributed Papers

1:00

NN1. Converging evidence on the nature of the segmental representation underlying spoken word recognition. Deborah A. Gagnon and James R. Sawusch (Department of Psychology, SUNY at Buffalo, Buffalo, NY 14260)
Previous work has shown that the segmental representation used in recognizing spoken words does not correspond to the traditional abstract phoneme [Gagnon and Sawusch (1989)]. However, the evidence did not unequivocally distinguish between two alternative proposals-allophones and position-specific phonemes. In the previous study, subjects heard natural CVC prime-target pairs in various degrees of phonetic over- 
lap and named the second item (the target) as quickly as possible. The present study adopted the same approach but utilized a phoneme monitoring task in which subjects responded whenever the target began with a designated phoneme. The pattern of RT results, while different from that obtained in the naming task, again supported a rejection of the phoneme and shed further light on the nature of the representation. Similar patterns across word and nonword blocks and across trials in which voice was the same versus different within a prime-target pair were found with both tasks. A comparison will be made of the two task types in terms of the results generated and the implications for their use in studying on-line spoken word recognition. [ Work supported by NINCDS.]

1:12

NN2. The role of phonological permissibility in the phonetic coding of speech. Carol A. Wannemacher and James R. Sawusch (Department of Psychology, Park Hall, SUNY/Buffalo, Buffalo, NY 14260)

Previous research has shown that phonological permissibility of a consonant sequence can affect the identification of an ambiguous phoneme [Massaro and Cohen (1983)]. The present experiment used both reaction time and identification measures to further explore this finding. With natural speech, little or no effect of phonological permissibility of a fricative-liquid sequence was found upon speed to classify the liquid. In the second experiment, a synthetic liquid series was constructed with endpoints varying in permissibility (depending on the fricative precursor). A speeded classification task was run and data were partitioned according to speed of response (cf. Fox, 1984). The results of this experiment, combined with the earlier findings, illustrate the relative roles of both acoustic-phonetic and phonological information in phonetic coding. The data will be discussed in terms of the data- and knowledge-driven influences upon phonetic coding. [Work supported by NINCDS.]

$1: 24$

NN3. Attention to phonetic context across word boundaries. Jenny DeGroot and Howard C. Nusbaum (Department of Psychology, University of Chicago, 5848 S. University Avenue, Chicago, IL 60637)

Variability in phonetic context affects phoneme perception. For example, subjects are slower to identify a target consonant when an adjacent context phoneme varies independently, compared to when context phonemes are constant across trials [C. C. Wood and R. S. Day, Percept. Psychophys. 17, 346-350 (1975) ]. Further, this is true whether of not a syllable boundary intervenes between the target and the varying context, suggesting that subjects attend to phonetic context regardless of syllable structure [J. DeGroot and H. C. Nusbaum, J. Acoust. Soc. Am. Suppl. 1 $85, \mathrm{~S} 123$ (1989) ]. Do listeners similarly attend to phonetic context across a word boundary when recognizing a phoneme? In the present experiments, subjects identified a target phoneme, while an adjacent context phoneme either varied orthogonally or was held constant. In one stimulus set, the varying context occurred in the same word as the target; in the other stimulus set, the vrying context occurred in an adjacent word. Response times were measured to investigate whether the varying context slows target recognition across a word boundary as it does within a word. The results provide information about how listeners distribute their attention across the speech signal, and about the perceptual function of different linguistic units. [Research supported by NIH.]

\section{1:36}

NN4. The effects of attention on the phonetic integration of acoustic information. Jennifer $\mathbf{L}$. Eberhardt and Peter $\mathrm{C}$. Gordon (Department of Psychology, Harvard University, Cambridge, MA 01238)

The present study investigated the effects of attention on phonetic processing. Thirty-five synthetic steady-state vowels varying from $/ \mathrm{i} /$ to
/1/ were used. The first three formants of each vowel varied in seven equal logarithmic steps from $/ \mathrm{i} /$ to $/ \mathrm{I} /$ as the duration varied from short to long in five steps $(50,80,120,190$, and $300 \mathrm{~ms})$. All of the vowel stimuli were presented to subjects under high- and low-attention conditions. Attention was manipulated by requiring that subjects perform a nonspeech distractor task while simultaneously performing a speech identification task, or by requiring that subjects perform a speech identification task only. Phonetic identification of the vowel stimuli was found to vary with the attention condition. When subjects performed the distractor task and the speech task simultaneously, duration became a more important cue to phonetic identity, whereas the effect of formant frequency was reduced. A quantitative model was developed to characterize how the integration of information from the two kinds of cues, formant structure and duration, changed with attention level.

\section{$1: 48$}

NN5. The intelligibility of speech directed to children and adults. Judith C. Goodman, Howard C. Nusbaum, Lisa Lee, and Kevin Broihier (Committee on Cognition and Communication, University of Chicago, 5848 S. University Avenue, Chicago, IL 60637)

Speech directed to very young children differs markedly from speech directed to adults in vocabulary, syntax, and intonation. Are there differences in segmental intelligibility as well? Speech was recorded from six mothers addressing their 2 year olds, and, in another session, addressing an adult, under comparable conditions. Open and closed-class words were excised from both types of speech and were presented, in isolation, to adult listeners for identification. Children may not understand closedclass words, so mothers may not articulate them clearly. Excised openclass words expressed either new or previously given information. Whereas repeated information is articulated less clearly in speech to adults, repeated information in speech to children may be intended to aid comprehension. Thus the given/new distinction may affect the intelligibility of speech to adults and to children differently. Preliminary results indicate that child-directed speech may be less intelligible than adult-directed speech, particulary for closed-class words: Expectations about children's linguistic knowledge apparently affect articulation of different word classes. [Work supported by NIH and a Biomedical Research Support Grant. ]

\section{2:00}

NN6. Cues to perceptual normalization of talker differences. Todd M. Morin and Howard C. Nusbaum (Department of Psychology, The University of Chicago, 5848 S. University Avenue, Chicago, IL 60637)

To recognize vowels produced by a specific talker, a listener must know something about the talker's vowel space. How do listeners learn about a talkers' vowel space? How different must two talkers' vowels be to constitute different vowel spaces? To investigate these questions, listeners monitored sequences of target vowels for a specified target. In one condition, a single talker produced the vowels for each trial. In a second condition, a mix of four talkers produced the vowels for each trial. Previous research demonstrated that vowel recognition is accurate when talkers are mixed, but it requires more attention. The present study compared recognition for whispered and voiced vowels in these conditions to eliminate the use of $F 0$ as a cue for talker normalization. Another experiment examined the effects of talker differences on normalization. Performance for a pair of talkers with similar vowel spaces was compared to performance for a pair of talkers with more disparate vowels. The results of these studies indicate the importance of fundamental frequency in vowel recognition across talkers and that the amount of effort required for normalization of talker differences depends on the similarity of the different talkers' vowel spaces. [Work supported by NIH.]

\section{2:12}

NN7. On the percepturl representation of vowel categories. Keith Johnson (Speech Research Laboratory, Psychology Department, Indiana University, Bloomington, IN 47405) 
Subjects' behavior in a vowel perception task was compared with the predictions of two different types of vowel perception models. Both models use auditory spectra generated by a model of the peripheral auditory system [Bladon and Lindblom, J. Acoust. Soc. Am. 69, 1414-1422 (1981) ]. In the first model, it is assumed that the spectral information in vowels is represented as a whole spectrum. The spectral characteristics of a vowel category are modeled as a spectral template against which incoming vowel spectra are compared. In the second model, the spectral information in vowels is characterized by the frequency locations of spectral peaks. Here, the spectral component of a vowel category is taken to be information concerning the frequencies of spectral peaks. Two sources of vowel templates for the models were also compared. In one case, templates were derived from the subject's own productions of the vowels in question. In the other, templates were derived from vowels synthesized with average formant values reported by Peterson and Barney [J. Acoust. Soc. Am. 24, 175-184 (1952) ]. Subjects were instructed to find the best exemplars of each of 11 different vowel categories from an array synthetic steady-state vowel tokens by adjusting the $F 1$ and $F 2$ of the tokens until the best exemplar was located. In all cases, it was found that the whole spectrum approach provided the best fit to the subjects' perceptual judgments. It was also found that model predictions based on the Peterson and Barney vowels provided a better fit to the perceptual data than did predictions using the subject's own productions as the basis for spectral templates. This finding suggests that perceptual vowel categories are not subject to the idiosyncrasies of the individual's own speech production, but rather are a product of the listener's range of the perceptual experience.
NN8. The intelligibility of LPC-vocoded words and sentences presented to native and non-native speakers of English. Molly Mack (Division of English as an International Language, 3070 Foreign Languages Building, 707 S. Mathews, University of Illinois, Urbana, IL 61801 and the MIT Lincoln Laboratory, Lexington, MA 02173)

The present study is an extension of previous work [M. Mack, J. Neurolinguist. 3, 293-316 (1988); M. Mack., J. Acoust. Soc. Am. Suppl. 181, S4 (1987) ] designed to assess the intelligibility of natural and computer-generated speech presented to native and non-native speakers of English. In this study, subjects were presented with three tests-the Diagnostic Rhyme Test (DRT), a meaningful sentences test, and a semantically anomalous sentences test. Stimuli were presented in two conditionsnatural and LPC ( $2.4 \mathrm{kbps}$ ) vocoded. Subjects were 20 native speakers of English and 20 native speakers of German, fluent in English. Results revealed that the non-natives performed significantly worse than the natives on all but the natural-speech DRT; word frequency affected accuracy more for the non-natives than natives; and in the vocoded condition, the non-natives appeared to exhibit fatigue effects, unlike the natives, who exhibited perceptual learning. Moreover, the effects of test type and condition upon non-native response accuracy were nonadditive. Implications for models of non-native speech processing and the use of coded speech systems by non-native listeners are considered.
NN9. Comprehension of natural and synthetic speech. James V. Ralston, John W. Mullennix, Scott E. Lively, Beth G. Greene, and David B. Pisoni (Speech Research Laboratory, Department of Psychology, Indiana University, Bloomington, IN 47405)

Previous studies comparing the comprehension of natural and synthetic speech passages have produced conflicting results. All of these studies used successive measurement techniques in which subjects' comprehension was assessed after the presentation of a passage. However, comparative studies have found that successive methods are less sensitive than simultaneous "on-line" measures. Successive measures are also known to be influenced by memory factors. Subjects in the present experiment monitored for word targets while they listened to short passages and then verified statements after each passage. Both monitoring and verification performance for passages of synthetic speech were depressed relative to passages of natural speech. There was a significant interaction between voice and text difficulty in the monitoring latency data, suggesting that both factors affect mechanisms drawing from the same limited processing resources. Finally, there was a significant interaction between voice and sentence type in the verification task. Subjects listening to passages of synthetic speech had relatively poor memory for propositional information compared to surface (word) information. Taken together, the results indicate that comprehension of synthetic speech is poorer and slower than natural speech. Memory for propositional information extracted from passages of synthetic speech is particularly poor. [Work supported by NSF.]

2:48

NN10. Perception of Hindi retroflex versus dental stops by monolingual speakers of American English. Linda Polka (Speech Perception Laboratory, Department of Communication Sciences and Disorders, University of South Florida, Tampa, FL 33612)

Cross-language studies have shown that foreign consonant contrasts vary in the degree of perceptual difficulty that they present adult nonnative listeners. Phonemic, phonetic, and acoustic factors have been considered important in accounting for this variability. These factors were examined by comparing English listeners' perception of the Hindi retroflex versus dental place-of-articulation contrast in four different voicing contexts: prevoiced, voiceless aspirated, voiceless unaspirated, and breathy voiced. Differences in the perceptual difficulty of the four Hindi contrasts were predicted based on: (1) phonemic status (the functional status of the contrast in listeners' native phonology), (2) phonetic familiarity (as allophones or free variants), (3) differences in acoustic salience related to voicing, and (4) assimilation strategies. Differences in performance in a categorial $\mathrm{AX}$ discrimination task were ordered from most to least errors: prevoiced, voiceless aspirated, breathy-voiced, and voiceless unaspirated. Perceptual differences were correlated with both acoustic salience of place cues and subjects' descriptions of their assimilation strategies. [Work supported by NICHD and NINCDS.]
$3: 15$

NN11. Effects of auditory and phonetic training on Americans' discrimination of Hindi retroflex-dental contrasts. Winifred Strange, Linda Polka, and Manuela C. Aguilar (Speech Perception Laboratory, Department of Communication Sciences and Disorders, University of South Florida, Tampa, FL 33620-8100)

Previous research has shown that the Hindi retroflex-dental contrast among stop consonants is not easily differentiated by English. speakers even after some training. In the present study, subjects were given 4 days of training ( 768 trials) in a categorial AX discrimination task on full (unmodified) or truncated tokens of naturally produced breathy-voiced $\left[\mathrm{d}^{\mathrm{h}} \mathrm{a}\right] \mathrm{vs}\left[\mathrm{d}^{\mathrm{h}} \mathrm{a}\right.$ ]. Interstimulus intervals (ISI) were either short $(550 \mathrm{~ms})$ or long $(1500 \mathrm{~ms})$. Conditions favoring auditory-processing strategies (short ISI and truncated syllables) yielded the most improvement during training. However, pre-test to post-test improvement in "phonetic-level" discrimination of the full syllables was not significantly different across the four training conditions, and there was no transfer to discrimination of the contrast in voiceless aspirated $\left[\mathrm{t}^{\mathrm{h}} \mathrm{a}\right]-\left[\mathrm{t}^{\mathrm{h}} \mathrm{a}\right]$ or prevoiced [da $]-[\mathrm{da}]$ stops. Large individual differences found in each training condition suggest that subjects' strategies may be more important than stimulus and task variables in predicting success in perceptual differentiation of nonnative contrasts. [Supported by NINCDS.] 
NN12. Training methods for the facilitation of Japanese students' perception of American English $/ r /$ and $\Lambda /$. Salvatore Miranda (Department of Linguistics, University of Connecticut, U-145, Storrs, CT 06269-1145), [AV: Melva Underbakke, Winifred Strange, and Theodore Micceri (Speech Perception Laboratory, Department of Communication Sciences and Disorders, BEH 255, University of South Florida, Tampa, FL 33620-8100)

This study investigated the effects of two types of discrimination training on the perception and production of $/ r /$ and $/ /$ by Japanese students of English. Training stimuli consisted of synthetic syllables drawn from three different ten-step $/ r /-/ /$ series: rock-lock, rake-lake, and rooklook. The prototype group received discrimination training using only the endpoint stimuli from each series (the clearest instances of $/ r /$ and $/ /$ ). The gradient group received discrimination training that began with the endpoints and proceeded gradually to stimuli near the (English) category boundaries. Control subjects received discrimination training on naturally produced tokens of $/ \mathrm{b} / \mathrm{vs} / \mathrm{v} /$ (another contrast that Japanese find difficult). Comparisons of pre-test and post-test scores showed improvement for both prototype and gradient groups in identification of synthetic speech series and in production, but not in perception of naturally produced minimal pairs. Improvement for the control group was restricted to the perception of the synthetic $/ r /-\Lambda /$ series. No differences were found in the overall effectiveness of prototype versus gradient training. [Supported by NINCDS.]

\section{3:39}

NN13. Perceptual characteristics of English syllable-initial $/ r, l /$ for Japanese listeners. Reiko Yamada, Yoh'ichi Tohkura, and Noriko Kobayashi (ATR Auditory and Visual Perception Research Laboratories, Inuidani, Seika-cho, Kyoto 619-02, Japan)

This study investigates the perceptual characteristics of American English $/ r, l /$ for Japanese listeners using synthesized stimuli. Five major findings are obtained. (1) The Japanese listeners identify the stimuli using a variety of acoustic cues, and their response patterns are strongly influenced by acoustic features of the stimuli. In contrast, the American listeners can identify $/ r /$ and $/ /$ as long as a primary cue remains, even under the condition where some of the acoustic cues are missing. (2) As the Japanese listeners tend to perceive some stimuli as / $w /$ more than American listeners do, perception experiments with $/ w /$ as well as $/ r /$ and $\Omega /$ for a choice of identification better clarify the perception mode of the Japanese listeners. (3) A positive relationship between the identification ability of the natural $/ r, l, w /$ spoken by native Americans and that of the synthesized $/ r, l, w /$ is found for the Japanese listeners. (4) Contextual effects in words are very strong for the Japanese listeners when trying to identify $/ r /$ and $/ /$. (5) The Japanese listeners who have lived in Englishspeaking countries before a certain age are able to identify $/ r /$ and $/ /$ as well as native Americans.

\section{$3: 51$}

NN14. Perceptual categorization of synthesized $/ \mathbf{R}-\mathbf{W} /$ continua in normal preschool children. Laurie Fitzgerald and Elzbieta B. Slawinski (Department of Psychology, The University of Calgary, Calgary, Alberta T3A 1N4, Canada)

The purpose of the present study was to examine the development of categorical perception of phoneme boundaries. A seven-step adult /R-W/ continua was synthesized via the Klatt cascade/parallel software program. The acoustic tokens varied according to the second $(F 2)$ - and third $(F 3)$-formant onset frequencies and second $(F 2)$ - and third $(F 3)$ formant transition rates. Percentage of correct responses on an identification task was computed to yield a measure of phonemic boundary location for adults and children of 3,4 , and 5 years of age. The phonemic boundaries fell between stimuli 3 and 4, at stimulus 4 and 5 for 3-, 4-, and 5-yearold children and adults, respectively. The finding that there was a shifting and increasing steepness in the phonemic boundaries as a function of age is supportive of previous research and moreover, of the theory that a child's phonological system is not inherently different than that of an adult but rather is just a simpler or less precise version of the mature system.
NN15. Spectral slope as a cue for the perception of breathy and nonbreathy stops in Shanghainese. Nianqi Ren and Ignatius G. Mattingly (Haskins Laboratories, New Haven, CT 06511 and Department of Linguistics, University of Connecticut, Storrs, CT 06269-1154)

In Shanghainese, breathy excitation following release distinguishes category 2 (so-called "voiced unaspirated") stops from category 1 ("voiceless unaspirated") stops. The most obvious acoustic correlate of breathiness is relative spectral slope: During the first $50-100 \mathrm{~ms}$ after release, $\mathrm{H} 1-\mathrm{H} 2$ is greater for category 2 than for category 1 in both wordinitial and word-medial (morpheme-initial) stops (Ren, unpublished). To investigate the perception of breathiness, a series of synthetic disyllables was prepared in which the spectral slope during the $100 \mathrm{~ms}$ following the release of the word-medial stop was increased step by step increasing the value of the "open quotient" in the computation of the glottal waveform-a technique previously used for the synthesis of breathy vowels [C. Bickley, Work. Papers MIT Speech Commun. Lab. 1, 71-81 (1982)]. Other potential cues (closure duration, pulsing during closure, tone contour) were neutralized. Ten Shanghainese speakers labeled the stimuli with greater spectral slope as category 2 and those with smaller spectral slope as category 1. [Work supported by NICHD Grant 01994.]

\section{4:15}

NN16. The separation of two voicelike signals. P. G. Vaidya (Department of Mechanical and Materials Engineering, Washington State University, Pullman, WA 99164)

If two persons are talking or singing at the same time, human beings can selectively listen to only one of them, even if they are producing notes that are in unison. This paper presents an attempt to simulate this task by a computer. An elementary model of the voices of the two speakers has been used. Using this model, two voicelike signals are generated and mixed. The resulting signal is analyzed for the coherence of the transphase. Trans-phase is the phase difference between the components at two different frequencies when one of them has been sent through a nonlinear filter. If the trans-phase shows coherence, the signals are said to be transspectrally coherent. A justification for the assumption of such coherence in voicelike signals has been presented. The data for the transspectral coherence have been sent through a processor that yields an estimate of the individual sound separately.

\section{$4: 27$}

NN17. The role of auditory object formation in sentence perception. Thomas D. Carrell (Communication Sciences and Disorders, Northwestern University, Evanston, IL 60208-3570)

Previous work has demonstrated that amplitude-modulated timevarying sinusoidal (AM-TVS) replicas of natural sentences were more intelligible than simple time-varying sinusoidal (TVS) sentences [T. D. Carrell, J. Acoust. Soc. Am. Suppl. 184, S158 (1988)]. The goal of the experiments reported here was to determine the cause of this increased intelligibility. One potential explanation was based on the fact that AMTVS sentences were rated more natural (i.e., more humanlike) than TVS sentences. This increased naturalness might have increased the probability that speech-specific analysis was brought to bear in decoding the incoming message. The second potential explanation was based on the idea that the comodulation of the three component tones of a TVS sentence might cause them to be grouped together as an auditory object for further processing. It has been suggested that "....amplitude modulation helps form auditory objects from complex sound fields" [W. A. Yost and S. Sheft, J. Acoust. Soc. Am. 85, 848-857 (1989) ]. Evidence will be presented in favor of the second explanation. Specifically, the increase in intelligibility will be shown to be related to the comodulation of the three component tones and possibly to the mechanism underlying comodulation masking release (CMR). These findings suggest that the comodulation of the three tones comprising a TVS sentence create an auditory object and that auditory object formation is important in fluent speech understanding. 


\title{
Meeting of Accredited Standards Committee S1 on Acoustics
}

to be held jointly with

\section{Technical Advisory Group Meeting for ISO/TC 43 Acoustics, and IEC/TC 29 Electroacoustics}

\author{
D. L. Johnson, Chairman S1 \\ Larson-Davis Laboratories, 1681 West 820 North, Provo, Utah 84061
}

\begin{abstract}
Standards Committee S1 on Acoustics. Working group chairs will report on their progress in the preparation of standards on methods of measurement and testing, and terminology, in physical acoustics, electroacoustics, sonics, ultrasonics, and underwater sound. Work in progress includes measurement of noise sources, noise dosimeters, integrating sound-level meters, and revision and extension of sound level meter specifications. Open discussion of committee reports is encouraged.

The international activities in ISO/TC 43 Acoustics, and IEC/TC 29 Electroacoustics, will also be discussed. The Chairs of the respective U.S. Technical Advisory Groups for ISO/TC 43 (H. E. von Gierke), and IEC/TC 29 (V. Nedzelnitsky), will report on current activities of these Technical Committees. ISO/TC 43 and IEC/ TC 29 will next meet in Copenhagen during April 1990.
\end{abstract}

\section{Session OO. Underwater Acoustics VI: Arctic Ocean Acoustics}

Michael J. Buckingham, Chairman

Mission Management Department, Royal Aerospace Establishment, Farnborough, Hampshire GU14 6TD, England

Chairman's Introduction-2:00

Invited Papers

2:05

O01. Physical bases for Arctic ambient noise. Ira Dyer (Department of Ocean Engineering, MIT, 5-212, Cambridge, MA 02139)

The fundamentals of noise generation by ice fracture processes such as occur in the Arctic Ocean are reviewed. Noise events, presumably associated with individual ice fractures, are measured and studied in terms of their source strengths, characteristic frequencies, event signatures, event densities, and ice morphologies. A usefully narrow set of possible mechanisms seems to be emerging as a plausible explanation for a wide variety of cases. [Work supported by ONR Arctic Acoustics Program.]

\section{2:25}

O02. Experimental measurements of normal incidence reflection/scattering from undeformed natural sea ice. R. Francois, K. Williams, T. Wen, and P. Mourad (Applied Physics Laboratory, College of Ocean and Fishery Sciences, University of Washington, Seattle, WA 98195)

Using narrow-band pulses, experimental measurements of normal incidence reflection/scattering from the ice/water interface of naturally growing first year sea ice have been made. The results cover the frequency range from $20-300 \mathrm{kHz}$. A sufficient number of realizations have been obtained, using different experimental 
techniques, that generalization of the results can be made. The techniques involve monostatic experiments, ensonifying several different diameters of cylindrical ice surfaces, and undeformed areas of ice, using transducers with a variety of directivities including omnidirectional. The growing, or skeletal, layer of ice results in a graded porous, rough surface that can be treated using Biot theory to obtain acoustic velocities, a continuous velocity profile for reflection, and Kirchhoff theory for scattering. The results indicate that the mean amplitude reflection coefficient follows the approximate equation, $R_{A}=0.40-0.157 \log (f)(f$ in $\mathrm{kHz}$ ), in the transition region from about $2-200 \mathrm{kHz}$. At lower frequencies, the reflection coefficient is determined by the bulk velocity of the ice (the velocity gradient in the skeletal layer being unimportant); at high frequencies, the surface roughness and ice/water surface impedance mismatch may both contribute to the mean reflection coefficient observed. [Work supported by Office of Navy Technology with technical management by NORDA.]

OO3. Acoustic scattering by laboratory scale modeled sea ice. Orest I. Diachok (Naval Research Laboratory, Washington, DC 20375) and Robert M. Kille (American University, Washington, DC 20016)

Laboratory scale-modeled experiments were designed to provide highly controlled data for evaluation of existing and guidance for new theories of acoustic scattering from Arctic sea ice. Results from previously reported measurements of scattering loss from parallel and randomly oriented sea ice ridges situated on a plate between air and water (McClanahan and Diachok) will be reviewed and new results of scattering (particularly backscattering) versus angle at both high and low frequencies will be presented. Implications for acoustic remote sensing of geometrical properties of sea ice at great ranges in the Arctic will be discussed.

3:05

O04. Low-frequency scatter from a thin rough elastic plate: Theory and experiment. Ivan Tolstoy (Knockvennie, Castle Douglas DG7 3PA, SW Scotland)

The generalized smoothed boundary condition theory, based upon an original idea of Biot's [M. A. Biot, J. Acoust. Soc. Am. 44, 1616-1622 (1968) ], and developed after 1979 by Tolstoy, offers a flexible and straightforward technique for obtaining simple, formal solutions for low-frequency sound scatter from distributions of scatterers of arbitrary shapes and properties of any type at liquid/liquid or liquid/solid interface. In special cases such as a perfectly hard wall with perfectly hard bosses or cylindrical corrugations, the boundary condition enables one to recover well-known solutions. After a brief review of prior successful applications to the interpretation of rough boundary model experiments, it is shown how the method can be applied to the reflectivity and phase problems for a plane wave incident upon a thin rough plate satisfying the Germain equation on the surface of a liquid. The predictions of the theory are found to agree well with recently reported model work [T. P. McClanahan and O. I. Diachok, J. Acoust. Soc. Am. Suppl. 1 84, S218 (1988)]. [Work supported by NRL and ONR.]

\section{Contributed Papers}

OO5. Measurements of under ice broadband coherence. Roger W. Meredith (Naval Ocean Research and Development Activity, Arctic Acoustics Branch, Stennis Space Center, MS 39529)

The Arctic is a unique region acoustically, dominated by the under ice canopy. The ice varies seasonally in coverage, thickness, and roughness causing varying degrees of acoustic spatial and temporal variability. Horizontal coherence from explosive acoustic sources at a depth of $300 \mathrm{~m}$ under a relatively smooth Arctic ice pack is examined in the frequency range $100-1500 \mathrm{~Hz}$ over source-receiver ranges from $3-50 \mathrm{~km}$. Coherence estimation is a difficult problem and is not defined theoretically for transient broadband signals. Mean-square-coherence is computed using frequency band averaging and is computed separately for the direct and ice-reflected propagation paths. Coherence is found to fluctuate significantly with source-receiver range, and acoustic frequency for both acoustic propagation paths. The highest values of coherence do not always occur at the lowest frequency bands. Inside the acoustic half-channel, above $300 \mathrm{~m}$, coherence is less for the ice-reflected arrival. Changes in coherence due to under ice reflection are smallest for the $100-$ to $200-\mathrm{Hz}$ octave band, indicating less coherence loss due to under ice scattering, but the change is sometimes as high as $50 \%$. [Work supported by the Office of Naval Technology. ]

\section{$3: 40$}

006. Measurements of low-frequency ambient noise in the Greenland Sea. Michael J. Buckingham (Mission Management Department, Royal Aerospace Establishment, Farnborough, Hampshire GU14 6TD, England)

Sonobuoy measurements of omnidirectional ambient noise spectra have been made at several positions between the Denmark Strait and the Fram Strait off the east coast of Greenland. Results from lines of buoys placed normal to the ice edge indicate that, below $200 \mathrm{~Hz}$, the noise level is essentially uniform across the edge, with no peak observed in the level at the interface between the ice cover and the open water. Noise levels tend to be insensitive to wind speed/sea state, showing a typical level of $83 \mathrm{~dB}$ re: 1 $\mu \mathrm{Pa}^{2} / \mathrm{Hz}$ at $100 \mathrm{~Hz}$. Usually, the buoy nearest the coast shows the highest spectral level, suggesting that coastal processes are perhaps the principal source of low-frequency ambient noise in the Nordic Seas. Several mechanisms could be responsible for the noise, one hypothesis being the movement of glaciers from the Greenland ice cap, at an elevation of $\approx 2000 \mathrm{~m}$, down towards the sea. Each glacier contains on the order of a billion tonnes of ice or more which grinds its way over rough, rocky terrain. This movement generates seismic waves in the ground which could couple into the ocean through the seabed: An interface (Scholte) wave may propagate along the sea bottom and radiate acoustic energy into the water column. 
a 1-km-long vertical array. Range and depth errors were less than $1 \%$ and 1 wavelength, respectively [E. Livingston and $O$. Diachok, J. Acoust. Soc.

007. Results of matched-field processing of low-frequency Arctic underice data. E. Livingston and $O$. Diachok (Naval Research Laboratory, Washington, DC 20375-5000)

Matched-field processing of low-frequency ( 11 to $23 \mathrm{~Hz}$ ) high signalto-noise ratio $\mathrm{cw}$ signals in an Arctic (under-ice) environment has produced high signal gain (within $\mathrm{l} \mathrm{dB}$ of theoretical) with good range and depth localization for frequencies of 17 and $23 \mathrm{~Hz}$, at a range of $260 \mathrm{~km}$ on
Am. 86 (1989) ]. At the lowest frquency, $11 \mathrm{~Hz}$, the range estimates and gain were within $1 \%$ and $1 \mathrm{~dB}$, respectively, but the error in the depth estimate was large, greater than three wavelengths. Replica fields were computed based on a measured but range-independent sound-speed profile and a constant bottom depth, whereas there was a major bathymetric feature along the propagation path. Possible causes for error in depth localization due to environmental mismatch in the computed replica fields at $11 \mathrm{~Hz}$ will be discussed.

\title{
Meeting of Accredited Standards Committee S3 on Bioacoustics
}

to be held jointly with the

\section{Technical Advisory Group (TAG) Meeting for ISO/TC 43 Acoustics, IEC/TC 29 Electroacous- tics, and ISO/TC 108/SC4 Human Exposure to Mechanical Vibration and Shock}

\author{
L. A. Wilber, Chairman S3
}

422 Skokie Boulevard, Wilmette, Illinois 60091

\begin{abstract}
Standards Committee S3 on Bioacoustics. The current status of standards under preparation will be discussed. In addition to those topics of interest, including hearing conservation, noise, dosimeters, hearing aids, etc., consideration will be given to new standards that might be needed over the next few years. Open discussion of committee reports is encouraged.
\end{abstract}

The international activities in ISO/TC 43 Acoustics, IEC/TC 29 Electroacoustics, and ISO/TC 108/SC4 Human Exposure to Mechanical Vibration and Shock will be discussed. The Chairs of the TAGs for ISO/TC 43 (H. E. von Gierke) IEC/TC 29 (V. Nedzelnitsky), and ISO/TC 108/SC4 (H. E. von Gierke) will report on current activities of these Technical Committees and Subcommittees. ISO/TC 43 and IEC/TC29 will next meet in Copenhagen during April 1990. TC 108/SC4 will meet in Milan in March 1990. 


\title{
Session PP. Physical Acoustics VII: Nonlinear Acoustics
}

\author{
Wayne M. Wright, Chairman \\ Physics Department, Kalamazoo College, Kalamazoo, Michigan 49007
}

Contributed Papers

3:00

PP1. A continuous-wave reflection bridge ultrasonic spectrometer constructed from off-the-shelf components. Ronald $K$. Sundfors (Physics Department, Washington University, St. Louis, MO 63130)

A versatile continuous-wave reflection bridge ultrasonic spectrometer has been developed. This spectrometer features simple tuning of the bridge element (commercial hybrid junction) for frequencies up to 100 MHz, a wide variation of applied acoustic power, simple computer control to lock frequency to any point of the acoustic response, diode and phase coherent detection, sensitivity equivalent to the best nuclear acoustic resonance spectrometers, and construction from off-the-shelf components. Use of the spectrometer as a $\mathrm{cw}$ spectrometer, sampled-cw spectrometer, and NAR spectrometer will be discussed.

\section{$3: 15$}

PP2. Effects of boundary conditions on the propagation and interaction of finite amplitude sound beams. Jacqueline Naze Tjøtta (Applied Research Laboratories, The University of Texas at Austin, Austin, TX 78713-8029 and Department of Mathematics, The University of Bergen, 5007 Bergen, Norway), James A. TenCate (Applied Research Laboratories, The University of Texas at Austin, Austin, TX 787138029), and Sigve Tjøtta (Applied Research Laboratories, The University of Texas at Austin, Austin, TX 78713-8029 and Department of Mathematics, The University of Bergen, 5007 Bergen, Norway)

Weak nonlinearity in the propagation and interaction of real sound beams in a lossless fluid is considered. Special emphasis is given to the effects produced by various boundary conditions at the sound sources and other bounding surfaces. Asymptotic formulas and numerical results are presented for the second harmonic, and for the scattered sum and difference frequency sound generated by two harmonic beams that intersect at an arbitrary angle. The results are derived from a general theory presented earlier [see Naze Tjøtta and Tjøtta, J. Acoust. Soc. Am. 83, 487-495 (1988) ], which is valid for any source separation and amplitude distribution. In situations where the parabolic approximation is not legitimate ( large angles, broad beams), properly accounting for the boundary conditions may be crucial. [Work supported by the IR\&D program of ARL:UT, ONR, and VISTA/STATOIL, Norway. ]

\section{3:30}

PP3. Effects of focusing on the scattering of sound by sound. Corinne M. Darvennes, Mark F. Hamilton (Department of Mechanical Engineering, The University of Texas at Austin, Austin, TX 787121063), Jacqueline Naze Tjøtta, and Sigve Tjøtta (Applied Research Laboratories, The University of Texas at Austin, Austin, TX 78713-8029 and Department of Mathematics, The University of Bergen, 5007 Bergen, Norway)

The effects of absorption on the scattering of sound by sound were discussed by the authors at the previous meeting [J. Acoust. Soc. Am. Suppl. 185, S5 (1989) ]. In the present paper, the effects of focusing are considered. The Khokhlov-Zabolotskaya-Kuznetsov equation is used to derive asymptotic results for the sum and difference frequency sound that is generated by the noncollinear interaction of focused sound beams radiated from displaced Gaussian sources. As in the case of unfocused primary beams, there are two main contributions to the nonlinearly generated sound field: pumped sound and scattered sound. Attention is devoted to the effects of focusing on the relative and absolute levels of the pumped and scattered waves. Strong focusing causes the pumped and scattered sound fields to broaden and overlap, which increases the difficulty of identifying the scattered sound. Numerical results are presented. [Work supported by ONR (CMD and MFH), IR\&D Program of ARL:UT, and VISTA/STATOIL (JNT and ST). ]

PP4. Nonlinear elastic response of shocked fused silica. Y. M. Gupta and M. P. Conner (Department of Physics, Washington State University, Pullman, WA 99164-2814)

Large amplitude, combined compression and shear wave measurements have been obtained in fused silica (Dynasil 1000) shocked to a peak compressive stress of $6 \mathrm{GPa}$. The in-material, particle velocity profiles are analyzed to obtain the longitudinal stress-density relation for uniaxial strain and shear wave velocities in the shocked state. The shear wave velocity decreases with compression to a density compression of $7.6 \%$, and then increases with compression. Bulk modulus, Poisson's ratio, and mean stress in the shocked state were determined from the experimental data. Comparison of the shock and hydrostatic compression results show that the fused silica displays reversible, shear-enhanced compaction. Although two of the three third-order elastic constants $\left(C_{111}\right.$ and $\left.C_{166}\right)$ can be calculated from the present data, the difficulty in describing the fused silica response in terms of third-order constants is discussed. A microscopic model to explain shear enhanced compaction is suggested. [Work supported by AFOSR, Grant No. 87-0081 under the DOD/URI program.]

4:00

PP5. Nonlinear sound scattering of crossed focused beams in the presence of turbulence-Angular measurements. Murray S. Korman (Department of Physics, United States Naval Academy, Annapolis, MD 21402)

Experiments are performed involving the interaction of mutually perpendicular crossed, ultrasonic beams overlapping (at a common focal point) and interacting in the presence of turbulence generated by a $d=0.64-\mathrm{cm}$-diam submerged water jet $\left(\operatorname{Re}=8.5 \times 10^{5}\right)$. Measurements of the radiated sum-frequency intensity, Doppler shift, and-spectral broadening are recorded from the scattered spectra. In the absence of turbulence, no sum frequency can be detected. The two cw primary beams $f_{1}=1.9 \mathrm{MHz}, f_{2}=2.1 \mathrm{MHz}$ ) are generated by individual transducer units of $15.2-\mathrm{cm}$ focal length. The receiver (a circular plane array located outside the interaction region) is positioned with its axis perpendicular to the jet at a distance of $30 \mathrm{~d}$ from the nozzle (the interaction region). Pre- 
viously, very good theoretical predictions of mean flow and rms turbulent velocity were determined from the Doppler shift and spectral broadening data from a similar crossed-beam scattering experiment (M. S. Korman and R. T. Beyer, J. Acoust. Soc. Am. 84, 339-349 (1988); 85, 611-620 (1989)]. The primary beams were generated by circular plane array elements. Results suggested that the interaction involved an averaging across a large region of flow. The localized crossed-beam interaction region allows turbulent flow properties to be predicted with reasonable spatial resolution. [Work supported by the Naval Academy Research Council.]

\section{4:15}

PP6. Reflection of a plane finite-amplitude pulse from a hard surface: Amplification factor comparison between analytic and simulation. Victor W. Sparrow (Department of Electrical and Computer Engineering, University of Illinois, Urbana-Champaign, IL 61801 and USA-CERL, P.O. Box 4005, Champaign, IL 61824-4005) and Richard Raspet (Department of Physics and Astronomy, The University of Mississippi, University, MS 38677)

The amplification factor for a sound wave impinging on a hard wall is the ratio of the acoustic pressure near the wall to the acoustic pressure at the same position with the wall absent. Linear acoustics gives the familiar wall amplification factor of 2 , pressure doubling. However, the factor is greater than 2 for finite-amplitude waves. For regular reflection, as opposed to Mach reflection, the factor has been predicted in the ideal gas case analytically. In this presentation the wall amplification factor predicted by a nonlinear acoustics finite-difference simulation will be compared with the analytic result of Pfriem. A short videotape will demonstrate the simulation via animation. [Computations performed at the National Center for Supercomputing Applications, Champaign, IL.]

\section{4:30}

PP7. Force anisotropy in resonant acoustic levitators. M. Barmatz, G. Aveni, and Judith L. Robey (Jet Propulsion Laboratory, California Institute of Technology, Pasadena, CA 91109)

In general, the forces positioning a sample in an acoustic levitator depend on direction, which is to say they are anisotropic. The anisotropy of the acoustic force for several classes of levitating modes in rectangular and cylindrical resonators has been investigated. The degree of anisotropy depends on the chamber geometry, mode that is excited, and properties of the sample. The force anisotropy has been studied as a function of these variables for single mode levitators. The force field anisotropy is evaluated for small displacements from equilibrium. Also, the anisotropic properties for the maximum positioning forces in the resonator that maintain the sample within the acoustic potential well have been studied. Optimum chamber dimension ratios for maximizing the latter force fields will be presented. Experimental measurements verify the theoretical optimiz- ation predictions. Finally, it is shown that there is an unexpected isomorphism between the force fields in rectangular and cylindrical positioning modes.

PP8. On supercritical incidence of a finite-amplitude plane dilatational wave in an elastic solid. Kun-Tien Shu and Jerry H. Ginsberg (School of Mechanical Engineering, Georgia Institute of Technology, Atlanta, GA 30332)

An earlier analysis [ K. T. Shu and J. H. Ginsberg, J. Acoust. Soc. Am. Suppl. 185, S139 (1989)] described nonlinear reflection and refraction phenomena of a finite-amplitude dilatational wave at suberitical incidence on a plane interface between two bonded solids. The present work extends the earlier description to cases where the angle of incidence exceeds the critical value. The incoming wave is assumed to originate from the slower medium, so two critical angles exist, associated with evanescence of either the transmited dilatational or shear wave. The finite-amplitude version of Snell's law indicates that the dependence of the phase speed of the incident wave on its instantaneous amplitude induces, in the case of evanescent waves, fluctuations in the phase velocity parallel to the interface and in the decay rate normal to the boundary. This effect mirrors the fluctuations in the transmission and reflection angles of propagative waves. A numerical algorithm is developed to evaluate reflected or transmitted waveforms at a specified field point. In the special case of incidence close to a linear critical angle, the finite amplitude Snell's law indicates that the corresponding wave fluctuates between propagative and evanescent properties within a single period. [Work supported by NSF.]

\section{5:00}

PP9. The role of higher-order acoustic nonlinearities in NDE. Laszlo Adler, Peter B. Nagy, Paul McGowan, and David Rypien (Department of Welding Engineering. The Ohio State University, Columbus, $\mathrm{OH} 43210$ )

The acoustoelastic method is a well-established way to obtain thirdorder elastic constants in bulk materials. Modifications are needed to extend this technique to obtain higher orders, e.g., fourth-order elastic constants, or to increase accuracy to adopt the technique for very thin layers, e.g., adhesive bonds. These higher-order nonlinearities are separated from the stronger first-order nonlinearities (associated with the thirdorder elastic constants) by the appropriate choice of polarization and harmonic analysis of dynamic acoustoelastic results. It has been shown that inclusion of the fourth-order elastic constants will lead to strengthrelated mechanical properties of the adhesive bond. [This work was supported by the Center for NDE operated by the Ames Laboratory, USDOE for the Air Force Aeronautical Laboratories/Materials Laboratory under Contract No. SC-89-168 with Iowa State University.] 


\title{
Session QQ. Physiological Acoustics V and Psychological Acoustics IX: Central Institute for the Deaf, S. Richard Silverman Lecture in Hearing and Deafness
}

\author{
Richard G. Stoker, Chairman \\ Central Institute for the Deaf, 818 South Euclid Avenue, St. Louis, Missouri 63110
}

Chairman's Introduction-4:30

Invited Paper

4:35

QQ1. Acoustic injury of the inner ear: Morphological and physiological perspectives. M. Charles Liberman (Department of Otolaryngology, Harvard Medical School and Massachusetts Eye and Ear Infirmary, 243 Charles Street, Boston, MA 02114)

Of the many approaches to the study of acoustic injury, one of the most fruitful has been to view the functional changes in the response properties of single auditory-nerve fibers. Since each cochlear afferent contacts only one inner hair cell, single-fiber responses provide a functional window onto a restricted portion of the cochlear duct. Through the use of intracellular labeling, which allows the cochlear origin of selected afferents to be precisely specified, correlations between structural and functional changes have been made on the single-cell level. Correlations so obtained have revealed the functionally important structural changes underlying acoustic injury of the inner ear. Morphological data from both the light-and electron-microscopic levels suggest that most sound-induced permanent threshold shifts can be accounted for quantitatively based on the loss of sensory cells and/or the damage to their stereocilia. Of all the structures in the inner ear, the stereocilia, and especially the "rootlets" that anchor them to the tops of the hair cells, appear to be the most vulnerable to permanent damage from acoustic overstimulation. The structural changes underlying reversible threshold shifts are clearly different from those underlying permanent loss, but exactly which structural changes underly the temporary losses are much less clear. Structure-function correlations in damaged ears have also helped define the contributions of inner versus outer hair cells to the response properties of auditorynerve fibers. Damage to inner versus outer hair cells results in strikingly different abnormalities in neural tuning, spontaneous activity, and maximal discharge rates. The nature of these differences suggests that auditory perceptual abilities would be very different given threshold shifts of identical severity but differing balance of damage to the two classes of sensory cells. 


\title{
Session RR. Physical Acoustics VIII: Ultrasonics, Photoacoustics, Optoacoustics, and Thermoacoustics
}

\author{
Anthony A. Atchley, Chairman \\ Physics Department, Naval Postgraduate School, Monterey, California 93943
}

Contributed Papers

8:00

RR1. The use of reciprocity to calibrate a resonant photoacoustic measurement. J. D. Maynard and Chang Yu (The Pennsylvania State University, University Park, PA 16802)

While the photoacoustic effect provides a powerful means of measuring very low optical absorptions with pulse lasers, obtaining an accurate calibration has been a problem. However, in a resonant photoacoustic measurement, a reciprocity calibration (which does not require replacing transducers) is readily accomplished. All that is required is that a reversible transducer be present in addition to the photoacoustic (laser) excitation and the usual photoacoustic receiver. By making only electrical measurements using alternately the laser and the reversible transducer for excitation, the absolute acoustic levels in the sample may be determined from the transducer voltage. By also measuring the quality factor $(Q)$ of the resonance from the frequency response, the power deposited by the laser is easily determined. A separate measurement of the incident laser power yields the optical absorption coefficient. [Work supported by the Office of Naval Research.]

\section{8:15}

RR2. A new resonant photoacoustic technique for measuring very low optical absorption in glasses and crystals. Chang Yu and J. D. Maynard (The Pennsylvania State University, University Park, PA 16802)

One of the most sensitive methods for measuring low optical absorption in glasses involves the photoacoustic effect. Conventional methods use high-power pulse lasers and attached piezoelectric transducers to obtain detectable acoustic signals, but this method is limited by noise and scattered light at the transducer. A new technique has been developed using a cw laser, which, although it has less power, may be modulated at the acoustic resonant frequency of the sample and thus generates an acoustic signal amplified by the quality factor $(Q)$ of the resonance. Since $Q$ 's of $10^{5}$ to $10^{6}$ are readily obtained, a cw laser with only $0.01 \%$ of the energy of a pulse laser may be used to obtain several orders of magnitude improved sensitivity. To increase the $Q$ and thus increase the sensitivity, the measurements are made in vacuum, the sample supports (three saphire spheres) are located at nodal lines with computer-controlled positioners, and the transducers are noncontact interdigital capacitors that sense the motion of a nearby dielectric sample or the fringe field of a nearby piezoelectric sample. The noncontact transducers also eliminate the problem of scattered light. [Work supported by the Office of Naval Research.]

\section{8:30}

RR3. Critical behavior of the ultrasonic attenuation and velocity and shear viscosity for the binary mixture of nitrobenzene- $n$-hexane. I. R. Abdelraziq, S. S. Yun, and F. B. Stumpf (Department of Physics and Astronomy, Ohio University, Athens, OH 45701-2979)

Ultrasonic velocity and absorption and shear viscosity as a function of temperature and frequency are reported for the binary mixture nitrobenzene- $n$-hexane in the homogeneous phase above $T_{c}$. The observed absorp- tion $\alpha_{c} / f^{2}$ vs $f^{-1.16}$ yields a straight line as predicted by the dynamic scaling theory of Ferrell and Bhattacharjee. The critical amplitudes of the thermal expansion and specific heat have been calculated using the twoscale factor universality relation. The adiabatic coupling constant $g$ is calculated and compared to the experimental value. The experimental values of $\alpha / \alpha_{c}$ for nitrobenzene- $n$-hexane are compared to the scaling function $F\left(\omega^{*}\right)$ and show a good agreement with the theory. The velocity for the system at the critical concentration above the critical temperature appears to linearly decrease with increasing temperature.

\section{$8: 45$}

RR4. Observation of optoacoustic amplitude in $\mathrm{CS}_{2}$ at high input energies. Stanley A. Cheyne (School of Mechanical Engineering, Georgia Institute of Technology, Atlanta, GA 30332) and Henry E. Bass (Physical Acoustics Research Group, The University of Mississippi, University, MS 38677)

The optoacoustic amplitude as a function of laser pulse energy has been measured. A nitrogen laser emitting UV ( $337 \mathrm{~nm}$ ) pulses $800 \mathrm{ps}$ in duration was used to excite liquid carbon disulfide $\left(\mathrm{CS}_{2}\right)$, which strongly absorbs the UV radiation. Measurements indicate an optical absorption coefficient of $370 \mathrm{~cm}^{-1}$ implying an optical penetration depth of $27 \mu \mathrm{m}$. The spatial profile of the pulse was an ellipse at the focal point with dimensions of $330 \times 90 \mu \mathrm{m}$. The energy coupled with the short penetration depth resulted in a high energy density $\left(532 \mathrm{~J} / \mathrm{cm}^{3}\right)$. This was varied by placing glass microslides in the beampath. The optoacoustic amplitude as a function of energy exhibited changes in slope as the liquid in the focal volume underwent vaporization. The experimental observations were explained in terms of an energy-dependent coefficient of thermal expansion and optical absorption. [Work supported by the Office of Naval Research.]

\section{9:00}

RR5. The measurement of thermoacoustic phenomena using thermoacoustic couples. Anthony A. Atchley, Thomas J. Hofler, and Chia-ning Ao (Physics Department, Naval Postgraduate School, Monterey, CA 93943)

The results of measurements of thermoacoustically generated temperature gradients in thermoacoustic couples (TACs) subjected to acoustic standing waves are reported. The value of the temperature gradient is a function of the acoustic pressure amplitude, mean gas pressure, type of gas, TAC construction, and its position in the standing wave. Measurements were made with a computer-controlled apparatus for drive ratios (the ratio of acoustic pressure amplitude to mean gas pressure) from approximately $0.1 \%$ to $2.0 \%$, in argon and helium having mean pressures from approximately 0.1 to $0.3 \mathrm{MPa}$, for three different TACs as a function of their positions in the standing wave. The results are compared with predictions based on a theory by Wheatley et al. [J. Acoust. Soc. Am. 74, 153-170 (1983) ]. Three distinct regions of behavior are apparent over the 
range of drive ratios investigated. For drive ratios less than approximately $0.4 \%$, there is overall good agreement between theory and measurement. For drive ratios between approximately $0.4 \%$ and $1 \%$, the agreement diminishes almost linearly with increasing drive ratio. For drive ratios greater than approximately $1 \%$, irregularities appear in the temperature difference data series and the discrepancy between theory and measurement generally remains approximately constant, although some variations occur. [Work supported by ONR and the NPS Research Program.]

\section{9:15}

RR6. Laser ultrasonic measurements in highly porous materials: Sintering of ceramics. K. L. Telschow, J. B. Walter, and G. V. Garcia (Idaho National Engineering Laboratory, EG\&G Idaho, Inc., Idaho Falls, ID 83415-2209)

The use of lasers for both generation and detection of elastic waves in materials has opened the possibility of real-time monitoring of material elastic parameters during processing. At the INEL, a laser Doppler interferometer has been constructed that allows direct measurement of elastic waves from rough and/or optically diffuse surfaces. This apparatus, coupled with a pulsed laser for elastic wave generation, is currently being used for totally noncontacting, real-time, monitoring of the sintering of ceramics. Results will be presented on the densification of materials as a function of sintering time and temperature. Both the longitudinal and shear wave velocities in these materials have been found to vary approximately linearly with the densification up to nearly total theoretical density. Mechanisms of the generation and detection of these elastic waves, as well as propagation in porous materials, will be discussed. [This work was sup- ported by the Department of Interior's Bureau of Mines under Contract No. J0134035 through Department of Energy contract No. DE-ACO7. 76IDO1570.]

9:30

RR7. Ultrasonic behavior in an amorphous superionic conductor $(\mathbf{A g I})_{x}\left(\mathrm{AgPO}_{3}\right)_{1-x}$. Jiankai Hu, Senkui Zhang, and Qianlin Zhang (Department of Electronics, University of Science and Technology of China, Hefei, Anhui, People's Republic of China)

Amorphous superionic conductor $(\mathrm{AgI})_{x}\left(\mathrm{AgPO}_{3}\right)_{1-x}$ series samples have been obtained by means of the liquid-nitrogen rapid-quenching technique. In the series of glasses, for $\mathrm{AgI}$ with mole concentration of $x=0.45,0.5,0.55$, and 0.6 , the attenuation and velocity of longitudinal waves were measured by an ultrasonic pulse-echo method, in the temperature range $100-300 \mathrm{~K}$ at frequencies of 5 and $10.5 \mathrm{MHz}$. The temperature of the attenuation peak fell with increasing AgI content and decreasing frequency. In addition, the amplitudes of the attenuation peaks increased significantly with increasing AgI content, despite the fact that the total concentration of $\mathrm{Ag}^{+}$ions is about constant for this series of samples. The sound velocity of the sample $x=0.5$, at frequency $5 \mathrm{MHz}$, decreased monotonously with increasing temperature and had a small anomaly at $204 \mathrm{~K}$. The sound velocity of the glasses decreased slowly as $x$ was increasing at room temperature. The experimental results were fitted well to the unified theory of low-frequency fluctuation, dissipation, and relaxation process, i.e., the infrared divergence response theory proposed by Ngai [K. L. Ngai, Comments Solid Phys. 9, 127 (1979); also, Phys. Rev. B 20 2475 (1979)]. [Work supported by NSF of China.]

\title{
Session SS. Psychological Acoustics X: Central Processes in Auditory Detection, Discrimination, and Recognition
}

\author{
Blas Espinoza-Varas, Chairman \\ University of Oklahoma Health Sciences Center, Oklahoma City, Oklahoma 73190
}

Chairman's Introduction-8:30

Invited Papers

8:40

SS1. Basic sensitivity on vowel and consonant continua: A function of phoneme categories. Bert Schouten and Arjan van Hessen (Research Institute for Language and Speech, University of Utrecht, Trans 10, 3512 JK Utrecht, The Netherlands)

The relation between labeling and discrimination of stimuli drawn from a short-vowel and a plosiveconsonant continuum was investigated. Stimuli were generated by interpolation between the spectral envelopes (derived with cepstral deconvolution) of naturally spoken syllables. The tasks were phoneme labeling, fixed and roving same-different discrimination with or without feedback, and fixed and roving 2IFC discrimination with feedback. The results indicate that, with natural speech sounds, labeling precedes the discrimination response, even in fixed discrimination, which is supposed to measure basic sensitivity. Equivalence between labeling and discrimination was strongest with consonants, which agrees with the old categorical perception literature. Varying ISI had a small effect on fixed same-different discrimination; at $100 \mathrm{~ms}$, performance was relatively high, and significant category peaks had disappeared; this could suggest basic sensitivity, but labeling performance was even higher. The effect of ISI on 2IFC discrimination was the reverse: a long ISI of $2000 \mathrm{~ms}$ improved performance considerably, especially at the category peak. It seems, therefore, that with speech 2IFC is a labeling-oriented task: It improves with time, particularly in a fixed-discrimination paradigm. 
SS2. A performance-oriented approach to the hearing of complex sounds: Nonspeech and speech. Charles S. Watson (Department of Speech and Hearing Sciences, Indiana University, Bloomington, IN 47405)

Two general questions have been addressed with experiments using word-length tonal sequences, or "patterns": to what degree can the discriminability of complex auditory patterns be predicted from listeners' performance with simpler stimuli, especially single tones presented in isolation? and, (2) How do listeners' abilities to extract information from complex nonspeech sounds compare to their abilities to resolve the details of the waveforms of speech? The answers appear to be consistent with the results of comparable investigations conducted for the past half-century in the field of vision. Very little degradation in sensitivity or resolving power for components of complex sounds can be attributed to the complex stimulus context per se, aside, of course, from temporal masking. When patterns vary from trial to trial, selective attention and accurate discrimination performance can be achieved only when prior experience has made them so familiar that processing has become "automatic." Listeners' remarkable resolving power for speech sounds can readily be imitated by overtraining them on the discrimination of at least some nonspeech sounds.

SS3. Auditory profile analysis. David M. Green (Psychoacoustic Laboratory, Department of Psychology, University of Florida, Gainesville, FL 32611)

Data are reported on the ability of listeners to discriminate changes in the power spectra of complex acoustic signals. Since randomizing both the phase spectrum and the overall level of the signal on each presentation does not change detection performance, it is known that detection is not based on the signal's phase spectrum or the absolute level of energy at a particular frequency location. The discrimination must be based on a change in the shape of the power spectrum of the signal, what is called profile analysis. The most critical feature of profile analysis is that detection of a change at one spectral location depends on energy at very remote spectral locations. For example, the detection of an increment in the intensity of a component at $1000 \mathrm{~Hz}$ is influenced by the presence or absence of energy more than 2 oct away. Recent experiments show that these effects may depend on whether the standard power spectra are fixed or changing over successive trials, since spectra with randomly changing levels do not show such effects. Finally, some recent experiments will be discussed that provide estimates of the spectral weights employed by profile listeners, using a technique developed by Dr. Bruce Berg. [This research was supported by NIH and AFOSR.]

SS4. Relation between comodulation masking release and auditory grouping. Joseph W. Hall, III and John H. Grose (Department of Surgery, Division of Otolaryngology, University of North Carolina Medical School, Chapel Hill, NC 27599)

CMR was investigated in conditions where some flanking bands were comodulated with the on-signal band, but other flanking bands ("codeviant" bands) were comodulated between themselves, but not with the on-signal band. Past research has indicated that CMR is often substantially reduced when codeviant bands are present. It is possible that the disruptive effects of codeviant bands can be reduced by factors related to auditory grouping. In one condition, 20-Hz-wide comodulated bands were present at 400,600,800, 1000, 1200, 1400, and $1600 \mathrm{~Hz}$ ( $1000-\mathrm{Hz}$ signal frequency). When two codeviant bands were added at 900 and $1100 \mathrm{~Hz}, \mathrm{CMR}$ was reduced from 16 to approximately $4 \mathrm{~dB}$. Progressively more codeviant bands were then added, interspersed with the six comodulated bands. The rationale was that as more codeviant bands were added, they were more likely to be grouped separately from the on-signal band, and their disruptive effects would, therefore, be reduced. A second manipulation was to gate the codeviant bands differently from the comodulated bands: If the codeviant bands are given a unique gating pattern, they should be less likely to be grouped with the comodulated bands. Both of the above manipulations significantly reduced the disruptive effects of codeviant bands. The results suggest that one stage of CMR analysis apparently takes place subsequent to a stage of auditory grouping. [Work supported by the U.S. Air Force, AFOSR.] 
SS5. Factors in detection under uncertainty. Ervin R. Hafter and Robert S. Schlauch (Department of Psychology, University of California, Berkeley, CA 94720)

An especially effective tool for examining cognitive factors in hearing is the study of masking under conditions of uncertainty. Past studies of frequency uncertainty and its alleviation with cues leads to the proposal that the role of attention in detection is to govern the trade-off between the number of monitored bands and their effective bandwidths. In an extension of that work, attempts to measure the bandwidths directly and to understand the nature of cuing are made, whether it is a bottom-up process, as in the ringing of sensory filters, or top-down, as in the selection of the appropriate filter. Two distinct approaches are offered. In one approach, a modified "probe" technique presents a cue of either 1, 2, or 4 tones which includes, on most trials, the frequency of the signal; on probe trials, the signal is some fixed percentage (other than $100 \%$ ) of the frequency of one the cues. Performance based on data collapsed across equal percentages support the contention that changes occur in both effective number and bandwidth. Additional studies examine cues that require higher-order processing such as computation of musical interval or fundamental pitch. The effectiveness of these cues supports the notion of top-down processing. [Research supported by Grants from NINCDS.]

\section{1:00}

SS6. Central processes in the perception of pitch in simultaneous complex tones. John G. Beerends (P.T.T. Research, Neher Laboratories, P. O. Box 421, 2260 AK Leidschendam, The Netherlands)

Pitch identification of two simultaneous complex tones is more difficult than pitch identification of a single complex tone. In this talk, the more central processes that are involved in the perception of simultaneous complex tones will be discussed. Experiments are reported in which subjects had to identify two missing fundamental frequencies. A first set of experiments uses simultaneous two-tone complexes with different distributions of the four partial over the ears. It is shown that subjects can identify the two missing fundamental frequencies and that the identification results only weakly depend on the manner of distributing the four partials over the ears. For some subjects, the identification results showed that the more salient pitch can inhibit to some extent the identification of the less salient one. This "central interference" is most prominent when the partials of the two complex tones are widely separated. In a second set of experiments, the influence of signal duration on the virtual pitch of single and simultaneous complex tones is investigated. The results for single complex tones allow the formulation of a stochastic pitch model in which a central processor derives noisy subharmonics from noisy representation of the partials. For simultaneous complex tones, the behavior is more difficult to model because of the more central processes that are involved.

\section{$11: 25$}

SS7. Mechanisms of abstraction in auditory processing. Ernst Terhardt (Institute of Electroacoustics, Technical University, D-8000 Munich 2, Federal Republic of Germany)

The natural purpose of any sensory system is acquisition of information on external processes and objects, i.e., abstraction of the stimulus. Abstraction, in tum, is typically dependent on decisions that are based on internal knowledge. To get more insight into auditory information acquisition-including perception of speech and music-it is thus apparent that particular attention should be paid to decision processes on any level of the auditory system. Inspecting the auditory system from that point of view reveals that both its physical and neurophysiological design and its psychophysical behavior indeed exhibit many cues that are typical of abstraction mechanisms. For instance, extraction of individual "pure tones" from a continuous sound spectrum requires a decision mechanism; therefore, the formation of spectral pitches in the auditory periphery may be regarded as a first step of abstraction. On the basis of a number of experimental data and theoretical arguments, the following questions will be discussed: (1) How is the hierarchy of abstraction organized? (2) Where is the "sensory knowledge" stored? (3) What is the origin of that knowledge? (4) Which strategies of experimental research appear to be appropriate? 


\title{
Session TT. Speech Communication IX: Articulation: Measurements and Models
}

\author{
Ralph H. Ohde, Chairman \\ Vanderbilt University, School of Medicine, Division of Hearing and Speech Science, Box 562, Station 17, Nashville, \\ Tennessee 37232
}

Contributed Papers

\section{8:30}

TT1. Recovery of tongue function for speech and swallowing in partial glossectomy patients. Sandra L. Hamlet, Robin Patterson, and Susan Fleming (Department of Otolaryngology, Wayne State University, Detroit, MI 48201)

Most previous research on glossectomy speech has been cross sectional-patients were tested at only one point in time, usually several months or years post surgery. This study of oral cancer patients was designed to provide insight into the development of compensatory articulation and swallowing patterns in the early post surgery and subsequent radiation therapy period. Synchronized videofluoroscopic and acoustical recordings of speech and swallowing were made at specified times during and after cancer treatment. Tongue mobility relative to the jaw was greater post radiation treatment compared to post surgery. Expected co-articulatory variation in second formant frequency of vowels, as a function of consonant context, did not reappear until 6 months post treatment. Tongue activity for the oral phase of swallowing showed similar recovery of differential patterns related to bolus volume. Data on rule-governed subtleties of tongue activity related to details of the speech or swallowing context provide a sensitive measure of reestablishment of naturalness in tongue function.

\section{$8: 42$}

TT2. Cross-sectional tongue shapes and palatal contours during sibilant and lateral consonants. Maureen Stone (Department of Rehabilitation Medicine, Building 10, Room 6S235, N.I.H., Bethesda, MD 20892), Lawrence J. Raphael, and Alice Faber (Haskins Laboratories, 270 Crown Street, New Haven, CT 06511)

This presentation will examine the relationship between tongue shape and palatal contour during the production of $/ \mathrm{s} /, / \mathrm{S} /$, and $/ 1 /$. A combination of electropalatoraphy (EPG) and ultrasound (US) was used to compare tongue-palate contact patterns with their related cross-sectional tongue shapes. The phonemes $/ \mathrm{s} /, / \mathrm{s} /$, and $/ \mathrm{l} /$ are interesting to study because they are physiologically demanding. They necessitate distinct posterior tongue shapes to channel the air anteriorly (sibilants) or laterally $(/ /)$. In addition, these phonemes exhibit specific palatal contact patterns that result in their unique acoustic waveforms. Another effect of tongue-palate contact, which is usually overlooked, is the resultant bracing of the tongue. With the anterior tongue braced against the alveolar ridge, the posterior tongue is able to deform into a variety of cross-sectional shapes (concave for $/ \mathrm{s} /$, convex for $/ \mathrm{l} /$ ).

\section{8:54}

TT3. Construction of a three-dimensional tongue model. Reiner Wilhelms (Speech and Hearing Science Division, The Ohio State University, Columbus, $\mathrm{OH} 43210$ )

A three-dimensional model for the investigation of the dynamic behavior of the tongue is under study. Its purpose is to relate $\mathrm{x}$-ray microbeam-measured movements of pellets on the tongue surface to muscle activity in the tongue, and to generate area functions of the vocal tract in order to specify directly the mapping from muscle activity to acoustic output. As a first step in a finite element description, a reference model of the tongue is constructed that can be adjusted to different individual shapes and structures of the tongue muscles, based on anatomical studies. A schematic topology of the tongue is generated by separating a region of a Cartesian space into simple geometric shapes representing different tissue components. A mapping from this descriptive space to a curvilinear coordinate system using B-splines in three variables is specified to generate a realistic initial shape of the tongue. The orientation of muscle fibers can be determined by normalized gradient fields in the descriptive space.

\section{9:06}

TT4. Lip protrusion in sibilant production. Alice Faber (Haskins Laboratories, 270 Crown Street, New Haven, CT 06511)

While anecdotal reports of lip protrusion contributing to sibilant production in a variety of languages exist, little attention has been paid to quantifying its contribution. In the present study, lip position was monitored using a modified SELSPOT optoelectronic tracking system, and location of oral constriction was simultaneously monitored by means of a RION artificial palate. Subjects (speakers of Catalan, English, German, and Italian) produced 50 tokens each of $6(8)$ symmetrical VCV utterances, where $\dot{V}$ ranged over /iau(ü)/ and $\mathrm{C}$ over /šs/. As expected, /s/ has a more anterior constriction than $/ \check{\mathrm{s}} /$, with the constriction for /usu/ being the most anterior; and, there is more lip protrusion for $/ \check{s} /$ than for $/ s /$, except for in the $/ u_{-} u /$ context. Across utterance types, the combination of lip protrusion and constriction location is a better predictor of the frequency of a sibilant's centroid than is either parameter alone; within utterance types, lip protrusion and aperture are, in general, better predictors of centroid frequency than is constriction location. [Work supported by NIH Grant NS-07237.]

\section{9:18}

TT5. The front-cavity $/ F 2^{\prime}$ hypothesis tested by data on tongue movements. David J. Broad (1627 Bath Street, Apartment 3, Santa Barbara, CA 93101) and Hynek Hermansky (U.S. West Advanced Technologies, 6200 Quebec Street, Englewood, CO 80111)

The hypothesis that the second spectral peak $F 2^{\prime}$ resulting from perceptual linear prediction (PLP) corresponds to the resonance of the front cavity of the vocal tract is tested against data on tongue movements for non-low-back vowels. The front cavity is represented for this case as a uniform tube of length $L_{f}$. Scaling between movements of the tongue $\Delta x$ (range of a few centimeters) and of the vocal-tract constriction $\Delta L_{f}$ (range of about $10 \mathrm{~cm}$ ) is represented by an articulatory amplification factor $\alpha: \Delta L_{f}=\alpha \Delta x$. If $x$ is a tongue-surface coordinate parallel to the tract axis, then $1 / F 2^{\prime}=k+4 \alpha x / c$, where $k$ is a constant and $c$ is the speed of sound. When $x$ is the $x$ coordinate of a tongue-dorsum pellet tracked by $x$-ray microbeam, the correlations between $1 / F 2^{\prime}$ and $x$ are 0.74 and 0.75 for two speakers' triple repetitions of /wiw sju ji $/$. The respective estimates for $\alpha$ are 5.5 and 5.6. The correlations rise to 0.91 and 
0.92 if $x$ is a weighted sum of the $x$ and $y$ coordinates of three tongue pellets. Two predictions are thus confirmed: (1) a linear relation between $1 / F 2$ and tongue movements, and (2) reasonable values for $\alpha$. A more complex model of the front cavity may be needed to handle low back vowels. [The authors thank George Papçun of Los Alamos National Laboratory for access to his $\mathrm{x}$-ray data.]

\section{$9: 30$}

TT6. Interarticulatory relationships in vowel production. Mona Lindau and Peter Ladefoged (Department of Linguistics, UCLA, Los Angeles, CA 90024-1543)

In earlier studies it has been shown that, within a language (English), the jaw and the tongue interact in different ways for different speakers to produce differences between vowels. These studies were, however, limited to front vowels. Using the x-ray microbeam system, articulatory movements were recorded from pellets placed on the lips, mandible, and the tongue, in five speakers saying utterances illustrating all the English vowels. The results show some rather complex relationships. For example, for some speakers tongue height, as measured by the height of the tongue dorsum, does not always distinguish between the high front $/ \mathrm{i} /$ and mid front /e/ (e.g., in dee and day); but these vowels are distinguished by the height of the mandible. The height of the dorsum does separate the tense $\mathrm{h} / \mathrm{f}$ and the $\mathrm{lax} / \mathrm{h} /$ (e.g., in dee and $\mathrm{did}$ ) and the back $/ \mathrm{u} / \mathrm{and} / \mathrm{o} /($ e.g., in $d o$ and doe). The results also show the degree of consistency within individuals in favoring certain trading relationships between the lips, the mandible, and the tongue.

\section{9:42}

TT7. Articulatory and acoustic analysis of consonants differing in intelligibility. H. Timothy Bunnell (A. I. duPont Institute, 1600 Rockland Road, Wilmington, DE 19899)

Articulatory and acoustic recordings of a single talker producing multiple repetitions of nonsense utterances were made at the University of Wisconsin X-Ray Microbeam facility. The utterances consisted of the fixed frames "Say a Cawa" and "Say a waCa" with $C=\{/ \mathrm{b} /, / \mathrm{d} /, / \mathrm{g} /\}$. Each utterance was repeated three times in each of three speaking styles ranging from an exaggerated style intended to be "clear" (i.e., easier for the hard of hearing to understand) to a relaxed style intended to be "conversational." The audio recordings were presented to hearing impaired listeners in a three-alternative forced-choice stop consonant identification task to determine the relative intelligibility of the stop consonants. By hypothesis, the clearest consonants would be found in utterances that exhibited the greatest amounts of articulatory and acoustic change. However, this pattern was not found. For utterances with syllable-initial consonants (e.g., "Say a gawa"), actual intelligibility as estimated by percentage correct stop constant identification was inversely related to both the talkers intent and measures of the extent of articulator motion. Correlational analyses using both articulatory and acoustic measures to predict obtained intelligibility scores will be presented. [Work supported by NIH.]

\section{9:54}

TT8. Phase transitions in speech production and their perceptual consequences. Betty Tuller and J. A. Scott Kelso (Program in Complex Systems and Brain Sciences, Florida Atlantic University, Boca Raton, FL 33431)

Previous work shows that the relative phasing of articulatory events varies little across two levels of speaking rate and stress. But, one may ask, varies little with respect to what? A methodology is required that differentiates articulatory patterns as they evolve in time. With this aim, subjects were instructed to say /ip/ or /pi/ repetitively, at increasing speaking rates, while monitoring their glottal and lip movements. For /pi/, the observed interarticulatory phase relationships did not depend on speaking rate. For /ip/, relative phasing often changed markedly, suggesting that loss of stability underlies articulatory change (a phase transition). In two follow-up perceptual experiments, listeners appeared to judge the consonant's syllable affiliation on the basis of interarticulator relative phase, and did so in a "categorical" manner. These findings, interpreted in light of a theoretical model based on synergetics [Kelso et al., Physica Scripta 35, 79-87 (1987) ], may help resolve controversies concerning whether or not a particular variable is an articulatory "invariant." [ Work supported by NIH and NIMH.]

\section{0:21}

TT9. Speech movement coordination: Oral-laryngeal interactions. Vincent L. Gracco (Haskins Laboratories, 270 Crown Street, New Haven, CT 06511) and Anders Lofqvist (Department of Logopedics and Phoniatrics, University Hospital, S-221 85 Lund, Sweden, and Haskins Laboratories, 270 Crown Street, New Haven, CT 06511)

Kinematic investigations of speech motor actions have revealed elegant examples of coordinative motor control principles that reduce the degrees of movement freedom. For example, recent studies of upper lip, lower lip, and jaw movements for oral closure have demonstrated a temporal organization similar to rhythmic motor behavior such as locomotion. Consistent temporal coupling among multiple articulators is maintained and systematically scaled following unexpected mechanical perturbations of the lower lip or experimental manipulations of the phonetic environment. In order to generalize these apparent coordinative principles to include other phonetic environments and articulatory subsysterns, the present study investigated the adjustments underlying lip, jaw, and vocal and vocal fold movements associated with various voiceless sounds. Movements of the upper lip, lower lip, jaw, and vocal folds were recorded. Kinematic measures, including relative timing among jaw, upper/lower lip, and glottal opening/closing (devoicing) movements were obtained. Results demonstrate that coordinative timing relations among the multiple articulators are systematically scaled as a function of movement speed and phonetic context. Thus, consistent relative timing among functionally related articulators is a general property of speech movement coordination. It is hypothesized that such a constraint while simplifying the motor control process can provide sufficient kinematic variability while maintaining the underlying synergistic organization. [Work supported by NIH.]

$10: 33$

TT10. The kinematic of final lengthening in French. Janet Fletcher (Linguistics Department, 204 Cunz Hall, 1841 Millikin Road, Ohio State University, Columbus, OH 43210-1229) and Eric Vatikiotis-Bateson (Haskins Laboratories, 270 Crown Street, New Haven, CT 06511)

In a cross linguistic study of lip-jaw motion, Vatikiotis-Bateson (1988) showed that the kinematic patterns associated with the nonfinal accented-unaccented contrast in French are similar to those found for the stressed-unstressed contrast in English. Opening movements generally have longer durations, greater articulator amplitude, and higher peak 
velocity when accented than when unaccented. In the current study, the same French corpus is used to investigate final lengthening. Like English, French syllable duration is longer at the edge of intonational phrases than in preceding syllables. Yet, it is not clear what the articulatory correlates of this final lengthening are, nor to what extent they are language-specific. A recent study of final lengthening in English (Edwards, Beckman, and Fletcher, in press) showed that the extra lengthening of pitch accented syllables that are also phrase-final is accompanied by lower peak velocities in the latter part of the syllable with no change in articulator amplitude. However, initial results for French indicate that lengthening in phrasefinal syllables, which are always accented, is marked by higher articulator amplitude and peak velocity. Thus the kinematics of final lengthening is qualitatively similar to that of nonfinal accented syllables. The consequences for an articulatory and linguistic timing model of French will be discussed. [Work supported by Ohio State University, NSF, and NIH.]

10:45

TT11. Remote and local effects of stress within and among articulatory subsystems. Carol A. Fowler (Haskins Laboratories, 270 Crown Street, New Haven, CT 06511 and Dartmouth College, Hanover, NH 03755), Vincent Gracco, and Eric Vatikiotis-Bateson (Haskins Laboratories, 270 Crown Street, New Haven, CT 06511)

Previous research on stress has focused almost exclusively on articulatory correlates local to a single target syllable within a single articulatory subsystem (e.g., the lip-jaw or the respiratory system). However, the articulatory correlates of stress are not local in either sense. Although a few studies report remote effects of stress (effects not confined to the stressed segment) on the speech acoustics, articulatory studies have reported the effects of stress only for single subsystems (e.g., oral, laryngeal, and respiratory); there have been no systematic investigations of the extent to which stress effects are manifest throughout the speech production system. The present study investigates the effects of stress in the vocal tract and respiratory system. Lip and jaw and respiratory kinematics, laryngeal vibration, and intra oral air pressure were simultaneously recorded during recitation of sentences in which lexical, contrastive, or sentential (focus) stress were varied. Changes in the articulatory kinematics and in the relative timing of laryngeal, respiratory, and supralaryngeal activity are analyzed as a function of stress type and position in the utterance. Results suggest that the articulatory correlates of stress are distributed continuously within and across subsystems. [Work supported by NIH.]

\section{0:57}

TT12. Analysis of deaf speakers' breath stream dynamics during speech production. James J. Mahshie and Pradeep K. Yadav (Speech Communication Laboratory, Department of Audiology and SpeechLanguage Pathology, Gallaudet University, Washington, DC 20002)

Computer simulations have been used to characterize and better understand the articulatory behavior of normal [M. Rothenberg, Biblio. Phon. (1968); E. Muller and S. Brown, Speech Lang. (1980) ] and disordered [V. Gracco and E. Muller, ASHA 13 (1981)] speakers. In the present work, details of a computer simulation of breath stream dynamics of speech produced by deaf speakers will be presented. Oral airflow, oral air pressure, nasal airflow, electroglottograph, and acoustic data were acquired from both deaf and normal speakers during production of intervocalic bilabial plosives. Measures from these data were used to establish input parameters for simulation of both the deaf and normal-hearing speakers' utterances. Among the parameters manipulated in the model were: airflow resistance at the articulatory constriction, nasal tract resistance, vocal tract enlargernent, and glottal resistance. Pressure and flow outputs from both the simulations and speakers were compared. Advantages and limitations of the use of computer models to understand speech production processes of disordered speakers will be discussed. [Work supported by the Whitaker Foundation.]
TT13. In search of lingual stability. Eric Vatikiotis-Bateson (Haskins Laboratories, 270 Crown Street, New Haven, CT 06511) and Maureen Stone (Department of Rehabilitation Medicine, Room 6S235, Building 10, National Institutes of Health, Bethesda, MD 20892)

In this study, commensurate data were obtained for tongue motion (coronal and sagittal ultrasound scans), lingual-palatal contact patterns (dynamic palatography), and jaw motion (Selspot). Two subjects repeated / VCVCa/ combinations of $/ \mathrm{s}, \int, 1, \mathrm{i}, \mathrm{a}, \mathrm{o} /$. To date, the effects of consonant identity on jaw motion and palatal contact patterning have been examined, along with the anticipatory and carryover effects of the three vowels. Initial findings on both jaw height and palatal contact suggest that the tongue-jaw coupling observed for sibilant production was modulated systematically according to adjacent vowel identity. In contrast to $/ \mathrm{s} /$ and $/ \int /$, tongue-jaw coupling for $/ 1 /$ appears to be minimal as shown by the large token-to-token spatiotemporal variability of jaw motion. Instead, there is a very stable, yet highly asymmetrical pattern of lingual contact along the outer edge of the artificial palate. Analogous to the jaw's role in sibilant production, this may serve as a stabilizing brace for the tongue to achieve postures specific to $/ \mathrm{V} /$ production, which should be identifiable from analysis of the ultrasound data. These results are consistent with a task dynamic approach to interarticulator coordination [Saltzman (1986) ]. [Work supported by NIH.]

\section{$11: 21$}

TT14. Palatography: A comparison between custom-made and "flexible" artificial palates for speech production measures. Nancy $\mathrm{S}$. McGarr, Koichi Tsunoda (Haskins Laboratories, 270 Crown Street, New Haven, CT 06511 ), and Katherine S. Harris (Haskins Laboratories, 270 Crown Street, New Haven, CT 06511 and The Graduate School, City University of New York, New York, NY 10021)

While dynamic palatography, a method of measuring tongue-palate contact over time, has a long history, the method has seen limited use because of the need to custom-make a palate for each subject. Recently, the Rion Company has developed a "universal" semiflexible palate, with 64 contact points, in several sizes. This methodological study was designed to assess the comparability of data from flexible and custom-made palates, and the stability and reliability of flexible palate measures. Three speakers repeated multiple tokens of /isi/ and /asa/ with both palate types, and with flexible palates removed and replaced. While test-retest reliability was satisfactory for flexible palates, the custom-flexible comparisons revealed the need to develop measures of subject-to-subject differences in palate size and shape, if the technique is to develop its potential for large-scale studies of articulation in normal and clinical populations. Cornments will be made on analysis software, sampling rate, and contactpoint distribution. [Work supported by NIH Grant NS-13617.]

\section{1:33}

TT15. An evaluation of an alternating magnetic field device for monitoring articulator movements. Shuyong Shao, Betty Tuller, and J. A. Scott Kelso (Program in Complex Systems and Brain Sciences, Florida Atlantic University, Boca Raton, FL 33431)

The understanding of articulator coordination during speech has been limited by the type of data that can be safely gathered. Specifically, noninvasive methods of monitoring tongue motions are still not widely available. One promising method is an alternating magnetic field device to track movements of multiple articulators in the midsagittal plane. This report concerns one such commercially available device, the Articulograph AG-100. The principles of system design and operation have been reported previously by $\mathbf{P}$. Schonle and colleagues, in Germany, who developed the system's prototype. Here, an intensive evaluation of system 
accuracy, repeatability, and linearity, as well as measurements of tongue positioning during speech will be presented. These results indicate that with proper precautions in transducer calibration and placement, the Articulograph can be a useful tool in speech production research. [Work supported by NIH and NIMH.] and has to be trained to the articulatory data for the particular speaker. [Work supported by Ohio State University Speech and Hearing Department.]

\section{1:57}

TT17. Quantitative comparison of articulatory models. Michel T. T. Jackson (Division of Speech and Hearing Science, Ohio State University, Columbus, $\mathrm{OH} 43210$ )

\section{1:45}

TT16. Automatic alignment of phonetic events with $x$-ray microbeam articulatory data and the acoustic speech signal. J. H. Greenwald, A. K. Krishnamurthy, and O. Fujimura (Department of Electrical Engineering, The Ohio State University, 205 Dreese Labs., 2015 Neil Avenue, Columbus, OH 43210)

This paper describes an algorithm for the automatic alignment of phonetic events with $x$-ray microbeam articulation data and the corresponding acoustic signal. The algorithm uses a two-step procedure similar to that of Nelson [ Nelson et al., J. Acoust. Soc. Am. Suppl. 163, S32 (1978); Nelson, AT\&T Bell Laboratories Internal Rep. (1978)]. The first step locates the phrase boundary in continuous speech, and the second step matches the phonetic segments in each phrase. Articulatory and acoustic events are recognized in continuous speech, and matched to the predicted phonetic events using a dynamic programming technique. The place of articulation and voicing for certain phonemes are also matched with articulatory and acoustic events. The system is presently speaker dependent,
Phonological theories invoke a number of different articulatory parametrizations. Traditional phonetic theories and some phonological theories parametrize vowel articulation in terms of tongue height and backness. Particle phonology [S. A. Schane, Phonology Yearbook 1, 129-155 (1984) ] parametrizes vowel articulation as blends of particular (extreme) articulations $(i, u, a)$. Harshman, Ladefoged, and Goldstein [J. Acoust. Soc. Am. 62, 693-707 (1977) ] parametrize English vowels using front-raising and back-raising factors, each of which generates a family of tongue positions. A multispeaker, cross-linguistic database of $\mathbf{x}$-ray tracings [M.T.T. Jackson, UCLA Working Papers in Phonetics 71, 1-232 (1988) ] allows quantitative comparison of these proposals. Each proposal defines a vowel space: The coordinates of vowels in the vowel space can be fixed, and the articulatory correlates of the parameters using multivar iate regression can be estimated. Each model can, therefore, be evaluated by standard methods, e.g., $\chi^{2}$ tests. A number of such articulatory models have been investigated. The results suggest that front-raising and backraising factors generalize well across languages and fit articulatory data better than height/backness- or particle-based models.

\title{
Session UU. Underwater Acoustics VII: General Topies
}

\author{
Robert Hickling, Chairman \\ National Center for Physical Acoustics, University, Mississippi 38677
}

Chairman's Introduction-8:30

\section{Contributed Papers}

UU1. Abstract withdrawn.

UU2. Optimum bearing resolution for a moving towed array and extension of its physical aperture. Stergios Stergiopoulos (SACLANT Undersea Research Centre, Viale San Bartolomeo 400, 19026 La Spezia, Italy)

This paper examines the limits of the angular resolution capability of a moving towed array (MTA) by finding the Cramer $\sim$ Rao lower bounds (CRLB) and providing algorithms that extend the physical aperture of an MTA. The model that is considered for the CRLB estimates assumes that an $M$-hydrophone towed array is moving with known constant speed and that in the received signal unknowns are all the parameters for two 
sources. The estimated CRLBs for this model indicated that an $M$-hydrophone MTA provides very high angular resolution when the period $T$ of the received signal is very long. This ability of the moving array to resolve two closely spaced sources is related to the fact that the physical aperture has been extended by the distance traveled by the array during the $T$ seconds of the observation period. ( nputer simulation examples using the maximum likelihood estimator : d an extended towed array algorithm (ETAM) to find the bearing of the sources are presented. The results of these simulations agree with the CRLB if the signal-to-noise ratio (SNR) is higher than $0 \mathrm{~dB}$ at the hydrophone level of $-8 \mathrm{~dB}$ re: $1-\mathrm{Hz}$ band. Real data applications using the ETAM were successful, and the physical aperture of a 32-hydrophone MTA was extended to an equivalent of a 512-hydrophone fully populated array during $185 \mathrm{~s}$ of observation period. These results have also indicated that the performance of the above algorithm is very robust since it extends the physical aperture of an array by more than one order of magnitude for the case of a very low SNR broadband signal even though the source had 3.6-kn speed along its bearing.

\section{9:05}

UU3. A new method for passive synthetic aperture array processing. Edmund J. Sullivan (Naval Underwater Systems Center, Newport, RI 02841) and Stergios Stergiopoulos (SACLANT Undersea Research Center, APO New York, NY 09019)

A technique for extending the useful aperture of a towed array is presented. Its advantage over previous techniques is that it uses the existing aperture of the physical array to estimate the phase correction factor for the successive measurement. Thus the need for an accurate knowledge of the source frequency is avoided. By using the fact that, at any time, the array overlaps its position at a previous time, a maximum likelihood estimator can be constructed that computes the phase difference between the measurements taken at these two positions. This estimated phase difference is then used to correct the second measurement. An iterative procedure is then used to extend this technique over several measurements. This estimation procedure, along with estimating the kinematic phase correction factor $\omega \tau$, includes any other phase errors that may be incurred by such effects as array motion, propagation effects, or loss of temporal coherence. Results with both real and synthetic data are shown that demonstrate that the technique significantly outperforms previous techniques.

\section{9:20}

UU4. Inferring the shape of a towed array by processing the data from its acoustic sensors. Brian G. Ferguson (Maritime Systems Division, WSRL, DSTO, P. O. Box 706, Darlinghurst 2010, Australia)

When a horizontal linear array of underwater acoustic sensors is towed through the sea, motions within the water mass and variations in the tow vessel's heading can cause the shape of the array to deviate from a straight line. Distortion of the array's linear configuration can lead to degradation in the detection and localization performance of the array, unless the spatial processing of the acoustic data can compensate for the nonlinear shape of the array. One method for monitoring the shape of the array involves instrumenting the array with compasses and depth sensors. Another technique is presented here whereby the data from the acoustic sensors themselves are used to infer the spatial distribution of the sensors along the array.

\section{9:35}

UU5. Performance bounds for passive synthetic aperture sonar. Richard William Harvey (School of Information Systems, University of East Anglia, Norwich NR4 7TJ, United Kingdom)

The performance bounds for a towed line array are derived and compared with performance achievable using a static array. It is shown that the towed array can provide considerably better performance than the static array when the target is in the nearfield of the synthetic aperture.
The theoretical bounds have been validated by computer simulation and the simulation results for a least-squares estimator for various array/target geometries are presented. Methods by which a passive synthetic aperture might be implemented are discussed and the results of a least-squares estimator operating on $120 \mathrm{~s}$ of data recorded from a 10-element towed array are presented. [Work supported by SERC and Plessey Naval Systems.]

UU6. Acoustic navigation for a large aperture array. B. J. Sotirin ( $P-004$, Marine Physical Laboratory of the Scripps Institution of Oceanography, UCSD, La Jolla, CA 92093)

The acoustic navigation system for a large aperture $(900 \mathrm{~m})$ vertical array and the resulting data, describing the motion of the array and research platform FLIP during a 20-day deployment in the NE Pacific, are presented. Acoustic navigation data are acquired by detecting the highfrequency signals emitted by near bottom transponders. Recorded travel times are converted to $x y z$ positions by a nonlinear least-squares approach that adjusts the transponder, FLIP, and the array positions, minimizing the rms error. Simulation results highlight error sensitivities. The movement of FLIP, although constrained by a three-point moor, exhibits a wind-driven component and a clockwise semidiurnal tidal component with horizontal displacements in excess of $300 \mathrm{~m}$. Suspended vertically from FLIP under tension, the array has a damped response to tidal and internal wave forces. [Work supported by ONT.]

\section{0:05}

UU7. Time domain solutions for scattering from objects in a waveguide. Paul Ellis (School of Physics and Astronomy, University of Minnesota, Minneapolis, MN 55455) and M. F. Werby (School of Physics and Astronomy, University of Minnesota, Minneapolis, MN 55455 and NORDA, Numerical Modeling, Stennis Space Center, MS 39529)

Calculations that describe scattering from an object in a waveguide in the frequency domain are very time consuming. For the time domain it can be orders of magnitude more expensive both in time and storage than for the frequency domain, rendering such calculations impractical unless a supercomputer can be used. The solution of scattering from an object for a realistic target and waveguide using the Cray 2 computer (a 528Megaword, 4.1-ns machine) at the Minnesota Supercomputer Institute is discussed. The methods employed as well as results will be presented.

\section{0:20}

UU8. Scattering and transmission of sound power flow by solid elastic spheres in water. Robert Hickling, R. Kirk Burrows, James F. Ball, and Lou Ann Redmond (National Center for Physical Acoustics, University, MS 38677)

The scattering and transmission of sound power flow, or sound intensity, are calculated for plane waves incident on a solid elastic sphere in water. Since sound intensity is the same vector quantity in both the solid and the liquid media, the flow of sound power around and through the sphere can be treated as an entity. In addition to the flow of sound power, distributions of reactive intensity and potential and kinetic energy densities are computed. Extensive use was made of Mathematica in this work. [Work supported by the Office of Naval Research.]

\section{0:35}

UU9. Farfield projection from sparse nearfield measurement samples. Giorgio V. Borgiotti (Department of Electrical Engineering and Computer Science, George Washington University, Washington, DC 20052) and Eric Rosen (Naval Research Laboratory, Code 5130, Washington, DC 20375-5000)

The nearfield to farfield projection procedure here introduced has the following novel features. (1) The measured nearfield samples do not need 
to belong to a separable coordinate surface. (2) The number of samples can be as small as the theoretical number of degrees of freedom of the farfield, related to the dimensions of the source in wavelengths. (3) The samples do not need to belong to a regular grid. (4) If the projection is sought only within a limited angular sector, a further reduction of the number of measurement samples is possible. The technique is based on the identification from the measured data of an equivalent monopolar distribution of acoustic sources on a surface completely surrounding the radiator. The farfield is then straightforwardly computed. The identification is based on the pseudoinversion of the matrix representing the mapping of the equivalent sources into nearfield samples, which is obtained by resorting to the singular value decomposition of the matrix.

\section{0:50}

UU10. Impact of sample window choice and location on time delay estimation of transients. Allen E. Leybourne, III (University of Southem Mississippi, Box 5137, Hattiesburg, MS 39406) and Roger W. Meredith (Naval Ocean Research and Development Activity, Arctic Acoustics Branch, Stennis Space Center, MS 39529)

Estimation of time delays between coherent transient signals is biased by the choice and location of the sampling window. For transients that do not tend to zero within the sampling window (self-windowing), the uniform or boxcar window may not be the best choice due to spectral leakage. This is especially important when processing highly asymmetrical transient signals that are not self-windowing as in the case of acoustic ambient noise. When a sample window is used to suppress spectral leakage, a bias in time delay estimation results from a suboptimal window location. Even though the boxcar window produces the least bias in time delay when the transient is self-windowing, it is shown that sidelobe interference is signifcant for highly asymmetric signals. This work critically examines these effects and describes an iterative procedure to determine optimal location of the transient within any sample window. [Work supported by the Office of Naval Technology.]

\section{1:05}

UU11. Higher-order correlations and spectra and the detection of narrow-band deterministic transients. Lisa A. Pflug (NOARL, Stennis Space Center, MS 39529 and Department of Mathematics, University of New Orleans, New Orleans, LA 70148), Kenneth H. Barnes, George E. Ioup, Juliette W. Ioup (Department of Physics, University of New Orleans, New Orleans, LA 70148), Robert L. Field, James H. Leclere," and Grayson H. Rayborn (Department of Physics and Astronomy, University of Southern Mississippi, Hattiesburg, MS 39401)

Previous investigators have evaluated higher-order correlations and spectra for detection and time delay estimation of statistical signals. In this study detection of known and unknown deterministic transients, especially those which are narrow band, is considered. Initial results have been reported [G. E. Ioup, J. W. Ioup, K. H. Barnes, R. L. Field, J. H. Leclere, and G. H. Rayborn, IEEE Workshop on Higher-Order Spectral Analysis Proc., 46-55 (1989) ]. For narrow-band signals extending from frequency $f_{B}$ to $f_{T}$, the bispectrum is zero unless $2 f_{B} \leqslant f_{T}$ and the trispectrum has zero energy in the first and seventh octants unless $3 f_{B} \leqslant f_{T}$, although the remaining octants of the trispectrum are always nonzero. Conditions for adequate sampling for these spectra are presented. Moments and histogram analysis of the ordinate values of the transients are also used in the analysis of the detectors. Performance analysis using ROC curves shows that the simple bicorrelation detector does not do nearly as well as correlation or tricorrelation detectors for transients with $2 f_{B}>f_{T}$. Other performance analysis results are also discussed. [Work supported by NOARL and Navy/ASEE Summer Faculty Fellowship Program.] a) Also at NOARL.

\section{1:20}

UU12. Effect of pressure on sound absorption in seawater at $0{ }^{\circ} \mathrm{C}$. F. H. Fisher (University of California, San Diego, Marine Physical Laboratory, Scripps Institution of Oceanography, San Diego, CA 92152) and W. M. Wright (Department of Physics, Kalamazoo College, Kalamazoo, MI 49007)

Earlier measurements of the effect of pressure on sound absorption by magnesium sulfate in seawater by $\mathrm{Hsu}$ at $0^{\circ} \mathrm{C}$ in the laboratory were limited to pressures of just over 300 bars. The pressure was limited by the strength of the 100-liter titanium sphere, a spare ballast tank from the Woods Hole submarine A L VIN. To obtain data at higher pressures at $0^{\circ} \mathrm{C}$, measurements were recently made in $0.5 \mathrm{M}$ magnesium sulfate solutions in a cylindrical reesonator. Because the magnesium sulfate relaxation frequency is independent of pressure, measurements are necessary at only one frequency. This was done in the $100-\mathrm{kHz}$ region and a sodium sulfate solution was used to correct for wall losses in the cylinder. Mode interference problems as well as a shutdown in the building air circulation system, which gave rise to temperature control problems, degraded the data. Nevertheless, enough data were obtained at higher pressures (600 to 1300 bars) to be useful in assessing the effect of pressure on seawater sound absorption at $0^{\circ} \mathrm{C}$ as has been done with earlier work by Fisher at $25^{\circ} \mathrm{C}$ on 0.5 molar magnesium sulfate solutions. These results will be incorporated with those of $\mathrm{Hsu}$ at lower pressures into an equation for absorption in seawater at $0^{\circ} \mathrm{C}$ covering the whole pressure range. Predictions using this equation will be compared with those from the Garrlson-Francois and Fisher-Simmons equations for sound absorption in seawater. [This work was supported by Office of Naval Research.]

\section{1:35}

UU13. Geoacoustic models for bottom loss predictions. Charles W. Holland (Planning Systems, Inc., 7925 Westpark Drive, McLean, VA 22102)

A useful theoretical measure of acoustic interaction with the seafloor is the plane wave reflection coefficient $(R)$ which is commonly expressed as bottom loss (20 log $|R|$ ). Predictions modeling the seafloor as a sediment layer over a half-space indicate that bottom loss should be proportional to frequency. Bottom loss measurements $(50-1600 \mathrm{~Hz})$ in the deep ocean, however, frequently show a loss that is inversely proportional to frequency. The locations of these anomalous sites are remarkably consistent with the boundaries of the Abyssal Plains which are characterized by turbidite layering. Geoacoustic models including the turbidite layers were constructed based on core data and a ray-based acoustic model was employed to compare the model predictions with measured data in Abyssal Plain areas. The turbidite layering was modeled as a series of pelagic and terrigenous layers in a simple repeating sequence. Model predictions were in accordance with the measured data. 


\title{
Session VV. Physical Acoustics IX: Propagation and Porous Material
}

\author{
James M. Sabatier, Chairman \\ National Center for Physical Acoustics, P. O. Box 847, University, Mississippi 38677
}

Contributed Papers

\section{9:15}

VV1. Study of acoustic transmission loss in sediments at low frequencies using parametric acoustic arrays. V. N. Bindal, T. K. Saksena, S. K. Jain, Reeta Gupta, and D. N. Santoshi (Ultrasonic Section, National Physical Laboratory, New Delhi-12, India)

Investigations have been carried out on the acoustic transmission loss in clay, sand, and gravel in the frequency range $6-10 \mathrm{kHz}$. Parametric acoustic arrays have been used for this study and have been generated using amplitude modulation of a primary transducer of frequency about $300 \mathrm{kHz}$ in which the carrier was suppressed. Source level and beam width of the parametric signal have been $\sim 155 \mathrm{~dB} \mathrm{re:} 1 \mu \mathrm{Pa} / \mathrm{m}^{2}$ and $3.6^{\circ}$, respectively. Experiments have been conducted in the laboratory taking the sediment in a rectangular container of dimensions $0.7 \times 0.49 \times 0.6 \mathrm{~m}^{3}$. The acoustic transmission loss using parametric arrays has been compared with the measured loss with a nonparametric source. The results show a dependence of transmission loss on the particle size and are discussed in the light of previous studies.

\section{9:30}

VV2. Experimental and theoretical wave propagation in solid and hollow cylinders in fluid. Thomas J. Plona, Sergio Kostek, and ShuKong Chang (Schlumberger-Doll Research, Old Quarry Road, Ridgefield, CT 06877-4108)

A comparison between experiment and theory is made involving axisymmetric wave propagation along solid and hollow cylinders immersed in a fluid (i.e., water). The theoretical methods to generate waveforms include both integral representation and finite-difference modeling. The dispersive modal characteristics are calculated from the waveforms. The experiments are made on solid and hollow steel cylinders of $0.75 \mathrm{in}$. and $0.625 \mathrm{in}$. The source and receiver transducers are made from PZT rings 1.0 in. and 0.5 in. high which freely slide along the cylinders. Data are collected as a function of source-receiver spacing and over a frequency range of $40-240 \mathrm{kHz}$. A Prony's method is used to obtain the modal characteristics. Discussions will focus on the good agreement between theory and experiment, the differences between the mode structures in the two cases and the asymptotic behaviors at short and long wavelengths. Some interesting comparisons between monopole and dipole modes will also be made.

\section{9:45}

VV3. Transient response of a fluid-filled borehole due to the passage of transient elastic waves. C. Chang and S. K. Chang (Schlumberger-Doll Research, Old Quarry Road, Ridgefield, CT 06877-4108)

The interaction of transient elastic waves with a fluid-filled borehole is investigated both experimentally and theoretically. The elastic waves incident from various angles are partially scattered by the borehole, while an acoustic wave field is introduced in the borehole fluid. Experiments were conducted in a solid block with a circular borehole inside. The transient elastic waves were generated by using either a simulated acoustic emission source or an ultrasonic transducer. The acoustic pressure in the borehole was measured by a commercial PZT hydrophone and by a home-made PVDF hydrophone. The effect of the borehole on the elastic waves is identified by comparing the measured acoustic responses with the undistorted incident pulse, calculated by the generalized ray theory without the borehole. The theory of plane wave scattering by a borehole is used to compare with the experimental data. The transient results from both the experiments and the borehole theory agree with each other very well, and they both exhibit resonances due to the multiple reflections inside the borehole fluid.

\section{0;00}

VV4. Apparatus to determine the complex mass density of a viscous fluid contained in a rigid porous solid from acoustic pressure measurements. Robert A. Mirick,") Steven R. Baker, and Oscar B. Wilson (Department of Physics, Code 61Ba, Naval Postgraduate School, Monterey, CA 93943)

Two experimental techniques to determine the frequency-dependent complex mass density of a viscous fluid contained in a rigid porous solid are being investigated. The fluid-filled solid is contained within a small $(\ll \lambda)$ cylindrical cavity. In one technique the moving mass of the fluid is sensed by the effect it has on the measured input electrical impedance of a moving coil transducer. In the second technique the moving mass is extracted from the measured pressure required for the fluid to oscillate with a known amplitude through the porous solid. Preliminary results have been reported for the impedance method [Grant et al., J. Acoust. Soc. Am. Suppl. 184, S176 (1988) ]. Preliminary results for the pressure technique and a comparison of the two methods will be presented. [Work sponsored by NRL-USRD and the Naval Postgraduate School.] ${ }^{a)}$ LCDR, USN.

\section{0:15}

VV5. Measurement of acoustical and microstructural properties of real and model porous materials. Michael R. Stinson, Yvan Champoux, and Gilles A. Daigle (Division of Physics, National Research Council, Ottawa, Ontario K lA 0R6, Canada)

Detailed measurements of several parameters describing porous materials are currently being made. The acoustical behavior of the porous materials is determined using an impedance tube technique, modified to provide both characteristic impedance and propagation constant, as a function of frequency. To define the microstructural properties, flow resistivity and porosity are initially being measured. Several real materials are being considered, including samples of reticulated open-pore foam, felt materials, and natural soils. A series of model porous materials, with well-defined microstructure, is also being developed. The first in this series consists, simply, of circular pores of uniform cross section, formed in a rigid host material. The measurements will be interpreted in terms of existing rigid-frame models of the acoustical properties of porous materials. 
VV6. The slow compressional wave in a porous medium by the finite element method. Yu-chiung Teng (Aldridge Laboratory of Applied Geophysics, Columbia University, New York, NY 10027)

The finite element results for a fluid/porous-solid/fluid system are presented by using the double-nodel-layer technique. The pressure-type fluid elements are used to model the fluid fields: The porous solid is modeled by Biot's medium [J. Acoust. Soc. Am. 28, 168 (1956)]. The Biot's theory predicts that for propagation of acoustic waves in fluid-saturated elastic porous media, a second compressional wave exists. Geertsma and Smit [Geophysics 41, 169 (1961)], using Biot's theory, predicted the generation of this second type of compressional wave at an open interface between a liquid and a porous solid. Plona [Appl. Phys. Lett. 36, 259 (1980)] observed the second type of compressional wave in his liquid/ porous-solid/liquid experiment at ultrasonic frequencies. Given suitable physical parameters in the finite element calculations, excellent agreement with the Plona's measurement has been obtained. The finite element results for a sealed interface, showing a diminishing of the second type compressional wave, also agree with the experimental work performed by Rasolofosaon [Appl. Phys. Lett. 52, 780 (1988)].

\section{0:45}

VV7. Dependence of the acoustic to seismic coupling ratio on the angle of incidence and geophone depth. W. Pat Arnott, James M. Sabatier, and John O. Messer (National Center for Physical Acoustics, P. O. Box 847, University, MS 38677)

An atmospheric sound wave can couple with the poroelastic ground, resulting in ground (or seismic) motion. This is the phenomenon of acoustic to seismic coupling. Microphones are used to measure the sound pressure level at the surface and geophones are used to measure the resulting seismic motion at or below the surface. The seismic:acoustic transfer function (SATF) characterizes a particular site. SATF measurements are reported as a function of the angle of incidence of the sound wave for frequencies $15 \mathrm{~Hz}$ to $1 \mathrm{kHz}$. The angle of incidence varied from $50^{\circ}$ to $80^{\circ}$, and normal incidence. Surface vertical and radial-horizontal geophones were used. In addition six vertical geophones at depth intervals of $10 \mathrm{~cm}$, starting at $10 \mathrm{~cm}$, were used. A seismic $p$-wave survey indicated a first layer depth of $44 \mathrm{~cm}$ having a wave velocity of $159 \mathrm{~m} / \mathrm{s}$ overlying a layer of velocity $379 \mathrm{~m} / \mathrm{s}$. This gives a critical angle of incidence of $65^{\circ}$. It was anticipated and confirmed that the SATF would increase in magnitude for certain frequency bands as the angle of incidence approached and passed over the critical angle. Comparisons of experimental results and multilayered elastic and poroelastic media theory will be addressed as time permits. [Work supported by ONR.]

\section{1:00}

VV8. Experimental studies of low-frequency propagation over flat terrain. D. Keith Wilson and Dennis W. Thomson (Department of Meteorology, Pennsylvania State University, University Park, PA 16802)

Experimental studies of atmospheric acoustic propagation have included monitoring the level of a $27.7-\mathrm{Hz}$ source at a distance of $770 \mathrm{~m}$. Two of the extended duration experiments consisted of recording SPL at 1-min intervals for 75 h over dry ground (Oct. 1988), and for 144 h over frozen, snow-covered ground (March 1989). Comprehensive surface layer micrometeorological measurements, including monitoring the turbulent momentum, heat, and moisture fluxes adjacent to the path, as well as continuous Doppler sodar measurements of the boundary layer wind profiles, were made. These measurements are used to reconstruct the timedependent "along path" sound speed profiles. Interpretation of the acoustic data includes comparisons with theoretical predictions from a fast field program (FFP).
VV9. Low-frequency, long-range sound propagation modeling over a locally reacting boundary with the parabolic approximation. J. S. Robertson (Department of Mathernatics, U. S. M. A., West Point, NY 10996-1786), M. J. Jacobson, and W. L. Siegmann (Department of Mathematical Sciences, Rensselaer Polytechnic Institute, Troy, NY 12180-3590)

There is substantial interest in the analytical and numerical modeling of low-frequency, long-range atmospheric acoustic propagation. Raybased models, because of their frequency limitations, do not always give an adequate prediction of quantities such as sound pressure or intensity levels. However, the parabolic approximation method, widely used in ocean acoustics, and often more accurate than ray models for frequencies of interest, can be applied to acoustic propagation in the atmosphere. Modifications of an existing implicit finite-difference implementation for computing solutions to the parabolic approximation are discussed. A locally reacting boundary is used together with a one-parameter (the flow resistivity) ground impedance model. Intensity calculations are performed for a number of flow resistivity values in both quiescent and windy atmospheric sound channels. Variations in the value of this parameter are shown to have substantial effects on the spatial variation of the acoustic signal. [Work supported by NASA.]

\section{1:30}

VV10. An investigation of the relationship between upward refraction above a complex impedance plane and the spherical wave evaluation for a homogeneous atmosphere. Richard Raspet, Gordon E. Baird, and Wenliang Wu (Physical Acoustics Research Group, University of Mississippi, University, MS 38677)

A residue series solution based on Fock's work in electromagnetic propagation has been used by several investigators to examine sound propagation into a shadow zone. In this paper it is demonstrated that this solution merges smoothly into the Sommerfeld solution for sound propagation above a flat surface as the sound velocity gradient goes to zero. A principle thrust of this investigation is the behavior of the sound propagation above a complex impedance plane as the gradient becomes finite. Initial work indicates that the surface wave pole may contribute to the residue series solution under certain conditions.

\section{1:45}

VV11. Scattering of sound by atmospheric turbulence. Walton E. McBride, Henry E. Bass, Richard Raspet (Physical Acoustics Research Group, The University of Mississippi, University, MS 38677), and Kenneth E. Gilbert (National Center for Physical Acoustics, The University of Mississippi, University, MS 38677)

A computer simulation of the effect of small scale turbulence on atmospheric sound propagation over a complex impedance boundary is developed. The atmosphere is broken up into spherically symmetric eddies characterized by a Gaussian profile. Single scatter is assumed and a closed form of the first Born approximation for scattering is obtained, giving each eddie's contribution to the total fluctuation of the sound pressure at a receiver downrange. The numerical simulation was accomplished with the concept of a "realization," or snapshot of the turbulent medium. Each eddie's scatter contribution was added up for a particular configuration of eddies, giving that realization's total sound pressure fluctuation. The eddies were then given a random change in their coordinates. The total sound pressure was calculated for this realization, and the process repeated. A complex impedance boundary was added and the predictions of the standard deviations of the amplitude fluctuations, amplitude probability distributions, and structure functions were then tested against experimental data. Good agreement was found whenever the average intensity of the fluctuations was well above the background noise level. [Work supported by U. S. Army Construction Engineering Research Laboratory.] 


\title{
Session WW. Psychological Acoustics XI and Musical Acoustics IV: Perception of Complex Sound
}

\author{
Charles S. Watson, Chairman \\ Department of Speech and Hearing Science, Indiana University, Bloomington, Indiana 47405
}

Contributed Papers

1:00

WW1. Weighting functions in a multiple tone task. Bruce G. Berg (Psychology Department, University of Florida, Gainesville, FL 32611)

An analytical technique is used to assess the relative weight given to each observation in a multiple tone task. After listening to a sample of seven tones presented sequentially, observers try to discriminate if the tones were sampled from a "high" or "low" distribution. The frequency of each tone is determined by sampling from one of two Gaussian distributions with means of 1000 and $1100 \mathrm{~Hz}$. Reliability of each tone is manipulated by changing the standard deviation of the distributions as a function of temporal position. In experiment 1 , the three central tones have either equal, greater, or less reliability than the remaining tones. Observers adjust their weights appropriately. In experiment 2 , sequentially presented tones alternate between high and low reliability, and intensity cues their reliability. Observers adjust their weights appropriately when highly reliable tones have greater intensity, but not when highly reliable tones are cued by low intensities. Finally, in a two-tone experiment, only the first tone of fixed intensity is reliable. Unexpectedly, discrimination accuracy decreases as the intensity of the second, uninformative, tone is increased. [Work supported by AFOSR.]

1:15

WW2. Effect of multiple presentations on detection and recognition of pure tones and tonal patterns. Robert C. McKinley and Daniel L. Weber (Department of Psychology, Wright State University, Dayton, $\mathrm{OH}$ 45435)

This study examines the effect of multiple observations on detection performance, and on the ability to recognize one signal from a set of possible signals, for three different sets of four signals. One set is four pure tones (100-ms sinusoids, frequencies of $707,1000,1414$, and $2000 \mathrm{~Hz}$ ). Two sets contain tonal patterns each made of seven 100 -ms sinusoidal components. The first set of four patterns used different frequencies from different one-fourth octave bands (centered at 707, 1000, 1414, and 2000 $\mathrm{Hz}$ ) to produce a single pattern of relative frequencies in different frequency regions. The second set had different sequences of a single set of frequencies. All stimuli were adjusted to equal sensation level in the presence of a continuous white noise (spectrum level $20 \mathrm{~dB}$ SPL). Each trial had multiple presentations $(n=1,2,4,8$, or 16$)$ of a given signal plus noise (probability of a signal trial was 0.5 ) or noise alone. Detection performance for the pure tones and the first set of tonal patterns was well described by a square root of $n$ improvement; detection for the second set of patterns was not. Recognition performance was generally poorer than that predicted by the detection/recognition theorem [S. J. Starr, C. E. Metz, L. B. Lusted, and D. J. Goodenough, Radiology 116, 533-538 (1975)] and was not affected by multiple presentations other than as predicted by the increase in detection performance. [Research supported by AFOSR through AAMRL/BBA, Wright-Patterson AFB.]

\section{1:30}

WW3. Detection of relative-frequency changes in tonal sequences. Gary R. Kidd and Charles S. Watson (Department of Speech and Hearing Sciences, Indiana University, Bloomington, IN 47405)

It was previously reported that listeners' abilities to detect frequency changes in randomly generated sequences of tones were substantially worse when the changed sequence was transposed in frequency [G. R. Kidd and G. S. Watson, J. Acoust. Soc. Am. Suppl. 1 84, S14l (1988) ]. Listeners apparently rely on absolute frequency whenever they can and are relatively poor at using relative-frequency information even when they must do so to perform a discrimination task (i.e., when the changed pattern is transposed). The ability to detect changes in relative frequency was examined further in new experiments that included both high- and lowuncertainty conditions with five-tone and two-tone sequences. Under high-uncertainty conditions, performance with both five- and two-tone sequences was again relatively poor when the patterns were Iransposed, although somewhat better with the two-tone sequences. Under low-uncertainty conditions, performance with transposed patterns at both sequence lengths was often as good or better than with untransposed patterns, suggesting a strong role of pattern familiarity in the identification of patterns by the sequences of relative frequencies. (Work supported by AFOSR and NIH.]

\section{$1: 45$}

WW4. Fatigue effects of a pure tone and pure tones, I. M. Young and L. D. Lowry (Department of Otolaryngology, Jefferson Medical College of Thomas Jefferson University, Philadelphia, PA 19107)

Subjects with normal hearing were exposed to moderate intensity of a pure tone and pure tones. Exposure frequency consisted of: a single tone, $1500 \mathrm{~Hz}$; two tones, 1500 and $2000 \mathrm{~Hz}$; and three tones, 1500,2000 , and $3000 \mathrm{~Hz}$. Fatigue effect measurements were made by automatic audiometry immediately after exposure. The greatest fatigue at frequencies 1/2-1 oct above an exposure frequency of $1500 \mathrm{~Hz}$ was shown by a single tone, the least effect by three tones, and the intermediate effect by two tones. The fatigue effects at the remote high frequencies were in the reverse order. These findings may be explained on the basis of harmonics and combination tones arising in the ear on stimulation with tones.

\section{2:00}

WW5. Auditory-filter shapes at three test frequencies with a burst and a fringed notched-noise masker. Beverly A. Wright (Department of Psychology, University of Texas, Austin, TX 78712)

The shapes of the auditory filters centered at $0.5,1.0$, and $3.0 \mathrm{kHz}$ were determined using Patterson's notched-noise method, but with the onset of the tonal signal delayed either $1 \mathrm{~ms}$ (burst condition), or $350 \mathrm{~ms}$ (fringed condition) after the onset of the masking noise. The masking noise ranged from $0.1-10.0 \mathrm{kHz}$, and its spectrum level was $24 \mathrm{~dB}$. All waveforms were gated with a rise/fall time of $10 \mathrm{~ms}$, and the offset of the signal always preceded the offset of the noise by $2 \mathrm{~ms}$. Although there were individual differences across listeners, for all six of them, the filters at the three test frequencies were narrower in the fringed than in the burst condition. In general, the differences in the filter shapes in the two conditions increased as the signal frequency increased. In accord with unpublished work of $\mathbf{R}$. Carlyon, the narrowing process was complete with signal delays of 50 $100 \mathrm{~ms}$ and was observed for signal durations of 25 and $50 \mathrm{~ms}$, but not for a signal duration of $200 \mathrm{~ms}$. Possible underlying mechanisms will be discussed. [Work supported by NINCDS.] 
measures on threshold and signal level. Auditory-filter characteristics, critical ratios, psychophysical tuning curves, and masking patterns were

WW6. Frequency resolution for broadband-noise masked normal listeners. Judy R. Dubno and Amy B. Schaefer (Division of Head and Neck Surgery, 31-24 Rehabilitation Center, UCLA School of Medicine, Los Angeles, CA 90024-1794)

When evaluating frequency resolution of hearing-impaired listeners, it is often difficult to determine if changes observed in auditory analysis with increasing auditory threshold reflect abnormal cochlear function or represent normal auditory processing at high stimulus levels. This experiment was designed to investigate the dependence of frequency-resolution obtained from normal-hearing listeners in quiet and in the presence of broadband noise at five levels ( 30 to $70 \mathrm{~dB} S P L$ ). These findings were then compared to previously obtained frequency-resolution results for subjects with sensorineural hearing loss whose auditory thresholds in quiet were comparable to the normal-hearing listener's masked thresholds. These comparisons, while limited to individuals with moderate hearing loss and broadband-noise masked normal listeners, suggest that frequency resolution observed in hearing-impaired listeners is similar to that seen in the normal auditory system at high stimulus sound-pressure levels. [Work supported by NIH NS 18015.]

\section{$2: 45$}

WW7. Gap detection and discrimination in complex speechlike signals. Peggy B. Nelson and Susan J. Norton (University of Kansas Medical Center, Kansas City, KS 66103)

Speechlike stimuli consisting of /s/-like noise and $F 1$-like frequency sweeps were used for gap detection and discrimination experiments. Five normal-hearing subjects participated in the tasks: (1) detection of gaps in /s/-like noise, (2) discrimination between gaps in noise, (3) detection of gaps between noise and glide, and (4) gap discrimination between noise and glide. Stimuli for the latter two experiments were patterned after synthesized "say" and "stay" speech signals. These stimuli consisted of $120 \mathrm{~ms}$ of high-pass-filter digital white noise (simulating the /s/ phoneme), followed by a silent gap varying from $0-120 \mathrm{~ms}$ in duration, and a 50 -ms linear frequency sweep from $230-611 \mathrm{~Hz}$ ( simulating the $F 1$ transition). The same high-pass-filtered white noise was used for all experiments. An adaptive psychophysical procedure was used in the first two experiments; a method of constant stimuli procedure was used for the complex stimuli tasks because of the shallow-sloping psychometric functions. Subjects required extensive training to reach asymptotic performance levels on the latter two experimental tasks. Results indicated significantly longer durations are required for the complex stimulus tasks.

\section{3:00}

WW8. Gap detection in narrow bands of noise as a function of the number and proximity of the bands. J. H. Grose and J. W. Hall, III (Division of Otolaryngology/Head and Neck Surgery, University of North Carolina at Chapel Hill, Chapel Hill, NC 27599-7070)

The minimum detectable gap in a noise decreases both as the bandwidth of the noise increases and as the number of independent noise bands carrying the gap increases. The present study attempted to relate these two observations by differentiating between the parameters of bandwidth and number of active channels. The stimuli consisted of either one band or five independent bands of noise, each $20 \mathrm{~Hz}$ wide. In all conditions, one of the bands was centered on $1000 \mathrm{~Hz}$. The multiple bands were either juxtaposed to give a single band of noise centered on $1000 \mathrm{~Hz}$ or were spaced in various configurations, resulting in a set of conditions ranging from all bands passing through a single auditory channel to each band passing through an independent channel. The stimuli were presented at a spectrum level equal to that of a $15-\mathrm{dB} \mathrm{SL}, 20-\mathrm{Hz}$-wide band of noise centered on $1000 \mathrm{~Hz}$. The gap was imposed with a 10-ms rise-fall time. Gap thresholds improved with an increased cumulative bandwidth, but more so for the spaced bands than for the juxtaposed bands. [Research supported by AFOSR.]

\section{3:15}

WW9. Effects of a silent temporal interval on discriminability of bursts of reproducible noise. Susan M. Fallon (Department of Psychology, University of Florida, Gainesville, FL 32611) and Donald E. Robinson (Department of Psychology, Indiana University, Bloomington, IN 47405)

A same-different psychophysical method was used to examine the discriminability of bursts of reproducible noise. Bursts in a pair were identical on "same" trials. On "different" trials, bursts were identical except for $\tau$ ms of independent noise located at either the beginning, middle, or end of the burst pairs. Fallon and Robinson [ J. Acoust. Soc. Am. Suppl. 1 78, S46 (1985) ] showed that discriminability depends on the temporal position and proportional duration of the independent noise. The present experiments examined whether a silent interval placed either immediately prior to or following the independent noise improves discriminability. Results showed that a silent interval only improved discriminability if it replaced a portion of the noise common to the pairs of bursts. In this experiment, a brief burst of common noise temporally separated from the independent noise by $90-120$ ms caused a large decrement in performance. These data suggest that interactions occurring after such a long silent interval are unlikely to be generated peripherally. [Work supported by AFOSR.]

WW10. Auditory temporal acuity for dynamic signals. Lawrence L. Feth, Mary E. Neill (Department of Speech and Hearing Science, Ohio State University, Columbus, $O H$ 43210), and Ashok Krishnamurthy (Department of Electrical Engineering, Ohio State University, Colombus, OH 43210)

Previous reports of auditory temporal acuity have generally used static, rather than dynamic, signals; that is, the charactersitics of the signal did not change within its brief duration. Temporal acuity is defined as in Green [Psychol. Rev. 78, 540-551 (1971)]: the shortest time interval within which a listener cannot detect a change in the sound. Auditory temporal acuity is determined using frequency-modulated (FM) sinusoids. The two FM signals used in each block of trial always began at the same initial frequency and moved to the same final frequency. Only the trajectory, the path from initial to final frequency, differed. One trajectory, the glide, moved linearly from initial to final freqency; the other proceeded in a series of discrete steps. As the number of steps increased, the size and duration of an individual step decreased, and the multiplestepped transition more closely approximated the glide. Four well-practiced listeners were asked to identify the interval containing the glide in a 
2-Q, 2AFC task. Frequency transitions of 100-, 200-, and 400-Hz durations of 25,50 , and $100 \mathrm{~ms}$ were centered on octave frequencies from 250 to $4 \mathrm{kHz}$. Signals were presented at $50 \mathrm{~dB}$ SL for each subject. Results for the four listeners were remarkably consistent, with the $75 \%$ discrimination point falling at 7 to $10 \mathrm{~ms}$ for all conditions except for signals centered at $4 \mathrm{kHz}$. Poorer performance at $4 \mathrm{kHz}$ may reflect the loss of synchrony in the frequency region; however, there are some difficulties with that explanation. [Work supported by a grant from AFOSR.]

\section{3:45}

WW11. Discrimination of partially correlated temporal sequences. $R$. D. Sorkin, M. V. Pezzo, and G. C. Elvers (Department of Psychology, University of Florida, Gainesville, FL 32611)

How do observers determine whether two equitone sequences have the same or different temporal patterns? On each trial of this experiment, observers were presented with two sequences of $n$ tones $(n=8,12$, or 16 ; tone duration $=35 \mathrm{~ms}$; frequency $=1000 \mathrm{~Hz}$; level $=71 \mathrm{~dB}$ ). The sequences were played successively and the observers had to report whether the temporal pattern of intertone gaps was the same or different in the two sequences. On half of the trials, the sequences of gaps were identical (perfectly correlated, $\rho=1$ ), and on half of the trials the sequences were partially correlated $(0<\rho<1)$. The gaps were determined by summing two random processes, one common to each sequence and one unique; this procedure allowed control of the correlation, mean, and standard deviation of the gap sequences. Observer performance $\left(d^{\prime}\right)$ increased with the magnitude of the gap variance and decreased with the correlation between the sequences. A model based on computation of the sample correlation, limited by an internal temporal variability of approximately $15 \mathrm{~ms}$, predicted observer performance in a variety of conditions. [Work supported by AFOSR.]

\section{4:00}

WW12. Temporal DLs in isochronic loudness and pitch patterns. Caroline B. Monahan and Ira J. Hirsh (Central Institute for the Deaf, 818 South Euclid, St. Louis, MO 63110)

In an adaptive procedure, listeners discriminated the delay of a single tone in otherwise isochronic six-tone patterns. The temporal DL was measured at three tempos (isochronic intervals of 50,100 , and $200 \mathrm{~ms}$ ) and for four delay positions (tones 2-5). In experiment 1 , patterns comprised loud (forte) and soft (piano) tones: thus FFPPPF, PFPFPF, etc., where $F$ was $80 \mathrm{~dB}$ SPL and $P$ was $70 \mathrm{~dB}$ SPL. In experiment 2 , frequency patterns were "parallels" of the loudness patterns of the first study: thus HHLLLH, LHLHLH, etc., where $\mathrm{H}$ (igh) was $1667 \mathrm{~Hz}$ and L(ow) was $1000 \mathrm{~Hz}$. In both studies, the measure of timing accuracy (Weber ratio $\times 100$ ) was similar to that found in earlier literature for the discrimination of single time intervals, namely, $5 \%-8 \%$ at $200 \mathrm{~ms}, 9 \%-15 \%$ at 100 $\mathrm{ms}$, and $15 \%-25 \%$ at $50 \mathrm{~ms}$. Accuracy was poorest for early serial positions of the delayed tone. This effect occurred primarily at $50 \mathrm{~ms}$ and was significant only in the loudness experiment. In experiment 1 , at 50 and 100 $\mathrm{ms}$, accuracy was about $2 \%$ better when the delayed tone was loud rather than soft. In experiment 2 , accuracy was inversely related to the number of pitch changes per pattern. At $50 \mathrm{~ms}$, accuracy was about $6 \%$ better when the delayed tone and the tone following identical rather than different in frequency. [Work supported by AFOSR.]

FRIDAY AFTERNOON, 1 DECEMBER 1989

ST. LOUIS BALLROOM A, 1:15 TO 3:39 P.M.

\title{
Session XX. Speech Communication X: Acoustics of Speech
}

\author{
Marios Fourakis, Chairman \\ Central Institute for the Deaf, 818 South Euclid Avenue, St. Louis, Missouri 63110
}

Contributed Papers

\section{1:15}

XX1. Some acoustical properties of diphthongs. Michael Gottfried (Central Institute for the Deaf, 818 South Euclid, St. Louis, MO 631 10)

One approach to describing diphthongs is to consider them as consisting of a steady-state nucleus and a glide toward another steady-state segment. This study evaluates this approach when sources of acoustic variation (change of speaker, stress, and rate) are introduced. In a preliminary study, four speakers of Midwestern American English were recorded producing diphthongs in isolation and in the context $/ K \_T /$. On the basis of the first three formants, diphthongs can indeed be characterized in terms of two kinds of spectrally defined states, steady-state and glide. There is, however, variation in the number and order of these states for particular diphthongs. Plots of these spectral patterns in an auditoryperceptual space [J. D. Miller, J. Acoust. Soc. Am. 85, 2114-2134 (1989)] suggested that diphthongs can be differentiated by a combination of distinctive nucleus zones and angular movements wit hin the space. Productions by two speakers of a carrier sentence containing diphthongs under varied stress and rate conditions are being analyzed. Results will be used to evaluate the usefulness of the descriptive scheme noted above and the ability of the auditory-perceptual space to capture differences between diphthongs. Results of this evaluation, in addition to durational measurements of individual diphthongs and their component segments, will be reported. [Work supported by NINCDS.]

XX2. Acoustic characteristics of Xhosa clicks. Bonny E. Sands (Department of Linguistics, UCLA, Los Angeles, CA 90024-1543)

Xhosa, a Bantu language of Southern Africa, has 15 distinct clicks. Clicks are made at three places of articulation-dental, alveo-palatal, and lateral-and with five different accompaniments-voiceless, aspirated, nasalized, murmured, and nasalized murmured. A recording was made of eight speakers saying words containing each of these 15 clicks before each of the five vowels $/ \mathrm{i}, \mathrm{e}, \mathrm{a}, \mathrm{o}, \mathrm{u} /$. The DSP Sona-Graph was used to produce wideband spectrograms, waveforms, and spectra of these utterances. Dental clicks were marked by fricative noise occurring throughout a wide frequency range, and by lack of a clear onset. Alveo-palatal clicks were marked by an abrupt release with little frication. Lateral clicks were more 
affricated than the alveo-palatal clicks. The place of articulation of the click was not observed to have any effect on the formant transitions of the following vowel. Observed vowel effects on clicks were limited to the reduction in amplitude of high-frequency noise before rounded vowels $\mathrm{J} /$ and $/ \mathrm{o} /$. The five accompaniments at each place have a number of phonetic characteristics that will be discussed. [Work supported by NSF.]

\section{1:39}

XX3. Statistical analysis with fundamental frequencies of 12 Korean monophthongs. H. J. Shin and S. W. Yoon ${ }^{a)}$ (Acoustics Research Laboratory, Department of Physics, Sung Kyun Kwan University, Suwon, 440-746, Republic of Korea)

The 12 Korean monophthongs pronounced by five male vocal musicians, each at five fundamental frequencies, are studied by a method of statistical analysis with the fundamental frequencies (SAFF) based on the format analysis. In this study, the SAFF method shows more reasonable results of the format frequencies in the Korean monophthongs. It is also observed that the first and second formants are characterized by the resonance of the cavities of the pharynx and mouth, respectively. The liprounding effect decreases the second-formant frequency. ${ }^{\text {a) }}$ Current address: National Center for Physical Acoustics, University of Mississippi, University, MS 38677.

\section{1:51}

XX4. Effects of consonant context and lexical stress on vowel formant frequencies in continuous speech. Caroline B. Huang (Department of Electrical Engineering and Computer Science and Research Laboratory of Electronics, MIT, Room 36-511, Cambridge, MA 02139)

The effects of consonant context and lexical stress for vowels and vowels in continuous speech have been investigated in previous acoustic studies [e.g., K. Stevens and A. House, J. Speech Hear. Res. 6, 111-128 (1963); P. Delattre, Final Rep., University of California (1969); F. Koopmans-van Beinum, Ph.D. thesis, University of Amsterdam (1980) ]. However, the studies of consonant context and lexical stress involved only isolated words or words in a carrier phrase, and the studies of continuous speech did not consider each consonant context separately. In the present study, formant frequencies are measured in vowels taken from a read story that is well controlled with respect to consonant context and lexical stress. The vowels studied are $/ \mathrm{i}^{\mathrm{y}} /, / \mathrm{e}^{\mathrm{y}} /, / \mathrm{l} /, / \varepsilon /$, and $/ \Lambda /$. The consonant contexts are $/ \mathrm{b} /, / \mathrm{d} /, / \mathrm{g} /, / \mathrm{w} /, / \mathrm{r} /$, and $/ \mathrm{l} /$. The vowels carry primary or secondary lexical stress. The same vowels and contexts are elicited in a retold story and in nonsense words spoken in a carrier phrase. Preliminary results from one speaker indicate that consonant context and lexical stress do affect vowels in continuous speech, as expected. Furthermore, results so far show no gross differences in the effects of these factors on vowels in continuous speech and in the isolated words studied previously. [Work supported in part by NIH.]

\section{2:03}

XX5. Higher harmonics and formants analysis of speech signals. Victor F. Coronel and Sultan Catto (Department of Natural Sciences, Baruch College, 17 Lexington Avenue, New York, NY 10010)

Spectral analysis of windowed segments (12.8-52.8 ms in duration), of the approximately steady portion of vowel sounds, in the context CVC, was analyzed for several individuals at various SPL levels for each subject. It was observed that the signals with larger SPL values were much richer in harmonics and formants. A relation between the straining of the vocal cords and the higher harmonics is established and its relation with nonlinear models of the vocal chords and vocal tract examined. The higher harmonics of the fundamental frequency, sixth and above, and the higher- order formants, third and above, when present for certain vowel sounds, may be of particular importance as diagnostic tools in vocal-fold-vocaltract clinical examination.

\section{2:15}

XX6. Automatic correction of formant tracks: A progress report. J. Andrew Fingerhut and James D. Miller (Central Institute for the Deaf, 818 South Euclid, St. Louis, MO 63110)

Since commercially available formant trackers are often inconsistent, much hand-editing is required to correct their outputs. A software package is currently being developed that may reduce this problem. Two female and two male speakers were recorded producing ten vowels in Svowel- $T$ context, each vowel twice. Boundaries between burst-friction, glottal-source, and silent segments are located using zero crossing and rms energy measurements. Pitch and formant-frequency values are extracted using the API and SGM commands of ILS. Our software corrects the pitch contour and calculates a sensory reference from it [J. D. Miller, J. Acoust. Soc. Am. 85, 2114-2134 (1989) ]. Rules based on the relation between peak frequencies and this sensory reference are utilized to label the peaks as formants and the resulting formant tracks are then low-pass filtered. In this way, satisfactory tracking is obtained for all 80 syllables. [Work supported by AFOSR and NIDCD.]

\section{2:27}

XX7. Specification of vowel height using $F 1$ trajectories. H.S. Gopal and Joyce Manzella (Department of Speech and Hearing Sciences, University of California at Santa Barbara, Santa Barbara, CA 93106)

Recently, the claim has been made that both acoustic and perceptual ambiguities between high and low vowels can be reduced when $F 1$ time/ frequency variations, rather than $F 1$ "target" values, are considered [Di Beneditto, J. Acoust. Soc. Am. 86, 55-66 (1989)]. The current study investigates the efficacy of the $F 1$ time/frequency variation (the $F 1$ trajectory) as an acoustic measure of vowel height using an extended set of vowels. Additionally, the effect of speaking rate on these trajectories as it relates to vowel height is investigated. Four high-low vowel pairs in $/ \mathrm{pVC} /$ target syllables containing voiced and voiceless stops and fricatives are included. These syllables were produced at various rates in sentence context by four American English speakers, two males and two females. $F 1$ trajectory versus $F 1$ maximum measures are discussed in terms of their relative efficacy in classifying vowels into high and low groups.

XX8. Acoustic comparison of unvoiced fricatives in telephone speech versus typical speech. David W. Petr, Todd G. Ellenberger, and Victor S. Frost (Telecommunications and Information Sciences Laboratory, University of Kansas, Lawrence, KS 66045)

The results of a pilot study designed to compare the acoustic properties of unvoiced fricatives in telephone speech versus speech typically used for acoustic-phonetic analyses are reported. Two new characterizing parameters for the unvoiced fricatives are introduced: the half-power bandwidth (resonance width) and a three-point energy versus time contour. It is found that the average fricative power normalized by the average adjacent vowel power is considerably larger in the telephone speech for all speakers, fricatives, and fricative positions studied. Also, half-power bandwidths tend to be larger for [f] and $[\theta]$ in telephone speech, but smaller for $[s]$ and $\left[\int\right]$. The study yields a preliminary understanding of these acoustic differences in terms of the differences in vocal effort, microphone placement, and frequency response and bandwidth of the recording equipment. Finally, possible distinguishing perceptual cues for the different recording environments in terms of the acoustic differences are hy- 
pothesized. In particular, the energy-time contour of $[\theta]$ exhibits a peculiar high-low-high pattern that is most pronounced in the telephone recordings. [ Work supported by AT\&T, NSF, and the University of Kansas.]

2:51

XX9. Perceptual judgments of modified deaf speech. Grace H. YeniKomshian (Department of Hearing and Speech Sciences, University of Maryland, College Park, MD 20742), H. Timothy Bunnell (A. I. Dupont Institute, Wilmington, DE 19899), and James J. Mahshie (Gallaudet University, Washington, DC 20002)

The perceptual effects of modifying speech produced by deaf talkers were investigated to examine, in part, the changes necessary for disordered speech to be judged normal. Recordings of the rainbow passage produced by a number of deaf male speakers were studied and three passages judged to be moderately disordered were selected. The word "beautiful" was extracted from these passages and from a similar passage recorded by a hearing talker. Each of the deaf talkers' productions was paired with the normal production to make two types of continua. One type was based on a time warping of the disordered speech to fit the temporal structure of the normal speech, while the other was based on a time warping of the normal speech to the temporal structure of the disordered speech. Each continuum contained ten steps, ranging from disordered to normal spectral properties. Graduate students in the fields of speech and hearing judged whether the stimuli sounded normal or disordered. Continua based on the normal talker's timing provided ordered responses showing an increasing proportion of "normal" responses for steps approaching normal spectra. For continua based on the deaf talkers' timing, however, responses were generally not ordered across the spectral continuum. The relative merits of temporal and spectral modifications will be discussed.

\section{3:03}

XX10. Power spectra of some modes of speech. Igor V. Nábělek (Department of Audiology and Speech Pathology, College of Liberal Arts, University of Tennessee, Knoxville, TN 37996-0740)

The data to be reported represent an extension of the previous study "A comparison of spectra of loud and whispered speech" [Nábĕlek and Maroonroge, J. Acoust. Soc. Am. Suppl. 1 75, S83 (1984) ]. Power spectra were determined for three modes of running speech: phonated conversational speech, phonated monotonous speech, and whispered speech. The average spectrum of the phonated conversational speech had a broad maximum around $300 \mathrm{~Hz}$; the spectra of the phonated monotonous speech had pronounced peaks at the fundamental frequency and its harmonics. The average spectrum of the whispered speech was rather flat at low frequencies, and it was similar to the spectrum of the phonated speech at frequencies above $2000 \mathrm{~Hz}$. The implications of differences bet ween the spectra for testing will be discussed.

XX11. Accurate measurements of jitter, shimmer, and glottal turbulence noise from sustained vowels. Hideki Kasuya, Yoshinobu Kikuchi, Zaoming Huang, Osamu Komuro, and Keisuke Maeda (Faculty of Engineering, Utsunomiya University, Utsunomiya 32 I, Japan)

There has been much research on the measurement of jitter, shimmer, and glottal turbulence noise from sustained vowels, primarily aiming at acoustic quantification of pathological voices. Many problems remain unsolved, however, specifically those of accurate methods for the pitch extraction and noise measurement and of quantification of shimmer. This paper describes a sophisticated method for the pitch period determination based on a cycle-to-cycle similarity of the waveform and a novel method to quantify shimmer characteristics. In this method, shimmer is analyzed for the individual harmonic component of the waveform in terms of its variations in amplitude and phase characteristics. Acoustic measurements of glottal turbulence noise due to insufficient vocal-fold closure are also discussed. Efficacy of the new methods for the measurement of jitter, shimmer, and noise is compared with previous work using pathological voice samples. [Work supported by a Grant-in-Aid from the Ministry of Education, Science and Culture, Japan.]

\section{3:27}

XX12. Sound transmission to the chest wall in the subglottal respiratory system at low frequencies. George R. Wodicka (School of Electrical Engineering, Purdue University, West Lafayette, IN 47907)

The amplitude of sound transmission from the mouth to sites overlying the extrathoracic trachea and on the posterior chest wall was measured from 100 to $600 \mathrm{~Hz}$. Bandlimited white noise was input into the mouth of each subject with the glottis open at resting lung volume, and measurements were performed using accelerometers. The ratios of the transfer functions between the tracheal site and the source, and the chest wall site and the source, were estimated. All of the resulting spectra exhibited a peak that occurred for the group at $129 \pm 6 \mathrm{~Hz}$ with a $Q$ of $2.2 \pm 0.4$. Also, a trend of decreasing spectral energy as frequency increased from the peak location was observed $(-17, \pm 5 \mathrm{~dB} / \mathrm{oct})$. The fundamental radial mode of a model thoracic cavity, which is a large rigid cylinder filled uniformly with lossless lung tissue, provides a good estimate of this low-frequency resonance. This agreement suggests that thoracic cavity resonances may have particularly important effects on sound transmission in the subglottal respiratory system at frequencies below roughly $250 \mathrm{~Hz}$. 